\title{
EXPONENTIAL DECAY OF CORRELATIONS FOR FINITE HORIZON SINAI BILLIARD FLOWS
}

\author{
VIVIANE BALADI, MARK F. DEMERS, AND CARLANGELO LIVERANI
}

In memoriam Nikolai Chernov 1956-2014.

\begin{abstract}
We prove exponential decay of correlations for the billiard flow associated with a two-dimensional finite horizon Lorentz Gas (i.e., the Sinai billiard flow with finite horizon). Along the way, we describe the spectrum of the generator of the corresponding semi-group $\mathcal{L}_{t}$ of transfer operators, i.e., the resonances of the Sinai billiard flow, on a suitable Banach space of anisotropic distributions.
\end{abstract}

\section{Contents}

1. Introduction and statement of results

1.1. The main results (Theorems 1.2 and 1.4, Corollary 1.3) 4

2. Definition of the norms

2.1. Stable and unstable cones for the flow

2.2. Admissible stable and unstable curves for the flow

2.3. The norms $|\cdot|_{w},\|\cdot\|_{s},\|\cdot\|_{u},\|\cdot\|_{0}$, and the spaces $\mathcal{B}$ and $\mathcal{B}_{w}$

3. Preliminary lemmas

3.1. Growth and distortion

3.2. Embeddings of smooth functions in $\mathcal{B}$. Compactness of $\mathcal{B}$ in $\mathcal{B}_{w}$

4. Lasota-Yorke-type bounds and strong continuity for the semi-group $\mathcal{L}_{t} \quad 26$

4.1. Weak stable, strong stable, and neutral norm estimates for $\mathcal{L}_{t} \quad 27$

4.2. Unstable norm estimate for $\mathcal{L}_{t}$

4.3. Strong continuity of $\mathcal{L}_{t}$ on $\mathcal{B}$

5. Quasi-compactness of the resolvent $\mathcal{R}(z)$, first results on the spectrum of $X$

5.1. Norm estimates for $\mathcal{R}(z)$

6. Construction of approximate unstable Lipschitz foliations 41

6.1. Constructing the regular part of the map-foliation, (iii-iv) 43

6.2. Smoothness of the regular part of the map-foliation, (v-vi-vii) 445

6.3. Interpolating the map-foliation across the gaps, (vi) 49

6.4. Checking (vii) for the map-foliation interpolated across the gaps 53

6.5. Lifting the map-foliation to a flow foliation, checking (i-viii)

7. Mollification operators and embeddings $\quad 57$

7.1. Mollification operators $\quad 60$

Date: June 6, 2017, amended following referees' reports.

CL acknowledges the partial support of the European Advanced Grant Macroscopic Laws and Dynamical Systems (MALADY) (ERC AdG 246953). MD was partially supported by NSF grants DMS 1101572 and 1362420. Part of this work was carried out by VB and MD at the Newton Institute in 2013 (Mathematics for the Fluid Earth), during visits of VB and MD to Roma in 2013, 2014, 2015, and a visit of MD to Paris in 2014, using the Action incitative ENS Spectres en dynamique, géométrie et théorie des nombres. VB is grateful to Peter Bálint for his patient explanations on billiards and thanks Sébastien Gouëzel for many key ideas. We thank I.P. Toth for several useful remarks. We are grateful to the two anonymous referees for many constructive comments. 
7.2. Proofs of Lemmas $7.3-7.5$

8. The Dolgopyat cancellation estimate (Lemma 8.1) 67

9. Completing the proofs (exponential mixing of $\Phi_{t}$ and resonances of $X$ )

9.1. Proofs of Theorem 1.2 and $1.4 \quad 79$

9.2. Proof of Proposition $9.1 \quad 80$

Appendix A. Proofs of Lemmas 6.6 and 6.7 (approximate foliation holonomy) 83

Appendix B. Estimates for the Dolgopyat bound (Lemmas 8.7, 8.8, and 8.9)

References $\quad 92$

\section{InTRODUCTION AND STATEMENT OF RESUlts}

This paper completes, on a conceptual level, the study of decay of correlations of planar dispersing billiard systems initiated by Sinai's seminal papers [S1, S2] in which he extended the ideas of Hopf and Anosov to the case of piecewise smooth dynamical systems. Sinai's breakthrough prompted several works establishing ergodicity for more and more general systems. Yet, the quantitative study of their mixing properties had to wait almost twenty years until [BSC] established sub-exponential decay of correlations (and the Central Limit Theorem) for the collision map associated to certain dispersing billiard systems. The question remained if the discontinuities prevented exponential decay of correlations or not. The question was settled in [L1, Y, [C1] where exponential decay of correlations was established for a large class of discrete-time dynamical systems with discontinuities (including Poincaré maps for the finite horizon Lorentz gas in [Y], and more general dispersing billiards in [C1]).

These results for the billiard collision map did not settle the question of the rate of decay of correlations for the billiard flow. It is well known that this seemingly uneventful step is highly non trivial: In the case of smooth systems it took 26 years to go from the proof of exponential decay of correlations for Anosov maps [S3, $\mathrm{R}$ ] to the first results, by Dolgopyat, on exponential decay of correlations for Anosov flows [Do]. The first progress for dispersing billiard flows was made by Melbourne [M] and Chernov [C2], who proved super-polynomial and stretched-exponential decay, respectively. The methods of [Y, $\mathrm{M}$, , C2 ] employ some kind of countable Markov partitions. As pointed out by one of the referees, two very recent works have successfully combined Dolgopyat arguments with countable Markov partitions (in the spirit of [BV, AGY]) to get exponential decorrelations for (non-billiard) hyperbolic flows with singularities: K. Burns et al. [BMMW] for some Weil-Petersson flows and Araújo-Melbourne [AM] for the Lorenz attractor. However, these works do not constitute compelling evidence for dispersing billiard flows, the difficulties of which come from the severe lack of smoothness of the foliations (which are measurable, but not continuous). Indeed, in AM the stable foliation is Hölder and consists of long leaves. The situation of [BMMW] is even simpler, since a very good description of the structure of the singularities is available, and the foliation is rather regular. It seems much more difficult (at least to the authors of the present paper) to implement Dolgopyat-type arguments with countable Markov partitions to get exponential mixing for dispersive billiard flows. It is thus natural to try a more direct line of attack, studying the transfer operators on suitable spaces 11 anisotropic distributions defined on the manifold, thus bypassing non-intrinsic constructions.

The first exponential decay result for piecewise smooth hyperbolic flows was obtained by BL] who used such a direct functional approach: They were able to build on Liverani's version [L2] of the Dolgopyat argument for contact Anosov flows and an anisotropic space construction from

\footnotetext{
${ }^{1}$ Unfortunately, the spaces introduced e.g. in BT1, GL, FRS, GLP for Anosov diffeomorphisms or flows do not work well in the presence of discontinuities.
} 
[BG] to prove exponential decay for a large class of piecewise smooth contact hyperbolic flows. However, this class did not contain billiards, since the blowup of derivatives at the boundary of the domain (corresponding to grazing orbits in billiards) was not allowed. In parallel, [DZ1] succeeded in adapting the anisotropic space previously introduced for piecewise hyperbolic discrete-time systems by [DL] to allow for the blowup of derivatives at boundaries, giving a new functional proof of Young's [Y] exponential mixing for the billiard map. (This function space approach has recently been applied to a wide variety of billiard maps and their perturbations [DZ2, DZ3].)

In the present paper, we exploit the fact that the billiard flow preserves a contact form and combine the methods of [BL] with the function spaces of [DZ1] to establish exponential decay of correlations (Corollary 1.3) for the billiard flow associated with a finite horizon Lorentz gas. As a byproduct of our proof, we obtain information on the resonances of the billiard flow (Theorem 1.4), that is, the spectrum of the generator $X$ of the semi-group $\mathcal{L}_{t}$ given by the flow. We warn the reader that, just like in [L2, BL, although we do obtain a spectral gap for the spectrum of the generator $X$ on an anisotropic Banach space $\mathcal{B}$, our method does not show that the time-one transfer operator $\mathcal{L}_{1}$ has a spectral gap on $\mathcal{B}$. (Note that $X$ is closed but not bounded on $\mathcal{B}$.) The spectral gap of $\mathcal{L}_{1}$ on a (different) anisotropic Banach space was obtained by Tsujii ([Ts, Thm 1.1]) in the easier case of smooth Anosov flows.

One key technical hurdle we had to overcome, in order to carry out the Dolgopyat cancellation argument from [L2, BL] in the dispersive billiard setting, is the construction in Section 6 of a suitable approximate (fake) unstable foliation for the billiard, see Theorem 6.2 for a detailed description of its properties. This (a posteriori very natural) delicate construction is one of the main novelties of the present work (see also Remark1.1 below). Along the way we discovered that using in addition the analogous fake stable foliation allows a much more systematic writing of the Dolgopyat cancellation argument (Section [8). Note also that we replace the $\mathcal{C}^{1+\varpi}$ estimates on the holonomies used in [BL] by "four-point conditions" (see condition (vii) and Lemma 6.6 in

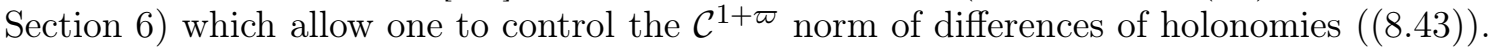

Remark 1.1. The construction of Section [6 and in particular properties (i)-(viii) there, can be easily used to prove the following interesting property of the stable and unstable foliations of the billiard flow (which are only measurable): For each small enough $\eta>0$, there exists a set of Lebesgue measure at most $C \eta^{\frac{4}{5}}$, the complement of which is foliated by leaves of the stable (or unstable) foliation with length at least $\eta$ such that the foliation is Whitney-Lipschitz, with Lipschitz constant not larger than $\mathrm{C \eta}^{-\frac{4}{5}}$. (See Remark 5.3 on the improvement of this exponent.) An analogous statement holds for the billiard map. To our knowledge, this fact was not previously known. We refer to [KS] for related information on dynamical foliations of billiards.

The present work finally settles the issue of whether billiard flows can have exponential decay of correlations. In addition, we obtain new spectral information and we develop new tools. Many problems remain open (more general dispersing billiards, higher dimensional billiards, dynamical zeta functions - which would give another interpretation of the resonances - other Gibbs states etc.), and we hope that some of the techniques in the present paper may be useful towards their solution. Some limit theorems on the billiard flow can be obtained from information on the Poincaré map (see [MT] for the central limit theorem 3 and almost sure invariance principle, see $[\mathrm{MN}]$ for large deviations, see [Pe] for some Berry-Esseen bounds). Although the existing proofs [I] of local limit theorems with error terms are based on a spectral gap for the transfer operator $\mathcal{L}_{1}$, we hope that a spectral gap for (suitable perturbations of) the generator $X$ of the flow will

\footnotetext{
${ }^{2}$ It suffices to take $\eta<L_{0}^{5}$, where $L_{0}$ is from Lemma 3.8

3 Speed estimates can be found in [LP].
} 
lead to e.g., a local limit theorem with error terms, better Berry-Esseen estimates, and rate functions for large deviations.

We remark that the results of the present paper do not apply to the billiard flow corresponding to an infinite horizon periodic Lorentz gas. Recall that although the discrete time collision map for such billiards enjoys exponential decay of correlations [C1], it is anticipated by physicists [FM, IMM] that correlations for the flow decay at a polynomial rate.

The paper is as self-contained as possible, with precise references to the book by Chernov and Markarian [CM] or to the previous works [BL] or [DZ1], whenever we use non trivial facts. Regarding operator-theoretical or functional-analytic background, very little is expected from the reader, and we again give precise references to Davies [Da].

1.1. The main results (Theorems $\mathbf{1 . 2}$ and 1.4 , Corollary 1.3 ). We shall state our results in the setting of the Sinai billiard flow. A more general axiomatic setting can probably be considered in the spirit of [DZ3].

Let $B_{i}, i=1, \ldots d$, denote open, convex sets in the two-torus $\mathbb{T}^{2}=\mathbb{R}^{2} / \mathbb{Z}^{2}$. We assume that the closures of the sets $B_{i}$ are pairwise disjoint and that their boundaries $\Gamma_{i}$ are $\mathcal{C}^{3}$ curves with strictly positive curvature. We consider the motion of a point particle in the domain $Q:=\mathbb{T}^{2} \backslash\left(\cup_{i=1}^{d} B_{i}\right)$ undergoing elastic collisions at the boundaries and maintaining constant velocity between collisions. At collisions, the velocity vector changes direction, but not magnitude. Thus we set the magnitude of the velocity vector equal to one and view the phase space of the flow as three-dimensional.

We adopt the coordinates $Z=(x, y, \omega)$ for the flow, where $(x, y) \in Q$ is the particle's position on the table, and $\omega \in \mathbb{S}^{1}$ (we view $\mathbb{S}^{1}$ as the quotient of $[0,2 \pi]$, identifying endpoints) is the angle made by the velocity vector with the positive $x$-axis. The quotient

$$
\Omega:=\left\{(x, y, \omega) \mid(x, y) \in Q, \quad \omega \in \mathbb{S}^{1}\right\} / \sim
$$

is often used as the phase space for the billiard flow $\Phi_{t}: \Omega \rightarrow \Omega$, where $\sim$ identifies ingoing and outgoing velocity vectors at the collisions, via the reflection with respect to the boundary of the scatterer at $Z \in \partial Q$. (It is then customary to work with the outgoing - post-collisional velocity vector at collisions.) The topological metric space $\Omega$ can be endowed with Hölder (non differentiable) charts. It is thus more convenient to work with the $\mathcal{C}^{2}$ manifold with boundary

$$
\Omega_{0}:=\left\{(x, y, \omega) \mid(x, y) \in Q, \quad \omega \in \mathbb{S}^{1}\right\} \subset \mathbb{T}^{3}=\mathbb{T}^{2} \times \mathbb{S}^{1} .
$$

For nonzero $t$, we may consider the time-t billiard flow $\Phi_{t}$ on $\Omega_{0}$. The time-zero map $\Phi_{0}$ is the identity in the interior of $\Omega_{0}$, and also at grazing collisions in $\partial \Omega_{0}$, where the incoming and outgoing angles coincide. (Note that at $(x, y) \in \partial Q$ there are exactly two grazing angles, $\omega_{g r}(x, y) \in[0, \pi)$ and $\omega_{g r}(x, y)+\pi$. These two angles divide the circle in the two arcs of ingoing, respectively outgoing, angles.). For $(x, y) \in \partial Q$ and $\omega^{-}$a non-grazing incoming angle, we have that the (reflected) outgoing angle $\omega^{+}$(i.e., so that $\Phi_{0}\left(x, y, \omega^{-}\right)=\left(x, y, \omega^{+}\right)$) is different from $\omega^{-}$. In particular, $\Phi_{t}\left(x, y, \omega^{-}\right)=\Phi_{t}\left(x, y, \omega^{+}\right)$for such $\left(x, y, \omega^{-}\right)$and all $t>0$, so that the time- $t$ map is not injective. Similar properties hold for $\omega$ a non-grazing outgoing angle, reversing time. The nature of the Banach space norms defined below will ensure that these apparent flaws in the flow do not create problems 5

The billiard flow is our primary object of study, but it will sometimes be convenient to use the billiard map (also called collision map), i.e., the Poincaré map $T: \mathcal{M} \rightarrow \mathcal{M}$ of the flow on the union of scatterers $\mathcal{M}=\cup_{i=1}^{d} \Gamma_{i} \times\left[-\frac{\pi}{2}, \frac{\pi}{2}\right]$. Natural coordinates for the collision map $T$

\footnotetext{
${ }^{4}$ In view of defining $\mathcal{C}^{2}$ stable curves which can touch the scatterers on their endpoints, $\mathcal{C}^{2}$ test functions (when considering $\mathcal{C}^{\ell}$ functions on $\Omega_{0}$, we mean differentiable in the sense of Whitney, viewing $\Omega_{0}$ as a subset of the three-torus).

${ }^{5}$ In particular strong continuity holds for the transfer operator $\mathcal{L}_{t}$ associated to $\Phi_{t}$ for $t>0$, see Lemma 4.6
} 
are $(r, \varphi)$, where $r$ represents the position on the boundary of the scatterer, parametrized by arclength and oriented positively with respect to the domain $Q$, and $\varphi$ is the angle made by the outgoing (post-collision) velocity vector with the outward pointing normal to the boundary of the scatterer. Let $\tau(Z)$ denote the time when the particle at $Z \in \Omega_{0}$ first collides with one of the scatterers. (It is known that $\tau$ is a piecewise $1 / 2$ Hölder function, see the proof of Lemma 3.4.) We assume that the table has finite horizon, i.e., there exists $\tau_{\max }<\infty$ such that $\tau(Z) \leq \tau_{\max }$ for all $Z \in \Omega_{0}$. Since we have assumed the scatterers are a positive distance apart, there exists a constant $\tau_{\min }>0$ such that $\tau(r, \varphi) \geq \tau_{\min }$ for all $(r, \varphi) \in \mathcal{M}$.

The billiard flow preserves the normalised Lebesgue measure (see e.g. [CM, Def. 2.23])

$$
m=(2 \pi|Q|)^{-1} d x d y d \omega,
$$

where $|Q|$ denotes the area of the billiard domain. Our first result gives a precise description of the Sinai billiard flow decorrelations:

Theorem 1.2 (Fine correlation structure for the Sinai billiard flow). Let $\Phi_{t}: \Omega_{0} \rightarrow \Omega_{0}$ be a finite horizon (two-dimensional) billiard flow associated to finitely many scatterers $B_{i}$ with $\mathcal{C}^{3}$ boundaries of positive curvature and so that the $\bar{B}_{i}$ are pairwise disjoint. Then there exist a constant $v_{D o}>0$, and for any $v_{1}<v_{D o}$ a constant $C_{1}>0$, a finite dimensional vector space $F \subset\left(\mathcal{C}^{1}\left(\Omega_{0}\right)\right)^{*}$, a (nontrivial) bounded operator $\Pi: \mathcal{C}^{2}\left(\Omega_{0}\right) \cap \mathcal{C}^{0}(\Omega) \rightarrow F$ and a matrix $\widehat{X} \in L(F, F)$ so that for any $\psi \in \mathcal{C}^{1}\left(\Omega_{0}\right)$ and $f \in \mathcal{C}^{2}\left(\Omega_{0}\right) \cap \mathcal{C}^{0}(\Omega)$ we have

$$
\left|\int\left(\psi \circ \Phi_{t}\right) f d m-\int \psi e^{\widehat{X} t} \Pi f d m\right| \leq C_{1}|f|_{\mathcal{C}^{2}\left(\Omega_{0}\right)}|\psi|_{\mathcal{C}^{1}\left(\Omega_{0}\right)} \cdot e^{-v_{1} t}, \forall t \geq 0 .
$$

In addition, the spectrum $\operatorname{sp}(\widehat{X}) \subset\left\{z \in \mathbb{C}:-v_{\text {Do }}<\Re(z)<0\right\} \cup\{0\}$, and zero is a simple eigenvalue with contribution to $\Pi(f)$ given by $\int f d m$.

A lower bound for $v_{D o}$, given by Proposition 9.1 below, can be explicitly traced from the proof of that proposition (it depends on the constants $\gamma_{D o}$ and $C_{D o}$ from Lemma 8.1 we show there that $\gamma_{D o} \geq \frac{1}{1222}$ and $C_{D o}$ can also be made explicit, depending in particular on the hyperbolicity exponents (1.2), if desired) but is rather small and not optimal. We refrain from stating the lower bound for $v_{D o}$ which would be very unwieldy.

Given Theorem 1.2 it is immediate, by a standard approximation argument, to obtain our main result of exponential decay of correlations for the Lebesgue invariant measure of the billiard flow (1.1) and Hölder test functions.

Corollary 1.3 (Exponential mixing of the Sinai billiard flow). Under the hypotheses of Theorem 1.2, for any $\kappa \in(0,1)$ there exists $0<v_{\text {corr }}(\kappa)<v_{\text {Do }}$ and $C_{\text {corr }}(\kappa)>0$ so that for any $\psi, f \in \mathcal{C}^{\kappa}\left(\Omega_{0}\right)$ we have

$$
\left|\int\left(\psi \circ \Phi_{t}\right) f d m-\int \psi d m \cdot \int f d m\right| \leq C_{c o r r}(\kappa)|\psi|_{\mathcal{C}^{\kappa}\left(\Omega_{0}\right)}|f|_{\mathcal{C}^{\kappa}\left(\Omega_{0}\right)} \cdot e^{-v_{c o r r}(\kappa) t}, \forall t \geq 0 .
$$

In Corollary 1.3, we may take $\psi$ or $f$ (or both) to be the velocity function (which belongs to $C^{\kappa}\left(\Omega_{0}\right)$ ), or the free flight function $\tau$ (the piecewise Hölder function $\tau$ can be approached by a function in $C^{2}\left(\Omega_{0}\right)$, either by adapting the proof of [DZ1, Lemma 3.7] or by noticing that $\tau$ belongs to a fractional Sobolev space $H_{p}^{\kappa}$ with $\kappa>0$ and $p>2$, and using mollification.).

\footnotetext{
${ }^{6}$ For integer $\ell$, we set $|f|_{\mathcal{C}^{\ell}\left(\Omega_{0}\right)}=\sum_{k=0}^{\ell} \sup _{x \in \Omega_{0}} \max _{|\vec{k}|=k}\left|\partial^{\vec{k}} f(x)\right|$, where $\partial^{\vec{k}}$ is the partial derivative associated to $\vec{k}=\left(k_{1}, k_{2}, k_{3}\right) \in \mathbb{Z}_{+}^{3}$ and $|\vec{k}|=k_{1}+k_{2}+k_{3}$, using the natural chart on the torus.

7 If $\kappa \in(0,1)$, we set $|f|_{\mathcal{C}^{\kappa}\left(\Omega_{0}\right)}=\sup _{x \in \Omega_{0}}|f(x)|+\sup _{x \neq y \in \Omega_{0}} \frac{|f(x)-f(y)|}{d(x, y)}$, for the distance on $\Omega_{0}$ induced by $\mathbb{T}^{3}$.
} 
The proof of Theorem 1.2 will be completed in Subsection 9.1. It is based on a study of the semi-group of transfer operators defined by (a priori just measurably, since $\Phi_{t}$ is not injective)

$$
\mathcal{L}_{t} f=f \circ \Phi_{-t}, \quad t \geq 0,
$$

acting on a suitable Banach space $\mathcal{B}$ (see Definition 2.12) of anisotropic distributions. Along the way, we shall obtain information on the "resonances" of the flow, that is the spectrum (on $\mathcal{B}$ ) of the generator $X$ of the semi-group, defined by $X f=\lim _{t \downarrow 0} \frac{\mathcal{L}_{t} f-f}{t}$, whenever the limit exists (see Section (5). To state the corresponding result, letting $\mathcal{K}(r)$ denote the curvature at a point $r$ on the boundary $\Gamma_{i}$ of a scatterer, first note that our assumptions ensure that there exist constants $0<\mathcal{K}_{\min } \leq \mathcal{K}_{\max }<\infty$ with $\mathcal{K}_{\min } \leq \mathcal{K}(r) \leq \mathcal{K}_{\max }$, for all $r \in \Gamma_{i}$. Then set

$$
\Lambda_{0}=1+2 \mathcal{K}_{\min } \tau_{\min }, \quad \Lambda=\Lambda_{0}^{1 / \tau_{\max }} .
$$

$\Lambda_{0}$ and $\Lambda$ are the hyperbolicity exponents (minimum expansion and maximum contraction) of the billiard map and flow, respectively (see [CM, eqs. (4.6), (4.17), (4.19)] for $\Lambda_{0}$ ). Our value for $v_{D o}$ satisfies $v_{D o}<\frac{1}{6} \log \Lambda$.

Theorem 1.4 (Resonances of the Sinai billiard flow). For any $v_{\text {ess }} \in\left(v_{D o}, \frac{1}{4} \log \Lambda\right)$ there exists a Banach space $\mathcal{B}$ of distributions on $\Omega_{0}$ (with the embeddings $\mathcal{C}^{1}\left(\Omega_{0}\right) \hookrightarrow \mathcal{B} \hookrightarrow\left(\mathcal{C}^{1}\left(\Omega_{0}\right)\right)^{*}$ ) so that $X$ is a closed operator on $\mathcal{B}$ with a dense domain, and the spectrum $\operatorname{sp}(X)$ on $\mathcal{B}$ satisfies:

a) The intersection $\operatorname{sp}(X) \cap\left\{\Re(z)>-v_{\text {ess }}\right\}$ consists of (at most countably many) isolated eigenvalues $\Sigma=\left\{z_{j}, j \geq 0\right\}$ of finite multiplicities. ("Discrete eigenvalues of the generator.")

b) There exists $0<v_{0} \leq v_{D o}<v_{\text {ess }}$ so that $\operatorname{sp}(X) \cap\left\{\Re(z)>-v_{0}\right\}=\left\{z_{0}=0\right\}$, which is an eigenvalue of algebraic multiplicity equal to one. ("Spectral gap.")

c) $\operatorname{sp}(X) \cap\left\{z \in \mathbb{C}: \Re(z)>-v_{D o}\right\}=s p(\widehat{X})$, where multiplicities coincide. ("Resonances.")

(Note that (c) implies (b), taking $v_{0}=v_{D o}$, but (c) does not imply (a), since $v_{\text {ess }}$ can be taken arbitrarily close to $\frac{1}{4} \log \Lambda$. Note also that Theorem 1.4 does not imply Theorem 1.2 immediately — we use $\mathrm{Bu}$ - since (b) gives a spectral gap for $X$ and not for any individual $\mathcal{L}_{t}$.) Claims (a) and (b) encapsulate Lemma 7.5. Corollaries 5.4 and 9.2, together with Remark 5.3. It would of course be very interesting to have examples where $\operatorname{sp}(\widehat{X}) \neq\{0\}$ and examples where $\Sigma \backslash \operatorname{sp}(\widehat{X})$ is not empty (even better, an infinite set).

Remark 1.5 (On hyperbolicity, transversality, and complexity). Billiards are well known to possess families of invariant stable and unstable cones, and this is of course crucial to get exponential mixing. For abstract piecewise smooth and hyperbolic (or expanding) systems in dimension two or higher, it is also essential in order to get a spectral gap that "hyperbolicity dominates complexity," and a corresponding complexity assumption is present e.g. in $[\mathrm{Y}, \mathrm{BL}$. This assumption is well known to hold for billiards and is encapsulated (and precisely quantified) in the one-step expansion (see e.g. [CM, Lemma 5.56]) used by Demers-Zhang [DZ1] for the discrete time billiard, and that we use several times below (most importantly in Lemma 3.8). An additional essential ingredient in the piecewise hyperbolic case is transversality between the stable (or unstable) cones and the hyperplane 8 of discontinuities or singularities. In the context of Sinai billiard maps, uniform transversality between the stable cones and the boundaries of all homogeneity layers (a sequence of hyperplanes approaching the hyperplane of grazing singularities, see Definition (2.5) is again a well known property. For the flow, the transversality is more delicate, and in fact we make use of a weak transversality property throughout (as a single example, we mention Lemma 8.2), yet it is neither easy nor necessary to isolate a specific statement or definition embodying the notion of weak transversality.

\footnotetext{
${ }^{8}$ In the language of $[\mathrm{BL}]$ the transversality condition is relative to the lateral sides of the flow boxes.
} 
The paper is organised as follows: Section 2 contains definitions of our norms (based on a notion of admissible stable curves $W \in \mathcal{W}^{s}$, see Definition 2.7) and Banach spaces $\mathcal{B}$ and $\mathcal{B}_{w}$ of distributions on $\Omega_{0}$ (Definition 2.12). In Section 3, we prove on the one hand some lemmas on growth and distortion under the action of the flow $\Phi_{t}$, in particular giving invariance of the class $\mathcal{W}^{s}$ of stable curves (modulo the necessary "cutting up" causing the complexity evoked in Remark 1.5, and which is controlled by Lemma 3.8), and on the other hand the key compact embedding statement $\mathcal{B} \subset \mathcal{B}_{w}$ (Lemma [3.10). Just like in [L2, $\left.\mathrm{BL}\right]$, our spectral study of the generator $X$ is based on an analysis of the resolvent

$$
\mathcal{R}(z)=(z \mathbf{I} \mathbf{d}-X)^{-1} \quad z=a+i b \in \mathbb{C} .
$$

In Section 5, we obtain Lasota-Yorke type estimates (Proposition 5.1 and its consequences Corollaries 5.2 and 5.4) on $\mathcal{R}(z)$ which give (a little more than) claim (a) of Theorem 1.4. Since the resolvent $\mathcal{R}(z)$ can be expressed as the Laplace transform (5.1) of the transfer operator $\mathcal{L}_{t}$, these Lasota-Yorke estimates follow from estimates on the transfer operator proved in Section 4. The approximate unstable foliation is constructed and studied in Section [6 and Appendix A. The delicate Dolgopyat-type cancellation Lemma 8.1 which bounds

$$
\int_{W} \psi \mathcal{R}(a+i b)^{m}(f) d m_{W}
$$

is stated and proved in Section 8 and Appendix B. Since the right-hand side of this lemma involves the supremum and Lipschitz norms of the argument $f$, we use mollification operators $\mathbb{M}_{\epsilon}$ like 9 in $\left[\mathrm{BL}\right.$ to replace the distribution $f \in \mathcal{B}$ by the function $\mathbb{M}_{\epsilon}(f)$. The operators $\mathbb{M}_{\epsilon}$ are introduced and studied in Section 7 . Finally, in Section 9, putting together Lemma 8.1 and Proposition 5.1, we first prove the spectral gap (claims (a) and (b) of Theorem 1.4) of the generator $X$ and then, applying recent work of Butterley [Bu], we easily obtain Theorem 1.2 (and thus Corollary 1.3 on exponential mixing) and claim (c) of Theorem 1.4.

Notation. We use $A=C^{ \pm 1} B$ for $A, B \in \mathbb{R}$ and $C \geq 1$ to mean $\frac{|B|}{C} \leq|A| \leq C|B|$. Let $0<\beta \leq 1$ be a real number and $f$ be a real or complex-valued function on a metric space $\left(W, d_{W}\right)$, then $\mathcal{C}^{\beta}(f)$ (or $\mathcal{C}_{W}^{\beta}(f)$ for emphasis) denotes the $\beta$-Hölder constant of $f$ and $|f|_{\infty}$ (or $|f|_{L^{\infty}(W)}$ for emphasis) denotes the supremum of $f$ on $W$.

\section{Definition OF the NORMS}

2.1. Stable and unstable cones for the flow. We recall the (well known) stable cones for the map $T$ and use them to define stable cones for the flow $\Phi_{t}$. We first need to introduce standard notation. In the coordinates $(x, y, \omega)$ and $(r, \varphi)$ from Subsection 1.1, the flow is given between collisions by

$$
\Phi_{t}\left(x_{0}, y_{0}, \omega_{0}\right)=\left(x_{t}, y_{t}, \omega_{t}\right)=\left(x_{0}+t \cos \omega_{0}, y_{0}+t \sin \omega_{0}, \omega_{0}\right)
$$

and at collisions by $\left(x^{+}, y^{+}, \omega^{+}\right)=\left(x^{-}, y^{-}, \omega^{-}+\pi-2 \varphi\right)$, where $(r, \varphi)$ is the collision point.

We will also work with the following coordinates in the tangent space. Setting $\hat{x}$ and $\hat{y}$ to be unit vectors in the $x$ and $y$ directions, respectively, we define

$$
\hat{\eta}=(\cos \omega) \hat{x}+(\sin \omega) \hat{y}, \quad \hat{\xi}=(-\sin \omega) \hat{x}+(\cos \omega) \hat{y} .
$$

The corresponding coordinates $(d \eta, d \xi, d \omega)$ in the tangent space are called the Jacobi coordinates for the flow. In these coordinates, the linearized flow is given between collisions by ([CM, (3.26)])

$$
D_{Z} \Phi_{t}(d \eta, d \xi, d \omega)=\left(d \eta_{t}, d \xi_{t}, d \omega_{t}\right)=(d \eta, d \xi+t d \omega, d \omega)
$$

\footnotetext{
${ }^{9}$ Note however that the Sobolev nature of the norms in BL made the corresponding estimates much easier than in the present work.
} 
and at collisions by (see [CM, (3.28)]) $\left(d \eta^{+}, d \xi^{+}, d \omega^{+}\right)=\left(d \eta^{-},-d \xi^{-},-d \omega^{-}-\frac{2 \mathcal{K}(r)}{\cos \varphi} d \xi^{-}\right)$, where the collision is at the point $(r, \varphi)$.

A crucial feature is that the $d \xi d \omega$-plane perpendicular to the flow direction is preserved under the flow. In addition, the flow preserves horizontal planes $(\omega=$ const.) between collisions. We will use these facts to introduce after Definition 2.7 the family $\mathcal{W}^{s}$ of stable curves with which our Banach norms will be defined.

For $Z \in Q \times \mathbb{S}^{1}$, let $P^{+}(Z)$ denote the point in $\mathcal{M}=\left(\cup_{i} \Gamma_{i}\right) \times \mathbb{S}^{1}$ that represents the first collision of $Z$ with the one of the scatterers under the flow. Similarly, denote by $P^{-}(Z) \in \mathcal{M}$ the point of first collision of $Z$ with one of the scatterers under the backwards flow. Since $P^{+}(Z)$ and $P^{-}(Z)$ lie in the phase space for the map $T$, we will sometimes refer to these points in the map coordinates $z=(r, \varphi)$. In these coordinates, we have the following standard choice of globally defined unstable and stable cones for the map. (See e.g. [CM, (4.13)].)

$$
\begin{aligned}
C_{z}^{u} & =\left\{(d r, d \varphi) \in \mathbb{R}^{2}: \mathcal{K}_{\min } \leq \frac{d \varphi}{d r} \leq \mathcal{K}_{\max }+\frac{1}{\tau_{\min }}\right\} \\
C_{z}^{s} & =\left\{(d r, d \varphi) \in \mathbb{R}^{2}:-\mathcal{K}_{\min } \geq \frac{d \varphi}{d r} \geq-\mathcal{K}_{\max }-\frac{1}{\tau_{\min }}\right\} .
\end{aligned}
$$

We have (see [CM, Exercise 4.19]) for any $z=(r, \varphi) \in \mathcal{M}$

$$
D_{z} T\left(C_{z}^{u}\right) \subset \operatorname{int} C_{T(z)}^{u} \cup\{0\} \quad D_{z} T^{-1}\left(C_{z}^{s}\right) \subset \operatorname{int} C_{T^{-1}(z)}^{s} \cup\{0\} .
$$

To translate these cones to the Jacobi coordinates for the flow, we use [CM, eq. (2.12)] (note that $\psi$ there denotes $\psi=\pi / 2-\varphi$, with $d \psi=-d \varphi$, see also [CM, eq. (3.19), p.51]), which yields

$$
d \omega=-\mathcal{K}(r) d r+d \varphi \quad d \xi=\cos \varphi d r-\tau(Z) d \omega
$$

for a tangent vector $(0, d \xi, d \omega)$, perpendicular to the flow at a point $Z=(x, y, \omega)$ that collides with the boundary at the point $(r, \varphi)$ at time $\tau(Z)$, i.e., $P^{+}(Z)=(r, \varphi)$. The slope of this vector in the $d \xi d \omega$-plane is thus

$$
\frac{d \omega}{d \xi}=\frac{1}{\frac{\cos \varphi}{-\mathcal{K}(r)+\frac{d \varphi}{d r}}-\tau(Z)}
$$

Flowing $C_{z}^{s}$ backward from the boundary yields the following family of stable cones for the flow

$$
C^{s}(Z)=\left\{(0, d \xi, d \omega):-\frac{1}{\frac{\cos \varphi\left(P^{+}(Z)\right)}{\mathcal{K}\left(P^{+}(Z)\right)+\mathcal{K}_{\min }}+\tau(Z)} \geq \frac{d \omega}{d \xi} \geq-\frac{1}{\frac{\cos \varphi\left(P^{+}(Z)\right)}{\mathcal{K}\left(P^{+}(Z)\right)+\mathcal{K}_{\max }+\frac{1}{\tau_{\min }}}+\tau(Z)}\right\}
$$

Thus the stable cones flow backwards only until the first collision $P^{-}(Z)$ and then are reset.

Remark 2.1. Equivalently, in terms of $1 /$ (wave front curvature), the cone $C^{s}(Z)=$

$$
\left\{(0, d \xi, d \omega):-\frac{\cos \varphi\left(P^{+}(Z)\right)}{\mathcal{K}\left(P^{+}(Z)\right)+\mathcal{K}_{\max }+\frac{1}{\tau_{\min }}}-\tau(Z) \geq \frac{d \xi}{d \omega} \geq-\frac{\cos \varphi\left(P^{+}(Z)\right)}{\mathcal{K}\left(P^{+}(Z)\right)+\mathcal{K}_{\min }}-\tau(Z)\right\} .
$$

Analogously, using [CM], eq. (2.11)] the unstable cones for the flow are defined by flowing $C_{z}^{u}$ forwards,

$$
C^{u}(Z)=\left\{(0, d \xi, d \omega): \frac{1}{\frac{\cos \varphi\left(P^{-}(Z)\right)}{\mathcal{K}\left(P^{-}(Z)\right)+\mathcal{K}_{\min }}+\tau^{-}(Z)} \leq \frac{d \omega}{d \xi} \leq \frac{1}{\frac{\cos \varphi\left(P^{-}(Z)\right)}{\mathcal{K}\left(P^{-}(Z)\right)+\mathcal{K}_{\max }+\frac{1}{\tau_{\min }}}+\tau^{-}(Z)}\right\}
$$

where $\tau^{-}(Z)$ denotes the time of first collision starting at $Z$ under the backwards flow. Just like $\tau$, the function $\tau^{-}$is (piecewise) $1 / 2$ Hölder continuous.

Note that both families of cones are bounded away from the horizontal in the $\xi \omega$-plane due to the finite horizon condition. Near tangential collisions $(\tau=\cos \varphi=0)$, both families of cones 
squeeze to a line (approaching the vertical direction), but never at the same point: $C^{u}(Z)$ is arbitrarily close to vertical just after nearly tangential collisions, while $C^{s}(Z)$ is bounded away from the vertical at these points due to the fact that $\tau_{\min }>0$. The roles of $C^{s}(Z)$ and $C^{u}(Z)$ are reversed just before nearly tangential collisions. Thus $C^{s}(Z)$ and $C^{u}(Z)$ are uniformly transverse in the phase space for the flow.

Our cones are planar and therefore trivially have empty interior in $\mathbb{R}^{3}$, contrary to the flow cones used in $\mathrm{BL}$. See also Remark 2.3 . Like in $[\mathrm{BL}]$ (see also $[\mathrm{BuL}]$ ), we get strict contraction only for large enough times:

Lemma 2.2. For any $Z \in \Omega_{0}$, and any $t \geq 0$, we have

$$
D_{Z} \Phi_{t}\left(C^{u}(Z)\right) \subseteq C^{u}\left(\Phi_{t}(Z)\right), \quad D_{Z} \Phi_{-t}\left(C^{s}(Z)\right) \subseteq C^{s}\left(\Phi_{-t}(Z)\right) .
$$

In addition, if $t>\tau_{\max }$ then (slightly abusing notation, "int" refers to the interior of the cone in the plane $d \eta=0$ ) for any $Z \in \Omega_{0}$

$$
D_{Z} \Phi_{t}\left(C^{u}(Z)\right) \subset\{0\} \cup \operatorname{int} C^{u}\left(\Phi_{t}(Z)\right), \quad D_{Z} \Phi_{-t}\left(C^{s}(Z)\right) \subset\{0\} \cup \operatorname{int} C^{s}\left(\Phi_{-t}(Z)\right) .
$$

Proof. First note that the planes $\mathcal{T}_{Z}^{\perp} \Omega_{0} \subset \mathcal{T}_{Z} \Omega_{0}$ defined by $d \eta=0$ are perpendicular to the flow and preserved by the flow, see [CM, Cor. 3.12, (3.14)]. So we may restrict $D_{Z} \Phi_{t}$ to these planes.

We consider the statements for unstable cones; the others are similar.

If $0<t<\tau(Z)$, then the claim immediately follows from the definition of the cones since $D_{Z} \Phi_{t}\left(C^{u}(Z)\right)=C^{u}\left(\Phi_{t}(Z)\right)$. This applies to any factor $\left[\begin{array}{ll}1 & t \\ 0 & 1\end{array}\right]$ between collisions in the decomposition [CM, (3.29)] for $D_{Z} \Phi_{t}$ restricted to $\mathcal{T}_{Z}^{\perp} \Omega_{0}$.

For the action at a collision, using [CM, (3.29)] again, (2.2) and the definition of the unstable cone give (with $Z^{-}$and $Z^{+}$representing the moments just before and just after collision, respectively)

$$
-\left[\begin{array}{cc}
1 & 0 \\
2 \mathcal{K} / \cos \varphi & 1
\end{array}\right]\left(C^{u}\left(Z^{-}\right)\right) \subset\{0\} \cup \operatorname{int}\left(C^{u}\left(Z^{+}\right)\right) .
$$

where we view the cones (and take interior) as subsets of $\mathcal{T}_{Z^{ \pm}}^{\perp} \Omega_{0}$. There is at least one collision factor in the decomposition if $t>\tau_{\max }$, ending the proof.

2.2. Admissible stable and unstable curves for the flow. Following [DZ1, a $\mathcal{C}^{1}$ curve $V$ in $\mathcal{M}$ is called a stable curve for the map $T$ if the tangent vector at each point $z$ in $V$ lies in $C_{z}^{s}$. We call a $\mathcal{C}^{1}$ curve $W \subset \Omega_{0}$ a stable curve for the flow if at every point $Z \in W$, the tangent vector $\mathcal{T}_{Z} W$ to $W$ lies in $C^{s}(Z)$. An essential property of stable curves $W$ is

$$
\#\left(\bar{W} \cap \partial \Omega_{0}\right) \leq 2
$$

(where $\bar{W}$ denotes the closure of $W$ ) since either both the scatterer and the wavefront corresponding to a stable curve are convex in opposition, or the curvature of a stable curve is bounded away (for this claim, see e.g., the paragraph containing (8.6) ) from the curvature of the scatterer. Our definition also implies that a stable curve $W$ is perpendicular to the flow, which is essential in the following remark:

Remark 2.3 (Checking that stable curves lie in the kernel of the contact form). In the Dolgopyat estimate we will use that stable curves $W$ belong to the kernel of the invariant contact form $\boldsymbol{\alpha}$.

\footnotetext{
10 Our curves $W$ are diffeomorphic to a bounded open interval, in particular relatively open at endpoints. However, we require the $\mathcal{C}^{r}(r=\alpha, \beta, 1,2)$ norms of functions supported on $W$ to be bounded in the sense of Whitney on the closed interval.
} 
In 11 the present billiard case, $\boldsymbol{\alpha}=p \mathbf{d} q$ (see e.g. [Ar. App. 4]), and since the velocity $p=v=\left(v_{1}, v_{2}\right)=(\cos \omega, \sin \omega)$, we have

$$
\boldsymbol{\alpha}=\cos \omega \mathbf{d} x+\sin \omega \mathbf{d} y,
$$

so that $\mathbf{d} \boldsymbol{\alpha}=-\sin \omega \mathbf{d} \omega \wedge \mathbf{d} x+\cos \omega \mathbf{d} \omega \wedge \mathbf{d} y$ and

$$
\boldsymbol{\alpha} \wedge \mathbf{d} \boldsymbol{\alpha}=\mathbf{d} x \wedge \mathbf{d} \omega \wedge \mathbf{d} y .
$$

In particular, $\boldsymbol{\alpha}$ coincides with the Jacobi coordinate $(0,0, d \eta)$ corresponding to the velocity (i.e., flow) direction (referring - again - to [CM. Cor. 3.12, (3.14)]), and the stable and unstable cones lie in the kernel of the contact form $\boldsymbol{\alpha}$, as desired. Note that by definition of contact, there is no surface tangent to the kernel of $\boldsymbol{\alpha}$ on an open set (in fact, the maximal dimension of a manifold everywhere tangent to this kernel is one).

Remark 2.4 (Putting the contact form in standard form). If we replace the coordinates $(x, y, \omega) \in$ $Q \times \mathbb{S}^{1}$ by $(\omega, \tilde{\xi}, \tilde{\eta}) \in \mathbb{S}^{1} \times \widetilde{Q}$ where

$$
\omega=\omega, \quad \tilde{\xi}=-x \sin \omega+y \cos \omega, \quad \tilde{\eta}=x \cos \omega+y \sin \omega,
$$

(defining $\widetilde{Q}$ by the above), then inverting gives $\omega=\omega, x=\tilde{\eta} \cos \omega-\tilde{\xi} \sin \omega, y=\tilde{\eta} \sin \omega+\tilde{\xi} \cos \omega$, so that

$$
\begin{aligned}
\boldsymbol{\alpha}= & \cos \omega \mathbf{d} x+\sin \omega \mathbf{d} y \\
= & \cos \omega[\cos \omega \mathbf{d} \tilde{\eta}-\tilde{\eta} \sin \omega \mathbf{d} \omega-\sin \omega \mathbf{d} \tilde{\xi}-\tilde{\xi} \cos \omega \mathbf{d} \omega] \\
& \quad+\sin \omega[\sin \omega \mathbf{d} \tilde{\eta}+\tilde{\eta} \cos \omega \mathbf{d} \omega+\cos \omega \mathbf{d} \tilde{\xi}-\tilde{\xi} \sin \omega \mathbf{d} \omega]=\mathbf{d} \tilde{\eta}-\tilde{\xi} \mathbf{d} \omega .
\end{aligned}
$$

Therefore, the contact form $\boldsymbol{\alpha}$ takes the standard form $\boldsymbol{\alpha}_{\mathbf{0}}=\mathbf{d} \tilde{\eta}-\tilde{\xi} \mathbf{d} \omega$ in the coordinates $(\omega, \tilde{\xi}, \tilde{\eta})$. Note that the change of coordinates (2.6) in the phase space of the flow is the same as the one used to construct the Jacobi coordinates $(d \eta, d \xi, d \omega)$, except that (2.1) corresponded to changing variables in the tangent space. So there is no contradiction between the fact that $\boldsymbol{\alpha}=$ $\mathbf{d} \tilde{\eta}-\tilde{\xi} \mathbf{d} \omega$ and the fact that $\boldsymbol{\alpha}$ vanishes on those vectors so that $d \eta=0$. (Indeed, vectors which are perpendicular to $(1,0,0)$ in $(d \eta, d \xi, d \omega)$ coordinates are perpendicular to $(\cos \omega, \sin \omega, 0)$ in $(d x, d y, d \omega)$ coordinates by definition of $d \eta$. Now given the definition of $\boldsymbol{\alpha}$, we have (in $(d x, d y, d \omega)$ coordinates), $\boldsymbol{\alpha}(v)=\cos \omega \mathbf{d} x(v)+\sin \omega \mathbf{d} y(v)=(\cos \omega, \sin \omega, 0) \cdot v$, so that $\boldsymbol{\alpha}(v)=0$ if and only if $v$ is perpendicular to $(d \eta, 0,0)$.)

In Sections 6 and 8 (analogously to what was done in $[\mathrm{BL}]$ ), we will need coordinate charts and local coordinates $\left(x^{u}, x^{s}, x^{0}\right)$ such that at the origin of such charts, $(1,0,0)$ lies in the unstable cone, $(0,1,0)$ lies in the stable cone, and $(0,0,1)$ is the flow direction, while

$$
\boldsymbol{\alpha}=\mathbf{d} x^{0}-x^{s} \mathbf{d} x^{u} .
$$

(We call such charts cone-compatible Darboux charts.) The above does not seem to hold for the coordinates $(\omega, \tilde{\xi}, \tilde{\eta})$ (recall the conditions on the slope $d \omega / d \xi$ defining $C^{u}$ and $C^{s}$ ), but it can be achieved by applying a "symplectic" change of coordinates, as in [BL, Lemma A.4],

$$
\left.\omega=A x^{s}+B x^{u}, \quad \tilde{\xi}=C x^{s}+D x^{u}, \quad \tilde{\eta}=x^{0}+\frac{A C}{2}\left(x^{s}\right)^{2}+A D x^{s} x^{u}+\frac{B D}{2}\left(x^{d}\right)^{2}\right),
$$

with $A D-B C=1$, for $A, B, C, D$ real-valued functions. This is possible, since inverting the above gives

$$
\left.x^{s}=D \omega-B \tilde{\xi}, \quad x^{u}=-C \omega+A \tilde{\xi}, \quad x^{0}=\tilde{\eta}-\left[\frac{A C}{2}\left(x^{s}\right)^{2}+A D x^{s} x^{u}+\frac{B D}{2}\left(x^{u}\right)^{2}\right)\right],
$$

\footnotetext{
${ }^{11}$ We use a bold upright symbol $\mathbf{d}$ to denote the exterior derivative. This should not be confused with the $d$ used for coordinates in a vector $(d r, d \varphi),(d \eta, d \xi, d \omega)$, adopted from [CM].
} 
and we may find $A>0, B>0, C<0, D>0$, with $A D-B C=1$ so that $(D,-B)$ lies in the stable cone while $(-C, A)$ lies in the unstable cone. In order to be able to choose the functions $A, B, C$, and $D$ (and thus the charts) in a $\mathcal{C}^{2}$ way, we shall discard small neighborhoods where the cone directions do not behave in a nice way. This will be performed in Remarks 6.1 and 8.3.

The homogeneity layer decay rate $k^{-\chi}$ with $\chi=2$ in Definition 2.5 below is standard in the literature. Letting $\chi>1$ tend to 1 gives better estimates, as we explain in Remark 5.3 .

Definition 2.5 (Homogeneity strips). To control distortion, we define the usual homogeneity strips for the map by

$$
\mathbb{H}_{k}=\left\{(r, \varphi) \in \mathcal{M}: \pi / 2-1 / k^{2} \leq \varphi \leq \pi / 2-1 /(k+1)^{2}\right\}
$$

for $k \geq k_{0}$, and similarly for $\mathbb{H}_{-k}$ near $\varphi=-\pi / 2$. We also put

$$
\mathbb{H}_{0}=\left\{(r, \varphi) \in \mathcal{M}:-\pi / 2+1 / k_{0}^{2} \leq \varphi \leq \pi / 2-1 / k_{0}^{2}\right\} .
$$

Finally, we set for $|k| \geq k_{0}$

$$
\mathbb{S}_{k}=\left\{(r, \varphi):|\varphi|=\pi / 2-k^{-2}\right\}, \text { and } \mathbb{S}=\cup_{|k| \geq k_{0}} \mathbb{S}_{k} .
$$

$A$ subset $Z$ of $\mathcal{M}$ is called homogeneous if $Z \subset \mathbb{H}_{k_{1}}$ for some $k_{1} \in\{0\} \cup\left\{k \geq k_{0}\right\}$.

Definition 2.6 (Singularity sets). We define $\mathcal{S}_{0}=\partial \mathcal{M}=\{|\varphi|=\pi / 2\}$, and, inductively, for $n \geq 1$, the iterated singularity sets

$$
\mathcal{S}_{n}=\mathcal{S}_{n-1} \cup T^{-1}\left(\mathcal{S}_{n-1}\right), \quad \mathcal{S}_{-n}=\mathcal{S}_{-(n-1)} \cup T\left(\mathcal{S}_{-(n-1)}\right) .
$$

The extended singularity sets are defined for $n \geq 0$ by

$$
\mathcal{S}_{n}^{\mathbb{H}}=\mathcal{S}_{n} \cup\left(\cup_{m=0}^{n} T^{-m} \mathbb{S}\right), \quad \mathcal{S}_{-n}^{\mathbb{H}}=\mathcal{S}_{-n} \cup\left(\cup_{m=0}^{n} T^{m} \mathbb{S}\right) .
$$

Recall that $\mathcal{S}_{-n} \backslash \mathcal{S}_{0}$ consists of finitely many smooth unstable curves while $\mathcal{S}_{n} \backslash \mathcal{S}_{0}$ consists of finitely many smooth stable curves, see [CM, (2.19), (2.27), Proposition 4.45, Exercise 4.46,(5.14), (5.15) §5.4].

Definition 2.7. An admissible stable curve for $L>0$ and $B>0$ is a subset $W$ of $\Omega_{0}$, satisfying the following conditions:

(W1) $W$ is a stable curve for the flow. (This implies that $P^{+}(W)$ is a stable curve for the map, by definition of the stable cones. If $P^{-}(W)$ intersects $n \geq 1$ scatterers, then $P^{-}(W)$ is a union of $n$ stable curves for the map, using $P^{-}(W)=T^{-1}\left(P^{+}(W)\right)$.)

(W2) $W$ has length at most $L$ and is $\mathcal{C}^{2}$, with curvature bounded by $B>0$. for the map $T$ (in particular, $\cos \varphi \neq 0$ for any $\left.(r, \varphi) \in P^{+}(W)\right)$.

If in addition $P^{+}(W)$ is homogeneous, then $W$ is called homogeneous.

We denote by $\mathcal{W}^{s}$ the set of all admissible stable curves for fixed $L=L_{0}$ and $B=B_{0}$, where $L_{0}$ is chosen in Lemma 3.8 below (determined in particular by the complexity condition for the map, see also Definition 3.1) and $B_{0}$ is chosen after Lemma 3.2. The set $\mathcal{W}^{s}$ is not empty. In fact by simply flowing a homogeneous stable curve for the map backwards under the flow, we may construct homogeneous flow stable curves $W$. Unstable curves and (homogeneous) admissible unstable curves are defined similarly.

Definition 2.8 (Distances $d_{\mathcal{W}^{s}}\left(W_{1}, W_{2}\right)$ between stable curves). Let $W_{1}, W_{2} \in \mathcal{W}^{s}$ be two stable curves. If there does not exist any unstable curve $W_{1,2}^{u}$ (for the flow) so that $W_{1,2}^{u} \cap W_{1}$ and $W_{1,2}^{u} \cap W_{2}$ are both nonempty, we set $d_{\mathcal{W}^{s}}\left(W_{1}, W_{2}\right)=\infty$. If there exists 12 such a curve $W_{1,2}^{u}$, writing the projections $P^{+}\left(W_{i}\right)$ as graphs $P^{+}\left(W_{i}\right)=\left\{\left(r, \varphi_{i}(r)\right): r \in I_{i}\right\}$ over some interval $I_{i}$,

12 This holds for example if $W_{1} \cap W_{2} \neq \emptyset$. It does not hold if $W_{2}=\Phi_{t}\left(W_{1}\right)$. 
we define $d_{\mathcal{W}^{s}}\left(W_{1}, W_{2}\right)=\left|I_{1} \triangle I_{2}\right|+\left|\varphi_{1}-\varphi_{2}\right|_{\mathcal{C}^{1}\left(I_{1} \cap I_{2}\right)}$, where $\triangle$ denotes the symmetric difference of two intervals, and $|\varphi|_{\mathcal{C}^{\ell(I)}}=\sum_{k=0}^{\ell} \sup _{I}\left|D^{k} \varphi\right|$.

Note that this distance stated in terms of the projected curves is the distance used in [DZ1] between stable curves for the map (with the small difference that the curves there were assumed to be homogeneous). For future reference, we denote this distance between projected curves by $\tilde{d}_{\mathcal{W}^{s}}\left(P^{+}\left(W_{1}\right), P^{+}\left(W_{2}\right)\right)$.

Remark 2.9. Our distance between $W_{1}$ and $W_{2}$ is based on $P^{+}\left(W_{1}\right)$ and $P^{+}\left(W_{2}\right)$, so if $W_{1}$ and $W_{2}$ are two halves of the same stable curve split at a single point making a collision, then the distance between them is infinite in our norms since $P^{+}\left(W_{1}\right)$ and $P^{+}\left(W_{2}\right)$ lie on different scatterers (or the opposite side of the same scatterer, but then the distance is still of order one).

Definition 2.10 (Distance $d\left(\psi_{1}, \psi_{2}\right)$ between test functions). Let $\psi_{1}$ and $\psi_{2}$ be supported on stable curves $W_{1}$ and $W_{2}$, respectively. Since $P^{+}\left(W_{i}\right)$ is represented by the graph of $\varphi_{i}$ over $I_{i}$, let $G_{W_{i}}(r)=\left(r, \varphi_{i}(r)\right)$ denote this graph and $S_{W_{i}}(r)=\Phi_{-t(r)} \circ G_{W_{i}}(r)$ denote the natural map from $I_{i}$ to $W_{i}$ defined by the flow. We use this map to define the distance between test functions by $d\left(\psi_{1}, \psi_{2}\right)=\left|\psi_{1} \circ S_{W_{1}}-\psi_{2} \circ S_{W_{2}}\right|_{\mathcal{C}^{0}\left(I_{1} \cap I_{2}\right)}$ when $I_{1} \cap I_{2} \neq \emptyset$. If $I_{1} \cap I_{2}=\emptyset$, we set $d\left(\psi_{1}, \psi_{2}\right)=\infty$, i.e., we simply do not compare these functions.

(The notation for $S_{W_{i}}$ and in particular the map $\Phi_{-t(r)}$ used in the definition above hides the change of coordinates from $(r, \varphi)$ to $(x, y, \omega)$. The corresponding Jacobian is a product of the Jacobian of the flow between collisions and that of the change of variables. See Sections 3 and 4.)

2.3. The norms $|\cdot|_{w},\|\cdot\|_{s},\|\cdot\|_{u},\|\cdot\|_{0}$, and the spaces $\mathcal{B}$ and $\mathcal{B}_{w}$. Given $W \in \mathcal{W}^{s}$ and $0<\beta<1$, we define the Hölder norm of a test function $\psi$ by $|\psi|_{\mathcal{C}^{\beta}(W)}=|\psi|_{\mathcal{C}^{0}(W)}+\mathcal{C}_{W}^{\beta}(\psi)$. Letting $\mathcal{C}^{1}(W)$ denote the set of continuously differentiable functions on $W$, we define the spaces $\mathcal{C}^{\beta}(W)$ to be the closure of $\mathcal{C}^{1}(W)$ in the $|\cdot|_{\mathcal{C}^{\beta}(W)}$ norm 13 .

Now ${ }^{14}$ fix $0<\alpha \leq 1 / 3$. For $f \in \mathcal{C}^{1}\left(\Omega_{0}\right)$, define the weak norm of $f$ by

$$
|f|_{w}=\sup _{W \in \mathcal{W}^{s}} \sup _{\substack{\psi \in \mathcal{C}^{\alpha}(W) \\|\psi|_{\mathcal{C}^{\alpha}(W)} \leq 1}} \int_{W} f \psi d m_{W}
$$

where $d m_{W}$ denotes arclength along $W$.

Now choose $1<q<\infty$ and $0<\beta<\min \{\alpha, 1 / q\}$. For $f \in \mathcal{C}^{1}\left(\Omega_{0}\right)$, define the strong stable norm of $f$ by

$$
\|f\|_{s}=\sup _{W \in \mathcal{W}^{s}} \sup _{\substack{\psi \in \mathcal{C}^{\beta}(W) \\|W|^{1 / q}|\psi|_{\mathcal{C}^{\beta}(W)} \leq 1}} \int_{W} f \psi d m_{W}
$$

13 This space $\mathcal{C}^{\beta}(W)$ coincides with the "little Hölder space" noted $b_{\infty, \infty}^{\beta}$ in [RS, Prop. 2.1.2, Def. 2.1.3.1] obtained by taking the closure of $\mathcal{C}^{\infty}$ in the $|\cdot|_{\mathcal{C}^{\beta}(W)}$ norm, it is strictly smaller than the set of all Hölder continuous functions with exponent $\beta$, but contains all Hölder continuous functions with exponent $\beta^{\prime}>\beta$.

${ }^{14}$ The value $1 / 3$ is determined by the choice (2.7) of homogeneity layers, see Lemma 3.5. Replacing $k^{2}$ by $k^{\chi}$ for $\chi>1$ replaces the bound $\alpha \leq 1 / 3$ by $\alpha \leq 1 /(\chi+1)$.

15 In view of (4.5), it will be natural to further restrict to those $\beta$ which satisfy in addition $\beta \leq 1-1 / q$. 
Choose $0<\gamma \leq \min \{1 / q, \alpha-\beta\}<1 / 3$. Define the unstable norm of $f$ by 16

$$
\|f\|_{u}=\sup _{\varepsilon>0} \sup _{\substack{W_{1}, W_{2} \in \mathcal{W}^{s} \\ d_{\mathcal{W}^{s}}\left(W_{1}, W_{2}\right) \leq \varepsilon}} \sup _{\substack{\psi_{i} \in \mathcal{C}^{\alpha}\left(W_{i}\right) \\ \psi_{i} \mid \mathcal{C}^{\alpha}\left(W_{i}\right) \leq 1 \\ d\left(\psi_{1}, \psi_{2}\right)=0}} \varepsilon^{-\gamma}\left|\int_{W_{1}} f \psi_{1} d m_{W_{1}}-\int_{W_{2}} f \psi_{2} d m_{W_{2}}\right| .
$$

We define the neutral norm of $f$ by

$$
\|f\|_{0}=\left.\sup _{W \in \mathcal{W}^{s}} \sup _{\substack{\psi \in \mathcal{C}^{\alpha}(W) \\|\psi|_{\mathcal{C}^{\alpha}(W)} \leq 1}} \int_{W} \partial_{t}\left(f \circ \Phi_{t}\right)\right|_{t=0} \psi d m_{W} .
$$

Finally, define the strong norm of $f$ by

$$
\|f\|_{\mathcal{B}}=\|f\|_{s}+c_{u}\|f\|_{u}+\|f\|_{0},
$$

where $c_{u}>0$ is a constant to be chosen in Section 5

We will prove in Lemma 3.9 that there exists a constant $C$ so that for any $f \in \mathcal{C}^{1}\left(\Omega_{0}\right)$

$$
\|f\|_{\mathcal{B}} \leq C|f|_{\mathcal{C}^{1}\left(\Omega_{0}\right)} .
$$

In the other direction, we relate our norms to the dual of Hölder spaces. For $0<r<1$ and any $f \in L^{\infty}\left(\Omega_{0}\right)$, set

$$
|f|_{\left(\mathcal{C}^{r}\right)^{*}}=\sup \left\{\int f \psi d m: \psi \in \mathcal{C}^{r}\left(\Omega_{0}\right),|\psi|_{\mathcal{C}^{r}\left(\Omega_{0}\right)} \leq 1\right\}<\infty .
$$

where $\mathcal{C}^{r}\left(\Omega_{0}\right)$ is the closure of $\mathcal{C}^{1}$ for the $\mathcal{C}^{r}$ norm (little Hölder space). The following estimate follows from the role of $0<\beta<\alpha$ in our definition (the proof is given in Section 7).

Lemma 2.11 (Relation with the dual of $\mathcal{C}^{r}\left(\Omega_{0}\right)$ ). There exists $C \geq 1$ so that

$$
|f|_{\left(\mathcal{C}^{\alpha}\left(\Omega_{0}\right)\right)^{*}} \leq C|f|_{w}, \quad|f|_{\left(\mathcal{C}^{\beta}\left(\Omega_{0}\right)\right)^{*}} \leq C\|f\|_{s} \quad \forall f \in \mathcal{C}^{0}\left(\Omega_{0}\right) .
$$

We introduce the subspace $\mathcal{C}_{\sim}^{0}$ of those continuous functions on $\Omega_{0}$ which can be viewed as continuous functions on the quotient space $\Omega=\Omega_{0} / \sim$ :

$$
\mathcal{C}_{\sim}^{0}=\mathcal{C}^{0}(\Omega)=\left\{f \in \mathcal{C}^{0}\left(\Omega_{0}\right) \mid f(Z)=f(Y) \text { if } Z \sim Y\right\}
$$

It is well known that $\Phi_{t}$ is continuous on the topological metric quotient 17 space $\Omega$ see e.g. $\mathrm{CM}$, Exercise 2.27]. This implies that if $f \in \mathcal{C}_{\sim}^{0}$ then $f \circ \Phi_{-t} \in \mathcal{C}_{\sim}^{0}$ for all $t \in \mathbb{R}$. We can now introduce our main Banach spaces:

Definition 2.12 (Banach spaces $\left.\mathcal{B}_{w}, \mathcal{B}\right)$. Let $\mathcal{B}_{w}$ be the completion with respect to $|\cdot|{ }_{w}$ of the set $\left\{\left.f \in \mathcal{C}_{\sim}^{0}|| f\right|_{w}<\infty\right\}$. Let $\mathcal{B} \subset \mathcal{B}_{w}$ be the completion for the norm $\|\cdot\|_{\mathcal{B}}$ of

$$
\left\{\mathcal{L}_{t} f \mid t \geq 0, \quad f \in \mathcal{C}_{\sim}^{0} \cap \mathcal{C}^{2}\left(\Omega_{0}\right)\right\} .
$$

The apparently contrived definition of $\mathcal{B}$ will ensure joint continuity of $(t, f) \mapsto \mathcal{L}_{t}(f)$ in Lemma 4.6, adapting [BL, (3.9)-(3.12)].

Lemma 2.11 implies that $\mathcal{B} \subset\left(\mathcal{C}^{\beta}\left(\Omega_{0}\right)\right)^{*}$ and $\mathcal{B}_{w} \subset\left(\mathcal{C}^{\alpha}\left(\Omega_{0}\right)\right)^{*}\left(\right.$ defining $\left(\mathcal{C}^{r}\right)^{*}$ to be the closure of $\mathcal{C}^{1}$ functions $f$ for the $\left(\mathcal{C}^{r}\right)^{*}$ norm), while Lemma 7.5 implies that $\mathcal{C}^{1}\left(\Omega_{0}\right) \subset \mathcal{B}$. Clearly, $\mathcal{B} \subset \mathcal{B}_{w}$ (we shall see in Lemma 3.10 that the embedding is compact). The injectivity of the various embeddings is summarized in Lemma 3.9 .

\footnotetext{
16 In previous works, $d\left(\psi_{1}, \psi_{2}\right)=0$ was replaced by $d_{\beta}\left(\psi_{1}, \psi_{2}\right) \leq \varepsilon$ where $\beta>0$ and the distance used the $\mathcal{C}^{\beta}$ instead of the $\mathcal{C}^{0}$ norm. The two formulations are equivalent by the triangle inequality, using the strong stable norm, and since $d\left(\psi_{1}, \psi_{2}\right)=0$ implies $d_{\beta}\left(\psi_{1}, \psi_{2}\right)=0$.

17 The requirement $\|f\|_{0}<\infty$ essentially implies that $f$ is in some kind of Sobolev space with positive exponent in the flow direction, thus continuous almost everywhere on the quotient $\Omega$. We shall neither prove nor use this.
} 
Note for further use that, by the group property of the flow, $f \circ \Phi_{r+s+\delta}-f \circ \Phi_{r+s}=\left(f \circ \Phi_{s+\delta}-\right.$ $\left.f \circ \Phi_{s}\right) \circ \Phi_{r}$ for any $f \in \mathcal{C}_{\sim}^{0}$, so that, whenever both sides of the identity below are well-defined,

$$
\left.\partial_{t}\left(f \circ \Phi_{t}\right)\right|_{t=r+s}(Z)=\left.\left(\partial_{t} f \circ \Phi_{t}\right)\right|_{t=s} \circ \Phi_{r}(Z) .
$$

\section{Preliminary Lemmas}

In this section, we establish some basic facts about expansion and control of complexity and prove several key properties of our Banach spaces.

3.1. Growth and distortion. When we flow a stable curve $W$ backwards under the flow, $\Phi_{-t}(W)$ may be cut by singularity curves or undergo large expansion at collisions. For fixed $t>0$ and $Z$, the $t$-trace of $Z$ (on the collision space $\mathcal{M}$, up to time $t$ ) is the set $\left\{\Phi_{s}(Z) \cap \mathcal{M}, s \in(0, t]\right\}$. (Note that $\Phi_{-t}(W)$ has a cusp at $Z$ if the $t$-trace of $Z$ contains a tangential collision.) Recall [CM, p.76] that the trace of $E \subset \Omega_{0}$ is simply $P^{+}(E)$.

Definition 3.1 (The partition $\mathcal{G}_{t}(W)$ ). For $t \geq 0$ and $W \in \mathcal{W}^{s}$, we partition $\Phi_{-t}(W)$ as follows 18 First, we partition $\Phi_{-t}(W)$ at any points whose $t$-trace intersects either the boundary of one of the homogeneity strips $\mathbb{H}_{k}$ or a tangential collision point $\{\varphi= \pm \pi / 2\}$. Second, if any component defined thus far was subdivided at a previous time $s$, for $0<s<t$ due to growing to length greater than $L_{0}$, we continue to consider $\Phi_{-t}(W)$ to be subdivided at the image of this point under $\Phi_{s-t}$. Finally, if a component reaches length $=L_{0}$, we subdivide it into two curves of length $L_{0} / 2$. The countable collection of components of $\Phi_{-t}(W)$ defined in this way is denoted by $\widehat{\mathcal{G}}_{t}(W)$. If one of the elements of $\widehat{\mathcal{G}}_{t}(W)$ is in the midst of a collision at time $t$, i.e., if this component intersects the boundary of one of the scatterers (such an intersection can contain at most two points, since both the scatterer and the stable curve (a diverging wavefront under the backwards flow) are convex in opposition, recall (2.5)), then we split this component into two or three pieces temporarily: one or two curves consisting of points that have just completed a collision and one curve consisting of points that are about to make a collision. This refined set of components is denoted by $\mathcal{G}_{t}(W)$.

Note that for $s>0$, if $W_{i} \in \widehat{\mathcal{G}}_{t}(W)$, then $\Phi_{-s}\left(W_{i}\right)$ is a union of elements in $\widehat{\mathcal{G}}_{t+s}(W)$. However, if $W_{i} \in \mathcal{G}_{t}(W)$, then $\Phi_{-s}\left(W_{i}\right)$ may not be a union of elements of $\mathcal{G}_{t+s}(W)$ if $W_{i}$ was part of an curve undergoing a collision at time $t$ which has since completed its collision without crossing any boundaries of homogeneity strips. In this case, $\Phi_{-s}\left(W_{i}\right)$ is contained in an element of $\widehat{\mathcal{G}}_{t+s}(W)$.

The following lemma proves the invariance of the family $\mathcal{W}^{s}$ under $\Phi_{-t}, t>0$. While the ideas in the proof are by now well-known, we include the lemma since we are not aware of a published proof that includes the family of flow-stable curves $\mathcal{W}^{s}$ we have introduced here. Our proof follows the approach of [CM, § 4.6], which contains many of the ideas we will need.

Lemma 3.2 (Invariance of stable curves). For $W \in \mathcal{W}^{s}$ and $t \geq 0$, let $\mathcal{G}_{t}(W)=\left\{W_{i}\right\}_{i}$. Then $W_{i} \in \mathcal{W}^{s}$ for each $i$.

Proof. Due to the inductive definition of $\mathcal{G}_{t}(W)$ and the invariance (Lemma 2.2) of the stable cones, each element $W_{i}$ satisfies condition (W1) required for $\mathcal{W}^{s}$. The one point to prove is (W2): The curvature of such curves remains uniformly bounded for all times. At collisions,

$$
\frac{d \omega^{+}}{d \xi^{+}}=\frac{d \omega^{-}}{d \xi^{-}}+\frac{2 \mathcal{K}(r)}{\cos \varphi}
$$

(cf. [CM, eq. (3.33)]), and this quantity represents the curvature of the wave front (a stable curve $W$ projected onto the $x y$-plane). Yet the expansion in the $\omega$ direction is of the same order, so as

\footnotetext{
18 Whenever we partition a curve into finitely or countably many subcurves, we drop the (at most countably many) division points.
} 
we will show below, the curvature of $W$ in $\mathbb{R}^{3}$ remains uniformly bounded, even near tangential collisions.

We begin by parametrizing a stable curve $W$ approaching a collision (under the backwards flow) by points $Z_{s}=\left(x_{s}, y_{s}, \omega_{s}\right)$ and define $Z_{s t}=\Phi_{-t}\left(Z_{s}\right)$. The points $Z_{s t}$ fill a $2 D$ surface in $\Omega_{0}$ and we choose the interval of $t$ to be large enough that we follow $W$ through precisely one collision. We assume that $\Phi_{-t}(W)$ is still smooth after completing the collision.

We will denote derivatives with respect to $t$ by dots and those with respect to $s$ by primes. Define $u=-x^{\prime} \sin \omega+y^{\prime} \cos \omega$. Then since $\left(x^{\prime}, y^{\prime}, \omega^{\prime}\right)$ remains perpendicular to the velocity vector, $(\cos \omega, \sin \omega, 0)$, we have

$$
u^{2}=\left(x^{\prime}\right)^{2}+\left(y^{\prime}\right)^{2} \text { and } u^{\prime}=-x^{\prime \prime} \sin \omega+y^{\prime \prime} \cos \omega .
$$

We will need the following relations, which follow from [CM, eqs. (4.25),(4,26)],

$$
\omega^{\prime}=u B \quad \text { and } \quad \omega^{\prime \prime}=u^{\prime} B+u^{2} \frac{d B}{d \xi},
$$

where $B=d \omega / d \xi$ represents the curvature of $W$ projected onto the $x y$-plane.

We will denote the curvature of $W$ in $\mathbb{R}^{3}$ by $\kappa$. By definition,

$$
\kappa^{2}=\frac{\left(\omega^{\prime} y^{\prime \prime}-y^{\prime} \omega^{\prime \prime}\right)^{2}+\left(\omega^{\prime} x^{\prime \prime}-x^{\prime} \omega^{\prime \prime}\right)^{2}+\left(x^{\prime} y^{\prime \prime}-y^{\prime} x^{\prime \prime}\right)^{2}}{\left(\left(x^{\prime}\right)^{2}+\left(y^{\prime}\right)^{2}+\left(\omega^{\prime}\right)^{2}\right)^{3}} .
$$

Notice that the arclength factor satisfies $\left(x^{\prime}\right)^{2}+\left(y^{\prime}\right)^{2}+\left(\omega^{\prime}\right)^{2}=u^{2}\left(1+B^{2}\right)$, by (3.1) and (3.2). We consider each term in the numerator separately. For the first term, using (3.1) and again (3.2), we let $B_{\xi}$ denote $d B / d \xi$ and write

$$
\begin{aligned}
\omega^{\prime} y^{\prime \prime}-y^{\prime} \omega^{\prime \prime} & =B\left(-x^{\prime} \sin \omega+y^{\prime} \cos \omega\right) y^{\prime \prime}-y^{\prime}\left(u^{\prime} B+u^{2} B_{\xi}\right) \\
& =B\left(-x^{\prime} y^{\prime \prime} \sin \omega+y^{\prime} y^{\prime \prime} \cos \omega\right)+\left(y^{\prime} x^{\prime \prime} \sin \omega-y^{\prime} y^{\prime \prime} \cos \omega\right) B-y^{\prime} u^{2} B_{\xi} \\
& =B \sin \omega\left(y^{\prime} x^{\prime \prime}-x^{\prime} y^{\prime \prime}\right)-y^{\prime} u^{2} B_{\xi} .
\end{aligned}
$$

A similar calculation for the second term yields,

$$
\omega^{\prime} x^{\prime \prime}-x^{\prime} \omega^{\prime \prime}=B \cos \omega\left(y^{\prime} x^{\prime \prime}-x^{\prime} y^{\prime \prime}\right)-x^{\prime} u^{2} B_{\xi} .
$$

Substituting these relations into the expression for $\kappa^{2}$ and using again (3.1) and that $x^{\prime} \cos \omega+$ $y^{\prime} \sin \omega=0$, we obtain $\kappa^{2}=$

$$
\begin{aligned}
& \frac{B^{2}\left(\sin ^{2} \omega\left(y^{\prime} x^{\prime \prime}-x^{\prime} y^{\prime \prime}\right)^{2}+\left(y^{\prime}\right)^{2} u^{4} B_{\xi}^{2}+\cos ^{2} \omega\left(y^{\prime} x^{\prime \prime}-x^{\prime} y^{\prime \prime}\right)^{2}\right)+\left(x^{\prime}\right)^{2} u^{4} B_{\xi}^{2}+\left(x^{\prime} y^{\prime \prime}-y^{\prime} x^{\prime \prime}\right)^{2}}{\left(\left(x^{\prime}\right)^{2}+\left(y^{\prime}\right)^{2}+\left(\omega^{\prime}\right)^{2}\right)^{3}} \\
& =\frac{\left(1+B^{2}\right)\left(x^{\prime} y^{\prime \prime}-y^{\prime} x^{\prime \prime}\right)^{2}+u^{6} B_{\xi}^{2}}{u^{6}\left(1+B^{2}\right)^{3}}=\frac{B^{2}}{\left(1+B^{2}\right)^{2}}+\frac{B_{\xi}^{2}}{\left(1+B^{2}\right)^{3}},
\end{aligned}
$$

where in the last equality, we have used the fact that the curvature of the planar wavefront satisfies $B^{2}=\left(x^{\prime} y^{\prime \prime}-y^{\prime} x^{\prime \prime}\right)^{2} / u^{6}$ by definition. The first term in the expression for $\kappa^{2}$ is bounded by $1 / 4$ for all $B \in \mathbb{R}$. The second term is bounded above by $B_{\xi}^{2} / B^{6}$ and we use [CM, eqs. (4.36), (4.38)] to conclude that $B_{\xi} / B^{3}$ remains uniformly bounded for all time, even after undergoing collisions arbitrarily close to tangential.

A corollary of the above proof (in particular [CM, eq. (4.38)]) is that there exists a constant $B_{0}>0$ such that if $W$ is a stable curve with curvature less than $B_{0}$, then each smooth component of $\Phi_{-t}(W)$ also has curvature less than $B_{0}$. We fix this choice of $B_{0}$ in the definition of $\mathcal{W}^{s}$.

If $W$ is a flow-stable curve, $Z \in W$, and $t \in \mathbb{R}$, we denote by $J_{W} \Phi_{t}(Z)$ the Jacobian of $\Phi_{t}$ from $W$ to $\Phi_{t}(W)$, at $Z$, with respect to the arclength measure on $W$. Similarly, if $n$ is an integer 
then $J_{U} T^{n}(z)$ denotes the Jacobian of $T^{n}$ from the map-stable curve $U$ to $T^{n}(U)$, at $z \in U$, with respect to the arclength measure on $U$.

Lemma 3.3. There exists $C_{0}>0$, such that for all $W \in \mathcal{W}^{s}$, all $t>0$, all $W_{i} \in \mathcal{G}_{t}(W)$, and all $Z \in W_{i}$; the Jacobian $J_{W_{i}} \Phi_{t}(Z)=C_{0}^{ \pm 1} J_{P^{+}\left(W_{i}\right)} T^{n}\left(P^{+}(Z)\right)$, where $n$ is the number of collisions of $P^{+}(Z)$ before time $t$.

Proof. Set $V_{i}=\Phi_{t} W_{i}$. Since $J_{V_{i}} \Phi_{-t}\left(\Phi_{t} Z\right)=\left(J_{W_{i}} \Phi_{t}(Z)\right)^{-1}$, it is equivalent to estimate $J_{V_{i}} \Phi_{-t}$.

First we show that for $V \in \mathcal{W}^{s}$, the expansion from $P^{+}(V)$ to $V$ given by the inverse of the flow from $Z \in V$ to $P^{+}(V)$ is of order 1 even though $P^{+}(V)$ may not be homogeneous. (The proof of this is a refinement of [CM, Exercise 3.15], taking advantage of the fact that we only consider stable curves.)

For $Z \in V$, setting $P^{+}(Z)=z=(r, \varphi)$, we let $J_{P^{+}(V)} \Phi_{-\tau(Z)}$ denote the Jacobian of the map from $P^{+}(V)$ (in $(r, \varphi)$ coordinates) to $V$ (in $(x, y, \omega)$ coordinates) under the backwards flow. Let $d z=(d r, d \varphi)$ denote the image of a vector $d Z=(0, d \xi, d \omega) \in C^{s}(Z)$. Recalling (2.3), and since $\|d Z\|^{2}=(d \xi)^{2}+(d \omega)^{2}$, we obtain

$$
\|d Z\|^{2}=(d r)^{2}\left[\left(\cos \varphi+\tau(Z) \mathcal{K}(r)-\tau(Z) \frac{d \varphi}{d r}\right)^{2}+\left(\mathcal{K}(r)-\frac{d \varphi}{d r}\right)^{2}\right] .
$$

On the one hand, (3.3) implies $\|d Z\|^{2} \leq C\|d z\|^{2}$, where $C$ is a constant depending on $\mathcal{K}_{\max }, \tau_{\max }$ and $1 / \tau_{\min }$, since $(d r, d \varphi) \in C_{z}^{s}$. On the other hand, since $\frac{d \varphi}{d r}<0$, we get that $\|d Z\|^{2} \geq$

$$
(d r)^{2}\left(\mathcal{K}(r)-\frac{d \varphi}{d r}\right)^{2} \geq(d r)^{2}\left[\mathcal{K}(r)^{2}+\left(\frac{d \varphi}{d r}\right)^{2}\right]=\mathcal{K}(r)^{2}(d r)^{2}+(d \varphi)^{2} \geq \min \left\{\mathcal{K}_{\text {min }}^{2}, 1\right\}\|d z\|^{2}
$$

proving the claim.

Next, we fix $Z \in V_{i}$ as above and decompose its past orbit as follows, let $z_{0}=P^{+}(Z)$ and $z_{-i}=T^{-1} z_{-i+1}$ for $i=1, \ldots n$, where $n$ is the number of collisions between $Z$ and $\Phi_{-t}(Z)$. Then, letting $d Z_{0}$ denote a vector tangent to $V$ at $Z$ perpendicular to the flow and $d Z_{-t}$ denote its image under $D \Phi_{-t}$, we have

$$
J_{V_{i}} \Phi_{-t}(Z)=\frac{\left\|d Z_{-t}\right\|}{\left\|d Z_{0}\right\|}=\frac{\left\|d Z_{-t}\right\|}{\left\|d z_{-n}\right\|} \frac{\left\|d z_{-n}\right\|}{\left\|d z_{0}\right\|} \frac{\left\|d z_{0}\right\|}{\left\|d Z_{0}\right\|},
$$

where $d z_{i}$ represents the image of $d Z_{0}$ at $z_{i}$. The first and third factors above are of order 1 by the previous claim. The middle factor is precisely $J_{P^{+}\left(V_{i}\right)} T^{-n}\left(P^{+}(Z)\right)$. Now since $J_{V_{i}} \Phi_{-t}\left(\Phi_{t} Z\right)=$ $\left(J_{W_{i}} \Phi_{t}(Z)\right)^{-1}$ and $J_{P^{+}\left(V_{i}\right)} T^{-n}\left(P^{+}\left(\Phi_{t} Z\right)\right)=\left(J_{P^{+}\left(W_{i}\right)} T^{n}\left(P^{+}(Z)\right)\right)^{-1}$, the lemma is proved.

The following lemma will be the key to the bounded distortion Lemma 3.5. (Note that the exponent $1 / 2$ is intrinsic to the billiard and cannot be improved.)

Lemma 3.4. For any $W \in \mathcal{W}^{s}$, let $S_{W}(r)=\Phi_{-t(r)} \circ G_{W}(r)$ denote the map from $I_{W}$ to $W$ defined at the end of Subsection 2.2. There exists $C>0$, depending only on the table and choice of $L_{0}$ and $B_{0}$, such that $\left|\ln J S_{W}\right|_{\mathcal{C}^{1 / 2}\left(I_{W}\right)} \leq C$ and $\left|\ln J S_{W}^{-1}\right|_{\mathcal{C}^{1 / 2}(W)} \leq C$, where $J S_{W}^{ \pm 1}$ denotes the Jacobian of $S_{W}^{ \pm 1}$.

Proof. As in the proof of Lemma 3.3, for $Z \in W$ and $z=P^{+}(Z)$, let $J_{P^{+}(W)} \Phi_{-\tau(Z)}(z)$ denote the Jacobian of the map from $P^{+}(W)$ to $W$ under the backwards flow. Then for $r \in I_{W}$ with $z=G_{W}(r)$, we have $J S_{W}(r)=J_{P^{+}(W)} \Phi_{-\tau(Z)}(z) J G_{W}(r)$.

First note that (as in [DZ1, eq. (3.18)]),

$$
\left|J G_{W}\right|=\sqrt{1+\left(\frac{d \varphi_{W}}{d r}\right)^{2}} \leq C_{g}:=\sqrt{1+\left(\mathcal{K}_{\max }+\tau_{\min }^{-1}\right)^{2}} .
$$


Also $J G_{W} \geq 1$ and since by (3.3) and the estimates following, $J_{P^{+}(W)} \Phi_{-\tau(Z)}(z)$ is uniformly bounded above and below away from zero, the $\mathcal{C}^{0}$ bound is proved. We proceed to estimate the Hölder constant. Since $J G_{W}$ is $\mathcal{C}^{1}$ using (3.5) and the fact that $W$ has bounded curvature, it remains to estimate $\mathcal{C}^{1 / 2}\left(\ln J_{P^{+}(W)} \Phi_{-\tau}\right)$.

Let $Z_{1}, Z_{2} \in W$ and set $z_{j}=\left(r_{j}, \varphi_{j}\right)=P^{+}\left(Z_{j}\right), j=1,2$. Using again (3.3), we have

$$
\begin{aligned}
& \ln \frac{J_{P^{+}(W)}\left(\Phi_{-\tau\left(Z_{1}\right)}\left(z_{1}\right)\right)}{J_{P^{+}(W)}\left(\Phi_{-\tau\left(Z_{2}\right)}\left(z_{2}\right)\right)}=\ln \frac{\left|d z_{2}\right|}{\left|d z_{1}\right|} \\
& \quad+\frac{1}{2} \ln \frac{\left(d r_{1}\right)^{2}\left[\left(\cos \varphi_{1}+\tau\left(Z_{1}\right) \mathcal{K}\left(r_{1}\right)-\tau\left(Z_{1}\right) \frac{d \varphi_{1}}{d r_{1}}\right)^{2}+\left(\mathcal{K}\left(r_{1}\right)-\frac{d \varphi_{1}}{d r_{1}}\right)^{2}\right]}{\left(d r_{2}\right)^{2}\left[\left(\cos \varphi_{2}+\tau\left(Z_{2}\right) \mathcal{K}\left(r_{2}\right)-\tau\left(Z_{2}\right) \frac{d \varphi_{2}}{d r_{2}}\right)^{2}+\left(\mathcal{K}\left(r_{2}\right)-\frac{d \varphi_{2}}{d r_{2}}\right)^{2}\right]},
\end{aligned}
$$

where $d z_{j}=\left(d r_{j}, d \varphi_{j}\right)$ denotes the tangent vector to $P^{+}(W)$ at $z_{j}$. Without loss of generality, we may set $d r_{1}=d r_{2}=1$. For the first term on the right-hand side, we have

$$
\ln \frac{\left|d z_{2}\right|}{\left|d z_{1}\right|}=\frac{1}{2} \ln \left(\frac{1+\left(d \varphi_{2}\right)^{2}}{1+\left(d \varphi_{1}\right)^{2}}\right) \leq \frac{1}{2}\left(d \varphi_{2}-d \varphi_{1}\right)\left(d \varphi_{2}+d \varphi_{1}\right) \leq C d\left(z_{1}, z_{2}\right),
$$

where in the last step, we used that $d \varphi_{1}, d \varphi_{2}$ are uniformly bounded by definition of $C_{z}^{s}$ as well as the fact that $\left|\frac{d \varphi_{1}}{d r_{1}}-\frac{d \varphi_{2}}{d r_{2}}\right| \leq C d\left(z_{1}, z_{2}\right)$ since $P^{+}(W)$ has uniformly bounded curvature. Using again (3.3) and the fact that $W$ has uniformly bounded curvature according to (W2), we get $C d_{W}\left(Z_{1}, Z_{2}\right) \leq d\left(z_{1}, z_{2}\right) \leq C^{-1} d_{W}\left(Z_{1}, Z_{2}\right)$, for some $C>0$.

Now we turn to the second term on the right-hand side of $(3.6)$. Since $J_{P^{+}(W)} \Phi_{-\tau(Z)}(z)$ is bounded away from 0 and all the functions appearing in the numerator and denominator of the right-hand side are uniformly bounded, it suffices to consider the differences in each function of the right-hand side separately.

It is easy to verify by direct inspection (using that our billiard has finite horizon, while $P^{+}\left(Z_{1}\right)$ and $P^{+}\left(Z_{2}\right)$ lie in a connected component of the complement of the singularity set for $T$ in $\mathcal{M}$, and the curvatures of the scatterers are bounded above) that 19

$$
\left|\tau\left(Z_{1}\right)-\tau\left(Z_{2}\right)\right| \leq C d_{W}\left(Z_{1}, Z_{2}\right)^{1 / 2}
$$

Since $\mathcal{K}$ and $\cos \varphi$ are smooth functions of $r$ and $\varphi$, the differences in $\mathcal{K}$ and $\cos \varphi$ are Lipschitz in $d_{W}\left(Z_{1}, Z_{2}\right)$ as well. Putting these estimates together and using again that $d_{W}\left(Z_{1}, Z_{2}\right) \leq$ $C^{-1} d\left(z_{1}, z_{2}\right)$, we have that the Hölder constant $\mathcal{C}^{1 / 2}\left(\ln J_{P^{+}(W)} \Phi_{-\tau}\right) \leq C$ for some $C>0$, independent of $W$.

The estimate for the inverse follows similarly, using the fact that $J S_{W}^{-1}=\left(J S_{W}\right)^{-1} \circ S_{W}^{-1}$ and exploiting $d\left(z_{1}, z_{2}\right) \leq C^{-1} d_{W}\left(Z_{1}, Z_{2}\right)$.

In the following distortion bound, the exponent $1 / 3$ is a consequence of our choice of decay $1 / k^{2}$ for the homogeneity layers.

Lemma 3.5 (Bounded distortion). There exists $C_{d}>0$, such that for all $W \in \mathcal{W}^{s}, t \geq 0$, and $Z_{1}, Z_{2} \in W_{i} \in \mathcal{G}_{t}(W)$,

$$
\left|\frac{J_{W_{i}} \Phi_{t}\left(Z_{1}\right)}{J_{W_{i}} \Phi_{t}\left(Z_{2}\right)}-1\right| \leq C_{d} d_{W_{i}}\left(Z_{1}, Z_{2}\right)^{1 / 3} .
$$

Proof. Fixing $W \in \mathcal{W}^{s}$ and $t>0$, if $\Phi_{-s}(W), 0 \leq s \leq t$, has undergone no collisions then the required bound holds trivially due to the linearity of the flow between collisions. On the

19 The factor $1 / 2$ here is intrinsic and not related to the quadratic decay choice in (2.7). 
other hand, if $\Phi_{-t}(W)$ has undergone a collision, then $W_{i}$ is a homogeneous stable curve by construction of $\mathcal{G}_{t}(W)$.

Let $n$ denote the number of collisions from $W_{i}$ to $\Phi_{t}\left(W_{i}\right)$. We will use (3.4) in order to leverage the bounded distortion enjoyed by the collision map $T$. We begin with the first factor, which gives the Jacobian of the map from $P^{+}\left(W_{i}\right)$ to $W_{i}$ and is $J_{P^{+}\left(W_{i}\right)} \Phi_{-\tau(Z)}(z)$ in the notation of the proof of Lemma 3.4. This is log-Hölder with exponent $1 / 2$ by that lemma.

Comparable estimates hold for the last factor in (3.4), which represents the Jacobian of the map from $P^{+}\left(\Phi_{t}\left(W_{i}\right)\right)$ to $\Phi_{t}\left(W_{i}\right)$. Finally, since $P^{+}\left(\Phi_{t}\left(W_{i}\right)\right)=T^{n}\left(P^{+}\left(W_{i}\right)\right)$, the middle factor in (3.4) enjoys bounded distortion along stable curves with the Hölder exponent of $1 / 3$ [CM], Lemma 5.27]. Notice that for the stable Jacobian of the map to enjoy bounded distortion at the last iterate (from $T^{n-1}\left(P^{+}\left(W_{i}\right)\right)$ to $T^{n}\left(P^{+}\left(W_{i}\right)\right)$ ), it is essential that $T^{n-1}\left(P^{+}\left(W_{i}\right)\right.$ ) be a homogeneous stable curve, but not that $T^{n}\left(P^{+}\left(W_{i}\right)\right) \subset P^{+}(W)$ be homogeneous. This is because $J_{T^{n-1}\left(P^{+}\left(W_{i}\right)\right)} T\left(z_{1}\right)$ is proportional to $\cos \varphi\left(z_{1}\right)$, not $\cos \varphi\left(T\left(z_{1}\right)\right)$. Combining the estimates for these three factors completes the proof of the lemma.

Recall the hyperbolicity constant $\Lambda$ for the flow from (1.2). The following elementary lemma is one of the keys to exponential mixing:

Lemma 3.6 (Exponential decay of stable-Hölder constants under the flow). For any $0 \leq \kappa \leq 1$ there exists $C_{1}>0$ so that for any $W$, for each $\psi \in \mathcal{C}^{\kappa}(W)$, all $t>0$, and all $W_{i} \in \mathcal{G}_{t}(W)$,

$$
\mathcal{C}_{W_{i}}^{\kappa}\left(\psi \circ \Phi_{t}\right) \leq C_{1} \Lambda^{-t \kappa} \mathcal{C}_{W}^{\kappa}(\psi)
$$

Proof. This will be a consequence of Lemma 3.3, since for $Z_{1}, Z_{2} \in W_{i}$,

$$
\frac{\left|\psi \circ \Phi_{t}\left(Z_{1}\right)-\psi \circ \Phi_{t}\left(Z_{2}\right)\right|}{d_{W_{i}}\left(Z_{1}, Z_{2}\right)^{\kappa}}=\frac{\left|\psi \circ \Phi_{t}\left(Z_{1}\right)-\psi \circ \Phi_{t}\left(Z_{2}\right)\right|}{d_{W}\left(\Phi_{t}\left(Z_{1}\right), \Phi_{t}\left(Z_{2}\right)\right)^{\kappa}} \frac{d_{W}\left(\Phi_{t}\left(Z_{1}\right), \Phi_{t}\left(Z_{2}\right)\right)^{\kappa}}{d_{W_{i}}\left(Z_{1}, Z_{2}\right)^{\kappa}} \leq \mathcal{C}_{W}^{\kappa}(\psi) C \Lambda_{0}^{-n \kappa}
$$

where $n$ represents the number of collisions undergone by $W_{i}$ by time $t$ and we have used that $\Lambda_{0}$ is the minimum expansion factor for the map [CM, (4.19)]. The lemma follows since $\left\lfloor\frac{t}{\tau_{\max }}\right\rfloor-1 \leq$ $n \leq\left\lfloor\frac{t}{\tau_{\min }}\right\rfloor+1$.

We next present a growth lemma20 adapted from [DZ1, §3.2] which is a direct consequence of the one-step expansion [CM, Lemma 5.56]. Recall $L_{0}$ from the definition of $\mathcal{W}^{s}$.

Definition $3.7\left(\mathcal{I}_{t}(W)\right)$. For $W \in \mathcal{W}^{s}$ and $t \geq 0$, let $\mathcal{I}_{t}(W)$ be those elements $W_{i} \in \mathcal{G}_{t}(W)$ such that $\Phi_{s}\left(W_{i}\right)$ is contained in an element $V_{i}^{s} \in \widehat{\mathcal{G}}_{t-s}(W)$ with $\left|V_{i}^{s}\right|<L_{0} / 3$ for all $0 \leq s \leq t$. (The curves $\Phi_{t}\left(W_{i}\right)$ corresponding to $\mathcal{I}_{t}(W)$ are repeatedly cut by the singularities of $\Phi_{-t}$ without ever growing to size $L_{0} / 3$ before time $t$.)

Lemma 3.8 (Modified growth lemma). For any $\lambda \in\left(\Lambda_{0}^{-1 / \tau_{\max }}, 1\right)$, if $L_{0}$ is small enough, then there exists $C_{2} \geq 1$ such that for all $1 \leq q_{0} \leq \infty, W \in \mathcal{W}^{s}$, and $t \geq 0$,
(a) $\sum_{W_{i} \in \mathcal{I}_{t}(W)} \frac{\left|W_{i}\right|^{1 / q_{0}}}{|W|^{1 / q_{0}}}\left|J_{W_{i}} \Phi_{t}\right|_{\mathcal{C}^{0}\left(W_{i}\right)} \leq C_{2} \lambda^{t\left(1-1 / q_{0}\right)} ;$
(b) $\sum_{W_{i} \in \mathcal{G}_{t}(W)} \frac{\left|W_{i}\right|^{1 / q_{0}}}{|W|^{1 / q_{0}}}\left|J_{W_{i}} \Phi_{t}\right|_{\mathcal{C}^{0}\left(W_{i}\right)} \leq C_{2}$.

Proof. By Lemma 3.4, there exists $C_{3} \geq 1$ such that $\left|W_{i}\right|=C_{3}^{ \pm 1}\left|P^{+}\left(W_{i}\right)\right|$. We next invoke the one-step expansion for the map from [CM, Lemma 5.56]. Using [CM], (3.31), (5.39), (5.36)],

${ }^{20}$ In Step 1 of Section 6] we shall apply a growth lemma [CM, Theorem 5.52] directly. 
(since $\mathcal{B}_{1}^{-}=1 /\left(\tau_{0}+1 / \mathcal{B}_{0}^{+}\right)$, we have $\mathcal{B}_{1}^{-} \tau_{\min } \leq 1$ ), we set $\tilde{L}_{0}=C_{3}^{-1} L_{0}$ and for $\lambda_{1} \in\left(\Lambda_{0}^{-1}, 1\right)$, choose $L_{0} \leq \tau_{\min } / 4$ sufficiently small that the one-step expansion for the map satisfies

$$
\sup _{|V| \leq \tilde{L}_{0}} \sum_{i} \frac{\left|T V_{i}\right|_{*}}{\left|V_{i}\right|_{*}} \leq \lambda_{1},
$$

where $V$ is a (not necessarily homogeneous) map stable curve, $V_{i}$ are the homogeneous components of $T^{-1} V$, and $|\cdot|_{*}$ denotes length in the adapted metric defined in [CM, Section 5.10]: For a tangent vector $(d r, d \varphi)$, define $\|(d r, d \varphi)\|_{*}=\frac{\mathcal{K}+\left|\frac{d \varphi}{d r}\right|}{\sqrt{1+\left(\frac{d \varphi}{d r}\right)^{2}}}\|(d r, d \varphi)\|$, where $\mathcal{K}$ denotes the curvature at the given point.

Fix $n$ and consider curves $W_{i} \in \mathcal{G}_{t}(W)$ undergoing $n$ collisions between $\Phi_{t}\left(W_{i}\right)$ and $W$. Then $P^{+}\left(W_{i}\right)$ is contained in an element of $\widetilde{\mathcal{G}}_{n}\left(P^{+}(W)\right)$, where $\widetilde{\mathcal{G}}_{n}\left(P^{+}(W)\right)$ are the connected homogeneous components of $T^{-n}\left(P^{+}(W)\right)$, defined inductively as in [DZ1, Sect 3.2]. If $W_{i}$ does not have an endpoint on a scatterer (i.e., is not in the midst of a collision), then the correspondence is one to one. If $W_{i}$ is part of a longer element making a collision, then there are at most two such $W_{i}$ for each element of $\widetilde{\mathcal{G}}_{n}\left(P^{+}(W)\right)$, due to the fact that at most two points of a diverging wave front can be in contact with a scatterer at one time (this divides the curve into at most three pieces, but note that in this case, two of the three will have made $n$ collisions, while one of the three will have made $n+1$ collisions, and so will be grouped in the $(n+1)$ th generation, $\widetilde{\mathcal{G}}_{n+1}\left(P^{+}(W)\right)$.

Since the expansion rates for the flow are comparable to those for the map by Lemma 3.3. and we have just seen that there are at most two components of $\mathcal{G}_{t}(W)$ for each component of the corresponding partition $\widetilde{\mathcal{G}}_{n}\left(P^{+}(W)\right)$ into homogeneous components of $P^{+}(W)$ for the map, we will use the analogous estimates for the map from [DZ1] to prove the growth lemma. The added complication here is that the components of $\mathcal{G}_{t}(W)$ may have undergone different numbers of collisions and so they belong to an assortment of generations for the discrete time map.

Fix $W \in \mathcal{W}^{s}$, and $W_{i} \in \mathcal{G}_{t}(W)$ for $t>0$. Let $V=P^{+}(W)$ and $V_{i}=P^{+}\left(W_{i}\right)$. For (a), by [DZ1, (3.2), (3.3), Lemma 3.1] (choosing $\delta_{1}=\tilde{L}_{0} / 3$ in the notation used there), there exists $C>0$ such that for any $n \in \mathbb{N}$,

$$
\sum_{V_{i} \in \widetilde{\mathcal{I}}_{n}(V)}\left|J_{V_{i}} T^{n}\right|_{\mathcal{C}^{0}\left(V_{i}\right)} \leq C \lambda_{1}^{n}
$$

where $\widetilde{\mathcal{G}}_{k}(V)$ are the smooth components of $T^{-k}(V)$ defined in the beginning of the proof and $\widetilde{\mathcal{I}}_{n}(V)$ denotes the set of smooth components $V_{i}$ of $T^{-n}(V)$ such that $T^{j}\left(V_{i}\right)$ never belongs to a curve in $\widetilde{\mathcal{G}}_{n-j}(V)$ that has length greater than $\tilde{L}_{0} / 3$ for $0 \leq j \leq n$. Since the number of collisions undergone by each $W_{i}$ before time $t$ may vary, we denote by $\mathcal{I}_{t, n}(W)$ those components of $\mathcal{I}_{t}(W)$ which experience $n$ collisions by time $t$. Note that $\left\lfloor\frac{t}{\tau_{\max }}\right\rfloor-1 \leq n \leq\left\lfloor\frac{t}{\tau_{\min }}\right\rfloor+1$. Thus by (3.9) and Lemma 3.3 .

$$
\begin{aligned}
\sum_{W_{i} \in \mathcal{I}_{t}(W)}\left|J_{W_{i}} \Phi_{t}\right|_{\mathcal{C}^{0}\left(W_{i}\right)} & =\sum_{n=\left\lfloor\frac{t}{\tau_{\max }}\right\rfloor-1}^{\left\lfloor\frac{t}{\tau_{\min }}\right\rfloor+1} \sum_{W_{i} \in \mathcal{I}_{t, n}(W)}\left|J_{W_{i}} \Phi_{t}\right|_{\mathcal{C}^{0}\left(W_{i}\right)} \\
& \leq 2 C_{0} \sum_{n=\left\lfloor\frac{t}{\tau_{\min }}\right\rfloor+1} C \lambda_{1}^{n} \leq C^{\prime} \lambda_{1}^{t / \tau_{\max }}
\end{aligned}
$$


proving part (a) of the lemma for $1 / q_{0}=0$ with $\lambda=\lambda_{1}^{1 / \tau_{\max }}$. Part (a) of the lemma for $1 / q_{0} \in(0,1]$ follows by an application of the Hölder inequality, as in [DZ1, Lemma 3.3]:

$$
\begin{aligned}
\sum_{W_{i} \in \mathcal{I}_{t}(W)} \frac{\left|W_{i}\right|^{\frac{1}{q_{0}}}}{|W|^{\frac{1}{q_{0}}}}\left|J_{W_{i}} \Phi_{t}\right|_{\mathcal{C}^{0}\left(W_{i}\right)} & \leq\left(\sum_{W_{i} \in c I_{t}(W)} \frac{\left|W_{i}\right|}{|W|}\left|J_{W_{i}} \Phi_{t}\right|_{\mathcal{C}^{0}\left(W_{i}\right)}\right)^{\frac{1}{q_{0}}}\left(\sum_{W_{i} \in \mathcal{I}_{t}(W)}\left|J_{W_{i}} \Phi_{t}\right|_{\mathcal{C}^{0}\left(W_{i}\right)}\right)^{1-\frac{1}{q_{0}}} \\
& \leq\left(1+C_{d}\right)^{1 / q_{0}}\left(C^{\prime} \lambda_{1}^{t / \tau_{\max }}\right)^{1-1 / q_{0}}
\end{aligned}
$$

where we have used that $\left|J_{W_{i}} \Phi_{t}\right|_{\mathcal{C}^{0}\left(W_{i}\right)} \leq\left(1+C_{d}\right) \frac{\left|\Phi_{t}\left(W_{i}\right)\right|}{\left|W_{i}\right|}$ by Lemma 3.5, and $\sum_{i} \frac{\left|\Phi_{t}\left(W_{i}\right)\right|}{|W|} \leq 1$.

To prove part (b), we shall adapt the argument of [DZ1, Lemma 3.2]. First, we subdivide the time interval $[0, t]$ into times $t_{k}=t-k \tau_{\min }, k=0, \ldots,\left\lfloor t / \tau_{\min }\right\rfloor$. Using these subintervals, we group the components $W_{i} \in \mathcal{G}_{t}(W)$ into "most recent long ancestors," defining sets $L_{k}(W, t)$, $k=0, \ldots,\left\lfloor t / \tau_{\text {min }}\right\rfloor$, and grouping in $A_{t}(U)$ those $W_{i} \in \mathcal{G}_{t}(W)$ so that $U \in L_{k}(W, t)$, as follows. For each $W_{i}^{t} \in \mathcal{G}_{t}(W)$ and each $k$, we have $\Phi_{k \tau_{\min }}\left(W_{i}^{t}\right) \subset W_{j}^{t_{k}}$ for some $W_{j}^{t_{k}} \in \widehat{\mathcal{G}}_{t_{k}}(W)$. If $W_{j}^{t_{k}}$ is undergoing a collision, we can adjust $t_{k}$ (for that $W_{j}^{t_{k}}$ only) by adding a small time $\left|\tilde{\delta}\left(k, W_{i}^{t}\right)\right|<L_{0}$ so that $W_{j}^{t_{k}+\tilde{\delta}} \in \mathcal{G}_{t_{k}+\tilde{\delta}}(W)$ is an admissible stable curve. Note that even with this small correction, the times $t_{k}$ are still at least $\tau_{\min } / 2$ apart since $L_{0}$ has been chosen $<\tau_{\min } / 4$. We say that such a curve $W_{j}^{t_{k}}$ is the most recent long ancestor of $W_{i}^{t}$ if $\left|P^{+}\left(W_{j}^{t_{k}}\right)\right| \geq L_{0} / 3$ and $k \geq 0$ is the least such $k$ with this property for $W_{i}^{t}$. In this case, we put $i \in A_{t}\left(W_{j}^{t_{k}}\right)$ and $j \in L_{k}(W, t)$. If no such $k$ exists, then by definition, $W_{i}^{t} \in \mathcal{I}_{t}(W)$ (that we denote by $i \in \mathcal{I}_{t}(W)$ for simplicity). Using this grouping, we estimate,

$$
\sum_{W_{i}^{t} \in \mathcal{G}_{t}(W)}\left|J_{W_{i}^{t}} \Phi_{t}\right|_{\mathcal{C}^{0}\left(W_{i}^{t}\right)}=\sum_{k=0}^{\left\lfloor t / \tau_{\min }\right\rfloor} \sum_{j \in L_{k}(W, t)} \sum_{i \in A_{t}\left(W_{j}^{t}\right)}\left|J_{W_{i}^{t}} \Phi_{t}\right|_{\mathcal{C}^{0}\left(W_{i}^{t}\right)}+\sum_{i \in \mathcal{I}_{t}(W)}\left|J_{W_{i}^{t}} \Phi_{t}\right|_{\mathcal{C}^{0}\left(W_{i}^{t}\right)} .
$$

The sum over $\mathcal{I}_{t}(W)$ is exponentially small in $t$ by (a), so we focus on the sum over $k$. For each $W_{j}^{t_{k}}$, we have $\left|J_{W_{i}^{t}} \Phi_{t}\right|_{\mathcal{C}^{0}\left(W_{i}^{t}\right)} \leq\left|J_{W_{i}^{t}} \Phi_{k \tau_{\min }}\right|_{\mathcal{C}^{0}\left(W_{i}^{t}\right)}\left|J_{W_{j}^{t}} \Phi_{t_{k}}\right|_{\mathcal{C}^{0}\left(W_{j}^{t_{k}}\right)}$. Thus, using part (a) of the lemma from time $t_{k}$ to time $t$,

$$
\begin{aligned}
\sum_{i \in A_{t}\left(W_{j}^{t} k\right)}\left|J_{W_{i}^{t}} \Phi_{t}\right|_{\mathcal{C}^{0}\left(W_{i}^{t}\right)} & \leq\left|J_{W_{j}^{t} k} \Phi_{t_{k}}\right|_{\mathcal{C}^{0}\left(W_{j}^{t}\right)} \sum_{i \in A_{t}\left(W_{j}^{t}\right)}\left|J_{W_{i}^{t}} \Phi_{k \tau_{\min }}\right|_{\mathcal{C}^{0}\left(W_{i}^{t}\right)} \\
& \leq\left|J_{W_{j}^{t} k} \Phi_{t_{k}}\right|_{\mathcal{C}^{0}\left(W_{j}^{t}\right)} C \lambda^{k \tau_{\min }}
\end{aligned}
$$

Using this estimate, plus the fact that $\left|J_{W_{j}^{t_{k}}} \Phi_{t_{k}}\right|_{\mathcal{C}^{0}\left(W_{j}^{t_{k}}\right)} \leq C_{d} \frac{\left|\Phi_{t_{k}}\left(W_{j}^{t_{k}}\right)\right|}{\left|W_{j}^{t_{k}}\right|} \leq C L_{0}^{-1}\left|\Phi_{t_{k}}\left(W_{j}^{t_{k}}\right)\right|$ by bounded distortion (Lemma 3.5), we have

$$
\sum_{W_{i}^{t} \in \mathcal{G}_{t}(W)}\left|J_{W_{i}^{t}} \Phi_{t}\right|_{\mathcal{C}^{0}\left(W_{i}^{t}\right)} \leq \sum_{k=0}^{\left\lfloor t / \tau_{\min }\right\rfloor} \sum_{j \in L_{k}(W, t)} C L_{0}^{-1}\left|\Phi_{t_{k}}\left(W_{j}^{t_{k}}\right)\right| \lambda^{k \tau_{\min }}+C \lambda^{t}
$$

For each $k$, the sum over $j$ is at most $|W|$ since $\cup_{j} \Phi_{t_{k}}\left(W_{j}^{t_{k}}\right)$ is a disjoint union, and the sum over $k$ is uniformly bounded in $t$, proving part (b) for $1 / q_{0}=0$. Part (b) for $1 / q_{0}>0$ now follows from a Hölder inequality as in part (a).

${ }^{21}$ We write $t_{k}$ for $t_{k}+\tilde{\delta}\left(k, W_{i}^{t}\right)$, for simplicity. 
3.2. Embeddings of smooth functions in $\mathcal{B}$. Compactness of $\mathcal{B}$ in $\mathcal{B}_{w}$. Recalling $\mathcal{C}_{\sim}^{0}$ from (2.9), it is convenient to introduce two auxiliary Banach spaces:

The space $\mathcal{B}^{0}$ is the completion with respect to $\|\cdot\|_{\mathcal{B}}$ of

$$
\left\{f \in \mathcal{C}^{0}\left(\Omega_{0}\right) \mid\|f\|_{\mathcal{B}}<\infty\right\} .
$$

The space $\mathcal{B}_{\sim}^{0}$ is the completion with respect to $\|\cdot\|_{\mathcal{B}}$ of

$$
\left\{f \in \mathcal{C}_{\sim}^{0} \mid\|f\|_{\mathcal{B}}<\infty\right\} .
$$

These definitions ensure that $\mathcal{B} \subset \mathcal{B}_{\sim}^{0}$ and $\mathcal{C}_{\sim}^{0} \cap \mathcal{C}^{1} \subset \mathcal{B}_{\sim}^{0}$. Also, $\mathcal{B}_{\sim}^{0} \cap \mathcal{C}_{\sim}^{0}$ is dense in $\mathcal{B}_{\sim}^{0}$ and similarly for $\mathcal{B}_{w}$. This will allow us to define transfer operators $\mathcal{L}_{t} f=f \circ \Phi_{-t}$ on $\mathcal{B}_{\sim}^{0}$ by density and the Lasota-Yorke estimate (Proposition 4.1), bypassing the use of analogues of the spaces $\mathcal{C}^{\beta}\left(T^{-n} \mathcal{W}^{s}\right)$ in [DZ1]. (In view of [DZ1, Lemma 3.7] for the discrete time billiard, one can expect that $\mathcal{B}=\mathcal{B}_{\sim}^{0}$, but this will not be needed.)

We establish relations between our spaces $\mathcal{B}$ and $\mathcal{B}_{w}$ and smooth functions on $\Omega_{0}$.

Lemma 3.9 (Embedding smooth functions). The following continuous injective embeddings hold

$$
\begin{aligned}
& \mathcal{C}^{1}\left(\Omega_{0}\right) \hookrightarrow \mathcal{B}^{0}, \quad \mathcal{C}_{\sim}^{0} \cap \mathcal{C}^{1}\left(\Omega_{0}\right) \hookrightarrow \mathcal{B}_{\sim}^{0} \hookrightarrow \mathcal{B}_{w}, \quad \mathcal{B} \hookrightarrow\left(\mathcal{C}^{\beta}\left(\Omega_{0}\right)\right)^{*}, \\
& \mathcal{B} \hookrightarrow\left(\mathcal{C}^{1}\left(\Omega_{0}\right)\right)^{*}, \quad \text { and } \quad \mathcal{C}_{\sim}^{0} \cap \mathcal{C}^{2}\left(\Omega_{0}\right) \hookrightarrow \mathcal{C}^{1}\left(\Omega_{0}\right) \hookrightarrow \mathcal{B} \hookrightarrow \mathcal{B}_{w} .
\end{aligned}
$$

(The proof uses $1 / q \leq 1, \beta<1 / q$, and $\gamma \leq 1 / 2$.)

Proof. First we show that if $f \in \mathcal{C}^{1}\left(\Omega_{0}\right)$, then $f \in \mathcal{B}^{0}$ and in particular,

$$
\|f\|_{\mathcal{B}} \leq C|f|_{\mathcal{C}^{1}\left(\Omega_{0}\right)}
$$

for a uniform constant $C$. This immediately implies that if $f \in \mathcal{C}^{1}\left(\Omega_{0}\right) \cap \mathcal{C}_{\sim}^{0}$, then $f \in \mathcal{B}_{\sim}^{0}$.

To estimate the neutral component $\|f\|_{0}$, fix $W \in \mathcal{W}^{s}$ and $\psi \in \mathcal{C}^{\alpha}(W)$ with $|\psi|_{\mathcal{C}^{\alpha}} \leq 1$. Then, recalling (2.5) and the notation in (2.1), we have

$$
\left.\int_{W} \partial_{t}\left(f \circ \Phi_{t}\right)\right|_{t=0} \psi d m_{W}=\int_{W} \nabla f \cdot \hat{\eta} \psi d m_{W} \leq|\nabla f \cdot \hat{\eta}|_{\infty}|\psi|_{\infty}|W| \leq L_{0}|f|_{\mathcal{C}^{1}\left(\Omega_{0}\right)} .
$$

To estimate $\|f\|_{s}$, fix $W \in \mathcal{W}^{s}$ and $\psi \in \mathcal{C}^{\beta}(W)$ with $|W|^{1 / q}|\psi|_{\mathcal{C}^{\beta}(W)} \leq 1$. Then

$$
\int_{W} f \psi d m_{W} \leq|f|_{\infty}|\psi|_{\infty}|W| \leq|f|_{\infty} L_{0}^{1-1 / q}
$$

Finally, we estimate $\|f\|_{u}$. Fix $\varepsilon \in(0,1)$ (if $\varepsilon \geq 1$ we may bound the relevant quotient by a constant multiple of the weak norm) and $W_{1}, W_{2} \in \mathcal{W}^{s}$ with $d_{\mathcal{W}^{s}}\left(W_{1}, W_{2}\right) \leq \varepsilon$. For $i=1$, 2 , let $\psi_{i} \in \mathcal{C}^{\alpha}\left(W_{i}\right)$ with $\left|\psi_{i}\right|_{\mathcal{C}^{\alpha}\left(W_{i}\right)} \leq 1$ and $d\left(\psi_{1}, \psi_{2}\right)=0$.

By definition of $d_{\mathcal{W}}$, the trace curves $P^{+}\left(W_{1}\right)$ and $P^{+}\left(W_{2}\right)$ are defined over a common $r$ interval $I$, apart from at most two endpieces which have length no more than $\varepsilon$. Let $U_{1} \subset$ $W_{1}, U_{2} \subset W_{2}$ denote the curves for which $P^{+}\left(U_{1}\right)$ and $P^{+}\left(U_{2}\right)$ are defined as graphs over $I$. Denote by $V_{1}$ and $V_{2}$ the (at most two) pieces which are not defined over $I$. Without loss of generality, we may assume $V_{1} \subset W_{1}$ and $V_{2} \subset W_{2}$. Now,

$$
\begin{aligned}
\int_{W_{1}} f \psi_{1} d m_{W_{1}}-\int_{W_{2}} f \psi_{2} d m_{W_{2}}= & \int_{U_{1}} f \psi_{1} d m_{W_{1}}-\int_{U_{2}} f \psi_{2} d m_{W_{2}} \\
& +\int_{V_{1}} f \psi_{1} d m_{W_{1}}-\int_{V_{2}} f \psi_{2} d m_{W_{2}} .
\end{aligned}
$$

We use the fact that $\left|P^{+}\left(V_{i}\right)\right| \leq \varepsilon$ to bound the estimate over the unmatched pieces,

$$
\left|\int_{V_{i}} f \psi_{i} d m_{W_{i}}\right| \leq\left|V_{i}\right||f|_{\infty} \leq C \varepsilon|f|_{\infty}
$$


where the constant $C$ depends only on the Jacobian of the map from $P^{+}\left(V_{i}\right)$ to $V_{i}$, which is uniformly bounded by the proof of Lemma 3.4.

Next, we estimate the contribution from the matched curves $U_{i}$,

$$
\begin{aligned}
\int_{U_{1}} f \psi_{1} d m_{W_{1}}-\int_{U_{2}} f \psi_{2} d m_{W_{2}} & =\int_{U_{1}}\left(f \psi_{1}-\left(f \cdot \psi_{2}\right) \circ \Theta \cdot J \Theta\right) d m_{W_{1}} \\
& \leq\left|U_{1}\right|\left|f \psi_{1}-\left(f \cdot \psi_{2}\right) \circ \Theta \cdot J \Theta\right|_{C^{0}\left(U_{1}\right)}
\end{aligned}
$$

where $\Theta$ is the map from $U_{1}$ to $U_{2}$ defined via the trace curves: $\Theta=S_{U_{2}} \circ S_{U_{1}}^{-1}$, where $S_{U_{i}}(r)=$ $\Phi_{-t(r)} \circ G_{U_{i}}(r): I \rightarrow U_{i}$ is defined as in Lemma 3.4. Note that since $d\left(\psi_{1}, \psi_{2}\right)=0$, we have $\psi_{2} \circ \Theta=\psi_{1}$ on $U_{1}$. Thus to estimate the sup norm of the difference above, it suffices to estimate $|f-f \circ \Theta|_{\mathcal{C}^{0}\left(U_{1}\right)}$ and $|1-J \Theta|_{\mathcal{C}^{0}\left(U_{1}\right)}$. Note also that $J \Theta$ is bounded by Lemma 3.4, so this factor contributes only a bounded constant to our estimate.

Now since the distance between $P^{+}\left(U_{1}\right)$ and $P^{+}\left(U_{2}\right)$ along vertical segments is at most $\varepsilon$ and since the Jacobians of $S_{U_{2}}$ and $S_{U_{2}}^{-1}$ are uniformly bounded by Lemma 3.4, we have $d(Z, \Theta(Z)) \leq$ $C \varepsilon$, for $Z \in U_{1}$. Since $f \in \mathcal{C}^{1}\left(\Omega_{0}\right)$, we have $|f-f \circ \Theta|_{\mathcal{C}^{0}\left(U_{1}\right)} \leq|f|_{\mathcal{C}^{1}} \varepsilon$.

It remains to estimate $|1-J \Theta|_{\mathcal{C}^{0}\left(U_{1}\right)}$. Using the notation of Lemma 3.4 and denoting by $\tau_{1}$ and $\tau_{2}$ the first collision times starting on $U_{1}$ and $U_{2}$, we have

$$
\begin{aligned}
J \Theta & =\left(J_{P^{+}\left(U_{2}\right)} \Phi_{-\tau_{2}}\right) \circ\left(G_{U_{2}} \circ S_{U_{1}}^{-1}\right) \cdot\left(J G_{U_{2}} \circ S_{U_{1}}^{-1}\right) \cdot\left(J G_{U_{1}}^{-1} \circ \Phi_{\tau_{1}}\right) \cdot\left(J_{U_{1}} \Phi_{\tau_{1}}\right) \\
& =\frac{\left(J_{P^{+}\left(U_{2}\right)} \Phi_{-\tau_{2}}\right) \circ G_{U_{2}} \circ S_{U_{1}}^{-1}}{\left(J_{P^{+}\left(U_{1}\right)} \Phi_{-\tau_{1}}\right) \circ \Phi_{\tau_{1}}} \cdot \frac{J G_{U_{2}} \circ S_{U_{1}}^{-1}}{J G_{U_{1}} \circ S_{U_{1}}^{-1}} .
\end{aligned}
$$

For $Z \in U_{1}$, the points $G_{U_{2}} \circ S_{U_{1}}^{-1}(Z)$ and $\Phi_{\tau_{1}}(Z)$ lie on the same vertical line in $(r, \varphi)$ coordinates; thus the contribution of $\mathcal{K}$ in the analogue of (3.6) vanishes. Hence, adapting (3.6) and the following lines (using $\left|\tau_{1}(Z)-\tau_{2}\left(S_{U_{2}} S_{U_{1}}^{-1}(Z)\right)\right| \leq C \sqrt{\varepsilon}$ ), we have

$$
\sup _{U_{1}}\left|\ln \frac{J_{P^{+}\left(U_{2}\right)} \Phi_{-\tau_{2}}\left(G_{U_{2}} \circ S_{U_{1}}^{-1}\right)}{J_{P^{+}\left(U_{1}\right)} \Phi_{-\tau_{1}}\left(\Phi_{\tau_{1}}\right)}\right| \leq C \varepsilon^{1 / 2},
$$

for some uniform constant $C>0$. Also, by (3.5) and using $\varphi_{1}(r)$ and $\varphi_{2}(r)$ to denote the functions defining $P^{+}\left(U_{1}\right)$ and $P^{+}\left(U_{2}\right)$, we obtain the following bound on $I$ :

$$
\begin{aligned}
\left|\ln \frac{J G_{U_{2}}\left(S_{U_{1}}\right)}{J G_{U_{1}}\left(S_{U_{1}}\right)}\right| & =\frac{1}{2}\left|\ln \left(1+\left(\frac{d \varphi_{2}}{d r}\right)^{2}\right)-\ln \left(1+\left(\frac{d \varphi_{1}}{d r}\right)^{2}\right)\right| \\
& \leq \frac{1}{2+2 \mathcal{K}_{\min }}\left|\left(\frac{d \varphi_{2}}{d r}\right)^{2}-\left(\frac{d \varphi_{1}}{d r}\right)^{2}\right| \leq \frac{\mathcal{K}_{\max }+\tau_{\min }^{-1}}{1+\mathcal{K}_{\min }}\left|\frac{d \varphi_{2}}{d r}-\frac{d \varphi_{1}}{d r}\right| \leq C \varepsilon,
\end{aligned}
$$

by definition of $d_{\mathcal{W}^{s}}\left(W_{1}, W_{2}\right)$. By (3.13), these two estimates imply $|1-J \Theta|_{\mathcal{C}^{0}\left(U_{1}\right)} \leq C \varepsilon^{1 / 2}$.

Putting together the estimates for matched and unmatched pieces and dividing by $\varepsilon^{\gamma}$ yields

$$
\varepsilon^{-\gamma}\left|\int_{W_{1}} f \psi_{1} d m_{W}-\int_{W_{2}} f \psi_{2} d m_{W}\right| \leq C \varepsilon^{1-\gamma}|f|_{\infty}+C \varepsilon^{1 / 2-\gamma}|f|_{\mathcal{C}^{1}\left(\Omega_{0}\right)} .
$$

(Recall that $\gamma<\alpha \leq 1 / 3$.)

The above estimates imply $\|f\|_{\mathcal{B}} \leq C|f|_{\mathcal{C}^{1}(\Omega)}$ for a uniform constant $C$, as claimed. This implies continuity of the embeddings $\mathcal{C}^{1}\left(\Omega_{0}\right) \hookrightarrow \mathcal{B}^{0}$ and $\mathcal{C}_{\sim}^{0} \cap \mathcal{C}^{1}\left(\Omega_{0}\right) \hookrightarrow \mathcal{B}_{\sim}^{0}$. Injectivity is obvious.

As for the embedding $\mathcal{B}_{\sim}^{0} \hookrightarrow \mathcal{B}_{w}$, continuity follows from the fact that $|\cdot|_{w} \leq\|\cdot\|_{s}$, while injectivity follows from the definition of the spaces $\mathcal{C}^{\alpha}(W)$ and $\mathcal{C}^{\beta}(W)$ as the closures of $\mathcal{C}^{1}(W)$ in the respective Hölder norms. Similarly, the embedding $\mathcal{B} \hookrightarrow \mathcal{B}_{w}$ is continuous and injective by the same observations. The embedding $\mathcal{C}^{1}\left(\Omega_{0}\right) \hookrightarrow \mathcal{B}$ is the content of Lemma 7.5 , while injectivity is 
again obvious. Finally, continuity of the embeddings $\mathcal{B} \hookrightarrow\left(\mathcal{C}^{\beta}\left(\Omega_{0}\right)\right)^{*}$ and $\mathcal{B} \hookrightarrow\left(\mathcal{C}^{1}\left(\Omega_{0}\right)\right)^{*}$ follow from Lemma 2.11, while injectivity is proved in Lemma 7.1, using $\beta<1 / q$.

We close this section with the following compactness result.

Lemma 3.10 (Compact embedding). The unit ball of $\mathcal{B}$ is compactly embedded in $\mathcal{B}_{w}$.

(Recall that $\beta<\alpha$. Like in [DZ1, the proof also uses $c_{u}>0$.)

Proof. Recalling (3.11), it suffices to show that the unit ball of $\mathcal{B}_{\sim}^{0}$ is compactly embedded in $\mathcal{B}_{w}$. The general strategy of our proof will be to create, for each $\varepsilon>0$, an $\varepsilon$-covering of $\Omega$ by finitely many curves $W_{i}$ in $\mathcal{W}^{s}$ and an $\varepsilon$-covering of $C^{\alpha}\left(W_{i}\right)$ by finitely many functions $\psi_{j}$ so that the weak norm of any $f \in \mathcal{B}$ with $\|f\|_{\mathcal{B}} \leq 1$ can be uniformly approximated by measuring it against the finitely many functionals defined by $\int_{W_{i}} f \psi_{j} d m_{W}$.

We may assume without restricting generality that there exists $\ell$ so that $P^{+}(W) \in \Gamma_{\ell} \times$ $[-\pi / 2, \pi / 2]$, i.e, we argue one scatterer-component $\Omega^{(\ell)}:=\left(P^{+}\right)^{-1}\left(\Gamma_{\ell} \times[-\pi / 2, \pi / 2]\right)$ at a time. Let $0<\varepsilon \leq 1$ be fixed. We split $\Omega^{(\ell)}$ into two parts, the good set

$$
A(\ell, \varepsilon)=\left(P^{+}\right)^{-1}(\{-\pi / 2+\varepsilon \leq \varphi \leq \pi / 2-\varepsilon\}) \cap \Omega^{(\ell)},
$$

and the bad set $B(\ell, \varepsilon)=\Omega^{(\ell)} \backslash A(\ell, \varepsilon)$ (that is, $\varepsilon$-close to tangential collisions). The image under $P^{+}$of stable curves are graphs of decreasing functions $\varphi_{W}$ of absolute value of the slope larger than $\mathcal{K}_{\min }>0$ and with uniformly bounded second derivatives. If $W \subset B(\ell, \varepsilon)$, then since $P^{+}(W)$ is transversal to the boundary of the scatterer, it has length $O(\varepsilon)$. Since the expansion from $P^{+}(W)$ to $W$ is of order 1 for a stable curve by Lemma 3.4, there exists $C=C(Q)$ so that any admissible curve $W \subset B(\ell, \varepsilon)$ has length at most $C \varepsilon$.

Let $f \in \mathcal{C}_{\sim}^{0}$ with $\|f\|_{\mathcal{B}} \leq 1$. First, we estimate the weak norm of $f$ on curves $W \in B(\ell, \varepsilon)$. If $|\psi|_{\mathcal{C}^{\alpha}(W)} \leq 1$, then, using the bound $C \varepsilon$ on the length of $W$,

$$
\int_{W} f \psi d m_{W} \leq\|f\|_{s}|W|^{1 / q}|\psi|_{\mathcal{C}^{\beta}(W)} \leq C^{1 / q} \varepsilon^{1 / q}\|f\|_{s} .
$$

In order to study curves $W \in A(\ell, \varepsilon)$, we need some preparations.

Letting $\delta_{1}\left(L_{0}\right)$ and $B_{1}\left(B_{0}\right)$ (uniform in $\varepsilon$ ) be as in the proof of Lemma 3.8, for any map-stable curve $V \in \mathcal{W}^{s}(T) \cap P^{+}(A(\ell, \varepsilon))$ of length $\leq \delta_{1}$ and curvature bounded by $B_{1}$, the surface

$$
V^{0}=\left\{\Phi_{-t}\left(r, \varphi_{V}(r)\right) \mid r \in I_{V}, 0 \leq t<\tau_{-}\left(r, \varphi_{V}(r)\right)\right\}
$$

(where $V$ is the graph of $\varphi_{V}$ defined on $I_{V}$, and $\tau_{-}(z)$, for $z \in \mathcal{M}$, is the smallest $t>0$ so that $\left.\Phi_{-t}(z) \in \mathcal{M}\right)$ is foliated by admissible flow-stable curves $W$ so that $P^{+}(W) \subset V$. Indeed, if $Z \in V^{0}$, there is a unique curve $W=W(Z, V) \subset V^{0}$ containing $Z$ and so that $W$ is everywhere perpendicular to the flow. We take $W$ maximal with these properties, noting that the endpoints of $W$ either (1) project to endpoints of $V$; (2) lie on a scatterer, i.e., are of the form

$$
\Phi_{-\tau_{-}\left(r, \varphi_{V}(r)\right)}\left(r, \varphi_{V}(r)\right) \text {, }
$$

or: (3) undergo a grazing collision under the flow in forward time. Such a curve $W$ is $\mathcal{C}^{2}$ and flowstable by construction, it satisfies the admissibility requirements for $L_{0}$ and $B_{0}$, if $\delta_{1}$ and $B_{1}$ are small enough. (Note that for any flow stable curves $W_{1} \neq W_{2} \subset V^{0}$ we have $d_{\mathcal{W}^{s}}\left(W_{1}, W_{2}\right)=\infty$.) All such curves $W$ are constant-time flow translates of one another, except near collisions, where some shortening of the curve, due to the variable collision times, may occur. Conversely, if $W \subset A(\ell, \varepsilon)$ is an admissible flow curve for $L_{0}$ and $B$, then $W$ belongs to the surface $\left(P^{+}(W)\right)^{0}$, which is foliated by flow-stable curves, admissible for $\delta_{2}\left(L_{0}\right)$ and $B_{2}\left(B_{0}\right)$, uniformly in $\varepsilon$.

On a fixed $r$-interval $I$, the set of functions $\left\{\varphi_{V}\right\}$ for map-stable curves $V \in \mathcal{W}^{s}(T)$, defined on $I$ is uniformly bounded in $\mathcal{C}^{2}$ norm and therefore compact in the $\mathcal{C}^{1}$ norm. There exists 
a finite set of admissible map-stable curves $\left\{V_{i}^{\prime}\right\}_{i=1}^{I_{\varepsilon}^{\prime}} \subset P^{+}(A(\ell, \varepsilon))$ so that for any flow-stable admissible curve $W \subset A(\ell, \varepsilon)$ there exists $i$ with $\tilde{d}_{\mathcal{W}^{s}}\left(V_{i}^{\prime}, P^{+}(W)\right)<\varepsilon$. Therefore, since the stable and unstable cones $C^{s}$ and $C^{u}$ are uniformly transverse, and are both orthogonal to the flow direction, we may choose a finite set of admissible map-stable curves $\left\{V_{i}\right\}_{i=1}^{I_{\varepsilon}} \subset P^{+}(A(\ell, \varepsilon))$ so that for any flow-stable admissible curve $W \subset A(\ell, \varepsilon)$, there exist:

(i) an index $i_{W}$ so that $\tilde{d}_{\mathcal{W}^{s}}\left(P^{+}(W), V_{i_{W}}\right)<\varepsilon$, and a flow-unstable curve $W^{u}$ with $W^{u} \cap W \neq$ $\emptyset$ and $W^{u} \cap V_{i_{W}}^{0} \neq \emptyset$;

(ii) a flow-stable curve $W^{\prime} \subset V_{i_{W}}^{0}$ with $W^{u} \cap V_{i_{W}}^{0} \in W^{\prime}$ and $d_{\mathcal{W}^{s}}\left(W, W^{\prime}\right)<C \sqrt{\varepsilon}$, for some uniform $C>0$.

Item (i) above is obvious, as is the existence of $W^{\prime}$ with $W^{u} \cap V_{i_{W}}^{0} \in W^{\prime} \subset V_{i_{W}}^{0}$ in item (ii). To prove the bound on the distance between $W$ and $W^{\prime}$ in (ii), first note that the tangent vectors to $P^{+}\left(W^{u}\right)$ lie in the map-unstable cone by forward invariance of the unstable cones, and that $P^{+}\left(W^{u}\right)$ intersects both $V_{i_{W}}$ and $P^{+}(W)$. By choice of the index $i_{W}$, the length of the segment in $P^{+}\left(W^{u}\right)$ connecting $P^{+}(W)$ to $V_{i_{W}}$ is at most $C \varepsilon$. Since $W^{u}$ intersects both $W$ and $W^{\prime}$ and the length of unstable curves is expanded going forward, it follows that the length of the segment in $W^{u}$ connecting $W$ and $W^{\prime}$ is at most $C \varepsilon$ as well. Finally, the fact that $P^{+}\left(W^{\prime}\right) \subset V_{i_{W}}$ and $\tilde{d}_{\mathcal{W}^{s}}\left(P^{+}(W), V_{i_{W}}\right)<\varepsilon$, together with the 1/2-Hölder continuity of $\tau$, implies that the distance $\tilde{d}_{\mathcal{W}^{s}}\left(P^{+}(W), P^{+}\left(W^{\prime}\right)\right)$ (which is due only to the endpoints-discrepancy) satisfies $\tilde{d}_{\mathcal{W}^{s}}\left(P^{+}(W), P^{+}\left(W^{\prime}\right)\right)<C \sqrt{\varepsilon}$ (recalling (3.15) ) as required 22

We next decompose each $V_{i}$ corresponding to collision times to handle the shortening of the curve due to the variable collision times mentioned above. We let $M_{\varepsilon}=\left[\epsilon^{-1}\right]$,

$$
\tau_{i, \min }=\min \tau_{-}\left(r, \varphi_{V}(r)\right), \tau_{i, m}=\tau_{i, \min }+m M_{\varepsilon}^{-1}\left(\max \tau_{-}\left(r, \varphi_{V}(r)\right)-\tau_{i, \min }\right), m=0, \ldots, M_{\varepsilon} .
$$

Note that $\tau_{i, \min }$ is the first collision time when flowing $V_{i}$ backwards. It is not enough to flow $V_{i}$ back until this first collision: If $W$ is a stable curve with $P^{+}(W) \varepsilon$-close to $V_{i}$, the curve $W$ may be in fact very far from any of the curves spanned by $V_{i}$ up to time $-\tau_{i, \text { min }}$ (for example, if a little piece of $V_{i}$ hits a nearby scatterer under the backwards flow, but most of the rest of $V_{i}$ continues without collision for some time). Still, there is a subcurve $V_{i}^{\prime}$ of $V_{i}$, differing in length 23 by no more than $\varepsilon$ from $V_{i}$, such that $V_{i}^{\prime}$ does not hit this close scatterer, and the backward translates of $V_{i}^{\prime}$ contain a stable curve which is close to $W$ and connected with $W$ by an unstable curve. By the triangle inequality, $V_{i}^{\prime}$ and $P^{+}(W)$ differ by no more than $2 \varepsilon$.)

Let us now formalise the above discussion: We construct nested curves by setting $V_{i, 0}=V_{i}=$ $\left\{V_{i} \cap \tau_{-}^{-1}\left[\tau_{i, 0}, \infty\right)\right\}$ and, for $m=0, \ldots, M_{\varepsilon}$,

$$
V_{i, m}=\left\{V_{i} \cap \tau_{-}^{-1}\left[\tau_{i, m}, \infty\right)\right\} \subset V_{i, m-1} .
$$

(If any of the $V_{i, m}$ is disconnected, we subdivide it into its finitely many connected components, without making this explicit in the notation for the sake of conciseness.) Finally, in each $V_{i, m}$ we choose a point $v_{i, m}$ so that $\tau_{-}\left(v_{i, m}\right)=\tau_{i, m}$.

Then, there exists a nonnegative real number $\Theta_{\varepsilon}=O(\varepsilon)$, and a finite integer $N_{\varepsilon}=O\left(\Theta_{\varepsilon}^{-1}\right)$, such that 24 the set of flow-stable curves

$$
\begin{aligned}
& \left\{W\left(\Phi_{-\theta}\left(\Phi_{-\tau_{-}\left(v_{i, m}\right) \frac{n}{N_{\varepsilon}}}\left(v_{i, m}\right)\right), V_{i, m}\right),\right. \\
& \left.\quad i=1, \ldots, I_{\varepsilon}, m=0, \ldots, M_{\varepsilon}, n=0, \ldots, N_{\varepsilon}-1, \theta \in\left[0, \Theta_{\varepsilon}\right]\right\}
\end{aligned}
$$

\footnotetext{
22 The bound $<\sqrt{\epsilon}$ can perhaps be improved to $<C \epsilon$ by making a special choice of $W^{u}$.

23 By construction of the family $V_{i}$.

${ }^{24}$ We do not claim that $I_{\varepsilon}, N_{\varepsilon}$, or $\Theta_{\varepsilon}^{-1}$ are bounded uniformly in $\varepsilon$.
} 
forms an $\sqrt{\varepsilon}$-covering of the admissible flow-stable curves $\left.\mathcal{W}^{s}\right|_{(A(\ell, \varepsilon))}$ in the distance $d_{\mathcal{W}^{s}}$, which follows by applying items (i) and (ii) explained above.

By definition, the uniquely defined $\mathcal{C}^{1}$ functions $\tilde{t}_{i, m, n, \theta}$ on $V_{i, m}$ so that

$\widetilde{W}_{i, m, n, \theta}:=W\left(\Phi_{-\theta} \Phi_{-\tau_{-}\left(v_{i, m}\right) \frac{n}{N_{\varepsilon}}}\left(v_{i, m}\right), V_{i, m}\right)$ is given by $\Phi_{-\tilde{t}_{i, m, n, \theta}(r)}\left(r, \varphi_{V_{i}}(r)\right)=: \tilde{S}_{i, m, n, \theta}(r)$

satisfy $\tilde{t}_{i, m, n, \theta}-\tilde{t}_{i, m, n, 0} \equiv \theta$.

Set $t_{i, m, n}=\tilde{t}_{i, m, n, 0}$, so that $W_{i, m, n}:=W\left(\Phi_{-\tau_{-}\left(v_{i, m}\right) \frac{n}{N_{\varepsilon}}}\left(v_{i, m}\right), V_{i, m}\right)$ is given by

$$
\Phi_{-t_{i, m, n}(r)}\left(r, \varphi_{V_{i, m}}(r)\right)=: S_{i, m, n}(r) .
$$

Let $\left|\Gamma_{\ell}\right|$ denote the arclength of $\Gamma_{\ell}$, and define $\mathbb{S}_{\ell}^{1}$ to be the circle of length $\left|\Gamma_{\ell}\right|$. Since any ball of finite radius in the $\mathcal{C}^{\alpha}$ norm is compactly embedded in $\mathcal{C}^{\beta}$, we may choose finitely many functions $\bar{\psi}_{j} \in \mathcal{C}^{\alpha}$ such that $\left\{\bar{\psi}_{j}\right\}_{j=1}^{J_{\varepsilon}}$ forms an $\varepsilon$-covering in the $\mathcal{C}^{\beta}\left(\mathbb{S}_{\ell}^{1}\right)$-norm of the ball of radius $C C_{0}$ in $\mathcal{C}^{\alpha}\left(\mathbb{S}_{\ell}^{1}\right)$, with $C_{0}$ the constant depending on $\mathcal{K}_{\max }, \tau_{\max }$, and $1 / \tau_{\min }$ from Lemma 3.4.

This ends the announced preparations for the case $W \subset A(\ell, \varepsilon)$.

From now on, fix $W=\left.S_{W}\left(I_{W}\right) \in \mathcal{W}^{s}\right|_{A(\ell, \varepsilon)}$, and $\psi \in \mathcal{C}^{\alpha}(W)$ with $|\psi|_{\mathcal{C}^{\alpha}(W)} \leq 1$. We view $I_{W}$ as a subset of $\mathbb{S}_{\ell}^{1}$. Let $\bar{\psi}=\psi \circ S_{W}$ be the push down of $\psi$ to $I_{W}$. By definition, $S_{W}(r)=\Phi_{-t_{W}(r)} G_{W}(r)$ with $G_{W}$ the graph of the function $\varphi_{W}$. By the proof of Lemma 3.4. the Jacobian of $S_{W}$ is bounded by $C_{0}$, and we have

$$
|\bar{\psi}|_{\mathcal{C}^{\alpha}\left(I_{W}\right)} \leq C C_{0} .
$$

Choose $\bar{\psi}_{j} \in \mathcal{C}^{\alpha}\left(\mathbb{S}_{\ell}^{1}\right)$ so that $\left|\bar{\psi}-\bar{\psi}_{j}\right|_{\mathcal{C}^{\beta}\left(I_{W}\right)} \leq \varepsilon$.

Take $i, m, n$, and $\theta$ so that $d_{\mathcal{W}^{s}}\left(W, \widetilde{W}_{i, m, n, \theta}\right)<\sqrt{\varepsilon}$. Define $\psi_{j, i, m, n}=\bar{\psi}_{j} \circ S_{i, m, n}^{-1}$ to be the lift of $\bar{\psi}_{j}$ to $W_{i, m, n}$. Note that $\left|\psi_{j, i, m, n}\right|_{\mathcal{C}^{\alpha}\left(W_{i, m, n}\right)} \leq 2 C C_{0}$, again by Lemma 3.3

Then, normalizing $\psi$ and $\psi_{j, i, m, n}$ by $2 C C_{0}$, and letting $J_{W, i, m, n, \theta}=J_{W_{i, m, n}} \Phi_{-\theta}$ be the Jacobian of the map $\tilde{S}_{i, m, n, \theta} \circ S_{i, m, n}^{-1}=\Phi_{-\theta}$ from $W_{i, m, n}$ to $\widetilde{W}_{i, m, n, \theta}$, we decompose

$$
\begin{aligned}
& \left|\int_{W} f \psi d m_{W}-\int_{W_{i, m, n}} f \psi_{j, i, m, n} d m_{W_{i, m, n}}\right| \\
& \leq\left|\int_{W} f \psi d m_{W}-\int_{\widetilde{W}_{i, m, n, \theta}} f\left(\bar{\psi}_{j} \circ \tilde{S}_{i, m, n, \theta}^{-1}\right) d m_{\widetilde{W}_{i, m, n, \theta}}\right| \\
& \quad+\left|\int_{W_{i, m, n}}\left[\left(f \circ \tilde{S}_{i, m, n, \theta} \circ S_{i, m, n}^{-1}\right) \psi_{j, i, m, n} J_{W, i, m, n, \theta}-f \psi_{j, i, m, n}\right] d m_{W_{i, m, n}}\right|,
\end{aligned}
$$

where $\left|J_{W, i, m, n, \theta}-1\right|_{C^{\beta}\left(W_{i, m, n}\right)} \leq C \varepsilon$, so that we can use the triangle inequality and $\|f\|_{s}$ to eliminate $J_{W, i, m, n, \theta}$ in the last term of (3.16). Next, recalling the group property (2.11), we estimate the remaining difference in the last term of (3.16),

$$
\begin{aligned}
\int_{W_{i, m, n}} & \left(f \circ \Phi_{-\theta}-f\right) \psi_{j, i, m, m} d m_{W_{i, m, n}}=\int_{W_{i, m, n}} \int_{0}^{\theta} \partial_{s}\left(f \circ \Phi_{-s}\right) \psi_{j, i, m, n} d s d m_{W_{i, m, n}} \\
& =\left.\int_{0}^{\theta} \int_{W_{i, m, n}} \partial_{r}\left(f \circ \Phi_{r}\right)\right|_{r=0} \circ \Phi_{-s} \psi_{j, i, m, n} d m_{W_{i, m, n}} d s \\
& =\left.\int_{0}^{\theta} \int_{\Phi_{-s}\left(W_{i, m, n}\right)} \partial_{r}\left(f \circ \Phi_{r}\right)\right|_{r=0} \psi_{j, i, m, n} \circ \Phi_{s} J_{\Phi_{-s}\left(W_{i, m, n}\right)} \Phi_{s} \\
& \leq \theta\|f\|_{0}\left|\psi_{j, i, m, n} \circ \Phi_{s}\right|_{\mathcal{C}^{\alpha}\left(\Phi_{s}\left(W_{i, m, n}\right)\right)}\left|J_{\Phi_{-s}\left(W_{i, m, n}\right)} \Phi_{s}\right|_{\mathcal{C}^{\alpha}\left(\Phi_{s}\left(W_{i, m, n}\right)\right)} \leq C \varepsilon\|f\|_{0},
\end{aligned}
$$


where in the last line we have used the smoothness of the flow between collisions to bound both the Hölder norm of the test function and the Jacobian of the change of variables.

Finally, for the first term on the right-hand side of (3.16), we again use the triangle inequality and $\|f\|_{s}$ to replace $\bar{\psi}_{j} \circ \tilde{S}_{i, m, n, \theta}^{-1}$ by a test function $\psi_{i, j, m, n, \theta}$ with $d\left(\psi, \psi_{i, j, m, n, \theta}\right)=0$ and use the unstable norm of $f$ to bound the remaining difference of integrals. Putting these estimates together yields,

$$
\left|\int_{W} f \psi d m_{W}-\int_{W_{i, m, n}} f \psi_{j, i, m, n} d m_{W_{i, m, n}}\right| \leq\left(\varepsilon^{\gamma / 2}\|f\|_{u}+\varepsilon\|f\|_{s}+\varepsilon\|f\|_{0}\right) 2 C C_{0} .
$$

Recalling that $2 \gamma<1$, we have proved that for each $0<\varepsilon \leq 1$, there exist finitely many bounded linear functionals $\ell_{i, j, m, n}$ with $\ell_{i, j, m, n}(f)=\int_{W_{i, m, n}} f \psi_{j, i, m, n} d m_{W_{i, m, n}}$ such that

$$
\begin{aligned}
|f|_{w} & \leq \max _{i \leq I_{\varepsilon}, m \leq M_{\varepsilon}, j \leq J_{\varepsilon}, n \leq N_{\varepsilon}-1} \ell_{i, j, m, n}(f)+2 C C_{0}\left(\left(\varepsilon^{1 / q}+\varepsilon\right)\|f\|_{s}+\varepsilon^{\gamma / 2}\|f\|_{u}+\varepsilon\|f\|_{0}\right) \\
& \leq \max \ell_{i, j, m, n}(f)+C^{\prime} c_{u}^{-1} \varepsilon^{\gamma / 2}\|f\|_{\mathcal{B}} .
\end{aligned}
$$

Since $c_{u}>0$, this implies the required compactness.

\section{LASOTA-YORKE-TYPE BOUNDS AND STRONG CONTINUITY FOR THE SEMI-GROUP $\mathcal{L}_{t}$}

The transfer operator for the flow is defined on $25 \mathcal{C}_{\sim}^{0}($ recall $(2.9))$ by $\mathcal{L}_{t} f=f \circ \Phi_{-t}$ for $t \geq 0$. (In particular, $\mathcal{L}_{0}$ is the identity on $\mathcal{C}_{\sim}^{0}$.) Letting $m$ denote Riemannian volume on $\Omega_{0}$, we also have $\mathcal{L}_{t} f=f \circ \Phi_{-t}$ for any $f \in L^{1}\left(\Omega_{0}, m\right)$, since $\Phi_{t}$ preserves $m$. The Banach space $\mathcal{B}$ is not contained in $\mathcal{C}_{\sim}^{0}$ or $L^{1}(m)$. However, we have that $\mathcal{C}_{\sim}^{0} \cap \mathcal{B}_{\sim}^{0}$ is dense in the auxiliary space $\mathcal{B}_{\sim}^{0}$ by definition (3.11). In this section, we will prove the following proposition (recall $\mathcal{B}^{0}$ from (3.10), and the constants $\Lambda=\Lambda_{0}^{1 / \tau_{\max }}>1$ from (1.2) and $\lambda \in\left(\Lambda_{0}^{-1 / \tau_{\max }}, 1\right)$ from the growth Lemma 3.8).

Proposition 4.1. Recall that $\beta<\alpha$ and $\gamma \leq \min \{\alpha-\beta, 1 / q\}$. There exists $C>0$ such that for all $f \in \mathcal{C}^{0} \cap \mathcal{B}^{0}$ and $t>0$,

$$
\begin{aligned}
\left|\mathcal{L}_{t} f\right|_{w} & \leq C|f|_{w}, \\
\left\|\mathcal{L}_{t} f\right\|_{s} & \leq C\left(\Lambda^{-\beta t}+\lambda^{(1-1 / q) t}\right)\|f\|_{s}+C L_{0}^{-1 / q}|f|_{w}, \\
\left\|\mathcal{L}_{t} f\right\|_{0} & \leq C\|f\|_{0} \\
\left\|\mathcal{L}_{t} f\right\|_{u} & \leq C t^{\gamma} \Lambda^{-\gamma t}\|f\|_{u}+C\|f\|_{0}+C\|f\|_{s} .
\end{aligned}
$$

If we assume in addition $\beta \leq 1-1 / q$, and if we allow $C$ to depend on $L_{0}$, then (4.2) implies

$$
\left\|\mathcal{L}_{t} f\right\|_{s} \leq C \Lambda^{-\beta t}\|f\|_{s}+C|f|_{w} .
$$

Recall that $\|\cdot\|_{u}$ will appear with a (small) factor $c_{u}$ to be introduced later in the norm $\|\cdot\|_{\mathcal{B}}$. Even with this weighting the above bounds (just as in [L2, BL]) are not honest Lasota-Yorke bounds because of (4.3), which is neither a contracted term nor compact. Integration with respect to time in the resolvent $\mathcal{R}(z)$ will yield the true Lasota-Yorke bounds of Proposition 5.1.

It follows from the above proposition that for any $f \in \mathcal{C}_{\sim}^{0} \cap \mathcal{B}_{\sim}^{0} \subset \mathcal{C}^{0} \cap \mathcal{B}^{0}$ and any $t>0$, the image $\mathcal{L}_{t} f$, which is defined as an element of $\mathcal{C}_{\sim}^{0}$, still belongs to $\mathcal{B}_{\sim}^{0}$ and satisfies $\left\|\mathcal{L}_{t} f\right\|_{\mathcal{B}} \leq C\|f\|_{\mathcal{B}}$. Since $\mathcal{C}_{\sim}^{0} \cap \mathcal{B}_{\sim}^{0}$ is dense in $\mathcal{B}_{\sim}^{0}$ the operator $\mathcal{L}_{t}$ can be extended to a continuous operator on $\mathcal{B}_{\sim}^{0}$ for any $t \geq 0$, and indeed Proposition 4.1 holds for all $f \in \mathcal{B}_{\sim}^{0}$. Since $\mathcal{B} \subset \mathcal{B}_{\sim}^{0}$, we get, noticing that the set in (2.10) is $\mathcal{L}_{t}$-invariant for all $t$ :

\footnotetext{
${ }^{25}$ Defining the transfer operator on $L^{\infty}$ leads to problems since an element of $L^{\infty}$ is not in general well-defined on a stable curve $W$.
} 
Corollary 4.2 (Continuity and bounds on $\mathcal{B})$. The operator $\mathcal{L}_{t}$ is continuous on $\mathcal{B}$ for any $t \geq 0$, and the bounds in Proposition 4.1 hold for all $f \in \mathcal{B}$.

The family of bounded operators $\mathcal{L}_{t}$ on $\mathcal{B}$ satisfy:

(i) $\mathcal{L}_{0}$ is the identity on $\mathcal{B}_{\sim}^{0}$ and thus on $\mathcal{B}$;

(ii) For any $0 \leq t, s<\infty$ we have $\mathcal{L}_{t} \circ \mathcal{L}_{s}=\mathcal{L}_{s+t}$.

In Lemma 4.6 (stated and proved Subsection 4.3), we will show that for any $f \in \mathcal{B}$

$$
\lim _{t \downarrow 0} \mathcal{L}_{t}(f)=f,
$$

with convergence in $\mathcal{B}$. This implies the third condition required for a one-parameter semi-group of bounded operators (see [Da, §6, p. 152]), that

(iii) the map $(t, f) \mapsto \mathcal{L}_{t}(f)$ from $[0, \infty) \times \mathcal{B}$ to $\mathcal{B}$ is jointly continuous.

Note also that $\mathcal{L}_{t}$ is bounded on $\mathcal{B}_{w}$ (same argument as above). In fact, Lemma 4.7 in Subsection 4.3 will show that $\mathcal{L}_{t}$ is Lipschitz when viewed as an operator from $\mathcal{B}$ to $\mathcal{B}_{w}$.

In Subsections 4.1 and 4.2, we prove Proposition 4.1. Subsection 4.3 is devoted to Lemmas 4.6 and 4.7. We shall use several times without mention the key observation (recall (2.5)) that a stable curve $W \in \mathcal{W}^{s}$, and more generally any $W_{i} \in \mathcal{G}_{t}(W)$ for $t>0$, may intersect $\partial \Omega_{0}$ in at most two points.

4.1. Weak stable, strong stable, and neutral norm estimates for $\mathcal{L}_{t}$. We start with (4.1). Let $f \in \mathcal{C}^{0} \cap \mathcal{B}^{0}, W \in \mathcal{W}^{s}$ and $\psi \in \mathcal{C}^{\alpha}(W)$ such that $|\psi|_{\mathcal{C}^{\alpha}(W)} \leq 1$. For $t>0$, we write,

$$
\int_{W} \mathcal{L}_{t} f \psi d m_{W}=\sum_{W_{i} \in \mathcal{G}_{t}(W)} \int_{W_{i}} f J_{W_{i}} \Phi_{t} \psi \circ \Phi_{t} d m_{W} \leq \sum_{W_{i} \in \mathcal{G}_{t}(W)}|f|_{w}\left|J_{W_{i}} \Phi_{t}\right|_{\mathcal{C}^{\alpha}\left(W_{i}\right)}\left|\psi \circ \Phi_{t}\right|_{\mathcal{C}^{\alpha}\left(W_{i}\right)},
$$

where we have used the definition of the weak norm on each $W_{i}$. Since $\alpha \leq 1 / 3$, the distortion bounds given by Lemma 3.5 imply that

$$
\left|J_{W_{i}} \Phi_{t}\right|_{\mathcal{C}^{\alpha}\left(W_{i}\right)} \leq C_{d}\left|J_{W_{i}} \Phi_{t}\right|_{L^{\infty}\left(W_{i}\right)} .
$$

By Lemma 3.6, we have $\left|\psi \circ \Phi_{t}\right|_{\mathcal{C}^{\alpha}\left(W_{i}\right)} \leq C_{1}|\psi|_{\mathcal{C}^{\alpha}(W)}$. Using these estimates in equation (4.6), we obtain

$$
\int_{W} \mathcal{L}_{t} f \psi d m_{W} \leq C|f|_{w} \sum_{W_{i} \in \mathcal{G}_{t}(W)}\left|J_{W_{i}} \Phi_{t}\right|_{L^{\infty}\left(W_{i}\right)} \leq C|f|_{w}
$$

where in the last inequality we have used Lemma 3.8(b) with $1 / q_{0}=0$. Taking the supremum over all $W \in \mathcal{W}^{s}$ and $\psi \in \mathcal{C}^{\alpha}(W)$ with $|\psi|_{\mathcal{C}^{\alpha}(W)} \leq 1$ yields (4.1).

We next prove (4.2). Let $f \in \mathcal{C}^{0} \cap \mathcal{B}^{0}, t>0, W \in \mathcal{W}^{s}$, and let $\left\{W_{i}^{t}\right\}$ denote the elements of $\mathcal{G}_{t}(W)$. For $\psi \in \mathcal{C}^{\beta}(W)$, with $|W|^{1 / q}|\psi|_{\mathcal{C}^{\beta}(W)} \leq 1$, define $\bar{\psi}_{i}=\left|W_{i}^{t}\right|^{-1} \int_{W_{i}^{t}} \psi \circ \Phi_{t} d m_{W}$. Using equation (4.6), we write

$$
\int_{W} \mathcal{L}_{t} f \psi d m_{W}=\sum_{i} \int_{W_{i}^{t}} f J_{W_{i}^{t}} \Phi_{t}\left(\psi \circ \Phi_{t}-\bar{\psi}_{i}\right) d m_{W}+\bar{\psi}_{i} \int_{W_{i}^{t}} f J_{W_{i}^{t}} \Phi_{t} d m_{W}
$$

To bound the first term of (4.9), we first estimate $\left|\psi \circ \Phi_{t}-\bar{\psi}_{i}\right|_{\mathcal{C}^{\beta}\left(W_{i}^{t}\right)}$. Since $\bar{\psi}_{i}$ is constant on $W_{i}^{t}$, we have $\mathcal{C}_{W_{i}^{t}}^{\beta}\left(\psi \circ \Phi_{t}-\bar{\psi}_{i}\right) \leq C_{1} \Lambda^{-\beta t} \mathcal{C}_{W}^{\beta}(\psi)$ by Lemma 3.6. To estimate the $L^{\infty}$ norm, note that $\bar{\psi}_{i}=\psi \circ \Phi_{t}\left(Z_{i}\right)$ for some $Z_{i} \in W_{i}^{t}$. Thus for each $Z \in W_{i}^{t}$,

$$
\left|\psi \circ \Phi_{t}(Z)-\bar{\psi}_{i}\right|=\left|\psi \circ \Phi_{t}(Z)-\psi \circ \Phi_{t}\left(Z_{i}\right)\right| \leq \mathcal{C}_{W_{i}^{t}}^{\beta}\left(\psi \circ \Phi_{t}\right)\left|W_{i}^{t}\right|^{\beta} \leq C \mathcal{C}_{W}^{\beta}(\psi) \Lambda^{-\beta t} .
$$


These estimates together with the fact that $|W|^{1 / q}|\psi|_{\mathcal{C}^{\beta}(W)} \leq 1$, imply

$$
\left|\psi \circ \Phi_{t}-\bar{\psi}_{i}\right|_{\mathcal{C}^{\beta}\left(W_{i}^{t}\right)} \leq C \Lambda^{-\beta t}|\psi|_{\mathcal{C}^{\beta}(W)} \leq C \Lambda^{-\beta t}|W|^{-1 / q} .
$$

We apply (4.10), the distortion estimate (4.7) and the definition of the strong stable norm to the first term on the right-hand side of (4.9),

$$
\begin{aligned}
\sum_{i} \int_{W_{i}^{t}} f J_{W_{i}^{t}} \Phi_{t}\left(\psi \circ \Phi_{t}-\bar{\psi}_{i}\right) d m_{W} & \leq C \sum_{i}\|f\|_{s} \frac{\left|W_{i}^{t}\right|^{1 / q}}{|W|^{1 / q}}\left|J_{W_{i}^{t}} \Phi_{t}\right|_{L^{\infty}\left(W_{i}^{t}\right)} \Lambda^{-\beta t} \\
& \leq C^{\prime} \Lambda^{-\beta t}\|f\|_{s},
\end{aligned}
$$

where in the second line we have used Lemma 3.8(b) with $q_{0}=q$.

For the second term on the right-hand side of (4.9), we use the fact that $\left|\bar{\psi}_{i}\right| \leq|W|^{-1 / q}$ since $|W|^{1 / q}|\psi|_{\mathcal{C}^{\beta}(W)} \leq 1$. We group the curves $W_{i}^{t} \in \mathcal{G}_{t}(W)$ according to most recent long ancestors, as in the proof of Lemma 3.8. As before, $t_{k}=t-k \tau_{\min }, k=0, \ldots,\left\lfloor t / \tau_{\min }\right\rfloor$, and each $W_{i}^{t}$ belongs either to $A_{t}\left(W_{j}^{t_{k}}\right)$ for some $W_{j}^{t_{k}} \in \mathcal{G}_{t_{k}}(W)$ or $W_{i}^{t} \in \mathcal{I}_{t}(W)$.

Using this grouping, we estimate the second term in (4.9) by

$$
\begin{aligned}
\sum_{i}|W|^{-1 / q} \int_{W_{i}^{t}} f J_{W_{i}^{t}} \Phi_{t} d m_{W}= & \sum_{k=0}^{\left\lfloor t / \tau_{\min }\right\rfloor} \sum_{j \in L_{k}(W, t)} \sum_{i \in A_{t}\left(W_{j}^{t}\right)}|W|^{-1 / q} \int_{W_{i}^{t}} f J_{W_{i}^{t}} \Phi_{t} d m_{W} \\
& +\sum_{i \in \mathcal{I}_{t}(W)}|W|^{-1 / q} \int_{W_{i}^{t}} f J_{W_{i}^{t}} \Phi_{t} d m_{W} .
\end{aligned}
$$

We estimate the terms in the sum over $k$ using the weak norm and the terms corresponding to $\mathcal{I}_{t}(W)$ using the strong stable norm,

$$
\begin{aligned}
\sum_{i}|W|^{-1 / q} \int_{W_{i}^{t}} f J_{W_{i}^{t}} \Phi_{t} d m_{W} \leq & C \sum_{k=0}^{\left\lfloor t / \tau_{\min }\right\rfloor} \sum_{j \in L_{k}(W, t)} \sum_{i \in A_{t}\left(W_{j}^{t} t_{k}\right)}|f|_{w} \frac{\left|J_{W_{i}^{t}} \Phi_{t}\right|_{L^{\infty}\left(W_{i}^{t}\right)}}{|W|^{1 / q}} \\
& +C \sum_{i \in \mathcal{I}_{t}(W)} \frac{\left|W_{i}^{t}\right|^{1 / q}}{|W|^{1 / q}}\|f\|_{s}\left|J_{W_{i}^{t}} \Phi_{t}\right|_{L^{\infty}\left(W_{i}^{t}\right)} .
\end{aligned}
$$

In the first sum above corresponding to $k \geq 0$, we have as in the proof of Lemma 3.8

$$
\left|J_{W_{i}^{t}} \Phi_{t}\right|_{L^{\infty}\left(W_{i}^{t}\right)} \leq\left|J_{W_{i}^{t}} \Phi_{k \tau_{\min }}\right|_{L^{\infty}\left(W_{i}^{t}\right)}\left|J_{W_{j}^{t} t_{k}} \Phi_{t_{k}}\right|_{L^{\infty}\left(W_{j}^{t}{ }^{t}\right)} .
$$

Thus using Lemma 3.8(a) for $1 / q_{0}=0$ from time $t_{k}$ to time $t$,

$$
\begin{aligned}
\sum_{k=0}^{\left\lfloor t / \tau_{\min }\right\rfloor} & \sum_{j \in L_{k}(W, t)} \sum_{i \in A_{t}\left(W_{j}^{t}\right)}|W|^{-1 / q}\left|J_{W_{i}^{t}} \Phi_{t}\right|_{L^{\infty}\left(W_{i}^{t}\right)} \\
& \leq \sum_{k=0}^{\left\lfloor t / \tau_{\min }\right\rfloor} \sum_{j \in L_{k}(W, t)}\left|J_{W_{j}^{t} k} \Phi_{t_{k}}\right|_{L^{\infty}\left(W_{j}^{t}\right)}|W|^{-1 / q} \sum_{i \in A_{t}\left(W_{j}^{t_{k}}\right)}\left|J_{W_{i}^{t}} \Phi_{k \tau_{\min }}\right|_{L^{\infty}\left(W_{i}^{t}\right)} \\
& \leq C L_{0}^{-1 / q} \sum_{k=0}^{\left\lfloor t / \tau_{\min }\right\rfloor} \sum_{j \in L_{k}(W, t)} \frac{\left|W_{j}^{t_{k}}\right|^{1 / q}}{|W|^{1 / q}}\left|J_{W_{j}^{t_{k}}} \Phi_{t_{k}}\right|_{L^{\infty}\left(W_{j}^{t}\right)^{t}} \lambda^{k \tau_{\min }},
\end{aligned}
$$


since $\left|W_{j}^{t_{k}}\right| \geq L_{0} / 3$. Since each $L_{k}(W, t) \subset \mathcal{G}_{t_{k}}(W)$ by definition, the sum over $j$ is bounded independently of $k$ and $t$ by Lemma 3.8(b). The sum over $k$ is also bounded independently of $t$. Finally, for the sum corresponding to $\mathcal{I}_{t}(W)$ in (4.12), we use Lemma 3.8(a) with $q_{0}=q$ to get

$$
\sum_{i \in \mathcal{I}_{t}(W)} \frac{\left|W_{i}^{t}\right|^{1 / q}}{|W|^{1 / q}}\left|J_{W_{i}^{t}} \Phi_{t}\right|_{L^{\infty}\left(W_{i}^{t}\right)} \leq C \lambda^{t(1-1 / q)} .
$$

Putting this estimate together with (4.13) in (4.12), we obtain

$$
\sum_{i}|W|^{-1 / q}\left|\int_{W_{i}^{t}} f J_{W_{i}^{t}} \Phi_{t} d m_{W}\right| \leq C L_{0}^{-1 / q}|f|_{w}+C\|f\|_{s} \lambda^{t(1-1 / q)} .
$$

Finally, combining (4.11) and (4.14) with (4.9) yields

$$
\int_{W} \mathcal{L}_{t} f \psi d m_{W} \leq C\left(\Lambda^{-\beta t}+\lambda^{t(1-1 / q)}\right)\|f\|_{s}+C L_{0}^{-1 / q}|f|_{w}
$$

Taking the supremum over $W \in \mathcal{W}^{s}$ and $\psi \in \mathcal{C}^{\beta}(W)$ with $|W|^{1 / q}|\psi|_{\mathcal{C}^{\beta}(W)} \leq 1$ proves (4.2).

We prove (4.3). Let $f$ and $t>0$ be as before. Fix $W \in \mathcal{W}^{s}$ and $\psi \in \mathcal{C}^{\alpha}(W)$ with $|\psi|_{\mathcal{C}^{\alpha}(W)} \leq 1$. Then for $f \in \mathcal{C}^{0}\left(\Omega_{0}\right) \cap \mathcal{B}^{0}$,

$$
\left.\int_{W} \partial_{s}\left(\left(\mathcal{L}_{t} f\right) \circ \Phi_{s}\right)\right|_{s=0} \psi d m_{W}=\int_{W}\left(\left.\partial_{s}\left(f \circ \Phi_{s}\right)\right|_{s=0} \circ \Phi_{-t}\right) \psi d m_{W}
$$

where we used the group property (2.11). Now,

$$
\begin{aligned}
\int_{W}\left(\left.\partial_{s}\left(f \circ \Phi_{s}\right)\right|_{s=0} \circ \Phi_{-t}\right) \psi d m_{W} & =\sum_{W_{i} \in \mathcal{G}_{t}(W)} \int_{W_{i}}\left(\left.\partial_{s}\left(f \circ \Phi_{s}\right)\right|_{s=0}\right) \cdot J_{W_{i}} \Phi_{t} \cdot \psi \circ \Phi_{t} d m_{W} \\
& \leq \sum_{i}\|f\|_{0}\left|J_{W_{i}} \Phi_{t}\right|_{\mathcal{C}^{\alpha}\left(W_{i}\right)}\left|\psi \circ \Phi_{t}\right|_{\mathcal{C}^{\alpha}\left(W_{i}\right)} \leq C\|f\|_{0},
\end{aligned}
$$

where we estimated the Hölder norms of the functions as for (4.1) and used Lemma 3.8(b) to bound the sum. Taking the appropriate suprema over $W$ and $\psi$ proves (4.3).

4.2. Unstable norm estimate for $\mathcal{L}_{t}$. Fix $f, t$, and $W$ as in the previous subsection, let $\varepsilon \leq 1$, and consider two curves $W^{1}, W^{2} \in \mathcal{W}^{s}$ with $d_{\mathcal{W}^{s}}\left(W^{1}, W^{2}\right) \leq \varepsilon$. For $t>0$, we will use the collision map to partition $\Phi_{-t}\left(W^{\ell}\right)$ into "matched" pieces $U_{j}^{\ell}$ and "unmatched" pieces $V_{i}^{\ell}, \ell=1,2$. Define $\widetilde{W}_{i}^{\ell}=P^{+}\left(W_{i}^{\ell}\right)$ for each $W_{i}^{\ell} \in \mathcal{G}_{t}\left(W^{\ell}\right)$ and $\widetilde{W}^{\ell}=P^{+}\left(W^{\ell}\right)$. Note that $\widetilde{W}_{i}^{\ell} \in \widetilde{\mathcal{G}}_{n}\left(\widetilde{W}^{\ell}\right)$ (defined in the beginning of the proof of Lemma 3.8) for some $t / \tau_{\max } \leq n \leq t / \tau_{\min }$. We recall the matching of stable curves for the map used in [DZ1, §4.3]. Note that $\tilde{d}_{\mathcal{W}^{s}}\left(\widetilde{W}^{1}, \widetilde{W}^{2}\right) \leq \varepsilon$ by definition of $d_{\mathcal{W}^{s}}$.

Recall from Definition 2.6, $\mathcal{S}_{0}=\{(r, \varphi) \in \mathcal{M}: \varphi= \pm \pi / 2\}$ and $\mathcal{S}_{-n}^{\mathbb{H}}=\cup_{i=0}^{n} T^{i}\left(\mathcal{S}_{0} \cup \cup_{k \geq k_{0}} \partial \mathbb{H}_{k}\right)$ denotes the extended singularity set for $T^{-n}$, where we include the boundaries of the homogeneity strips to ensure applicability of Lemma 3.5. Let $\omega$ be a connected component of $\widetilde{W}^{1} \backslash \mathcal{S}_{-n}^{\mathbb{H}}$. To each point $z=(r, \varphi) \in T^{-n} \omega$, we associate a vertical line segment $\gamma_{z}$ of length at most $C \Lambda_{0}^{-n} \varepsilon$ such that its image $T^{n} \gamma_{z}$, if not cut by a singularity or the boundary of a homogeneity strip, will have length $C \varepsilon$. By [CM, §4.4], all the tangent vectors to $T^{i} \gamma_{z}$ lie in the unstable cone $C^{u}\left(T^{i} z\right)$ for each $i \geq 1$ so that they remain uniformly transverse to the stable cone and enjoy minimum expansion given by the factor $C \Lambda_{0}^{i}$.

Doing this for each connected component of $\widetilde{W}^{1} \backslash \mathcal{S}_{-n}^{\mathbb{H}}$, we subdivide $\widetilde{W}^{1} \backslash \mathcal{S}_{-n}^{\mathbb{H}}$ into a countable collection of subintervals of points for which $T^{n} \gamma_{z}$ intersects $\widetilde{W}^{2} \backslash \mathcal{S}_{-n}^{\mathbb{H}}$ and subintervals for which this is not the case. This induces a matching partition on $\widetilde{W}^{2} \backslash \mathcal{S}_{-n}^{\mathbb{H}}$. 
We denote by $\widetilde{V}_{i}^{\ell}$ the pieces in $T^{-n} \widetilde{W}^{\ell}$ which are not matched up by this process and note that the images $T^{n} \widetilde{V}_{i}^{\ell}$ occur either at the endpoints of $\widetilde{W}^{\ell}$ or because the vertical segment $\gamma_{z}$ has been cut by a singularity. In both cases, the length of the curves $T^{n} \widetilde{V}_{i}^{\ell}$ can be at most $C \varepsilon$ due to the uniform transversality of $\mathcal{S}_{-n}^{\mathbb{H}}$ (see [CM, Prop. 4.41]) with the map-stable cone $C_{z}^{s}$ and of $C_{z}^{s}$ with $C_{z}^{u}$.

In the remaining pieces the foliation $\left\{T^{n} \gamma_{z}\right\}_{z \in T^{-n} \widetilde{W}^{1}}$ provides a one-to-one correspondence between points in $\widetilde{W}^{1}$ and $\widetilde{W}^{2}$. We further subdivide these pieces in such a way that the lengths of their images under $T^{-i}$ are less than $L_{0}$ for each $0 \leq i \leq n$ and the pieces are pairwise matched by the foliation $\left\{\gamma_{z}\right\}$. We call these matched pieces $\widetilde{U}_{j}^{\ell}$. Possibly changing the constant $L_{0} / 2$ to $L_{0} / C$ for some uniform constant $C>1$ (depending only on the distortion constant and the angle between stable and unstable cones) in the definition of $\widetilde{\mathcal{G}}_{n}\left(\widetilde{W}^{\ell}\right)$, we may arrange it so that $\widetilde{U}_{j}^{\ell} \subset \widetilde{W}_{i}^{\ell, n}$ for some $\widetilde{W}_{i}^{\ell, n} \in \widetilde{\mathcal{G}}_{n}\left(\widetilde{W}^{\ell}\right)$ and $\widetilde{V}_{k}^{\ell} \subset \widetilde{W}_{i^{\prime}}^{\ell, n}$ for some $\widetilde{W}_{i^{\prime}}^{\ell, n} \in \widetilde{\mathcal{G}}_{n}\left(\widetilde{W}^{\ell}\right)$ for all $j, k \geq 1$ and $\ell=1,2$. There is at most one $\widetilde{U}_{j}^{\ell}$ and two $\widetilde{V}_{j}^{\ell}$ per $\widetilde{W}_{i}^{\ell, n} \in \widetilde{\mathcal{G}}_{n}\left(\widetilde{W}^{\ell}\right)$.

In this way, we write $\widetilde{W}^{\ell}=\left(\cup_{j} T^{n} \widetilde{U}_{j}^{\ell}\right) \cup\left(\cup_{i} T^{n} \widetilde{V}_{i}^{\ell}\right)$. The images $T^{n} \widetilde{V}_{i}^{\ell}$ of the unmatched pieces must be short while the images of the matched pieces $\widetilde{U}_{j}^{\ell}$ may be long or short.

Returning to the components of $\Phi_{-t}\left(W^{\ell}\right)$ for the flow, $\mathcal{G}_{t}\left(W^{\ell}\right)$, note that if $W_{i}^{\ell}$ is not part of a curve in $\widehat{\mathcal{G}}_{t}\left(W^{\ell}\right)$ undergoing a collision at time $-t$, then $P^{+}\left(W_{i}^{\ell}\right)$ is a union of at-most one matched and two unmatched curves $\widetilde{U}_{j}^{\ell}$ and $\widetilde{V}_{k}^{\ell}$ as described above. We may thus define $U_{j}^{\ell}$ as the subset of $W_{i}^{\ell}$ such that $P^{+}\left(U_{j}^{\ell}\right)=\widetilde{U}_{j}^{\ell}$ and $V_{k}^{\ell}$ as a connected subset of $W_{i}^{\ell}$ such that $P^{+}\left(V_{k}^{\ell}\right)=\widetilde{V}_{k}^{\ell}$. If $W_{i}^{\ell}$ is part of a curve in $\widehat{\mathcal{G}}_{t}\left(W^{\ell}\right)$ undergoing a collision at time $-t$, then $P^{+}\left(W_{i}^{\ell}\right)$ may correspond to only part of a curve $\widetilde{U}_{j}^{\ell}$ and at most two $\widetilde{V}_{k}^{\ell}$ as described above. However, we will still consider those pieces as matched or unmatched as defined.

With the above matching, $d_{\mathcal{W}^{s}}\left(U_{j}^{1}, U_{j}^{2}\right)$ may be infinite since there may not be an unstable curve connecting the two. However, there is an unstable curve $\widetilde{W}_{j}^{u}$ for the map connecting $\widetilde{U}_{j}^{1}$ and $\widetilde{U}_{j}^{2}$. Note that $\widetilde{W}_{j}^{u}$ is the trace of an unstable curve for the flow $W_{j}^{u}$ which has nonempty intersection with $U_{j}^{1}$. Although $W_{j}^{u}$ may not intersect $U_{j}^{2}$, the weak unstable manifold containing $W_{j}^{u}$ does intersect $U_{j}^{2}$, since $P^{+}\left(W_{j}^{u}\right) \cap P^{+}\left(U_{j}^{2}\right)$ intersect. Thus there is a time $s_{j}$ so that

$$
\Phi_{s_{j}}\left(U_{j}^{2}\right) \cap W_{j}^{u} \neq \emptyset .
$$

For $\psi_{\ell}$ on $W^{\ell}$ with $\left|\psi_{\ell}\right|_{C^{\alpha}\left(W^{\ell}\right)} \leq 1$ and $d\left(\psi_{1}, \psi_{2}\right)=0$, with the above construction we must bound

$$
\begin{aligned}
\mid \int_{W^{1}} \mathcal{L}_{t} h \psi_{1} d m_{W} & -\int_{W^{2}} \mathcal{L}_{t} h \psi_{2} d m_{W}\left|\leq \sum_{\ell, k}\right| \int_{V_{k}^{\ell}} f J_{V_{k}^{\ell}} \Phi_{t} \psi_{\ell} \circ \Phi_{t} d m_{W} \mid \\
& +\sum_{j}\left|\int_{U_{j}^{1}} f J_{U_{j}^{1}} \Phi_{t} \psi_{1} \circ \Phi_{t} d m_{W}-\int_{U_{j}^{2}} f J_{U_{j}^{2}} \Phi_{t} \psi_{2} \circ \Phi_{t} d m_{W}\right| .
\end{aligned}
$$

We do the estimate over the unmatched pieces $V_{k}^{\ell}$ first using the strong stable norm. To do this, we group the $V_{k}^{\ell}$ according to when the associated vertical segments were most recently cut as follows (notice that we may just as well define the vertical segments $\gamma_{z}$ on $T^{-n} \widetilde{W}^{2}$ as on $\left.T^{-n} \widetilde{W}^{1}\right)$. For $z \in T^{-n} \widetilde{W}^{\ell}$, let $\gamma_{z}^{j}$ denote the component of $T^{j} \gamma_{z}$ still connected to $T^{j-n} \widetilde{W}^{\ell}$. Define

$$
A^{\ell}(i)=\left\{k:\left(\cup_{z \in \widetilde{V}_{k}^{\ell}} \gamma_{z}^{n-i-1}\right) \cap\left(\mathcal{S}_{1}^{\mathbb{H}} \backslash \mathcal{S}_{0}^{\mathbb{H}}\right) \neq \emptyset \text { and } i \in[0, n-1] \text { is minimal for this property }\right\} .
$$


Let $S$ be the singularity curve which intersects $\left(\cup_{z \in \widetilde{V}_{k}^{\ell}} \gamma_{z}^{n-i-1}\right)$ for some $k \in A^{\ell}(i)$. Then $T S$ is an element of $\mathcal{S}_{-1}^{\mathbb{H}}$ and since the curves $\gamma_{z}^{n-i}$ have length at most $C \Lambda_{0}^{-i} \varepsilon$, by the uniform transversality of stable curves with curves in $\mathcal{S}_{-1}^{\mathbb{H}}$, we have $\left|T^{n-i} \widetilde{V}_{k}^{\ell}\right| \leq C \varepsilon \Lambda_{0}^{-i}$.

Next we subdivide the interval $[0, t]$ into times $t_{j}=t-j \tau_{\min } / 2, j=0, \ldots, 2\left\lfloor\frac{t}{\tau_{\min }}\right\rfloor$. For each $k \in A^{\ell}(i)$, there exists at least one $j_{k}$ such that $\Phi_{j_{k} \tau_{\min } / 2}\left(V_{k}^{\ell}\right)$ is not undergoing a collision (due to the choice $L_{0} \leq \tau_{\min } / 4$ from Lemma 3.8), and $P^{+}\left(\Phi_{j_{k} \tau_{\min } / 2}\left(V_{k}^{\ell}\right)\right)=T^{n-i} \widetilde{V}_{k}^{\ell}$. It follows from Lemma 3.4 that $\left|\Phi_{j_{k} \tau_{\min } / 2}\left(V_{k}^{\ell}\right)\right| \leq C \varepsilon \Lambda_{0}^{-i}$.

In addition, the $i$ th collision must occur at a time $s \in\left[\left\lfloor i \tau_{\min }\right\rfloor,\left\lfloor i \tau_{\max }\right\rfloor+1\right]$ so that the number of $t_{j}$ corresponding to a fixed $i$ is bounded by a uniform constant times $i$. Now for $k \in A^{\ell}(i)$,

$$
\begin{aligned}
\int_{V_{k}^{\ell}} f J_{V_{k}^{\ell}} \Phi_{t} \psi_{\ell} \circ \Phi_{t} d m_{W} & =\int_{\Phi_{j_{k} \tau_{\min } / 2}\left(V_{k}^{\ell}\right)} \underset{j_{k} \tau_{\min } / 2}{ } f J_{\Phi_{j_{k} \tau_{\min } / 2}\left(V_{k}^{\ell}\right)} \Phi_{t_{j_{k}}} \psi_{\ell} \circ \Phi_{t_{j_{k}}} d m_{W} \\
& \leq C\left\|\mathcal{L}_{j_{k} \tau_{\min } / 2} f\right\|_{s}\left|\Phi_{j_{k} \tau_{\min } / 2}\left(V_{k}^{\ell}\right)\right|^{1 / q}\left|J_{\Phi_{j_{k} \tau_{\min } / 2}\left(V_{k}^{\ell}\right)} \Phi_{t_{j_{k}}}\right|_{\mathcal{C}^{\beta}} \\
& \leq C\|f\|_{s} \varepsilon^{1 / q} \Lambda_{0}^{-i / q}\left|J_{\Phi_{j_{k} \tau_{\min } / 2}\left(V_{k}^{\ell}\right)} \Phi_{t_{j_{k}}}\right|_{L^{\infty}\left(\Phi_{j_{k} \tau_{\min } / 2}\left(V_{k}^{\ell}\right)\right)},
\end{aligned}
$$

where we have used (4.2) to bound $\left\|\mathcal{L}_{j_{k} \tau_{\min } / 2} f\right\|_{s}$, Lemma 3.6 to estimate $\left|\psi_{\ell} \circ \Phi_{t_{j_{k}}}\right|_{\mathcal{C}^{\beta}} \leq$ $C_{1}\left|\psi_{\ell}\right|_{\mathcal{C}^{\alpha}\left(W^{\ell}\right)}$, and (4.7).

In order to sum over the relevant $\ell$ and $k$, we fix $i$, define $J(i)$ to be the set of $j$ possible for the $i$ th collision and $K(j)$ to be the set of $k$ for which $j_{k}=j$. Thus using (4.18), we estimate

$$
\begin{aligned}
& \sum_{\ell, k}\left|\int_{V_{k}^{\ell}} f J_{V_{k}^{\ell}} \Phi_{t} \psi_{\ell} \circ \Phi_{t} d m_{W}\right| \\
& \quad \leq C\|f\|_{s} \varepsilon^{1 / q} \sum_{\ell, i} \sum_{j \in J(i)} \sum_{k \in K(j)} \Lambda_{0}^{-i / q}\left|J_{\Phi_{j \tau_{\min } / 2}\left(V_{k}^{\ell}\right)} \Phi_{t_{j}}\right|_{L^{\infty}\left(\Phi_{j \tau_{m i n} / 2}\left(V_{k}^{\ell}\right)\right)} \\
& \quad \leq C\|f\|_{s} \varepsilon^{1 / q} \sum_{\ell, i} \sum_{j \in J(i)} \Lambda_{0}^{-i / q} \leq C\|f\|_{s} \varepsilon^{1 / q} \sum_{\ell, i} i \Lambda_{0}^{-i / q} \leq C \varepsilon^{1 / q}\|f\|_{s}
\end{aligned}
$$

where for the sum over $k$, we have noted that $\Phi_{j \tau_{\min } / 2}\left(V_{k}^{\ell}\right) \subset \Phi_{-t_{j}}\left(W^{\ell}\right)$, and we have applied Lemma 3.8(b) with $1 / q_{0}=0$, since there are at most two such curves corresponding to each element of $\mathcal{G}_{t_{j}}\left(W^{\ell}\right)$; for the sum over $j$, we have used the fact that $\# J(i) \leq C i$ for some $C>0$ depending on $\tau_{\max } / \tau_{\min }$.

Next, we must estimate the second term on the right-hand side of (4.17). Recall that $\widetilde{U}_{j}^{\ell}=$ $P^{+}\left(U_{j}^{\ell}\right)$ can be represented as the graph $G_{j}^{\ell}(r)=\left(r, \varphi_{\widetilde{U}_{j}^{\ell}}(r)\right)$ of a function over some $r$-interval $I_{j}$. By the definition of matched pieces, $\widetilde{U}_{j}^{1}$ and $\widetilde{U}_{j}^{2}$ are defined over the same interval $I_{j}$. Let $S_{j}^{1}(r)=\Phi_{-t(r)} \circ G_{j}^{1}(r)$ denote the (invertible) map from $I_{j}$ to $U_{j}^{1}$ and $S_{j}^{2}=\Phi_{-t(r)} \circ G_{j}^{\ell}(r)$ denote the map from $I_{j}$ to $\Phi_{s_{j}}\left(U_{j}^{2}\right)$. For each $j$, define

$$
\phi_{j}=\left[J_{U_{j}^{1}} \Phi_{t} \cdot \psi_{1} \circ \Phi_{t}\right] \circ S_{j}^{1} \circ\left(S_{j}^{2}\right)^{-1} .
$$


The function $\phi_{j}$ is well-defined on $U_{j}^{2}$, and recalling $s_{j}$ from (4.16), we estimate,

$$
\begin{aligned}
\mid \int_{U_{j}^{1}} f J_{U_{j}^{1}} \Phi_{t} \psi_{1} \circ \Phi_{t} & -\int_{U_{j}^{2}} f J_{U_{j}^{2}} \Phi_{t} \psi_{2} \circ \Phi_{t}|\leq| \int_{U_{j}^{1}} f J_{U_{j}^{1}} \Phi_{t} \psi_{1} \circ \Phi_{t}-\int_{\Phi_{s_{j}}\left(U_{j}^{2}\right)} f \phi_{j} \mid \\
& +\left|\int_{\Phi_{s_{j}}\left(U_{j}^{2}\right)} f\left(\phi_{j}-J_{\Phi_{s_{j}}\left(U_{j}^{2}\right)} \Phi_{t-s_{j}} \psi_{2} \circ \Phi_{t-s_{j}}\right)\right| \\
& +\left|\int_{\Phi_{s_{j}}\left(U_{j}^{2}\right)} f J_{\Phi_{s_{j}} U_{j}^{2}} \Phi_{t-s_{j}} \psi_{2} \circ \Phi_{t-s_{j}}-\int_{U_{j}^{2}} f J_{U_{j}^{2}} \Phi_{t} \psi_{2} \circ \Phi_{t}\right| .
\end{aligned}
$$

We estimate the first term in equation (4.20) using the unstable norm. Lemma 3.6 and the distortion bounds given by (4.7) imply that

$$
\left|J_{U_{j}^{1}} \Phi_{t} \cdot \psi_{1} \circ \Phi_{t}\right|_{\mathcal{C}^{\alpha}\left(U_{j}^{1}\right)} \leq C\left|J_{U_{j}^{1}} \Phi_{t}\right|_{L^{\infty}\left(U_{j}^{1}\right)} .
$$

Similarly, since $\left|S_{j}^{1} \circ\left(S_{j}^{2}\right)^{-1}\right|_{\mathcal{C}^{1}} \leq C$ by Lemma 3.4, we have

$$
\left|\phi_{j}\right|_{\mathcal{C}^{\alpha}\left(U_{j}^{2}\right)} \leq\left|J_{U_{j}^{1}} \Phi_{t} \cdot \psi_{1} \circ \Phi_{t}\right|_{\mathcal{C}^{\alpha}\left(U_{j}^{1}\right)}\left|S_{j}^{1} \circ\left(S_{j}^{2}\right)^{-1}\right|_{\mathcal{C}^{1}} \leq C\left|J_{U_{j}^{1}} T^{n}\right|_{L^{\infty}\left(U_{j}^{1}\right)}
$$

By the definition of $\phi_{j}$ and $d(\cdot, \cdot)$,

$$
d\left(J_{U_{j}^{1}} \Phi_{t} \psi_{1} \circ \Phi_{t}, \phi_{j}\right)=\left|\left[J_{U_{j}^{1}} \Phi_{t} \psi_{1} \circ \Phi_{t}\right] \circ S_{j}^{1}-\phi_{j} \circ S_{j}^{2}\right|_{\mathcal{C}^{0}\left(I_{j}\right)}=0 .
$$

To complete the estimate on the first term of (4.20), we need the following lemma:

Lemma 4.3 (Lemma 4.2 of [DZ1]). There exists $C>0$, independent of $n, W^{1}$, and $W^{2}$, such that for each $j$,

$$
\tilde{d}_{\mathcal{W}}\left(\widetilde{U}_{j}^{1}, \widetilde{U}_{j}^{2}\right) \leq C \Lambda_{0}^{-n} n \varepsilon=: \varepsilon_{1} .
$$

Since $P^{+}\left(\Phi_{s_{j}}\left(U_{j}^{2}\right)\right)=\widetilde{U}_{j}^{2}$, it follows from the definition of $d_{\mathcal{W}^{s}}$ that $d_{\mathcal{W}^{s}}\left(U_{j}^{1}, \Phi_{s_{j}}\left(U_{j}^{2}\right)\right) \leq \varepsilon_{1}$ as well. In view of (4.21), we renormalize the test functions by $R_{j}=C\left|J_{U_{j}^{1}} \Phi_{t}\right|_{\mathcal{C}^{0}\left(U_{j}^{1}\right)}$. Then we apply the definition of the unstable norm with $\varepsilon_{1}$ in place of $\varepsilon$. Thus,

$$
\begin{aligned}
& \sum_{j}\left|\int_{U_{j}^{1}} f J_{U_{j}^{1}} \Phi_{t} \psi_{1} \circ \Phi_{t}-\int_{\Phi_{s_{j}}\left(U_{j}^{2}\right)} f \phi_{j}\right| \\
& \leq C \varepsilon_{1}^{1 / q}\|f\|_{u} \sum_{j}\left|J_{U_{j}^{1}} \Phi_{t}\right|_{L^{\infty}\left(U_{j}^{1}\right)} \leq C\|f\|_{u} t^{\gamma} \Lambda^{-t \gamma} \varepsilon^{\gamma},
\end{aligned}
$$

using the fact that $\left\lfloor\frac{t}{\tau_{\max }}\right\rfloor \leq n \leq\left\lfloor\frac{t}{\tau_{\min }}\right\rfloor+1$ and the sum is bounded by Lemma 3.8 (b) for $1 / q_{0}=0$ since there is at most one matched piece $U_{j}^{1}$ corresponding to each curve $W_{i}^{1} \in \mathcal{G}_{t}\left(W^{1}\right)$.

Next we estimate the second term in (4.20) using the strong stable norm.

$$
\begin{aligned}
& \left|\int_{\Phi_{s_{j}}\left(U_{j}^{2}\right)} f\left(\phi_{j}-J_{\Phi_{s_{j}}\left(U_{j}^{2}\right)} \Phi_{t-s_{j}} \psi_{2} \circ \Phi_{t-s_{j}}\right)\right| \\
& \leq\|f\|_{s}\left|\Phi_{s_{j}}\left(U_{j}^{2}\right)\right|^{1 / q}\left|\phi_{j}-J_{\Phi_{s_{j}}\left(U_{j}^{2}\right)} \Phi_{t-s_{j}} \cdot \psi_{2} \circ \Phi_{t-s_{j}}\right|_{\mathcal{C}^{\beta}\left(\Phi_{s_{j}}\left(U_{j}^{2}\right)\right)} .
\end{aligned}
$$


Using the fact that $\left|S_{j}^{1}\right|_{\mathcal{C}^{1}}\left|\left(S_{j}^{2}\right)^{-1}\right|_{\mathcal{C}^{1}} \leq C$ and $\left|\psi_{1} \circ \Phi_{t}\right|_{\mathcal{C}^{\beta}\left(U_{j}^{1}\right)} \leq C|\psi|_{\mathcal{C}^{\beta}\left(W^{1}\right)} \leq C$, we split the estimate on the norm of the test function,

$$
\begin{aligned}
\mid \phi_{j}-J_{\Phi_{s_{j}}\left(U_{j}^{2}\right)} \Phi_{t-s_{j}} & \left.\cdot \psi_{2} \circ \Phi_{t-s_{j}}\right|_{\mathcal{C}^{\beta}\left(\Phi_{s_{j}}\left(U_{j}^{2}\right)\right)} \leq C\left|J_{U_{j}^{1}} \Phi_{t} \circ S_{j}^{1}-J_{\Phi_{s_{j}}\left(U_{j}^{2}\right)} \Phi_{t-s_{j}} \circ S_{j}^{2}\right|_{\mathcal{C}^{\beta}\left(I_{j}\right)} \\
& +C\left|J_{\Phi_{s_{j}}\left(U_{j}^{2}\right)} \Phi_{t-s_{j}}\right|_{\mathcal{C}^{\beta}\left(\Phi_{s_{j}}\left(U_{j}^{2}\right)\right)}\left|\psi_{1} \circ \Phi_{t} \circ S_{j}^{1}-\psi_{2} \circ \Phi_{t-s_{j}} \circ S_{j}^{2}\right|_{\mathcal{C}^{\beta}\left(I_{j}\right)} .
\end{aligned}
$$

We will use the following lemma (which is the analogue of [DZ1, Lemmas 4.3 and 4.4]) to estimate the two differences above.

Lemma 4.4. There exists $C>0$ such that for each $j \geq 1$,

a) $\left|J_{U_{j}^{1}} \Phi_{t} \circ S_{j}^{1}-J_{\Phi_{s_{j}}\left(U_{j}^{2}\right)} \Phi_{t-s_{j}} \circ S_{j}^{2}\right|_{\mathcal{C}^{\beta}\left(I_{j}\right)} \leq C\left|J_{U_{j}^{2}} \Phi_{t}\right|_{C^{0}\left(U_{j}^{2}\right)} \varepsilon^{1 / 3-\beta} ;$

b) $\left|\psi_{1} \circ \Phi_{t} \circ S_{j}^{1}-\psi_{2} \circ \Phi_{t-s_{j}} \circ S_{j}^{2}\right|_{\mathcal{C}^{\beta}\left(I_{j}\right)} \leq C \varepsilon^{\alpha-\beta}$.

Proof. (a) Let $G_{\widetilde{W}^{\ell}}$ denote the graph representing $\widetilde{W}^{\ell}=P^{+}\left(W^{\ell}\right)$ and $S^{\ell}$ denote the map from $I_{\widetilde{W}^{\ell}}$ to $W^{\ell}, \ell=1,2$. Then $\Phi_{t} \circ S_{j}^{1}=S^{1} \circ G_{\widetilde{W}^{1}}^{-1} \circ T^{n} \circ G_{\widetilde{U}_{j}^{1}}$, so that

$$
J \Phi_{t} \circ S_{j}^{1}=J\left(S^{1} \circ G_{\widetilde{W}^{1}}^{-1}\right) \circ\left(T^{n} \circ G_{\widetilde{U}_{j}^{1}}\right) \cdot J_{\widetilde{U}_{j}^{1}} T^{n} \circ G_{\widetilde{U}_{j}^{1}} \cdot J G_{\widetilde{U}_{j}^{1}} \cdot\left(J S_{j}^{1}\right)^{-1},
$$

and a similar expression holds for $J_{\Phi_{s_{j}}\left(U_{j}^{2}\right)} \Phi_{t-s_{j}}$. All Jacobians except for $J_{\widetilde{U}_{j}^{1}} T^{n}$ are uniformly bounded by Lemma 3.4 (note that $S^{1} \circ G_{\widetilde{W}^{1}}^{-1}$ is the natural flow map from $P^{+}\left(W^{1}\right)$ to $W^{1}$ ).

Fixing $r \in I_{j}$, by [DZ1, eq. (4.16)], we have

$$
\left|J_{\widetilde{U}_{j}^{1}} T^{n} \circ G_{\widetilde{U}_{j}^{1}}(r)-J_{\widetilde{U}_{j}^{2}} T^{n} \circ G_{\widetilde{U}_{j}^{2}}(r)\right| \leq C\left|J_{\widetilde{U}_{j}^{2}} T^{n}\right|_{L^{\infty}\left(\widetilde{U}_{j}^{2}\right)} \varepsilon^{1 / 3} .
$$

Also, $\left|J G_{\widetilde{U}_{j}^{1}}(r)-J G_{\widetilde{U}_{j}^{2}}(r)\right| \leq C \varepsilon$ using (3.5) and the fact that $\tilde{d}_{\mathcal{W}^{s}}\left(\widetilde{U}_{j}^{1}, \widetilde{U}_{j}^{2}\right) \leq \varepsilon$. Now,

$$
\left|\left(J S_{j}^{1}\right)^{-1}(r)-\left(J S_{j}^{2}\right)^{-1}(r)\right|=\frac{1}{J S_{j}^{1}(r) J S_{j}^{2}(r)}\left|J S_{j}^{1}(r)-J S_{j}^{2}(r)\right|,
$$

and since by Lemma [3.4. $J S_{j}^{\ell}$ is bounded away from 0 , we can focus on the difference. Now in the notation of Lemma 3.4, $J S_{j}^{\ell}(r)=J_{\widetilde{U}_{j}^{\ell}} \Phi_{-t(r)}\left(G_{\widetilde{U}_{j}^{\ell}}(r)\right) J G_{\widetilde{U}_{j}^{\ell}}(r)$, and as already noted, the difference of $J G_{\widetilde{U}_{j}^{\ell}}$ is bounded by $\varepsilon$. Thus we focus on the difference involving $J_{\widetilde{U}_{j}^{\ell}} \Phi_{-t(r)}\left(G_{\widetilde{U}_{j}^{\ell}}(r)\right)$. Letting $z_{\ell}=G_{\widetilde{U}_{j}^{\ell}}(r)=\left(r_{\ell}, \varphi_{\ell}\right)$ and $\left(d r_{\ell}, d \varphi_{\ell}\right)$ denote the tangent vector to $\widetilde{U}_{j}^{\ell}$ at $z_{\ell}$, we have by (3.6)

$$
\ln \frac{J_{\widetilde{U}_{j}^{1}} \Phi_{-\tau\left(Z_{1}\right)}\left(z_{1}\right)}{J_{\widetilde{U}_{j}^{2}} \Phi_{-\tau\left(Z_{2}\right)}\left(z_{2}\right)}=\frac{1}{2} \ln \frac{\left(d r_{1}\right)^{2}\left[\left(\cos \varphi_{1}+\tau\left(Z_{1}\right) \mathcal{K}\left(r_{1}\right)-\tau\left(Z_{1}\right) \frac{d \varphi_{1}}{d r_{1}}\right)^{2}+\left(\mathcal{K}\left(r_{1}\right)-\frac{d \varphi_{1}}{d r_{1}}\right)^{2}\right]}{\left(d r_{2}\right)^{2}\left[\left(\cos \varphi_{2}+\tau\left(Z_{2}\right) \mathcal{K}\left(r_{2}\right)-\tau\left(Z_{2}\right) \frac{d \varphi_{2}}{d r_{2}}\right)^{2}+\left(\mathcal{K}\left(r_{2}\right)-\frac{d \varphi_{2}}{d r_{2}}\right)^{2}\right]},
$$

where $z_{\ell}=P^{+}\left(Z_{\ell}\right), Z_{1} \in U_{j}^{1}, Z_{2} \in \Phi_{s_{j}}\left(Z_{2}\right)$. Once again, we pair corresponding terms and estimate the differences. We have $d\left(z_{1}, z_{2}\right) \leq \varepsilon$ and $\left|\frac{d \varphi_{1}}{d r_{1}}-\frac{d \varphi_{2}}{d r_{2}}\right| \leq C \varepsilon$ since $\tilde{d}_{\mathcal{W}^{s}}\left(\widetilde{U}_{j}^{1}, \widetilde{U}_{j}^{2}\right) \leq \varepsilon$. This leaves only $\left|\tau\left(Z_{1}\right)-\tau\left(Z_{2}\right)\right|$ to estimate. Since by definition of $d_{\mathcal{W}^{s}}$, there is an unstable curve connecting $U_{j}^{1}$ and $\Phi_{s_{j}}\left(U_{j}^{2}\right)$, we have $d\left(Z_{1}, Z_{2}\right) \leq C \varepsilon$ and so $\left|\tau\left(Z_{1}\right)-\tau\left(Z_{2}\right)\right| \leq C \varepsilon^{1 / 2}$.

Finally, note that $J\left(S^{1} \circ G_{\widetilde{W}^{1}}^{-1}\right)=J_{\widetilde{W}^{1}} \Phi_{-\tau}$ and so satisfies the same estimate as above. Since $d\left(T^{n} \circ G_{\widetilde{U}_{j}^{1}}(r), T^{n} \circ G_{\widetilde{U}_{j}^{2}}(r)\right) \leq C \varepsilon$ by the uniform transversality of the foliation $\left\{T^{n} \gamma_{z}\right\}$, the triangle inequality together with Lemma 3.4 yields that this difference is also bounded by $C \varepsilon^{1 / 2}$.

These estimates together with Lemma 3.3 yield by (4.25),

$$
\left|J_{U_{j}^{1}} \Phi_{t} \circ S_{j}^{1}(r)-J_{\Phi_{s_{j}}\left(U_{j}^{2}\right)} \Phi_{t-s_{j}} \circ S_{j}^{2}(r)\right| \leq C\left|J_{U_{j}^{2}} \Phi_{t}\right|_{L^{\infty}\left(U_{j}^{2}\right)} \varepsilon^{1 / 3} .
$$


Now since both Jacobians satisfy bounded distortion along stable curves with exponent $1 / 3$ by Lemma 3.5. we use the Hölder interpolation from [DZ2, Lemma 4.3] to conclude the proof of part (a) the lemma.

(b) Fix $r \in I_{j}$ and set $z_{\ell}=v_{j}^{\ell}(r), Z_{\ell}=S_{j}^{\ell}(r), \ell=1,2$. Also, let $Y_{1}=\Phi_{t}\left(Z_{1}\right)$ and $Y_{2}=\Phi_{t-s_{j}}\left(Z_{2}\right)$ denote the images of $Z_{1}$ and $Z_{2}$ in $W^{1}$ and $W^{2}$, respectively. Now,

$$
\left|\psi_{1} \circ \Phi_{t} \circ S_{j}^{1}(r)-\psi_{2} \circ \Phi_{t-s_{j}} \circ S_{j}^{2}(r)\right|=\left|\psi_{1}\left(Y_{1}\right)-\psi_{2}\left(Y_{2}\right)\right| \text {. }
$$

Let $r^{\prime}$ denote the arclength coordinate of $w_{2}=P^{+}\left(Y_{2}\right)$ and set $w_{1}=P^{+}\left(Y_{1}\right)$. Note that $w_{1}$ does not have the same arclength coordinate as $w_{2}$ since the billiard map does not preserve vertical lines. Using the same notation as in part (a), let $w_{1}^{\prime}=G_{\widetilde{W}^{1}}\left(r^{\prime}\right)$ and $Y_{1}^{\prime}=S^{1}\left(r^{\prime}\right)$ denote the lifts of $r^{\prime}$ to $P^{+}\left(W^{1}\right)$ and $W^{1}$, respectively. Since $w_{1}^{\prime}$ and $w_{2}$ lie on the same vertical segment and $\tilde{d}_{\mathcal{W}^{s}}\left(P^{+}\left(W^{1}\right), P^{+}\left(W^{2}\right)\right) \leq \varepsilon$, we have $\left|w_{1}^{\prime}-w_{2}\right| \leq \varepsilon$. Also, since $z_{1}$ and $z_{2}$ line on the same vertical line segment, the segment connecting $w_{1}$ and $w_{2}$ lies in the map unstable cone and so is uniformly transverse to the stable cone. Thus $\left|w_{1}-w_{2}\right| \leq C \varepsilon$ and so by the triangle inequality, $\left|w_{1}-w_{1}^{\prime}\right| \leq C \varepsilon$. It then follows from Lemma 3.4 that $d\left(Y_{1}, Y_{1}^{\prime}\right) \leq C \varepsilon$. Putting these estimates together, and using the fact that $\left|\psi_{1} \circ S^{1}-\psi_{2} \circ S^{2}\right|_{\infty}=0$, we estimate,

$$
\begin{aligned}
\left|\psi_{1} \circ \Phi_{t} \circ S_{j}^{1}(r)-\psi_{2} \circ \Phi_{t-s_{j}} \circ S_{j}^{2}(r)\right| & \leq\left|\psi_{1}\left(Y_{1}\right)-\psi_{1}\left(Y_{1}^{\prime}\right)\right|+\left|\psi_{1}\left(Y_{1}^{\prime}\right)-\psi_{2}\left(Y_{2}\right)\right| \\
& \leq C d\left(Y_{1}, Y_{1}^{\prime}\right)^{\alpha} \leq C \varepsilon^{\alpha} .
\end{aligned}
$$

Finally, since $\psi_{1}$ and $\psi_{2}$ are Hölder continuous with exponent $\alpha$, we again use the Hölder interpolation from [DZ2, Lemma 4.3], to conclude part (b) of the lemma.

With Lemma 4.4 proved, (4.23), and (4.24) complete the estimate on the second term in (4.20). Indeed, we have

$$
\begin{aligned}
\sum_{j}\left|\int_{\Phi_{s_{j}}\left(U_{j}^{2}\right)} f\left(\phi_{j}-J_{\Phi_{s_{j}}\left(U_{j}^{2}\right)} \Phi_{t-s_{j}}\right) \psi_{2} \circ \Phi_{t-s_{j}}\right| & \leq \sum_{j} C\|f\|_{s}\left|\Phi_{s_{j}}\left(U_{j}^{2}\right)\right|^{1 / q}\left|J_{U_{j}^{2}} \Phi_{t}\right|_{L^{\infty}\left(U_{j}^{2}\right)} \varepsilon^{\alpha-\beta} \\
& \leq C\|f\|_{s} \varepsilon^{\alpha-\beta},
\end{aligned}
$$

where again the sum is bounded by Lemma 3.8 (b) (using that $\left|\Phi_{s_{j}}\left(U_{j}^{2}\right)\right|$ is comparable to $\left|U_{j}^{2}\right|$ ).

It remains to estimate the third term in (4.20), which we do using the neutral norm. For this, we first state and prove a needed bound on the times $s_{j}$ from (4.16),

Lemma 4.5. There exists $C>0$ such that $s_{j} \leq C \varepsilon^{1 / 2}$ for each $j$.

Proof. We first show that the collision times of $U_{j}^{1}$ and $U_{j}^{2}$ remain close throughout their orbits until time $t$. Let $n$ denote the number of collisions from time 0 to time $t$.

Let $Z \in \Phi_{t}\left(U_{j}^{1}\right), Y \in \Phi_{t}\left(U_{j}^{2}\right)$ be two points such that $P^{+}(Z)$ and $P^{+}(Y)$ are connected by one of the curves $T^{n}\left(\gamma_{z}\right)$ defined during the matching process at the beginning of this section. Since $d_{\mathcal{W}^{s}}\left(W^{1}, W^{2}\right) \leq \varepsilon$, we have $d\left(P^{+}(Z), P^{+}(Y)\right) \leq C \varepsilon$ and $d(Z, Y) \leq C \varepsilon$, for some uniform constant $C>0$. Now

$$
\begin{aligned}
\left|\tau^{-n}\left(P^{+}(Z)\right)-\tau^{-n}\left(P^{+}(Y)\right)\right| & \leq \sum_{i=0}^{n-1}\left|\tau^{-}\left(T^{-i} P^{+}(Z)\right)-\tau^{-}\left(T^{-i} P^{+}(Y)\right)\right| \\
& \leq \sum_{i=0}^{n-1} C \Lambda_{0}^{-i / 2} d\left(P^{+}(Z), P^{+}(Y)\right)^{1 / 2} \leq C^{\prime} \varepsilon^{1 / 2},
\end{aligned}
$$

due to the $1 / 2$-Hölder continuity of $\tau^{-}$(analogous to (3.7)) and the uniform expansion along unstable curves. Since $|\tau(Z)-\tau(Y)| \leq C d(Z, Y)^{1 / 2} \leq C \varepsilon^{1 / 2}$ and since $t=\tau\left(\Phi_{-t}(Z)\right)+$ 
$\tau^{-n}\left(P^{+}(Z)\right)-\tau(Z)$ with an analogous expression for $t$ in terms of $Y$, we conclude that $\mid \tau\left(\Phi_{-t} Z\right)-$ $\tau\left(\Phi_{-t} Y\right) \mid \leq C \varepsilon^{1 / 2}$. Thus it must be that $d\left(\Phi_{-t} Z, \Phi_{-t} Y\right) \leq C \varepsilon^{1 / 2}$.

Since $\Phi_{s_{j}}\left(U_{j}^{2}\right)$ and $U_{j}^{1}$ are connected by an unstable curve of length $C \varepsilon$, if we choose $Y$ in the above analysis to be the point in $U_{j}^{2}$ whose translate by $s_{j}$ belongs to this unstable curve, then the triangle inequality yields $s_{j} \leq C \varepsilon^{1 / 2}$, proving Lemma 4.5,

We may now estimate the second term in (4.20):

$$
\begin{aligned}
\int_{\Phi_{s_{j}}\left(U_{j}^{2}\right)} f J_{\Phi_{s_{j}}\left(U_{j}^{2}\right)} \Phi_{t-s_{j}} \psi_{2} \circ \Phi_{t-s_{j}} & -\int_{U_{j}^{2}} f J_{U_{j}^{2}} \Phi_{t} \psi_{2} \circ \Phi_{t} \\
& =\int_{0}^{s_{j}} \partial_{s} \int_{\Phi_{s}\left(U_{j}^{2}\right)} f J_{\Phi_{s}\left(U_{j}^{2}\right)} \Phi_{t-s} \psi_{2} \circ \Phi_{t-s} d s \\
& =\int_{0}^{s_{j}} \partial_{s} \int_{U_{j}^{2}} f \circ \Phi_{s} J_{U_{j}^{2}} \Phi_{t} \psi_{2} \circ \Phi_{t} d s .
\end{aligned}
$$

Thus, recalling (2.11), (with $s_{j} \leq C \sqrt{\epsilon}$ by Lemma 4.5.)

$$
\begin{gathered}
\int_{0}^{s_{j}} \int_{U_{j}^{2}} \partial_{s}\left(f \circ \Phi_{s}\right) J_{U_{j}^{2}} \Phi_{t} \psi \circ \Phi_{t} d m_{W} d s=\left.\int_{0}^{s_{j}} \int_{U_{j}^{2}}\left(\partial_{r} f \circ \Phi_{r}\right)\right|_{r=s} \circ \Phi_{s} J_{U_{j}^{2}} \Phi_{t}\left(\psi \circ \Phi_{t}\right) d m_{W} d s \\
\leq s_{j}\|f\|_{0}\left|J_{\left(U_{j}^{2}\right)} \Phi_{t}\left(\psi \circ \Phi_{t}\right)\right|_{\mathcal{C}^{\alpha}\left(U_{j}^{2}\right)} \leq C s_{j}\|f\|_{0}\left|J_{\left(U_{j}^{2}\right)} \Phi_{t}\right|_{L^{\infty}\left(U_{j}^{2}\right)},
\end{gathered}
$$

where once again, we have used Lemma 3.6 and (4.7).

We use Lemma 4.5 together with (4.27) and (4.28) to estimate the third term in (4.20),

$$
\begin{aligned}
\sum_{j}\left|\int_{\Phi_{s_{j}}\left(U_{j}^{2}\right)} f J_{\Phi_{s_{j}}\left(U_{j}^{2}\right)} \Phi_{t-s_{j}} \psi_{2} \circ \Phi_{t-s_{j}}-\int_{U_{j}^{2}} f J_{U_{j}^{2}} \Phi_{t} \psi_{2} \circ \Phi_{t}\right| & \leq \sum_{j} C\|f\|_{0} s_{j}\left|J_{U_{j}^{2}} \Phi_{t}\right|_{L^{\infty}\left(U_{j}^{2}\right)} \\
& \leq C \varepsilon^{1 / 2}\|f\|_{0},
\end{aligned}
$$

where again we have used Lemma $3.8(b)$.

Now we use this bound, together with (4.19), (4.22) and (4.26) to estimate (4.17)

$$
\begin{aligned}
\left|\int_{W^{1}} \mathcal{L}_{t} f \psi_{1} d m_{W}-\int_{W^{2}} \mathcal{L}_{t} f \psi_{2} d m_{W}\right| \leq C & \|f\|_{s} \varepsilon^{1 / q}+C\|f\|_{u} t^{\gamma} \Lambda^{-t \gamma} \varepsilon^{\gamma} \\
& +C\|f\|_{s} \varepsilon^{\alpha-\beta}+C\|f\|_{0} \varepsilon^{1 / 2} .
\end{aligned}
$$

Since $\gamma \leq \min \{1 / q, \alpha-\beta\}<1 / 3$, we divide through by $\varepsilon^{\gamma}$ and take the appropriate suprema to complete the proof of (4.4).

4.3. Strong continuity of $\mathcal{L}_{t}$ on $\mathcal{B}$. To complete this section, we state and prove the announced lemma on the regularity of $t \mapsto \mathcal{L}_{t}$. (See also the variant given in Lemma 4.7 below.)

Lemma 4.6 (Strong continuity). For each $f \in \mathcal{B}$ we have $\lim _{t \downarrow 0}\left\|\mathcal{L}_{t} f-f\right\|_{\mathcal{B}}=0$.

Proof. First we prove the statement for $f \in \mathcal{C}_{\sim}^{0} \cap \mathcal{C}^{2}\left(\Omega_{0}\right)$, then we extend it to general $f \in \mathcal{B}$. We fix $f \in \mathcal{C}_{\sim}^{0} \cap \mathcal{C}^{2}\left(\Omega_{0}\right)$ and consider each component of the norm separately.

To estimate the strong stable norm, fix $W \in \mathcal{W}^{s}$ and $\psi \in \mathcal{C}^{\beta}(W)$ with $|W|^{1 / q}|\psi|_{\mathcal{C}^{\beta}(W)} \leq 1$. Then 26

$$
\int_{W}\left(\mathcal{L}_{t} f-f\right) \psi d m_{W}=\int_{W} \int_{0}^{t} \partial_{s}\left(\mathcal{L}_{s} f\right) d s \psi d m_{W}
$$

\footnotetext{
26 Note for any curve $W$ and any $x$ in $\mathrm{W}$, the point $\Phi_{s}(x)$ only hits $\partial \Omega_{0}$ at finitely many times $s$, recall also that $f \in \mathcal{C}_{\sim}^{0}$.
} 
As usual, by the group property (see (2.11)), we have

$$
\partial_{s}\left(\mathcal{L}_{s} f\right)=\left.\partial_{r}\left(f \circ \Phi_{r}\right)\right|_{r=0} \circ \Phi_{-s}=(\nabla f \cdot \hat{\eta}) \circ \Phi_{-s}=\mathcal{L}_{s}(\nabla f \cdot \hat{\eta}),
$$

where $\hat{\eta}$ denotes the unit vector in the direction of the flow. Since $\nabla f \cdot \hat{\eta} \in \mathcal{C}^{1}\left(\Omega_{0}\right) \subset \mathcal{B}^{0}$ (it is not an element of $\mathcal{C}_{\sim}^{0}$ or $\mathcal{B}_{\sim}^{0}$ in general, but this is immaterial) we can apply (4.15) to estimate,

$$
\int_{W}\left(\mathcal{L}_{t} f-f\right) \psi d m_{W}=\int_{0}^{t} \int_{W} \mathcal{L}_{s}(\nabla f \cdot \hat{\eta}) \psi d m_{W} d s \leq C t\left(\|\nabla f \cdot \hat{\eta}\|_{s}+|\nabla f \cdot \hat{\eta}|_{w}\right),
$$

where (3.12) gives $\|\nabla f \cdot \hat{\eta}\|_{s}+|\nabla f \cdot \hat{\eta}|_{w} \leq|\nabla f \cdot \hat{\eta}|_{\mathcal{C}^{0}\left(\Omega_{0}\right)}$. Taking the supremum over $W$ and $\psi$ yields the estimate on the strong stable norm,

$$
\left\|\mathcal{L}_{t} f-f\right\|_{s} \leq C t|\nabla f \cdot \hat{\eta}|_{\mathcal{C}^{0}\left(\Omega_{0}\right)} .
$$

To estimate the unstable norm, fix $\varepsilon>0$ and let $W_{1}, W_{2} \in \mathcal{W}^{s}$ satisfy $d_{\mathcal{W}^{s}}\left(W_{1}, W_{2}\right)<\varepsilon$. Take $\psi_{i} \in \mathcal{C}^{\alpha}\left(W_{i}\right)$ with $\left|\psi_{i}\right|_{\mathcal{C}^{\alpha}(W)} \leq 1$ and $d\left(\psi_{1}, \psi_{2}\right)=0$. Then using again (4.30) and (4.29), we have

$$
\begin{aligned}
\int_{W_{1}}\left(\mathcal{L}_{t} f-f\right) \psi_{1} d m_{W} & -\int_{W_{2}}\left(\mathcal{L}_{t} f-f\right) \psi_{2} d m_{W} \\
& =\int_{W_{1}} \int_{0}^{t} \partial_{s}\left(\mathcal{L}_{s} f\right) d s \psi_{1} d m_{W}-\int_{W_{2}} \int_{0}^{t} \partial_{s}\left(\mathcal{L}_{s} f\right) d s \psi_{2} d m_{W} \\
& =\int_{0}^{t}\left(\int_{W_{1}} \mathcal{L}_{s}(\nabla f \cdot \hat{\eta}) \psi_{1} d m_{W}-\int_{W_{2}} \mathcal{L}_{s}(\nabla f \cdot \hat{\eta}) \psi_{2} d m_{W}\right) d s \\
& \leq t C\left(\|\nabla f \cdot \hat{\eta}\|_{s} \varepsilon^{1 / q}+\|\nabla f \cdot \hat{\eta}\|_{u} \varepsilon^{\gamma}+\|\nabla f \cdot \hat{\eta}\|_{s} \varepsilon^{\alpha-\beta}+\|\nabla f \cdot \hat{\eta}\|_{0} \varepsilon^{1 / 2}\right) .
\end{aligned}
$$

Dividing through by $\varepsilon^{\gamma}$ (recall that $\gamma<1 / 3$ ), recalling (3.12), and taking the appropriate suprema yields the bound $\left\|\mathcal{L}_{t} f-f\right\|_{u} \leq C t|\nabla f \cdot \hat{\eta}|_{\mathcal{C}^{1}\left(\Omega_{0}\right)}$.

Finally, to estimate the neutral norm, we fix $0<\left|r_{0}\right| \leq 1, W \in \mathcal{W}^{s}$ and $\psi \in \mathcal{C}^{\alpha}(W)$ with $|\psi|_{\mathcal{C}^{\alpha}(W)} \leq 1$. Then using (4.30) and the neutral estimate (4.3), we have,

$$
\begin{gathered}
\left.\int_{W} \partial_{r}\left(\left(\mathcal{L}_{t} f-f\right) \circ \Phi_{r}\right)\right|_{r=0} \psi d m_{W}=\left.\int_{W} \partial_{r}\left(\left(\int_{0}^{t} \mathcal{L}_{s}(\nabla f \cdot \hat{\eta}) d s\right) \circ \Phi_{r}\right)\right|_{r=0} \psi d m_{W} \\
=\left.\int_{0}^{t} \int_{W} \partial_{r}\left(\mathcal{L}_{s}(\nabla f \cdot \hat{\eta}) \circ \Phi_{r}\right)\right|_{r=0} \psi d m_{W} d s \leq C t\|\nabla f \cdot \hat{\eta}\|_{0} .
\end{gathered}
$$

Taken together, the estimates on the three components of the norm imply the bound $\| \mathcal{L}_{t} f-$ $f \|_{\mathcal{B}} \leq C t|\nabla f \cdot \hat{\eta}|_{\mathcal{C}^{1}\left(\Omega_{0}\right)}$, which proves the lemma for $f \in \mathcal{C}^{2}\left(\Omega_{0}\right) \cap \mathcal{C}_{\sim}^{0}$.

For more general $f \in \mathcal{B}$, we proceed by approximation. By the definition of $\mathcal{B}$, in particular (2.10), we can approach any $f$ in $\mathcal{B}$ by a sequence, $\mathcal{L}_{t_{n}}\left(h_{n}\right)$ with $h_{n} \in \mathcal{C}^{2}\left(\Omega_{0}\right) \cap \mathcal{C}_{\sim}^{0}$ and $t_{n} \geq 0$. Then we write

$$
\mathcal{L}_{t}(f)-f=\mathcal{L}_{t}\left(f-\mathcal{L}_{t_{n}} h_{n}\right)+\mathcal{L}_{t_{n}}\left(\mathcal{L}_{t}\left(h_{n}\right)-h_{n}\right)+\left(\mathcal{L}_{t_{n}} h_{n}-f\right) .
$$

By Proposition 4.1, there exists $\hat{C}$ so that $\left\|\mathcal{L}_{t} f\right\|_{\mathcal{B}} \leq \hat{C}\|f\|_{\mathcal{B}}$ for all $f \in \mathcal{B} \subset \mathcal{B}^{0}$. Thus the first and last term on the right-hand side above tend to zero in $\mathcal{B}$ as $n \rightarrow \infty$, uniformly in $t$. Finally,

$$
\left\|\mathcal{L}_{t_{n}}\left(\mathcal{L}_{t}\left(h_{n}\right)-h_{n}\right)\right\| \leq \hat{C}\left\|\mathcal{L}_{t}\left(h_{n}\right)-h_{n}\right\|_{\mathcal{B}}
$$

so that it suffices to see that $\lim _{t \downarrow 0}\left\|\mathcal{L}_{t} g-g\right\|_{\mathcal{B}}=0$ for any $\mathcal{C}^{2}$ function $g \in \mathcal{C}_{\sim}^{0}$, which is precisely what we proved above.

To apply the results of Butterley $[\mathrm{Bu}]$ to prove Theorem 1.2 , we will use the following lemma.

Lemma 4.7. There exists $C>0$ such that $\left|\mathcal{L}_{t} f-f\right|_{w} \leq C t\|f\|_{\mathcal{B}}$ for all $t \geq 0$ and all $f \in \mathcal{B}$. 
Proof. Let $f \in \mathcal{C}^{2}\left(\Omega_{0}\right)$ and fix $W \in \mathcal{W}^{s}$ and $\psi \in \mathcal{C}^{\alpha}(W)$ with $|\psi|_{\mathcal{C}^{\alpha}(W)} \leq 1$. Then using the group property as in (2.11) and changing variables,

$$
\begin{aligned}
\int_{W}\left(\mathcal{L}_{t} f-f\right) \psi d m_{W} & =\int_{W} \int_{0}^{t} \partial_{s}\left(f \circ \Phi_{-s}\right) \psi d m_{W}=\left.\int_{0}^{t} \int_{W} \partial_{r}\left(f \circ \Phi_{r}\right)\right|_{r=0} \circ \Phi_{-s} \psi d m_{W} d s \\
& =\left.\int_{0}^{t} \sum_{W_{i} \in \mathcal{G}_{s}(W)} \int_{W_{i}} \partial_{r}\left(f \circ \Phi_{r}\right)\right|_{r=0} \psi \circ \Phi_{s} J_{W_{i}} \Phi_{s} d m_{W} d s \\
& \leq t\|f\|_{0} \sum_{W_{i} \in \mathcal{G}_{s}(W)}\left|\psi \circ \Phi_{s}\right|_{\mathcal{C}^{\alpha}\left(W_{i}\right)}\left|J_{W_{i}} \Phi_{s}\right|_{\mathcal{C}^{\alpha}\left(W_{i}\right)} \leq C t\|f\|_{0},
\end{aligned}
$$

where we have used Lemma 3.6 and Lemma 3.8(b) in the last line to bound the sum independently of $s$. Now taking the supremum over $W$ and $\psi$ yields $\left|\mathcal{L}_{t} f-f\right|_{w} \leq C t\|f\|_{0}$, and taking the supremum over $f$ with $\|f\|_{\mathcal{B}} \leq 1$ proves the lemma.

5. Quasi-COMPaCtNess of the RESOlvent $\mathcal{R}(z)$, FIRST RESUlts on the SPECTRUm of $X$

In this section, we use the control over $\mathcal{L}_{t}$ established in the previous section to deduce the quasi-compactness of the resolvent acting on $\mathcal{B}$ and obtain the first spectral properties of the generator $X$. We begin by defining the generator $X$ of the semi-group acting on elements of $\mathcal{B}$,

$$
X f=\lim _{t \downarrow 0} \frac{\mathcal{L}_{t} f-f}{t} .
$$

The domain of $X$ is defined as the set of $f \in \mathcal{B}$ for which the above limit converges in the norm of $\mathcal{B}$. By Lemma 4.6, the operator $\mathcal{L}_{t}$ is strongly continuous on $\mathcal{B}$ which implies Da, Lemmas $6.1 .11,6.1 .14$ that $X$ is a closed operator, with domain dense in $\mathcal{B}$. (Indeed by Lemma 7.5, the domain of $X$ contains $\mathcal{C}^{2}\left(\Omega_{0}\right)$.) This allows us to define the resolvent $\mathcal{R}(z)=(z I d-X)^{-1}$. For $z=a+i b, a>0$, the resolvent $\mathcal{R}(z)$ has the representation,

$$
\mathcal{R}(z) f=\int_{0}^{\infty} e^{-z t} \mathcal{L}_{t} f d t
$$

and is a bounded operator on $\mathcal{B}$. By induction, the iterates of $\mathcal{R}(z)$ thus satisfy

$$
\mathcal{R}(z)^{n} f=\int_{0}^{\infty} \frac{t^{n-1}}{(n-1) !} e^{-z t} \mathcal{L}_{t} f d t .
$$

We will use repeatedly the fact that for any $z \in \mathbb{C}$ with $\operatorname{Re}(z)>0$,

$$
\left|\int_{0}^{\infty} \frac{t^{n-1}}{(n-1) !} e^{-z t} d t\right| \leq(\operatorname{Re}(z))^{-n}
$$

Recall the hyperbolicity exponents $\Lambda>1$ from (1.2) and $\lambda$ from the growth Lemma 3.8 In Subsection 5.1, we will prove the following proposition.

Proposition 5.1 (Lasota-Yorke inequalities for $\mathcal{R}(z)$ ). Recall that $\gamma \leq \min \{\alpha-\beta, 1 / q\}$. For any

$$
1>\tilde{\lambda}>\max \left\{\Lambda^{-\beta}, \Lambda^{-\gamma}, \lambda^{1-1 / q}\right\}
$$

there exists $C \geq 1$ such that for all $z \in \mathbb{C}$ with $\operatorname{Re}(z)=a>0$, all $f \in \mathcal{B}$ and all $n \geq 0$,

$$
\begin{aligned}
\left|\mathcal{R}(z)^{n} f\right|_{w} & \leq C a^{-n}|f|_{w} \\
\left\|\mathcal{R}(z)^{n} f\right\|_{s} & \leq C(a-\ln \tilde{\lambda})^{-n}\|f\|_{s}+C a^{-n}|f|_{w} \\
\left\|\mathcal{R}(z)^{n} f\right\|_{u} & \leq C(a-\ln \tilde{\lambda})^{-n}\|f\|_{u}+C a^{-n}\|f\|_{s}+C a^{-n}\|f\|_{0} \\
\left\|\mathcal{R}(z)^{n} f\right\|_{0} & \leq C a^{1-n}\left(1+a^{-1}|z|\right)|f|_{w} .
\end{aligned}
$$


From this proposition, we deduce below a bound on the essential spectral radius of $\mathcal{R}(z)$ :

Corollary 5.2 (Spectral and essential spectral radii of $\mathcal{R}(z)$ ). Let $\tilde{\lambda}<1$ and $C$ be as in Proposition 5.1. If the constant $c_{u}$ defining the norm in $\mathcal{B}$ is small enough (depending only on $C$ from Proposition [5.1) then, for any $z=a+i b$ with $a \geq 1$, the essential spectral radius of $\mathcal{R}(z)$ on $\mathcal{B}$ is bounded by $(a-\ln \tilde{\lambda})^{-1}$, while its spectral radius is bounded by $a^{-1}$.

Remark 5.3 (Optimal strip). Since our choice of homogeneity layers gives $\alpha \leq 1 / 3$, it is easy to see that the optimal choice for our parameters in view of Corollary 5.2 is

$$
\frac{1}{q}=\frac{1}{2}, \quad \alpha=\frac{1}{3}, \quad \beta=\frac{1}{6}, \quad \gamma=\frac{1}{6},
$$

giving a Banach space $\mathcal{B}$ for which we can take $\tilde{\lambda}>\Lambda^{-1 / 6}$ arbitrarily close to $\Lambda^{-1 / 6}$. By choosing homogeneity layers $k^{\chi}$ with $\chi>1$ arbitrarily close to 1 (see footnote 14), we can consider the space $\mathcal{B}$ associated to $1 / q=1 / 2, \beta=1 / 4, \alpha<1 / 2$ arbitrarily close to $1 / 2$, and $\gamma<1 / 4$ arbitrarily close to $1 / 4$, so that we can take $\tilde{\lambda}>\Lambda^{-1 / 4}$ arbitrarily close to $\Lambda^{-1 / 4}$. (This seems to be the optima 27 strip, since for a piecewise hyperbolic symplectic map or hamiltonian flow we expect $\Lambda^{-1 / 2}$ in view of [DL, Remark 5.9], and the further square root appears natural in view of the billiard singularity type, see Lemma 3.4.) To get exponential decay of correlations (Corollary 1.3), we shall need to consider in Section 9 a Banach space $\widehat{\mathcal{B}} \subset \mathcal{B}$ corresponding to $\beta>0$ very small, and $1 / q<1$ very close to 1 (we may take $\alpha \leq 1 / 3$ and take smaller $\gamma$ if needed). However, the non-essential spectra of $\mathcal{R}$ on the Banach spaces $\mathcal{B}$ and $\widehat{\mathcal{B}}$ corresponding to these different parameters coincide (see e.g. $[\mathrm{BT2}$, App. A], using that the spaces $\widehat{\mathcal{B}} \subset \mathcal{B}$ are continuously embedded in $\left(C^{1 / 4}\left(\Omega_{0}\right)\right)^{*}$, while their intersection $\widehat{\mathcal{B}}$ is a Banach space dense in $\mathcal{B}$, recalling (2.10) ), so the final spectral result also holds on the space $\mathcal{B}$ for $\frac{1}{q}=\frac{1}{2}, \alpha=\frac{1}{3}$, $\beta=\frac{1}{6}, \gamma=\frac{1}{6}$. Note finally that the choice of homogeneity layer decay also affects the constants in the approximate foliation constructed in Section 6 and used to prove Lemma 8.1 and thus Theorem 1.2 and its corollary. The choice of homogeneity layers made there is independent from the one used to get Corollary [5.2, and in any case taking $1<\chi<2$ close to 1 will in fact give a better exponent for the foliations in Section 6 and Remark 1.1 .

Proof of Corollary 5.2. Fixing $z=a+i b$ with $a>0$, we choose $n \geq 1$ minimal so that $2 C /(1-$ $\left.a^{-1} \ln \tilde{\lambda}\right)^{n}=: \nu_{a}^{n}<1$, where $C$ is from Proposition [5.1. If $a \geq 1$, we have $0<c_{u}<\left(1-a^{-1} \ln \tilde{\lambda}\right)^{-n}$, if $c_{u}>0$ satisfies $c_{u}<\left(1-a^{-1} \ln \tilde{\lambda}\right)^{-n}$, with $n$ fixed as above. Now,

$$
\begin{aligned}
a^{n}\left\|\mathcal{R}(z)^{n} f\right\|_{\mathcal{B}}= & a^{n}\left\|\mathcal{R}(z)^{n} f\right\|_{s}+c_{u} a^{n}\left\|\mathcal{R}(z)^{n} f\right\|_{u}+a^{n}\left\|\mathcal{R}(z)^{n} f\right\|_{0} \\
\leq & C\left(1-a^{-1} \ln \tilde{\lambda}\right)^{-n}\|f\|_{s}+C|f|_{w}+c_{u} C\left(1-a^{-1} \ln \tilde{\lambda}\right)^{-n}\|f\|_{u} \\
& \quad+c_{u} C\|f\|_{s}+c_{u} C\|f\|_{0}+C a\left(1+a^{-1}|z|\right)|f|_{w} \\
\leq & 2 C\left(1-a^{-1} \ln \tilde{\lambda}\right)^{-n}\left(\|f\|_{s}+c_{u}\|f\|_{u}+\|f\|_{0}\right)+C(1+|z|+a)|f|_{w} \\
\leq & \nu_{a}^{n}\|f\|_{\mathcal{B}}+C(1+|z|+a)|f|_{w} .
\end{aligned}
$$

By a classical result of Hennion $[\mathrm{H}]$, this, together with the compactness of the unit ball of $\mathcal{B}$ in $\mathcal{B}_{w}$ (Lemma 3.10), implies that the essential spectral radius of $\mathcal{R}(z)$ on $\mathcal{B}$ is bounded by $(a-\ln \tilde{\lambda})^{-1}$, while its spectral radius is bounded by $a^{-1}$.

As a consequence of Corollary [5.2, we get our first result on the spectrum of the generator $X$ (see also Corollary 9.2):

\footnotetext{
27 In particular, taking other values than the "Hilbert-space" choice $q=2$ does not help here. However, for the proof of Proposition 9.1 it is crucial to let $q$ tend to 1.
} 
Corollary 5.4 (Spectrum of $X$ ). Under the assumptions of Corollary 5.2, the following holds: The spectrum of $X$ on $\mathcal{B}$ is contained in the left half-plane $\Re z \leq 0$, and its intersection with the half-plane $\{z \in \mathcal{C} \mid \Re z>\ln \tilde{\lambda}\}$ consists of at most countably many isolated eigenvalues of finite multiplicity. In addition, the spectrum on the imaginary axis consists in a finite union of discrete additive subgroups of $\mathbb{R}$, and if $b \in \mathbb{R}$ then $X \psi=i b \psi$ for $\psi \in \mathcal{B}$ implies $\psi \in L^{\infty}$, while $X \psi=i b \psi$ for $\psi \in L^{1}$ implies $\psi \in \mathcal{B}$. Finally, $X$ has an eigenvalue of algebraic multiplicity one at 0 .

Proof. The arguments are standard, see e.g. [BL, Lemma 3.6, Cor. 3.7]. A nonzero $\rho \in \mathbb{C}$ lies in the spectrum of $\mathcal{R}(z)$ on $\mathcal{B}$ if and only if $\rho=(z-\tilde{\rho})^{-1}$, where $\tilde{\rho}$ lies in the spectrum of $X$ as a closed operator on $\mathcal{B}$ (see e.g. [Da, Lemma 8.1.9]). For such a pair $(\rho, \tilde{\rho})$, is easy to check that, for any $k \geq 1$ and any $\psi \in \mathcal{B}$, we have $(\mathcal{R}(z)-\rho)^{k}(\psi)=0$ if and only if $(X-\tilde{\rho})^{k}(\psi)=0$, so that $\rho$ is an eigenvalue of $\mathcal{R}(z)$ of algebraic multiplicity $m_{0}, 1 \leq m_{0} \leq \infty$, if and only if $\tilde{\rho}$ is an eigenvalue of $X$ of algebraic multiplicity $m_{0}$. The first two claims then follow from Corollary 5.2 .

It remains to study the spectrum on the imaginary axis. First note that if $X(\psi)=i b \psi$ for $b \neq 0$, then $\mathcal{R}(a+i b)(\psi)=a^{-1} \psi$. Another simple computation, using [Da, Theorem 6.1.16] (noting that $\tilde{\psi}_{t}=e^{i b t} \psi$ satisfies $\left.\partial_{t} \tilde{\psi}_{t}\right|_{t=s}=X\left(\tilde{\psi}_{s}\right)$ so that $\left.\mathcal{L}_{t}\left(\tilde{\psi}_{0}\right)=\tilde{\psi}_{t}\right)$ gives

$$
\psi \circ \Phi_{t}=e^{-i b t} \psi \text {. }
$$

In the following, we will use two properties of $\mathcal{B}$ : First, there exists a set $\mathcal{D} \subset L^{\infty}\left(\Omega_{0}\right)$ dense in $\mathcal{B}$ (see (2.10) and use that $\mathcal{L}_{t}$ is a contraction in $L^{\infty}$, i.e., $\left|\mathcal{L}_{t} \psi\right|_{\infty} \leq|\psi|_{\infty}$ ). Second, if $\psi \in \mathcal{B}$ and $\int \psi \varphi d m=0$ for all $\varphi \in C^{2}$, then $\psi=0$ (due to the embedding properties in Lemma 2.11).

We next show that $\psi \in L^{\infty}$ if $\psi \circ \Phi_{t}=e^{-i b t} \psi$ for $b \in \mathbb{R}$. We already observed that this implies $\mathcal{R}(a+i b)(\psi)=a^{-1} \psi$. Next, let $\left\{\zeta_{j}\right\}_{i=1}^{K}, \zeta_{1}=a^{-1}$, be the finitely many eigenvalues of modulus $a^{-1}$ of $R(z)$. By the spectral decomposition, [Ka, we can write

$$
\mathcal{R}(z)=\sum_{i=1}^{K}\left[\zeta_{i} \Pi_{i}(z)+N_{i}(z)\right]+Q(z), \quad z=a+i b,
$$

where the operators $\Pi_{i}(z)$ and $N_{i}(z)$ are finite rank, the $\Pi_{i}(z), N_{i}(z)$, and $Q(z)$ commute, with $\Pi_{i}(z)^{2}=\Pi_{i}(z), \Pi_{i}(z) Q(z)=N_{j}(z) \Pi_{i}(z)=0$, for $i \neq j, \Pi_{i}(z) N_{i}(z)=N_{i}(z)$, and, if $N_{i}(z) \neq 0$, there exists $d_{i}(z) \in \mathbb{N}$ such that $N_{i}(z)^{d_{i}(z)+1}=0$ while $N_{i}(z)^{d_{i}(z)} \neq 0$. In addition there exist $C(z)>0$ and $\rho_{0}(z)<a^{-1}$ such that $\left\|Q^{n}(z)\right\| \leq C(z) \rho_{0}(z)^{n}$. (We suppress the $z$ dependence when no confusion can arise.) We next show that $N_{i}(z) \equiv 0$ for all $i \in\{1, \ldots, K\}$. Assume otherwise, then, for any $n \geq 1$, we would have

$$
\mathcal{R}(z)^{n}=\sum_{i=1}^{K} \sum_{j=0}^{d_{i}(z)}\left(\begin{array}{l}
n \\
j
\end{array}\right) \zeta_{i}^{j-n} \Pi_{i}(z) N_{i}(z)^{j}+\mathcal{O}\left(\rho_{0}(z)^{n}\right),
$$

which, setting $d=\min _{i} d_{i}$, would immediately imply that there is $\tilde{\psi} \in \mathcal{B}$ for which $\left\|\mathcal{R}(z)^{n} \tilde{\psi}\right\| \geq$ $C(z) a^{-n} n^{d(z)}$, contradicting (5.4). Accordingly, (5.10) implies $\lim _{n \rightarrow \infty} \frac{1}{n} \sum_{k=0}^{n-1} a^{k} \mathcal{R}(z)^{k}=\Pi_{1}(z)$. Let $\psi_{0} \in \mathcal{D}$, then for $k \geq 1$, using that $\mathcal{L}_{t}$ preserves volume,

$$
\left|a^{k} \mathcal{R}(z)^{k}\left(\psi_{0}\right)\right|_{\infty} \leq a^{k} \int_{0}^{\infty} \frac{t^{k-1} e^{-a t}}{(k-1) !}\left|\psi_{0} \circ \Phi_{-t}\right|_{\infty} d t \leq\left|\psi_{0}\right|_{\infty}
$$

Hence, for each $\psi_{0} \in \mathcal{D}$ and $\varphi \in C^{\infty}$,

$$
\left|\int \Pi_{1}(z)\left(\psi_{0}\right) \cdot \varphi d m\right| \leq\left|\psi_{0}\right|_{\infty}|\varphi|_{L^{1}}
$$

This implies that $\Pi_{1}(\mathcal{D}) \subset L^{\infty}$. Since the range of $\Pi_{1}$ is finite-dimensional, it follows that $\Pi_{1}(z)$ is bounded from $\mathcal{B}$ to $L^{\infty}$, proving our claim that $\psi \in L^{\infty}$. 
We next check that if $\psi \in L^{1} \backslash\{0\}$ and $\mathcal{R}(a+i b)(\psi)=a^{-1} \psi$ for $b \in \mathbb{R}$, then $\psi \in \mathcal{B}$. Consider a sequence $\left\{\psi_{\varepsilon}\right\} \subset C^{\infty}$ converging to $\psi$ in $L^{1}$. Then the limit

$$
\lim _{n \rightarrow \infty} \frac{1}{n} \sum_{k=0}^{n-1} a^{k} \mathcal{R}(z)^{k}\left(\psi_{\epsilon}\right), \quad z=a+i b,
$$

always exists, and it is zero if $a^{-1} \notin \operatorname{sp}(\mathcal{R}(z))$, while it equals $\Pi_{1}(z)\left(\psi_{\varepsilon}\right)$ if $a^{-1} \in \operatorname{sp}(\mathcal{R}(z))$, where $\Pi_{1}(z)$ is the eigenprojector associated to $a^{-1}$. In the first case, for each $\varphi \in C^{2}$,

$$
\begin{aligned}
\left|\int \psi \varphi d m\right| & \leq \lim _{\varepsilon \rightarrow 0} \lim _{n \rightarrow \infty} \frac{1}{n} \sum_{k=0}^{n-1} a^{k} \int_{0}^{\infty} e^{-a t} \frac{t^{k-1}}{(k-1) !}\left|\psi_{\varepsilon}-\psi\right|_{L^{1}} \cdot|\varphi \circ \Phi(t)|_{\infty} d t \\
& \leq \lim _{\varepsilon \rightarrow 0}\left|\psi_{\varepsilon}-\psi\right|_{L^{1}} \cdot|\varphi|_{\infty}=0 .
\end{aligned}
$$

We would then have $\psi \equiv 0$, a contradiction. Hence $a^{-1}$ is an eigenvalue, and by the same computation as above

$$
\left|\int\left(\psi-\Pi_{1}(z)\left(\psi_{\varepsilon}\right)\right) \varphi d x\right| \leq\left|\psi_{\varepsilon}-\psi\right|_{L^{1}} \cdot|\varphi|_{\infty}
$$

Since $\Pi_{1}(z)$ is a projector, $\Pi_{1}(z)=\sum_{k} \tilde{\psi}_{k}(z)\left[\ell_{k}(z)\right](\cdot)$, where $\tilde{\psi}_{k}(z) \in \mathcal{B} \cap L^{\infty}$, the $\ell_{k}(z)$ belong to the dual of $\mathcal{B}$, and $\ell_{k}\left(\tilde{\psi}_{j}\right)=\delta_{k j}$. Then (5.13) shows that $\ell_{k}\left(\psi_{\varepsilon}\right)$ is bounded uniformly in $\epsilon$. We can then extract a subsequence $\varepsilon_{j}$ such that $\Pi_{1}(z)\left(\psi_{\varepsilon_{j}}\right)$ is convergent. Thus, $\psi$ is a linear combination of the $\tilde{\psi}_{k}$, concluding the proof that $\psi \in \mathcal{B}$.

We next use the facts about $L^{1}$ and $L^{\infty}$ to show the discrete subgroup claim. If $X\left(\psi_{k}\right)=i b_{k} \psi_{k}$, with $\psi_{k} \neq 0$ for $k \in\{1,2\}$, we have $\psi_{1}, \psi_{2} \in L^{\infty}$, and

$$
\begin{aligned}
\mathcal{R}(z)\left(\psi_{1} \psi_{2}\right) & =\int_{0}^{\infty} e^{-z t}\left(\psi_{1} \circ \Phi_{-t}\right)\left(\psi_{2} \circ \Phi_{-t}\right) d t=\psi_{1} \psi_{2} \int_{0}^{\infty} e^{-z t+i\left(b_{1}+b_{2}\right) t} d t \\
& =\left(z-i b_{1}-i b_{2}\right)^{-1} \psi_{1} \psi_{2} .
\end{aligned}
$$

Clearly, $\psi:=\psi_{1} \psi_{2} \in L^{1} \backslash\{0\}$ and $\mathcal{R}\left(a+i b_{1}+i b_{2}\right)(\psi)=a^{-1} \psi$, so that $\psi \in \mathcal{B}$. Then, (5.14) implies that either $\psi_{1} \psi_{2}=0$ or $i b_{1}+i b_{2} \in \operatorname{sp}(X)$. A similar argument applied to $\bar{\psi}_{k}$ shows that $-i b_{k} \in \operatorname{sp}(X)$. Thus $\left|\bar{\psi}_{k}\right|^{2}$ belongs to the finite dimensional eigenspace of the eigenvalue zero and $\left\{i m b_{k}\right\}_{m \in \mathbb{Z}} \subset \operatorname{sp}(X)$. This ends the proof of the subgroup claim.

Finally, if $A$ is a positive measure invariant set, then $\operatorname{Id}_{A} \in L^{\infty}$ (which belongs to $\mathcal{B}$ by the argument above) is an eigenvector associated to zero, and if $\psi$ is an eigenvector associated to zero (we can assume without loss of generality that $\psi$ is real) then $\{\psi \geq \lambda\}$ are invariant sets, that is, $\psi$ must be piecewise constant (otherwise zero would have infinite multiplicity). In other words the eigenspace of zero is spanned by the characteristic functions of the ergodic decomposition of Lebesgue. By ergodicity of the billiard flow, zero is a simple eigenvalue.

5.1. Norm estimates for $\mathcal{R}(z)$. In this subsection, we prove Proposition [5.1, Fix $1>\tilde{\lambda}>$ $\max \left\{\Lambda^{-\beta}, \Lambda^{-\gamma}, \lambda^{(1-1 / q)}\right\}$.

Now taking $W \in \mathcal{W}^{s}$ and $\psi \in \mathcal{C}^{\alpha}(W)$ with $|\psi|_{\mathcal{C}^{\alpha}(W)} \leq 1$, we have by (4.8) and (5.3),

$$
\left|\int_{W} \mathcal{R}(z)^{n} f \psi d m_{W}\right|=\left|\int_{0}^{\infty} \int_{W} \mathcal{L}_{t} f \psi d m_{W} \frac{t^{n-1}}{(n-1) !} e^{-z t} d t\right| \leq C|f|_{w} a^{-n},
$$

which proves (5.4). 
Similarly, taking $\psi \in \mathcal{C}^{\beta}(W)$ with $|W|^{1 / q}|\psi|_{\mathcal{C}^{\beta}(W)} \leq 1$, we have by (4.15),

$$
\begin{aligned}
\left|\int_{W} \mathcal{R}(z)^{n} f \psi d m_{W}\right| & =\left|\int_{0}^{\infty} \int_{W} \mathcal{L}_{t} f \psi d m_{W} \frac{t^{n-1}}{(n-1) !} e^{-z t} d t\right| \\
& \leq \int_{0}^{\infty}\left[C\left(\Lambda^{-t q}+\lambda^{t(1-1 / q)}\right)\|f\|_{s}+C|f|_{w}\right] \frac{t^{n-1}}{(n-1) !} e^{-a t} d t \\
& \leq C(a-\ln \tilde{\lambda})^{-n}\|f\|_{s}+C a^{-n}|f|_{w},
\end{aligned}
$$

where we have again used (5.3), proving (5.5).

To prove (5.6), fixing $\varepsilon>0$ and taking $W_{1}, W_{2}$ with $d_{\mathcal{W}^{s}}\left(W_{1}, W_{2}\right)<\varepsilon$, and $\psi_{i} \in \mathcal{C}^{\alpha}\left(W_{i}\right)$ with $\left|\psi_{i}\right|_{\mathcal{C}^{\alpha}\left(W_{i}\right)} \leq 1$ and $d\left(\psi_{1}, \psi_{2}\right)=0$, we estimate

$$
\begin{array}{rl}
\varepsilon^{-\gamma} \mid \int_{W_{1}} & \mathcal{R}(z)^{n} f \psi_{1} d m_{W}-\int_{W_{2}} \mathcal{R}(z)^{n} f \psi_{2} d m_{W} \mid \\
= & \left|\int_{0}^{\infty} \frac{t^{n-1}}{(n-1) !} e^{-z t} \varepsilon^{-\gamma}\left(\int_{W_{1}} \mathcal{L}_{t} f \psi_{1} d m_{W}-\int_{W_{2}} \mathcal{L}_{t} f \psi_{2} d m_{W}\right) d t\right| \\
\leq & \int_{0}^{\infty} \frac{t^{n-1}}{(n-1) !} e^{-a t}\left(C \tilde{\lambda}^{t}\|f\|_{u}+C\left(\|f\|_{0}+\|f\|_{s}\right)\right) d t \\
\leq & C(a-\ln \tilde{\lambda})^{-n}\|f\|_{u}+C a^{-n}\left(\|f\|_{s}+\|f\|_{0}\right),
\end{array}
$$

where we have used (5.3) and (4.4). Taking the appropriate suprema proves (5.6).

We prove the neutral norm estimate (5.7). Let $W \in \mathcal{W}^{s}$ and $\psi \in \mathcal{C}^{\alpha}(W)$ with $|\psi|_{\mathcal{C}^{\alpha}(W)} \leq 1$. Using the definition of the neutral norm, we write for $f \in \mathcal{B} \cap \mathcal{C}^{1}\left(\Omega_{0}\right)$,

$$
\begin{aligned}
\left.\int_{W} \partial_{s}\left(\left(\mathcal{R}(z)^{n} f\right) \circ \Phi_{s}\right)\right|_{s=0} \psi d m_{W} & =\left.\int_{W} \int_{0}^{\infty} \frac{t^{n-1}}{(n-1) !} e^{-z t} \partial_{s}\left(\left(\mathcal{L}_{t} f\right) \circ \Phi_{s}\right)\right|_{s=0} d t \psi d m_{W} \\
& =\left.\int_{W} \int_{0}^{\infty} \frac{t^{n-1}}{(n-1) !} e^{-z t} \partial_{s}\left(f \circ \Phi_{-s}\right)\right|_{s=t} d t \psi d m_{W} \\
& =-\int_{0}^{\infty}\left(\frac{t^{n-2}}{(n-2) !}-\frac{z t^{n-1}}{(n-1) !}\right) e^{-z t} \int_{W} \mathcal{L}_{t} f \psi d m_{W} d t,
\end{aligned}
$$

where in the last line we have integrated by parts and used the fact that $\mathcal{L}_{t} f=f \circ \Phi_{-t}$. Finally, recalling the weak norm estimate (4.8) for the integral on $W$ and using (5.3) to integrate with respect to $d t$ yields (5.7). This ends the proof of Proposition 5.1.

\section{Construction of approximate unstable Lipschitz foliations}

Fix a length scale $0<\rho<L_{0}$. For any homogeneous flow stable curve $W \in \mathcal{W}^{s}$ such that

$$
\text { (a) } d\left(W, \partial \Omega_{0}\right) \geq 2 C_{d} \rho \text { and }(b) \quad P^{+}(W) \subset \mathbb{H}_{k_{W}},
$$

where $C_{d}>1$ is defined by (6.1) and $k_{W}$ satisfies $k_{W} \leq C \rho^{-1 / 5}$, and for any $\varkappa>0$ and any $x^{0}$ such that $\Phi_{t}(W)$ remains at least $C_{d} \rho$ away from a collision for all $|t| \leq\left|x^{0}\right|$, we shall construct in Theorem 6.2 below a surface $\mathcal{F}_{\varkappa, x^{0}}$ containing $\Phi_{x^{0}}(W)$ and transversal to the flow, and a transverse foliation of a two-dimensional neighborhood of $W$ in $\mathcal{F}_{\varkappa, x^{0}}$ by (one-dimensional) curves $\gamma$ so that each $\gamma$ is a flow-unstable curve, and so that $\Phi_{-t}(\gamma)$ is an unstable curve for $0 \leq t<\varkappa$ and "most" curves $\gamma$.

But first, we need to define good local coordinates (making precise Remark 2.4):

28 The exponent $-1 / 5$ comes from our choice of homogeneity layer (the length of the fiber, $k_{W}^{2} \rho$ must be less than the width of the strip $k_{W}^{-3}$ ); this choice will impact the exponents throughout this section, without further notice. 
Remark 6.1 ( $\mathcal{C}^{2}$ cone-compatible Darboux charts). Let $W$ satisfy (a) and (b) above. For any $Z \in W$, by (b) we have $\cos \varphi\left(P^{+}(Z)\right) \geq C \rho^{2 / 5}$ for some $C>0$. Similarly, if $Z^{\prime} \in \Omega_{0}$ satisfies $d\left(Z, Z^{\prime}\right)<C_{d} \rho$, we have $\tau\left(Z^{\prime}\right) \geq C_{d} \rho$ and $\cos \varphi\left(P^{+}\left(Z^{\prime}\right)\right) \geq C \rho^{2 / 5}$ since $P^{+}\left(Z^{\prime}\right)$ lies either in $\mathbb{H}_{k_{W}}$ or $\mathbb{H}_{k_{W} \pm 1}$. Thus it follows from (2.4) that the width of the stable cones at $Z$ and $Z^{\prime}$ has angle at least of order $\rho^{2 / 5}$, and the maximum and minimum slopes in $C^{s}(Z)$ and $C^{s}\left(Z^{\prime}\right)$ are uniformly bounded multiples of one another. Similar considerations hold for the unstable cones. Thus, fixing $Z \in W$, we may adopt local coordinates $\left(x^{u}, x^{s}, x^{0}\right)$ in a $c_{1} \rho$ neighborhood of $Z$, as introduced in Remark 2.4, for which the contact form is in standard form and $Z$ is the origin. $B y$ the above discussion, the charts effecting this change of coordinates are uniformly $\mathcal{C}^{2}$. The constant $c_{1} \geq 1$ is chosen large enough that the box $\left\{\left(x^{u}, x^{s}, x^{0}\right): x^{s} \in W,\left|x^{u}\right| \leq 2 \rho,\left|x^{0}\right| \leq 2 \rho\right\}$ is well-defined in these new coordinates, and if necessary, $C_{d}$ is increased accordingly.

Adopting such local coordinates $x^{u}, x^{s}$, and $x^{0}$ such that $W=\left\{\left(0, x^{s}, 0\right): x^{s} \in[0,|W|]\right\}$, for each fixed $0<\varpi \leq 1 / 35$ and $\varkappa>0$, and each $x^{0}$ so that $d\left(\Phi_{t}(W), \partial \Omega_{0}\right) \geq C_{d} \rho$ for all $|t| \leq\left|x^{0}\right|$, Theorem 6.2 will provide a surface

$$
\mathcal{F}=\left\{\mathbb{F}\left(x^{u}, x^{s}\right)=\left(x^{u}, G\left(x^{u}, x^{s}\right), H\left(x^{u}, x^{s}\right)+x^{0}\right): x^{s} \in[0,|W|], x^{u} \in[-\rho, \rho]\right\}
$$

transversal to the flow $\Phi_{s}$ and so that the curves $x^{u} \mapsto \gamma_{x^{s}}\left(x^{u}\right)=\left(x^{u}, G\left(x^{u}, x^{s}\right), H\left(x^{u}, x^{s}\right)+x^{0}\right)$ are pairwise disjoint, satisfying additional requirements. (We write $\gamma \in \mathcal{F}$ and view $\mathcal{F}$ as foliated by the curves $\gamma=\gamma_{x^{s}}$; in particular, $\forall Z=\left(0, x^{s}, 0\right) \in W$, the curve $\gamma_{Z}:=\gamma_{x^{s}}$ is the unique $\gamma \in \mathcal{F}$ such that $\gamma \cap W=\{Z\}$.)

Theorem 6.2. Assuming conditions (a) and (b) above, for each fixed $0<\varpi \leq 1 / 35$ and $\varkappa>0$, and each $x^{0}$ so that $d\left(\Phi_{t}(W), \partial \Omega_{0}\right) \geq C_{d} \rho$ for all $|t| \leq\left|x^{0}\right|$, it is possible to construct $\mathcal{F}=\mathcal{F}_{\varkappa, x^{0}}$ so that it satisfies the following conditions, for a constant $C$ depending on $\varpi$, but independent of $\rho, \varkappa, x^{0}$, and $W$ :

(i) $\partial_{x^{u}} H=G$, which implies $\boldsymbol{\alpha}\left(\partial_{x^{u}} \mathbb{F}\right)=0$, i.e., each $\gamma \in \mathcal{F}$ lies in the kernel of the contact form $\boldsymbol{\alpha}$. In addition, each $\gamma \in \mathcal{F}$ is an unstable curve (in particular, $\left(1, \partial_{x^{u}} G, \partial_{x^{u}} H\right) \in$ $C^{u}$ and $\left.|\gamma| \leq L_{0}\right)$ with $|\gamma| \geq \rho$.

(ii) For all $x^{s} \in[0,|W|]$ we have $G\left(0, x^{s}\right)=x^{s}$ and $H\left(0, x^{s}\right)=0$.

(iii) Let $\Delta_{\varkappa}$ denote the set of $Z \in W$ such that the $\varkappa$-trace of $\Phi_{-\varkappa}\left(\gamma_{Z}\right)$ contains no intersections with the boundaries of homogeneity strips or tangential collisions. Then for any $Z \in \Delta_{\varkappa}$, we have $\Phi_{-s}\left(\gamma_{Z}\right) \in \mathcal{W}^{u}$ for all $0 \leq s \leq \varkappa$.

(iv) $m_{W}\left(W \backslash \Delta_{\varkappa}\right) \leq C \rho$.

(v) $C^{-1} \leq\left\|\partial_{x^{s}} G\right\| \leq C$, which implies $\left|\partial_{x^{s}} \partial_{x^{u}} H\right|_{\infty} \leq C$ by (i).

(vi) $\partial_{x^{u}} \partial_{x^{s}} G \in \mathcal{C}^{0}$ and $\left|\partial_{x^{u}} \partial_{x^{s}} G\right|_{\infty} \leq C \rho^{-4 / 5}$.

(vii) The following four-point estimate holds:

$$
\begin{aligned}
\mid \partial_{x^{s}} G\left(x^{u}, x^{s}\right)-\partial_{x^{s}} G\left(x^{u}, y^{s}\right) & -\partial_{x^{s}} G\left(y^{u}, x^{s}\right)+\partial_{x^{s}} G\left(y^{u}, y^{s}\right) \mid \\
& \leq C \rho^{-4 / 5-11 \varpi / 15}\left|x^{u}-y^{u}\right|^{1-7 \varpi}\left|x^{s}-y^{s}\right|^{\varpi} .
\end{aligned}
$$

(viii) $\left|\partial_{x^{s}} H\right|_{\mathcal{C}^{0}} \leq C \rho$ and $\left|\partial_{x^{s}} H\right|_{\mathcal{C}^{\varpi}} \leq C \rho^{6 / 5-116 \varpi / 15}$.

The rest of the section is devoted to the proof of the above Theorem. We will first (Subsections 6.1 6.4) construct a foliation with the (analogue of the) above properties in a neighborhood of $P^{+}(W)$ in the phase space $\mathcal{M}$ of the billiard map and then (Subsection 6.5) describe how to lift it to $W$ to get a foliation for the billiard flow. We denote analogous objects for the map-foliation by $\overline{\mathbb{F}}, \bar{G}, \bar{\Delta}_{\varkappa}$, etc.

Before carrying out this construction, we mention a remark which will be useful below: 
Remark 6.3 (Taking the exponential of a four-point estimate). We claim that if (ii), (v), and (vi) hold, then to show (vii) it is enough to prove (vii) for the function $\log \partial_{s} G$ (up to changing the constant factor). Indeed, using (ii), first observe that (vii) for $\log \partial_{x^{s}} G$ at $x^{u}=0$ gives

$$
\begin{aligned}
\left|\log \partial_{x^{s}} G\left(y^{u}, x^{s}\right)-\log \partial_{x^{s}} G\left(y^{u}, y^{s}\right)\right| & \leq C^{2} \rho^{-4 / 5-11 \varpi / 15}\left|y^{u}\right|^{1-7 \varpi}\left|x^{s}-y^{s}\right|^{\varpi} \\
& \leq 2 C^{2} \rho^{1 / 5-116 \varpi / 15)}\left|x^{s}-y^{s}\right|^{\varpi}
\end{aligned}
$$

since $\left|y^{u}\right| \leq \rho$. Then note that for any $C_{\infty}>1$, there is a constant $C^{\prime}$ depending only on $C_{\infty}$ so that, if a function $g\left(x^{u}, x^{s}\right)$ satisfies $|g|_{\infty} \leq C_{\infty}$, and

$$
\sup _{x^{u}}\left|g\left(x^{u}, x^{s}\right)-g\left(x^{u}, y^{s}\right)\right| \leq C_{s}\left|x^{s}-y^{s}\right|^{\varpi}, \sup _{x^{s}}\left|g\left(x^{u}, x^{s}\right)-g\left(y^{u}, x^{s}\right)\right| \leq C_{u}\left|x^{u}-y^{u}\right|,
$$

and

$$
\left|g\left(x^{u}, x^{s}\right)-g\left(x^{u}, y^{s}\right)-g\left(y^{u}, x^{s}\right)+g\left(y^{u}, y^{s}\right)\right| \leq C_{u s}\left|x^{u}-y^{u}\right|^{1-7 \varpi}\left|x^{s}-y^{s}\right|^{\varpi},
$$

with $C_{u} C_{s} \leq C_{\infty} C_{u s}$, then

$$
\left|\exp g\left(x^{u}, x^{s}\right)-\exp g\left(x^{u}, y^{s}\right)-\exp g\left(y^{u}, x^{s}\right)+\exp g\left(y^{u}, y^{s}\right)\right| \leq C^{\prime} C_{u s}\left|x^{u}-y^{u}\right|^{1-7 \varpi}\left|x^{s}-y^{s}\right|^{\varpi} .
$$

Indeed, first write $\exp g=\sum_{k=0}^{\infty} g^{k} /(k !)$. Then note that an easy induction gives $\mid g\left(x^{u}, x^{s}\right)^{k}-$ $g\left(x^{u}, y^{s}\right)^{k}\left|\leq 2^{k-1} C_{\infty}^{k-1} C_{s}\right| x^{s}-\left.y^{s}\right|^{\varpi}$ and $\left|g\left(x^{u}, x^{s}\right)^{k}-g\left(y^{u}, x^{s}\right)^{k}\right| \leq 2^{k-1} C_{\infty}^{k-1} C_{u}\left|x^{u}-y^{u}\right|$ for all $k \geq 1$. Finally, the trivial formula

$a a^{\prime}-b b^{\prime}-c c^{\prime}+d d^{\prime}=c\left(a^{\prime}-b^{\prime}-c^{\prime}+d^{\prime}\right)+(a-b-c+d) d^{\prime}+(a-c)\left(a^{\prime}-b^{\prime}\right)+(a-b)\left(b^{\prime}-d^{\prime}\right)$, implies

$$
\left|g\left(x^{u}, x^{s}\right)^{k}-g\left(x^{u}, y^{s}\right)^{k}-g\left(y^{u}, x^{s}\right)^{k}+g\left(y^{u}, y^{s}\right)^{k}\right| \leq 2^{k-1} C_{\infty}^{k-1} C_{u s}\left|x^{u}-y^{u}\right|^{1-7 \varpi}\left|x^{s}-y^{s}\right|^{\varpi},
$$

for all $k \geq 1$. To conclude, use $\sum_{k \geq 0} 2^{k-1} C_{\infty}^{k-1} / k !=\left(2 C_{\infty}\right)^{-1} e^{2 C_{\infty}}$.

Now apply this bound to $g=\log \partial_{x^{s}} G$ with $C_{\infty}$ derived from (v), $C_{s}=C^{ \pm 1} \rho^{1 / 5-116 \varpi / 15}, C_{u}=$ $C^{ \pm 1} \rho^{-4 / 5}$ from (vi), and $C_{u s}=C^{ \pm 1} \rho^{-4 / 5-11 \varpi / 15}$. Then $C_{u} C_{s} \leq C_{\infty} C_{u s}$ whenever $\varpi \leq 1 / 35$.

6.1. Constructing the regular part of the map-foliation, (iii-iv). We construct the analogue $\bar{\Delta}_{\varkappa}$ for $P^{+}(W)$ of the domain $\Delta_{\varkappa}$ by putting a transverse foliation on curves $P^{+}\left(W_{i}\right)$ for $W_{i} \in \mathcal{G}_{\varkappa}(W)$ (recall Definition 3.1) and pushing it forward to $P^{+}(W)$. Let $W_{i} \in \mathcal{G}_{\varkappa}(W)$. This fixes $n=n\left(W_{i}\right)$ such that $T^{n}\left(P^{+}\left(W_{i}\right)\right) \subset P^{+}(W)$.

Using (a), we choose a uniformly $\mathcal{C}^{2}$ foliation of curves $\{\ell\}$ lying in the unstable cone for the map and transverse to $P^{+}\left(W_{i}\right)$ of length $|\ell|=k_{W}^{2} \rho /\left|J_{\ell}^{u} T^{n}\left(z_{\ell}\right)\right|$, where $z_{\ell}=P^{+}\left(W_{i}\right) \cap \ell, J_{\ell}^{u} T^{n}$ is the (unstable) Jacobian along $\ell$, and $k_{W}$ is the index of the homogeneity strip containing $P^{+}(W)$. We call this foliation $\left\{\ell_{z}\right\}_{z \in P^{+}\left(W_{i}\right)}$ a seeding foliation. Those curves $\ell$ on which $T^{n}$ is smooth (in particular, avoiding intersections with the boundaries of homogeneity strips) create a transverse foliation of map-unstable curves $\bar{\gamma}=T^{n}(\ell)$ over the subset $\bar{\Delta}_{\varkappa}$ of $P^{+}(W)$ such that $T^{j}(\ell)$ is a homogeneous unstable curve for each $j=0,1, \ldots, n$. We now estimate the size of $\bar{\Delta}_{\varkappa}$ in $P^{+}(W)$.

If $T^{j}(\ell)$ is a single homogeneous curve, then it has length

$$
\left|T^{j}(\ell)\right|=\tilde{C}_{d}^{ \pm 1} k_{W}^{2} \rho / J_{T^{j}(\ell)}^{u} T^{n-j}\left(T^{j}\left(z_{\ell}\right)\right),
$$

where

$$
\tilde{C}_{d}(j)=1+C_{d} \sup _{x, y} d\left(T^{j}(x), T^{j}(y)\right)^{1 / 3},
$$

with $C_{d}$ is the distortion constant from Lemma 3.5. Slightly abusing notation, we replace $C_{d}$ by $\max \left\{C_{d}, \max _{j} \tilde{C}_{d}(j)\right\}$, observing that $\sup _{x, y, j} d\left(T^{j}(x), T^{j}(y)\right)^{1 / 3}$ is bounded uniformly in $\rho, W$ and $\varkappa$. Consequently, $|\bar{\gamma}|=\left|T^{n}(\ell)\right|=C_{d}^{ \pm 1} k_{W}^{2} \rho$.

We note that by property (b) above regarding the homogeneity strip into which $W$ is allowed to project, we have $k_{W}^{2} \rho \leq C k_{W}^{-3}$, so that there is no need to cut any curves $T^{n} \ell$ that have not already 
been cut previously at time $1, \ldots n-1$, since they will automatically lie in a bounded number of homogeneity strips and so enjoy bounded distortion with a (properly adjusted) distortion constant $C_{d}$. Since the expansion in one step at $x$ is proportional to the reciprocal of $\cos \varphi(T x)$, and this in turn is proportional to the square of the index of the homogeneity strip containing $T(x)$ (see [CM, eq. (4.20)]), it follows that the unstable Jacobian at the last step is proportional to $k_{W}^{2}$, in other words

$$
k_{W}^{2} / J_{T^{n-1} \ell}^{u} T\left(T^{n-1} z_{\ell}\right) \leq C_{d} .
$$

The set of cuts appearing in forward iterates of $\ell$ is the extended singularity set $\mathcal{S}_{n}^{\mathbb{H}}$ from Definition 2.6. The growth lemma from [CM] we shall apply below estimates the measure of the subset of $W_{i}$ for which the corresponding $\ell$ are cut by considering the iterate $j$ at which the cut is made, and estimating the length of $T^{j}\left(W_{i}\right)$ that has had its $T^{j}(\ell)$ cut. For this, we claim that we need only look at the sets $\mathcal{S}_{1}, \mathcal{S}_{-1}$, and the boundaries of the homogeneity strips (and not any of their iterates): Notice that if a curve $S$ is in $\mathcal{S}_{1} \backslash \mathcal{S}_{0}$, it has a negative slope (is a stable curve). Its image under $T$, if it does not belong to $\mathcal{S}_{0}$, belongs to $\mathcal{S}_{-1} \backslash \mathcal{S}_{0}$ and so has a positive slope (is an unstable curve). There are exactly two "images" of curves in $\mathcal{S}_{1} \backslash \mathcal{S}_{0}$, one coming from each "side" of the curve which goes to a different scatterer.

We make cuts at time $j$ when either $(1) T^{j}(\ell)$ crosses the boundary of a homogeneity strip; or $(2) T^{j-1}(\ell)$ crosses a curve in $\mathcal{S}_{1}$ whose image belongs to $\mathcal{S}_{-1} \backslash \mathcal{S}_{0}$. (These are the only two possible cases: If $T^{j-1}(\ell)$ crosses a curve in $\mathcal{S}_{1}$ whose image belongs to $\mathcal{S}_{0}$, then in fact $T^{j}(\ell)$ will cross the boundary of a homogeneity strip; and this is case (1).)

For (1), the boundary of the homogeneity strip is horizontal (in the global $(r, \varphi)$ coordinates), so the stable curve $T^{j}\left(W_{i}\right)$ is uniformly transverse to it. Thus the length of $T^{j}\left(W_{i}\right)$ that has its $T^{j}(\ell)$ cut is at most a constant times the length of $T^{j}(\ell)$. Here it is important that $T^{j}\left(W_{i}\right)$ is transverse to the boundary of the homogeneity strips and not whether $T^{j}(\ell)$ is, although in fact, they both are.

For (2), although $\mathcal{S}_{1}$ is made of stable curves (with negative slope), we do not cut $T^{j-1}(\ell)$ when it lands across this curve (note that at time $j-1$ the derivatives are still comparable along $T^{j-1}(\ell)$ - there is no problem with smoothness or distortion at this step). We cut at the next step, when $T^{j-1}(\ell)$ maps to $T^{j}(\ell)$, which now has two (or more) connected components. The subset of $T^{j}\left(W_{i}\right)$ which is missing part of its foliation $\left\{T^{j}(\ell)\right\}$ is the subset of $T^{j}\left(W_{i}\right)$ whose $T^{j}(\ell)$ intersects this singularity curve in $\mathcal{S}_{-1}$ (the image of the curve in $\mathcal{S}_{1}$ at time $j-1$ ). Again, it is the uniform transversality of $\mathcal{S}_{-1} \backslash \mathcal{S}_{0}$ with the stable curve $T^{j}\left(W_{i}\right)$ that guarantees that the length of $T^{j}\left(W_{i}\right)$ that has had its $T^{j}(\ell)$ cut is at most a constant times the length of $T^{j}(\ell)$.

Thus, there exists a constant $C_{0}$, depending only on $C_{d}$ and the uniform transversality of stable curves and extended singularities (as explained above), such that if the intersection of $T^{j}(\ell)$ and $T^{j}\left(P^{+}\left(W_{i}\right)\right)$ lies at a distance at least $C_{0} \rho / J_{T^{j} \ell^{u}} T^{n-j-1}\left(T^{j} z_{\ell}\right)$ from the endpoints of $T^{j}\left(P^{+}\left(W_{i}\right)\right)$, then $T^{j}(\ell)$ is not cut at time $j, j=0,1, \ldots n-1$. (Recall that we do not cut at the last time step $n$ when we arrive back at $P^{+}(W)$ because we assumed that $P^{+}(W)$ is already a homogeneous stable curve.)

Setting $i=n-j$ and letting $r_{-i}(z)$ denote the distance from $T^{-i}(z)$ to the nearest endpoint of the connected homogeneous components of $T^{-i}(W)$ belonging to $\mathcal{G}_{i}(W)$, we conclude that if $T^{n-i}(\ell)$ is cut at time $i$, then $r_{-i}(z) \leq C_{0} \rho \Lambda_{0}^{-i+1}$, where $z$ is the point of intersection of $T^{n-i}(\ell)$ and $T^{-i}(W)$, and $\Lambda_{0}>1$ is defined above (1.2). Thus

$$
P^{+}(W) \backslash \bar{\Delta}_{\varkappa} \subseteq \bigcup_{i=0}^{n-1}\left\{z \in P^{+}(W): r_{-i}(z) \leq C_{0} \rho \Lambda^{-i+1}\right\} .
$$


Using the growth lemma [CM, Theorem 5.52 and Exercise 5.49], there exist constants $C>0$, $\lambda_{0}<1$, such that for all $\varepsilon>0$,

$$
\begin{aligned}
m_{P^{+}(W)}\left(r_{-i}(z)<\varepsilon\right) & \leq C\left(\lambda_{0} \Lambda_{0}\right)^{i} m_{P^{+}(W)}\left(r_{0}(z)<\varepsilon \Lambda_{0}^{-i}\right)+C \varepsilon\left|P^{+}(W)\right| \\
& \leq 2 C \varepsilon \lambda_{0}^{i}+C \varepsilon\left|P^{+}(W)\right| .
\end{aligned}
$$

Applying this bound with $\varepsilon=C_{0} \rho \Lambda_{0}^{-i+1}$, and summing over $i$, we estimate,

$$
m_{P^{+}(W)}\left(P^{+}(W) \backslash \bar{\Delta}_{\varkappa}\right) \leq \sum_{i=0}^{n-1} C^{\prime} \rho \Lambda_{0}^{-i+1} \lambda_{0}^{i}+C^{\prime} \rho \Lambda_{0}^{-i+1}\left|P^{+}(W)\right| \leq C^{\prime \prime} \rho .
$$

This implies the analogue for $\bar{\Delta}_{\varkappa}$ of item (iv) of the foliation.

6.2. Smoothness of the regular part of the map-foliation, $(\mathbf{v}-\mathbf{v i}-\mathbf{v i i})$. We proceed to check the regularity of the parts of the foliation $\left\{\ell_{z}\right\}_{z \in T^{-n} \bar{\Delta}_{x}}$ which survive uncut until time $n$.

Let $\bar{W}_{i} \subset P^{+}\left(W_{i}\right)$ denote the maximal subcurve of $P^{+}\left(W_{i}\right)$ so that $\left\{T^{n} \ell_{z}\right\}_{z \in \bar{W}_{i}}=\left\{\bar{\gamma}_{x}\right\}_{x \in T^{n} \bar{W}_{i}}$ is not cut. Let $k_{j}$ denote the index of the homogeneity strip containing $T^{n-j}\left(\bar{W}_{i}\right)$. Since the foliation is not cut at step $j$ and has length given by (6.1), there exists $C>0$ (depending only on distortion and uniform transversality of the unstable cone with the boundaries of homogeneity strips) such that for any $\ell$ so that $z_{\ell} \in \bar{W}_{i}$,

$$
\frac{k_{W}^{2} \rho}{J_{T^{n-j} \ell}^{u} T^{j}\left(T^{n-j} z_{\ell}\right)} \leq C k_{j}^{-3} \Longrightarrow k_{j}^{2} \leq\left(\frac{C J_{T^{n-j} \ell}^{u} T^{j}\left(T^{n-j} z_{\ell}\right)}{k_{W}^{2} \rho}\right)^{2 / 3} .
$$

Now let $V_{0}=T^{n}\left(\bar{W}_{i}\right) \subset P^{+}(W)$ and let $V$ denote a map-stable curve such that the surviving foliation $\{\bar{\gamma}\}$ associated to $\bar{W}_{i}$ via $V_{0}$, intersects every point of $V$. Let $\mathbf{h}_{V}: V_{0} \rightarrow V$ denote the holonomy map defined by the foliation $\{\bar{\gamma}\}$.

Set $V^{n}=T^{-n} V$ and note that since the surviving foliation intersects every point of $V$, it follows that $V^{n}$ lies in the same homogeneity strip as $\bar{W}_{i}$ and the seeding foliation of unstable curves $\{\ell\}$ intersects every point of $V^{n}$. Let $\mathbf{h}_{V^{n}}$ denote the holonomy map from $\bar{W}_{i}$ to $V^{n}$.

The lemma below is a refinement 29 of [CM, Theorem 5.42] for the Jacobian $J \mathbf{h}_{V}$. (We shall use it below to obtain claim (vi), after relating the Jacobian with $\partial_{x^{s}} G$ in appropriate coordinates.)

Lemma 6.4 (Bounds on the Jacobian outside of the gaps). There exists $C>0$, independent of $W, \rho$, and $\varkappa$, such that for any $x \in V_{0}$,

$$
\left|\ln J \mathbf{h}_{V}(x)\right|=\left|\ln \left(\frac{J_{V_{0}}^{s} T^{-n}(x)}{J_{V}^{s} T^{-n}(\tilde{x})} J \mathbf{h}_{V^{n}}\left(T^{-n} x\right)\right)\right| \leq C\left(d(x, \tilde{x}) \rho^{-2 / 3} k_{W}^{-2}+\phi_{V}(x, \tilde{x})\right) .
$$

where $\tilde{x}=\mathbf{h}_{V}(x), J_{V}^{s} T^{-n}$ denotes the (stable) Jacobian of $T^{-n}$ along $V$, and $\phi_{V}(x, \tilde{x})$ is the angle between the tangent vector to $V_{0}$ at $x$ and the tangent vector to $V$ at $\tilde{x}$.

Proof. We begin by writing,

$$
\ln J \mathbf{h}_{V}(x)=\ln J \mathbf{h}_{V^{n}}\left(x_{n}\right)+\sum_{j=0}^{n-1} \ln J_{T^{-j} V_{0}}^{s} T^{-1}\left(x_{j}\right)-\ln J_{T^{-j} V^{s}}^{T^{-1}}\left(\bar{x}_{j}\right),
$$

where $x_{j}=T^{-j}(x)$ and $\tilde{x}_{j}=T^{-j}(\tilde{x}), j=0,1, \ldots n$.

29 The analogous time reversed counterpart from [CM] would be $\left|\ln J \mathbf{h}_{V}(x)\right| \leq C\left(d(x, \tilde{x})^{1 / 3}+\phi_{V}(x, \tilde{x})\right)$. Here, we leverage the fact that we require the transverse foliation to survive for only $n$ steps (not for all time) in addition to the fact that the surviving curves must be long in the length scale $\rho$, to improve the exponent of $d(x, \tilde{x})$ to 1 , at the cost of having the constant depend on $\rho$. 
Letting $x_{j}=\left(r_{j}, \varphi_{j}\right), \mathcal{K}_{j}=\mathcal{K}\left(r_{j}\right), \tau_{j}=\tau\left(x_{j}\right)$, and similarly for $\tilde{\varphi}_{j}, \widetilde{\mathcal{K}}_{j}$ and $\widetilde{\tau}_{j}$, we have the time reversal counterpart to [CM, eq. (5.24)],

$\ln J_{T^{-j} V_{0}}^{S} T^{-1}\left(x_{j}\right)=-\ln \cos \varphi_{j+1}+\ln \left(\cos \varphi_{j}+\tau_{j+1}\left(\mathcal{K}_{j}+\left|\mathcal{V}_{j}\right|\right)\right)+\frac{1}{2} \ln \left(1+\mathcal{V}_{j+1}^{2}\right)-\frac{1}{2} \ln \left(1+\mathcal{V}_{j}^{2}\right)$,

where $\mathcal{V}_{j}<0$ represents the slope of the tangent vector to $T^{-j}\left(V_{0}\right)$ at $x_{j}$. Using the analogous expression for $\ln J_{T^{-j} V^{S}}^{T^{-1}}\left(\tilde{x}_{j}\right)$ and letting $\ell_{n}$ denote the curve of the initial seeding foliation containing $x_{n}$, we first compare,

$$
\begin{aligned}
\left|\ln \cos \varphi_{j+1}-\ln \cos \tilde{\varphi}_{j+1}\right| & \leq C \frac{\left|\varphi_{j+1}-\tilde{\varphi}_{j+1}\right|}{\cos \varphi_{j+1}} \leq C d\left(x_{j+1}, \tilde{x}_{j+1}\right) k_{j+1}^{2} \\
& \leq C \frac{d(x, \tilde{x})}{J_{T^{n-j-1} \ell_{n}}^{u} T^{j+1}\left(x_{j+1}\right)} \frac{\left(J_{T^{n-j-1} \ell_{n}}^{u} T^{j+1}\left(x_{j+1}\right)\right)^{2 / 3}}{k_{W}^{4 / 3} \rho^{2 / 3}} \\
& \leq C \frac{d(x, \tilde{x})}{k_{W}^{4 / 3} \rho^{2 / 3}\left(J_{T^{n-j-1} \ell_{n}}^{u} T^{j+1}\left(x_{j+1}\right)\right)^{1 / 3}} \leq C \frac{d(x, \tilde{x})}{\rho^{2 / 3} k_{W}^{2} \Lambda_{0}^{j / 3}},
\end{aligned}
$$

where we have used (6.3) in the second line, and $\Lambda_{0}$ was defined above (1.2). (We refer to the explanations above (6.2) regarding the factor $k_{W}^{2}$.) The other terms appearing in (6.4) are bounded and differentiable functions of their arguments, except possibly $\tau_{j}$, but for this we have

$$
\left|\tau_{j+1}-\tilde{\tau}_{j+1}\right| \leq C\left(d\left(x_{j}, \tilde{x}_{j}\right)+d\left(x_{j+1}, \tilde{x}_{j+1}\right)\right) \leq C^{\prime} d\left(x_{j}, \tilde{x}_{j}\right)
$$

(see [CM, eq. (5.28)]). This combined with the previous estimate yields,

$$
\left|\ln J_{T^{-j} V_{0}}^{s} T^{-1}\left(x_{j}\right)-\ln J_{T^{-j} V^{s}}^{T^{-1}}\left(\tilde{x}_{j}\right)\right| \leq C\left(d(x, \tilde{x}) \Lambda_{0}^{-j / 3}\left(\rho^{-2 / 3} k_{W}^{-2}+1\right)+\phi_{j}+\phi_{j+1}\right),
$$

where $\phi_{j}$ is the angle formed by the tangent vectors to $T^{-j} V_{0}$ at $x_{j}$ and $T^{-j} V$ at $\tilde{x}_{j}$ (in particular $\phi_{0}=\phi(x, \tilde{x})$.

Now it follows from [CM, eq. (5.29)] that

$$
\phi_{j} \leq C\left(d(x, \tilde{x}) j \Lambda_{0}^{-j}+\phi_{0} \Lambda_{0}^{-j}\right),
$$

and summing over $j$ completes the required estimate on the difference of Jacobians.

Finally, we estimate $\ln J \mathbf{h}_{V^{n}}\left(x_{n}\right)$. Since the holonomy $\mathbf{h}_{V^{n}}: \bar{W}_{i} \rightarrow V^{n}$ corresponding to the seeding foliation is uniformly $\mathcal{C}^{2}$, its Jacobian is a $\mathcal{C}^{1}$ function of the distance and angle between the two curves. Thus using again [CM, eq. (5.29)],

$$
\left|\ln J \mathbf{h}_{V^{n}}\left(x_{n}\right)\right| \leq C\left(\phi\left(x_{n}, \tilde{x}_{n}\right)+d\left(x_{n}, \tilde{x}_{n}\right)\right) \leq C\left(d(x, \tilde{x}) n \Lambda_{0}^{-n}+\phi(x, \tilde{x}) \Lambda_{0}^{-n}\right) .
$$

Combining this with our previous estimate completes the proof of Lemma 6.4.

We adopt smooth local coordinates $\left(\bar{x}^{u}, \bar{x}^{s}\right) \in \mathcal{M}$ to write the foliation $\bar{\gamma}=\left(\bar{x}^{u}, \bar{G}\left(\bar{x}^{u}, \bar{x}^{s}\right)\right)$, where $\bar{x}^{u}$ and $\bar{x}^{s}$ range over the relevant intervals. The curve $V_{0}$ corresponds to $\left\{\left(0, \bar{x}^{s}\right): \bar{x}^{s} \in\right.$ $\left.\left[0,\left|V_{0}\right|\right]\right\}$, and (this is the first condition of (ii) for the map-foliation)

$$
\bar{G}\left(0, \bar{x}^{s}\right)=\bar{x}^{s} \text {. }
$$

This is the canonical straightening map of the foliation $\bar{G}$, with respect to the point $(0,0)$, i.e., the map sending the horizontal lines of $\mathbb{R}^{2}$ on the leaves of the foliation, preserving vertical lines and equal to the identity on $\{0\} \times \mathbb{R}$. In other words,

$$
\overline{\mathbb{F}}\left(\bar{x}^{u}, \bar{x}^{s}\right)=\left(\bar{x}^{u}, \bar{G}\left(\bar{x}^{u}, \bar{x}^{s}\right)\right)
$$

where $\bar{G}\left(\bar{x}^{u}, \bar{x}^{s}\right)$ is the first component of the point on the vertical through $\bar{x}^{u}$ on the leaf going through $\left(0, \bar{x}^{s}\right)$, with $\bar{G}\left(0, \bar{x}^{s}\right)=\bar{x}^{s}$. Geometrically, $\bar{x}^{s} \mapsto \bar{G}\left(\bar{x}^{u}, \bar{x}^{s}\right)$ is the holonomy $\mathbf{h}_{0 \rightarrow \bar{x}^{u}}$ of the foliation between the vertical transversals at 0 and $\bar{x}^{u}$, so that $\partial_{\bar{x}^{s}} \bar{G}\left(\bar{x}^{u}, \bar{x}^{s}\right)$ is the Jacobian 
of this holonomy between verticals. The image $U$ under $D \overline{\mathbb{F}}$ of the horizontal vector field is tangent to the foliation, and has first component equal to one, i.e., $U\left(\bar{x}^{u}, \bar{x}^{s}\right)=\left(1, u\left(\bar{x}^{u}, \bar{x}^{s}\right)\right)$, with $u\left(\overline{\mathbb{F}}\left(\bar{x}^{u}, \bar{x}^{s}\right)\right)=\partial_{\bar{x}^{u}} \bar{G}\left(\bar{x}^{u}, \bar{x}^{s}\right)$.

We next use Lemma 6.4 to derive the smoothness properties (v)-(vi) of this foliation. Write $V=\left\{\left(v\left(\bar{x}^{s}\right), \bar{x}^{s}\right)\right\}$ for some smooth function $v$. Taking $V=V_{c}$ in Lemma 6.4 to be the (purely vertical) curve defined by $v\left(\bar{x}^{s}\right) \equiv c$ for some constant $c$ (which we can do since the map-unstable cones are globally defined, i.e., they have a uniform width), we have by the above discussion,

$$
J \mathbf{h}_{V_{c}}\left(0, \bar{x}^{s}\right)=\partial_{\bar{x}^{s}} \bar{G}\left(c, \bar{x}^{s}\right) .
$$

Since $\tilde{x}=\mathbf{h}_{V}(x)$ implies $d(x, \tilde{x}) \leq C k_{W}^{2} \rho$, we have

$$
d(x, \tilde{x}) \rho^{-2 / 3} k_{W}^{-2} \leq C \rho^{1 / 3} .
$$

Thus Lemma 6.4 implies that $\partial_{\bar{x}^{s}} \bar{G}$ is bounded independently of $\rho$ (both above away from infinity and below away from 0$)$, proving the analogue of $(\mathrm{v})$ for $P^{+}(W)$.

The next lemma establishes the analogue of (vi) for $\bar{G}$.

Lemma 6.5. $\partial_{\bar{x}^{u}} \partial_{\bar{x}^{s}} \bar{G} \in \mathcal{C}^{0}$ and there exists $C>0$ such that $\left|\partial_{\bar{x}^{u}} \partial_{\bar{x}^{s}} \bar{G}\right|_{\infty} \leq C \rho^{-2 / 3} k_{W}^{-2}$.

Proof. Since we have chosen a smooth change of coordinates, angles in $(r, \varphi)$-coordinates are transformed smoothly to $\left(\bar{x}^{u}, \bar{x}^{s}\right)$-coordinates. Thus for the family of vertical stable curves $V_{c}$, we have $\phi_{V_{c}}(x, \tilde{x})$ varying as a smooth function of $d(x, \tilde{x})$.

We have

$$
\partial_{\bar{x}^{u}} \partial_{\bar{x}^{s}} \bar{G}\left(c, \bar{x}^{s}\right)=\partial_{c} J \mathbf{h}_{V_{c}}\left(0, \bar{x}^{s}\right) .
$$

First we prove the bound on $\left|\partial_{\bar{x}^{u}} \partial_{\bar{x}^{s}} \bar{G}\right|_{\infty}$. As before, let $V_{c}^{n}=T^{-n} V_{c}$, and let $\mathbf{h}_{V_{c}^{n}}$ denote the holonomy along $\{\bar{\gamma}\}$ from $V_{0}^{n}$ to $V_{c}^{n}$. Note that

$$
\frac{J \mathbf{h}_{V_{c+\delta}^{n}}}{J \mathbf{h}_{V_{c}^{n}}}=J \mathbf{h}_{V_{c}^{n}, V_{c+\delta}^{n}},
$$

where $\mathbf{h}_{V_{c}^{n}, V_{c+\delta}^{n}}$ is the holonomy map from $V_{c}^{n}$ to $V_{c+\delta}^{n}$. For each $\bar{x}^{s}$,

$$
\begin{aligned}
& \left|J \mathbf{h}_{V_{c+\delta}}\left(0, \bar{x}^{s}\right)-J \mathbf{h}_{V_{c}}\left(0, \bar{x}^{s}\right)\right|=\mid \frac{J_{V_{0}}^{s} T^{-n}\left(0, \bar{x}^{s}\right)}{J_{V_{c+\delta}}^{s} T^{-n}\left(\mathbf{h}_{V_{c+\delta}}\left(0, \bar{x}^{s}\right)\right)} J \mathbf{h}_{V_{c+\delta}^{n}}\left(T^{-n}\left(0, \bar{x}^{s}\right)\right) \\
& \quad-\frac{J_{V_{0}}^{s} T^{-n}\left(0, \bar{x}^{s}\right)}{J_{V_{c}}^{s} T^{-n}\left(\mathbf{h}_{V_{c}}\left(0, \bar{x}^{s}\right)\right)} J \mathbf{h}_{V_{c}^{n}\left(T^{-n}\left(0, \bar{x}^{s}\right)\right) \mid} \\
& =\frac{J_{V_{0}}^{s} T^{-n}\left(0, \bar{x}^{s}\right)}{J_{V_{c}}^{s} T^{-n}\left(\mathbf{h}_{V_{c}}\left(0, \bar{x}^{s}\right)\right)} J \mathbf{h}_{V_{c}^{n}}\left(T^{-n}\left(0, \bar{x}^{s}\right)\right) \\
& \quad \times\left|\frac{J_{V_{c}}^{s} T^{-n}\left(\mathbf{h}_{V_{c}}\left(0, \bar{x}^{s}\right)\right)}{J_{V_{c+\delta}}^{s} T^{-n}\left(\mathbf{h}_{V_{c+\delta}}\left(0, \bar{x}^{s}\right)\right)} J \mathbf{h}_{V_{c}^{n}, V_{c+\delta}^{n}}\left(T^{-n}\left(\mathbf{h}_{V_{c}}\left(0, \bar{x}^{s}\right)\right)\right)-1\right| \\
& \leq C \delta \rho^{-2 / 3} k_{W}^{-2},
\end{aligned}
$$

where in the last line, we have used the smooth dependence of $\phi_{V_{c}}(x, \bar{x})$ on $d(x, \bar{x})$ and replaced the factor $d(x, \bar{x})$ from Lemma 6.4 by $\delta$ since the estimates of that lemma hold for the holonomy between any two stable curves connected by the foliation $\{\bar{\gamma}\}$. (To bound the first factor via Lemma 6.4, we also used that $|c| \leq k_{W}^{2} \rho$ so that $1+c \rho^{-2 / 3} k_{W}^{-2} \leq C$.) Thus $\left|\partial_{\bar{x}^{u}} \partial_{\bar{x}^{s}} \bar{G}\right|_{\infty} \leq$ $C \rho^{-2 / 3} k_{W}^{-2}$, proving the required bound. 
To see that in fact $\partial_{\bar{x}^{u}} \partial_{\bar{x}^{s}} \bar{G}$ is continuous, write

$$
\partial_{\bar{x}^{u}} \partial_{\bar{x}^{s}} \bar{G}\left(c, \bar{x}^{s}\right)=\partial_{c} J \mathbf{h}_{V_{c}}\left(0, \bar{x}^{s}\right)=\partial_{c}\left(\frac{J_{V_{0}}^{s} T^{-n}\left(0, \bar{x}^{s}\right)}{J_{V_{c}}^{s} T^{-n}\left(\mathbf{h}_{V_{c}}\left(0, \bar{x}^{s}\right)\right)} J \mathbf{h}_{V_{c}^{n}}\left(T^{-n}\left(0, \bar{x}^{s}\right)\right)\right) .
$$

Since the seeding foliation is uniformly $\mathcal{C}^{2}$, the Jacobian $J \mathbf{h}_{V_{c}^{n}}$ is a $\mathcal{C}^{1}$ function of its base point and the angle $\phi\left(x_{n}, \tilde{x}_{n}^{c}\right)$ between the stable curves $V_{0}^{n}$ and $V_{c}^{n}$ at the points $x_{n}=T^{-n}\left(0, \bar{x}^{s}\right)$ and $\tilde{x}_{n}^{c}=T^{-n}\left(\mathbf{h}_{V_{c}}\left(0, \bar{x}^{s}\right)\right)$, respectively. Thus we need only consider the dependence of $\phi\left(x_{n}, \tilde{x}_{n}^{c}\right)$ on $c$.

Since we have adopted a smooth change of coordinates, the angle $\phi\left(x_{n}, \tilde{x}_{n}^{c}\right)$ is a smooth function of the difference in slopes $\mathcal{V}\left(x_{n}\right)-\mathcal{V}\left(\tilde{x}_{n}^{c}\right)$ of the stable curves $V_{0}^{n}$ and $V_{c}^{n}$ in $(r, \varphi)$ coordinates, at the points $x_{n}$ and $\tilde{x}_{n}^{c}$, respectively. By the time reversal of [CM, eq. (3.39)] we have $\mathcal{V}=\mathcal{B}^{-} \cos \varphi-\mathcal{K}$, where $\mathcal{B}^{-}$is the curvature of the wavefront just after impact (under the backwards flow). Thus,

$$
\partial_{c}\left(\mathcal{V}\left(x_{n}\right)-\mathcal{V}\left(\tilde{x}_{n}^{c}\right)\right)=-\partial_{c} \mathcal{K}\left(\tilde{x}_{n}^{c}\right)+\partial_{c} \cos \varphi\left(\tilde{x}_{n}^{c}\right) \mathcal{B}^{-}\left(\tilde{x}_{n}^{c}\right)+\cos \varphi\left(\tilde{x}_{n}^{c}\right) \partial_{c} \mathcal{B}^{-}\left(\tilde{x}_{n}^{c}\right) .
$$

Moreover, the curvature of the wave front $\mathcal{B}^{-}\left(\tilde{x}_{n}^{c}\right)$ can be expressed recursively as

$$
\mathcal{B}^{-}\left(\tilde{x}_{n}^{c}\right)=-\frac{2 \mathcal{K}\left(\tilde{x}_{n}^{c}\right)}{\cos \varphi\left(\tilde{x}_{n}^{c}\right)}+\frac{\mathcal{B}^{-}\left(\tilde{x}_{n-1}^{c}\right)}{1-\tau\left(\tilde{x}_{n}^{c}\right) \mathcal{B}^{-}\left(\tilde{x}_{n-1}^{c}\right)}
$$

where $\tilde{x}_{j}^{c}=T^{-j}\left(\mathbf{h}_{V_{c}}\left(0, \bar{x}^{s}\right)\right)$. Since our scatterers are $\mathcal{C}^{3}, \mathcal{K}$ is $\mathcal{C}^{1}$ so every term appearing above is a locally $\mathcal{C}^{1}$ function of its argument composed with $T^{-j}$ for some $j=1, \ldots, n$, except possibly $\mathcal{B}^{-}\left(\tilde{x}_{n-1}^{c}\right)$. Note that this is also true of $\tau$, even though the derivative of $\tau(x)$ has a term involving $\mathcal{K}(T x) / \cos (T x)$. To deal with $\mathcal{B}^{-}\left(\tilde{x}_{n-1}^{c}\right)$, simply apply relation (6.11) recursively with the chain rule to deduce that $\mathcal{B}^{-}\left(\tilde{x}_{n}^{c}\right)$ is continuously differentiable with respect to $c$ if $\mathcal{B}^{-}\left(\tilde{x}_{0}^{c}\right)$ is. But this last statement obviously holds since the curves $V_{c}$ are vertical segments in $\left(\bar{x}^{u}, \bar{x}^{s}\right)$ coordinates and the change of coordinates is smooth.

Differentiating with respect to $c$ in (6.10) and using the chain rule together with the above observations yields a continuous function (which we need not compute) times the unstable Jacobians $J_{\bar{\gamma}_{x_{0}}}^{u} T^{-j}\left(\mathbf{h}_{V_{c}}\left(x_{0}\right)\right)$, where $\bar{\gamma}_{x_{0}}$ is the unstable curve of the surviving foliation passing through $x_{0}$. This last factor is also continuous in $c$ since $T$ is locally $\mathcal{C}^{2}$.

Next we turn to the factor $J_{V_{c}}^{s} T^{-n}\left(\mathbf{h}_{V_{c}}\left(0, \bar{x}^{s}\right)\right)$ in (6.9). This is simply the product of factors of the form,

$$
J_{T^{-j} V_{c}}^{s} T^{-1}\left(\tilde{x}_{j}^{c}\right)=\frac{\cos \varphi\left(\tilde{x}_{j}^{c}\right)+\tau\left(\tilde{x}_{j}^{c}\right)\left(\mathcal{K}\left(\tilde{x}_{j}^{c}\right)-\mathcal{V}\left(\tilde{x}_{j}^{c}\right)\right)}{\cos \varphi\left(\tilde{x}_{j+1}^{c}\right)} \sqrt{\frac{1+\mathcal{V}\left(\tilde{x}_{j+1}^{c}\right)^{2}}{1+\mathcal{V}\left(\tilde{x}_{j}^{c}\right)^{2}}}, \quad j=0,1, \ldots n-1,
$$

using (6.4). All functions appearing here are again (locally) smooth functions of their arguments so that as above, differentiating with respect to $c$ and using (6.10) once more for $\partial_{c} \mathcal{V}\left(\tilde{x}_{j}^{c}\right)$, yields a sum of continuous functions times unstable Jacobians of the form $J_{\bar{\gamma}_{x_{0}}}^{u} T^{-j}\left(\mathbf{h}_{V_{c}}\left(x_{0}\right)\right)$, which are again continuous. This completes the verification of the continuity of $\partial_{\bar{x}^{u}} \partial_{\bar{x}^{s}} \bar{G}\left(c, \bar{x}^{s}\right)$ and thus the proof of Lemma 6.5.

Although the Jacobians of holonomy maps corresponding to billiards are not necessarily Hölder continuous (see [CM, p. 124]), we shall next see that the holonomy we have constructed here does have Hölder continuous Jacobian, leveraging the fact that the surviving foliation must have length $\rho$, as in the proof of Lemma 6.4.

Lemma 6.6 (Hölder continuity of the Jacobian of surviving foliation). Let $\{\bar{\gamma}\}$ be the surviving foliation transversal to $V_{0}$ introduced just before Lemma 6.4. Fix $C^{\prime}>0$ and consider the set of stable curves $V_{1}, V_{2} \in \mathcal{W}^{s}$ which are connected by the transverse foliation $\{\bar{\gamma}\}$ and such that the 
angles between $V_{1}$ and $V_{2}$ satisfy, $\phi\left(x, \mathbf{h}_{12}(x)\right) \leq C^{\prime} d\left(x, \mathbf{h}_{12}(x)\right)$ for all $x \in V_{1}$, where $\mathbf{h}_{12}$ is the holonomy map from $V_{1}$ to $V_{2}$. We require that $V_{0}$ be in this family. For any $0<\varpi \leq 1 / 15$, there exists $C>0$ such that given two such $V_{1}, V_{2} \in \mathcal{W}^{s}$, we have

$$
\begin{aligned}
& \left|\ln \frac{J \mathbf{h}_{12}\left(v_{1}\left(\bar{x}^{s}\right), \bar{x}^{s}\right)}{J \mathbf{h}_{12}\left(v_{1}\left(\bar{y}^{s}\right), \bar{y}^{s}\right)}\right| \\
& \leq C d\left(\bar{x}^{s}, \bar{y}^{s}\right)^{\varpi} d\left(V_{1}, V_{2}\right)^{1-\varpi} \max \left\{k_{W}^{-2(2+5 \varpi) / 3} \rho^{-2(1+\varpi) / 3}, k_{W}^{-82 \varpi / 3} \rho^{-38 \varpi / 3}\right\},
\end{aligned}
$$

where $V_{1}$ is the graph of the function $v_{1}$, and $\bar{x}^{s}, \bar{y}^{s}$ are in the domain $\left[0,\left|V_{0}\right|\right]$ of $v_{1}$.

The proof of the above lemma is in Appendix A, In view of (6.6), (6.8), and Remark 6.3, Lemma 6.6 gives the analogue of the four-point condition (vii) for the billiard map.

6.3. Interpolating the map-foliation across the gaps, (vi). We will fill in the gaps in the surviving foliation by interpolation, to obtain a full foliation in a $k_{W}^{2} \rho$ neighborhood of $P^{+}(W)$. More precisely, we will first obtain bounds on the Lipschitz norm of the tangent vector $\partial_{u} \bar{F}$ to the foliation across the gaps. Interpolating linearly between the Jacobians, following $\mathrm{BL}$, App. D] (see (6.3) ) these bounds will then give the analogue of (vi) for the map, while the analogue of (vii) for the map will be discussed in Section 6.4.

First, if there are gaps at the endpoints of $P^{+}(W)$, we may trivially extend the surviving foliation smoothly across these gaps since no interpolation is necessary.

Gaps are created by the intersection of $T^{n-j}(\ell)$ with a singularity curve or 30 with boundaries of homogeneity strips, since to not be cut under the original scheme means to lie in a single homogeneity strip. Some gaps are created by an intersection with the boundary of a single homogeneity strip. If we choose not to cut those curves $T^{n-j}(\ell)$ which cross these two homogeneity strips, we will just be considering unstable curves that lie in two adjacent strips. The expansion and contraction factors along these curves still enjoy bounded distortion, with a slightly larger distortion constant. We fill these gaps by not cutting the transverse foliation at time $n-j$.

The parts of the foliation that cross more than two strips will belong to the gap near $\mathcal{S}_{0}$ for which all curves are cut. For that gap, we linearly interpolate between the surviving curves at each end of the gap at time 0 , and for this we shall estimate the Lipschitz constant of the interpolation.

If $T^{-j}\left(P^{+}(W)\right)$ lands in a neighborhood of $\mathcal{S}_{0}$ it may cross countably many homogeneity strips. Since we enforce a definite length scale for the transverse foliation, this means that the foliation is cut in all homogeneity strips above a certain index (depending on the length of $T^{n-j}(\ell)$ ). So we can consider this to be a single gap in our construction (even though $T^{-j}\left(P^{+}(W)\right.$ ) is subdivided into countably many homogeneous curves).

With this construction, since $\varkappa>0$ is fixed, there are finitely many gaps in the foliation of $P^{+}(W)$, each gap containing one or more intersections of $P^{+}(W)$ with a curve in $\mathcal{S}_{-n}, n \leq$ $\left\lfloor\frac{\varkappa}{\tau_{\min }}\right\rfloor+1$.

Now fix one gap and choose a segment $V_{0}$ of $P^{+}(W)$ with surviving foliation on one side of the gap. This fixes $n=n\left(V_{0}\right)$ as the number of iterates appearing in the definition of the foliation on $V_{0}$. Let $j$ be the least integer $j^{\prime} \geq 1$ such that an element of $T^{j^{\prime}}\left(\mathcal{S}_{0}\right)$ intersects $P^{+}(W)$ in the gap. (Note that $j \leq n$ must exist otherwise there would be no gap.) Thus $V_{0}$ is contained in a longer curve $V_{1} \subset P^{+}(W)$ such that $T^{-m}\left(V_{1}\right)$ is a homogeneous stable curve for $m=0,1, \ldots, j-1$ and which is cut for the first time at time $-j$. We prove (vi) for the interpolated foliation by considering two cases.

${ }^{30}$ We use implicitly here that $T^{-j}\left(P^{+}(W)\right)$ crosses an extended singularity line only if there exists $\ell$ as constructed in Step 1 so that $T^{n-j}(\ell)$ crosses a singularity line. 
Case 1. $T^{-j}\left(V_{1}\right) \cap \mathcal{S}_{0} \neq \emptyset$. This implies that $T^{-j}\left(V_{1}\right)$ crosses countably many homogeneity strips $\left(T^{-j}\left(V_{1}\right)\right.$ is not assumed to be homogeneous). At this step, each $\bar{\gamma}$ intersecting $V_{0}$ is such that $T^{-j}(\bar{\gamma})$ is a homogeneous unstable curve of length $k_{W}^{2} \rho / J_{T^{-j}(\bar{\gamma})}^{u} T^{j}(z)$, for some $z \in T^{-j}(\bar{\gamma})$. This implies that the index $k_{j}$ of the homogeneity strip containing this curve satisfies

$$
k_{j}^{-3}=C^{ \pm 1} \frac{k_{W}^{2} \rho}{J_{T^{-j}(\bar{\gamma})}^{u} T^{j}(z)},
$$

for some uniform constant $C$ and any $z \in T^{-j}(\bar{\gamma})$. (Compare with (6.3).) Thus the length of the image of the gap in $T^{-j}\left(V_{1}\right)$ is of order $k_{j}^{-2}$. Since this is the first time $V_{1}$ is being cut, $T^{-j+1}\left(V_{1}\right)$ is still a homogeneous stable curve so, using the fact that the expansion from $T^{-j+1}\left(V_{1}\right)$ to $T^{-j}\left(V_{1}\right)$ in the gap is of order $k_{j}^{2}$, we conclude that for any $x \in V_{1}$ the length of the gap in $V_{1}$ is

$$
\left|V_{1}^{\text {gap }}\right|=C^{ \pm 1} k_{j}^{-4} / J_{V_{1}}^{s} T^{-j+1}(x) .
$$

In order to estimate the Lipschitz constant of the foliation with which we want to fill in the gap, we must calculate the largest possible angle between the edge of the surviving foliation in $V_{0}$ and the part of $T^{j}\left(\mathcal{S}_{0}\right)$ crossing $P^{+}(W)$. Note that $T^{j}\left(\mathcal{S}_{0}\right)$ is necessarily an unstable curve, and indeed, the tangent vector to $\mathcal{S}_{0}$ is mapped into the unstable cone in one step.

Using [CM, eq. (3.39)], we see that the image under DT of an unstable curve has slope $\mathcal{V}_{1}$ (in $(r, \varphi)$ coordinates) given by

$$
\mathcal{V}_{1}=\frac{\cos \varphi_{1}}{\tau_{0}+\frac{\cos \varphi_{0}}{\mathcal{V}_{0}+\mathcal{K}_{0}}}+\mathcal{K}_{1}
$$

where objects with subscript 0 correspond to a base point $x$ and objects with subscript 1 correspond to $T(x)$.

Let $x=\mathcal{S}_{0} \cap T^{-j} V_{1}$. Now since the slope $\mathcal{V}_{0}(x)=0$ and $\varphi_{0}(x)=\pi / 2$, the slope of the image of this vector after one step is given by

$$
\mathcal{V}_{1}(x)=\mathcal{K}_{1}+\frac{\cos \varphi_{1}}{\tau_{0}}
$$

Let $y$ denote the endpoint of $T^{-j}\left(V_{0}\right)$ adjacent to the gap and let $\bar{\gamma}$ denote the unstable curve in the constructed foliation containing $T^{j}(y)$. Recall that $\bar{\gamma}=T^{n}(\ell)$ for some curve $\ell$ of the unstable seeding foliation of a neighborhood of a curve $W_{i} \in \mathcal{G}_{\varkappa}(W)$; thus we do not make any assumption on the slope of $T^{-j} \bar{\gamma}=T^{n-j}(\ell)$, other than that it lies in the unstable cone at $y$.

Letting $\phi^{\bar{\gamma}}(T(x), T(y))$ denote the angle between $T\left(\mathcal{S}_{0}\right)$ and $T^{-j+1}(\bar{\gamma})$ at $T(x)$ and $T(y)$ respectively, we estimate,

$$
\begin{aligned}
\phi^{\bar{\gamma}}(T x, T y) \leq & \left|\mathcal{V}_{1}(x)-\mathcal{V}_{1}(y)\right| \leq\left|\mathcal{K}_{1}(x)-\mathcal{K}_{1}(y)\right|+\left|\frac{\cos \varphi_{1}(x)}{\tau_{0}(x)}-\frac{\cos \varphi_{1}(y)}{\tau_{0}(y)+\frac{\cos \varphi_{0}(y)}{\mathcal{V}_{0}(y)+\mathcal{K}_{0}(y)}}\right| \\
\leq & |\mathcal{K}(T x)-\mathcal{K}(T y)|+\frac{1}{\tau_{\min }}|\cos \varphi(T x)-\cos \varphi(T y)| \\
& +\cos \varphi(T y)\left|\frac{1}{\tau(x)}-\frac{1}{\tau(y)}\right|+\cos \varphi(T y)\left|\frac{1}{\tau(y)}-\frac{1}{\tau(y)+\frac{\cos \varphi_{0}(y)}{\mathcal{V}_{0}(y)+\mathcal{K}_{0}(y)}}\right| .
\end{aligned}
$$

The first two differences above are bounded by a uniform constant times $d(T(x), T(y))$, which is of order $k_{j}^{-4}$. As before, $|\tau(x)-\tau(y)| \leq C(d(x, y)+d(T(x), T(y))) \leq C^{\prime} d(x, y) \leq C^{\prime} k_{j}^{-2}$. Finally, the last difference is of order $\cos \varphi(y)=C k_{j}^{-2}$. Putting these estimates together, we obtain

$$
\phi^{\bar{\gamma}}(T(x), T(y)) \leq C k_{j}^{-4}+C k_{j}^{-2} \cos \varphi(T y) .
$$


The quantity to be estimated is $\frac{\phi^{\bar{\gamma}}\left(T^{j}(x), T^{j}(y)\right)}{d\left(T^{j}(x), T^{j}(y)\right)}$. This will yield the Lipschitz constant of our interpolated foliation in the gap. To do this, we note that [CM, eq. (5.27) and following p. 122] imply

$$
\begin{aligned}
\phi^{\bar{\gamma}}\left(T^{j}(x), T^{j}(y)\right)=Q_{j}+ & \cos \varphi\left(T^{j}(y)\right) Q_{j-1} \\
& +\frac{\cos \varphi\left(T^{j}(y)\right)}{\cos \varphi\left(T^{j-1}(y)\right)} \frac{\phi^{\bar{\gamma}}\left(T^{j-1}(x), T^{j-1}(y)\right)}{\left(1+\tau\left(T^{j-1}(y)\right) B^{+}\left(T^{j-1} y\right)\right)\left(1+\tau\left(T^{j-1} x\right) B^{+}\left(T^{j-1}(x)\right)\right)},
\end{aligned}
$$

where $B^{+}$denotes the post-collisional curvature of the unstable wavefront and the functions $Q_{j}, Q_{j-1}$ satisfy $\left|Q_{j}\right| \leq C d\left(T^{j}(x), T^{j}(y)\right)$ and $\left|Q_{j-1}\right| \leq C d\left(T^{j-1}(x), T^{j-1}(y)\right)$. Proceeding inductively and recalling the expressions for the stable and unstable Jacobians given by (6.4) and (A.4), we obtain the following expression for the difference in angles,

$$
\begin{aligned}
& \phi^{\bar{\gamma}}\left(T^{j} x, T^{j} y\right) \leq C \sum_{m=0}^{j-2} \frac{d\left(T^{j-m} x, T^{j-m} y\right)+\cos \varphi\left(T^{j-m} y\right) d\left(T^{j-m-1} x, T^{j-m-1} y\right)}{J_{T^{m-j}}^{u} T_{\bar{\gamma}}^{T^{m}}\left(T^{j-m} y\right) J_{V_{0}}^{s} T^{-m}\left(T^{j} y\right)} \\
& +\frac{\phi^{\bar{\gamma}}(T x, T y)}{J_{T^{1-j}}^{u} T^{T^{j-1}}(T y) J_{V_{0}}^{s} T^{-j+1}\left(T^{j} y\right)} .
\end{aligned}
$$

For each $m$ in the sum we have the following two bounds: For the first term in the numerator, $d\left(T^{j-m}(x), T^{j-m}(y)\right) / J_{V_{0}}^{s} T^{-m}\left(T^{j} y\right) \leq C d\left(T^{j}(x), T^{j}(y)\right)$. For the second term in the numerator, there is a gap in the expansion factors, so we need to add the stable Jacobian, $J_{T^{-m} V_{0}}^{s} T^{-1}\left(T^{j-m}(y)\right)$ as follows:

$$
\frac{\cos \varphi\left(T^{j-m} y\right) d\left(T^{j-m-1} x, T^{j-m-1} y\right)}{J_{V_{0}}^{s} T^{-m}\left(T^{j} y\right)} \leq \cos \varphi\left(T^{j-m} y\right) d\left(T^{j} x, T^{j} y\right) J_{T^{-m} V_{0}}^{s} T^{-1}\left(T^{j-m} y\right) .
$$

Now since $T^{j-m-1}(y) \in \mathbb{H}_{k_{m+1}}$, we have $J_{T^{-m} V_{0}}^{s} T^{-1}\left(T^{j-m} y\right) \leq C k_{m+1}^{2}$. Also, in preparation for using (6.12), we note that

$$
\begin{aligned}
J_{T^{-m-1} \bar{\gamma}}^{u} T^{m+1}\left(T^{j-m-1}(y)\right) & =J_{T^{-m} \bar{\gamma}}^{u} T^{m}\left(T^{j-m} y\right) J_{T^{-m-1} \bar{\gamma}}^{u} T\left(T^{j-m-1}(y)\right) \\
& \leq C J_{T^{-m} \bar{\gamma}}^{u} T^{m}\left(T^{j-m} y\right) k_{m}^{2} .
\end{aligned}
$$

Putting these facts together and using (6.12), we estimate

$$
\begin{aligned}
& \frac{\cos \varphi\left(T^{j-m}(y)\right) d\left(T^{j-m-1}(x), T^{j-m-1}(y)\right)}{J_{T^{m-j}}^{u} T^{m}\left(T^{j-m}(y)\right) J_{V_{0}}^{s} T^{-m}\left(T^{j}(y)\right)} \leq \frac{\cos \varphi\left(T^{j-m} y\right) d\left(T^{j} x, T^{j} y\right) J_{T^{-m} V_{0}}^{s} T^{-1}\left(T^{j-m} y\right)}{J_{T^{m-j}}^{u} T^{m}\left(T^{j-m} y\right)} \\
& \quad \leq C \frac{k_{m}^{-2} d\left(T^{j} x, T^{j} y\right)}{J_{T^{m-j}}^{u} T^{m}\left(T^{j-m} y\right)} k_{m+1}^{2} \leq C \frac{k_{m}^{-2} d\left(T^{j}(x), T^{j}(y)\right)}{J_{T^{m-j} \bar{\gamma}}^{u} T^{m}\left(T^{j-m} y\right)} \frac{\left(J_{T^{m-j}}^{u} T^{m}\left(T^{j-m} y\right)\right)^{2 / 3} k_{m}^{4 / 3}}{k_{W}^{4 / 3} \rho^{2 / 3}} \\
& \leq C d\left(T^{j} x, T^{j} y\right) \Lambda_{0}^{-m / 3} k_{W}^{-4 / 3} \rho^{-2 / 3} .
\end{aligned}
$$

Collecting this bound together with (6.13), (6.14), and (6.15), we obtain (note that $\left|V_{1}^{\text {gap }}\right|=$ $\left.d\left(T^{j}(x), T^{j}(y)\right)\right)$

$$
\begin{aligned}
\frac{\phi^{\bar{\gamma}}\left(T^{j}(x), T^{j}(y)\right)}{d\left(T^{j}(x), T^{j}(y)\right)} & \leq C \sum_{m=0}^{j-2} \frac{k_{W}^{-4 / 3}}{\Lambda_{0}^{m / 3} \rho^{2 / 3}}+C \frac{k_{j}^{-4}+\cos \varphi(T y) k_{j}^{-2}}{J_{T^{1-j}}^{u} T^{j-1}(T y) J_{V_{0}}^{s} T^{-j+1}\left(T^{j} y\right)} \cdot \frac{J_{V_{0}}^{s} T^{-j+1}\left(T^{j} y\right)}{k_{j}^{-4}} \\
& \leq C k_{W}^{-4 / 3} \rho^{-2 / 3}+\frac{C}{J_{T^{1-j} \bar{\gamma}}^{u} T^{j-1}(T y)}+\frac{k_{j}^{2} \cos \varphi(T y)}{J_{T^{1-j} \bar{\gamma}^{j-1}(T y)}^{T^{j}}} .
\end{aligned}
$$


Now using (6.12), we bound the last term by

$$
\frac{k_{j}^{2} \cos \varphi(T y)}{J_{T^{1-j} \bar{\gamma}}^{u} T^{j-1}(T y)} \leq \frac{\cos \varphi(T y)\left(J_{T^{-j} \bar{\gamma}}^{u} T(y)\right)^{2 / 3}}{\left(J_{T^{1-j}}^{u} T^{j-1}(T y)\right)^{1 / 3} k_{W}^{4 / 3} \rho^{2 / 3}} .
$$

Finally using the fact that $\left.J_{T^{-j} \bar{\gamma}}^{u} T(y)\right) \leq C / \cos \varphi(T y)$, we conclude

$$
\frac{\phi^{\bar{\gamma}}\left(T^{j} x, T^{j} y\right)}{d\left(T^{j} x, T^{j} y\right)} \leq C+C k_{W}^{-4 / 3} \rho^{-2 / 3} .
$$

This ends the proof of (vi) for the interpolation in Case 1.

Case 2. $T^{-j}\left(V_{1}\right) \cap \mathcal{S}_{0}=\emptyset$. This implies that $T^{-j+1}\left(V_{1}\right)$ lies on one side of a singularity curve in $\mathcal{S}_{-1}$, and that one endpoint of $T^{-j}\left(V_{1}\right)$ lies on a singularity curve in $\mathcal{S}_{1}$, but not on $\mathcal{S}_{0}$. If $V_{1}$ is such a curve containing a gap, then the other side of the gap necessarily contains a curve satisfying the condition of Case 1 above. Since the estimate of the angle difference carried out there was a worst-case estimate (in fact, we started with an angle outside the unstable cone, while in Case 2, the angle of the singularity curve in $\mathcal{S}_{-1}$ will necessarily be inside the unstable cone), the interpolated foliation across the entire gap can only have a better Lipschitz constant since we are increasing the distance we have to carry out the interpolation while not making the difference in angles any larger.

Other curves in $\mathcal{S}_{-n}$ may also cross the same gap. But these additional cuts can only make the gap longer, while again making the difference in angles no worse. Thus they do not make the Lipschitz constant any larger than the bound derived in Case 1.

We next show that the Lipschitz control (6.16) that we obtained gives the $L^{\infty}$ bound (vi) on the foliation, essentially following the construction from Appendix D of [BL] (the main difference is that we shall not use induction).

Let $\left(0, \bar{x}^{s}\right), \bar{x}^{s} \in\left[a^{\prime}, b^{\prime}\right]$, denote the stable curve $P^{+}(W)$ and the interval $[a, b], a^{\prime}<a<b<b^{\prime}$, denote the interval on which the foliation must be interpolated. The surviving foliation on both sides of the gap is given by $\bar{\gamma}_{a}\left(\bar{x}^{u}, \bar{x}^{s}\right)=\left(\bar{x}^{u}, \bar{G}_{a}\left(\bar{x}^{u}, \bar{x}^{s}\right)\right)$ and $\bar{\gamma}_{b}\left(\bar{x}^{u}, \bar{x}^{s}\right)=\left(\bar{x}^{u}, \bar{G}_{b}\left(\bar{x}^{u}, \bar{x}^{s}\right)\right.$. Next, fix $\bar{\varphi} \in \mathcal{C}^{2}([0,1],[0,1])$ satisfying $\bar{\varphi}(0)=\bar{\varphi}^{\prime}(0)=\bar{\varphi}^{\prime}(1)=0$ and $\bar{\varphi}(1)=1$. Define $\bar{\psi}=\left(1-c_{1}\right) \bar{\varphi}^{\prime}$, where $c_{1}$ will be chosen later small enough to ensure the interpolated foliation comprises unstable curves. Then $\bar{\psi}(0)=\bar{\psi}(1)=0$ and $\int_{0}^{1} \bar{\psi}=1-c_{1}$.

Define $\varphi\left(\bar{x}^{s}\right)=\bar{\varphi}\left(\frac{\bar{x}^{s}-a}{b-a}\right)$ and $\psi\left(\bar{x}^{s}\right)=\bar{\psi}\left(\frac{\bar{x}^{s}-a}{b-a}\right)$. It follows that $\varphi(a)=\partial_{\bar{x}^{s}} \varphi(a)=\partial_{\bar{x}^{s}} \varphi(b)=$ $\psi(a)=\psi(b)=0, \varphi(b)=1, \int_{a}^{b} \psi=\left(1-c_{1}\right)(b-a)$. Now define for all $\bar{x}^{s} \in[a, b]$ and $\left|\bar{x}^{u}\right| \leq k_{W}^{2} \rho$,

$$
\begin{aligned}
\theta_{0}\left(\bar{x}^{u}, \bar{x}^{s}\right) & =\left(\partial_{\bar{x}^{s}} \bar{G}_{b}\left(\bar{x}^{u}, b\right) \frac{\bar{x}^{s}-a}{b-a}+\partial_{\bar{x}^{s}} \bar{G}_{a}\left(\bar{x}^{u}, a\right) \frac{b-\bar{x}^{s}}{b-a}\right)\left(1-\psi\left(\bar{x}^{s}\right)\right) \\
\theta\left(\bar{x}^{u}, \bar{x}^{s}\right) & =\theta_{0}\left(\bar{x}^{u}, \bar{x}^{s}\right)-\frac{\psi\left(\bar{x}^{s}\right)}{\left(1-c_{1}\right)(b-a)} \int_{a}^{b} \theta_{0}\left(\bar{x}^{u}, z\right) d z \\
\sigma\left(\bar{x}^{u}, \bar{x}^{s}\right) & =\bar{G}_{b}\left(\bar{x}^{u}, b\right) \varphi\left(\bar{x}^{s}\right)+\bar{G}_{a}\left(\bar{x}^{u}, a\right)\left(1-\varphi\left(\bar{x}^{s}\right)\right)+\int_{a}^{\bar{x}^{s}} \theta\left(\bar{x}^{u}, z\right) d z .
\end{aligned}
$$

The foliation in the gap is then defined by $\bar{\gamma}\left(\bar{x}^{u}, \bar{x}^{s}\right)=\left(\bar{x}^{u}, \sigma\left(\bar{x}^{u}, \bar{x}^{s}\right)\right)$. With these definitions, $\sigma\left(\bar{x}^{u}, a\right)=\bar{G}_{a}\left(\bar{x}^{u}, a\right)$ and $\sigma\left(\bar{x}^{u}, b\right)=\bar{G}_{b}\left(\bar{x}^{u}, b\right)$. As shown in [BL, Appendix D], $\bar{\gamma} \in \mathcal{C}^{1+\text { Lip }}$, and in particular, $C^{-1} \leq\left|\partial_{\bar{x}^{s}} \sigma\right| \leq C$, proving the analogue of (v) for the interpolated foliation; moreover, choosing $c_{1}$ small enough guarantees that the curves of the interpolated foliation are unstable curves.

To prove (vi), note that

$$
\partial_{\bar{x}^{u}} \partial_{\bar{x}^{s}} \sigma\left(\bar{x}^{u}, \bar{x}^{s}\right)=\left(\partial_{\bar{x}^{u}} \bar{G}_{b}\left(\bar{x}^{u}, b\right)-\partial_{\bar{x}^{u}} \bar{G}_{a}\left(\bar{x}^{u}, a\right)\right) \varphi^{\prime}\left(\bar{x}^{s}\right)+\partial_{\bar{x}^{u}} \theta\left(\bar{x}^{u}, \bar{x}^{s}\right) .
$$


Now, the second term on the right-hand side is bounded by the supremum of the surviving foliations $\partial_{\bar{x}^{u}} \partial_{\bar{x}^{s}} \bar{G}_{a}$ and $\partial_{\bar{x}^{u}} \partial_{\bar{x}^{s}} \bar{G}_{b}$ by a straightforward calculation of $\partial_{\bar{x}^{u}} \theta$, and this is bounded by $C k_{W}^{-2} \rho^{-2 / 3}$, by Lemma 6.5 .

To bound the first term on the right-hand side, we use our Lipschitz bound (6.16). Note that $\bar{G}_{a}(\eta, a)$ parametrizes the curve on one side of the gap, while $\bar{G}_{b}(\eta, b)$ parametrizes the curve on the other. Equation (6.16) says

$$
\frac{\phi\left(\bar{G}_{a}\left(\bar{x}^{u}, a\right), \bar{G}_{b}\left(\bar{x}^{u}, b\right)\right)}{d\left(\bar{G}_{a}\left(\bar{x}^{u}, a\right), \bar{G}_{b}\left(\bar{x}^{u}, b\right)\right)} \leq C k_{W}^{-4 / 3} \rho^{-2 / 3},
$$

where $\phi$ represents the angle between the two unstable curves at the given points and the distance between points is measured along a stable curve connecting the two points. Since stable curves have uniformly bounded curvature, this distance is uniformly equivalent to Euclidean distance on the scatterer.

Since $\partial_{\bar{x}^{u}} \bar{G}$ represents the slope of an unstable curve, we have

$$
\begin{aligned}
& \phi\left(\bar{G}_{a}\left(\bar{x}^{u}, a\right), \bar{G}_{b}\left(\bar{x}^{u}, b\right)\right)=\left|\tan ^{-1}\left(\partial_{\bar{x}^{u}} \bar{G}_{a}\left(\bar{x}^{u}, a\right)\right)-\tan ^{-1}\left(\partial_{\eta} \bar{G}_{b}\left(\bar{x}^{u}, b\right)\right)\right| \\
& \quad=\frac{1}{1+z^{2}}\left|\partial_{\bar{x}^{u}} \bar{G}_{a}\left(\bar{x}^{u}, a\right)-\partial_{\bar{x}^{u}} \bar{G}_{b}\left(\bar{x}^{u}, b\right)\right| \geq \frac{1}{1+K_{0}^{2}}\left|\partial_{\bar{x}^{u}} \bar{G}_{a}\left(\bar{x}^{u}, a\right)-\partial_{\bar{x}^{u}} \bar{G}_{b}\left(\bar{x}^{u}, b\right)\right|
\end{aligned}
$$

for some $z \in \mathbb{R}$, and where $K_{0}$ denotes the maximum slope in the unstable cone (recall that we have global stable and unstable cones for the map). This estimate together with (6.16) yields,

$$
\left|\partial_{\bar{x}^{u}} \bar{G}_{a}\left(\bar{x}^{u}, a\right)-\partial_{\bar{x}^{u}} \bar{G}_{b}\left(\bar{x}^{u}, b\right)\right| \leq C K_{0}^{2} k_{W}^{-4 / 3} \rho^{-2 / 3} d\left(\bar{G}_{a}\left(\bar{x}^{u}, a\right), \bar{G}_{b}\left(\bar{x}^{u}, b\right)\right) .
$$

Now using this together with the fact that $\left|\varphi^{\prime}\right| \leq C\left(d\left(\bar{G}_{a}\left(\bar{x}^{u}, a\right), \bar{G}_{b}\left(\bar{x}^{u}, b\right)\right)\right)^{-1}$ due to the rescaling, we estimate the first term on the right-hand side of (6.17) by $C k_{W}^{-4 / 3} \rho^{-2 / 3}$. This completes the estimate we need for the $L^{\infty}$ norm (vi) for the interpolated map-foliation. Continuity of $\partial_{\bar{x}^{u}} \partial_{\bar{x}^{s}} \sigma$ follows immediately from (6.17) since all functions appearing on the right-hand side are continuous (using the continuity of $\partial_{\bar{x}^{u}} \partial_{\bar{x}^{s}} \bar{G}$ in the surviving foliation by Lemma 6.5). The continuity extends to the boundary of the gap since $\partial_{\bar{x}^{u}} \partial_{\bar{x}^{s}} \sigma\left(\bar{x}^{u}, a\right)=\partial_{\bar{x}^{u}} \theta_{0}\left(\bar{x}^{u}, a\right)=\partial_{\bar{x}^{u}} \partial_{\bar{x}^{s}} \bar{G}_{a}\left(\bar{x}^{u}, a\right)$, and similarly for $\partial_{\bar{x}^{u}} \partial_{\bar{x}^{s}} \sigma\left(\bar{x}^{u}, b\right)$.

6.4. Checking (vii) for the map-foliation interpolated across the gaps. In this step, we prove the analogue of Lemma 6.6 across gaps and in the part of the foliation that has been filled by interpolation, this will give the four-point estimate (vii) across the gaps. We begin by proving a lemma which will allow us to control the Hölder continuity of $\partial_{\bar{x}^{s}} \sigma$ defined by interpolation in each gap, specifically that the average slope of the interpolated foliation across the gap and the derivatives $\partial_{\bar{x}^{s}} \bar{G}$ on either side of the gap are close in the length scale of the gap.

Lemma 6.7. Let $P^{+}(W)$ be a stable curve parametrized by $\bar{x}^{s} \in\left[a^{\prime}, b^{\prime}\right]$ as in Subsection 6.3 and suppose the interval $(a, b) \subset\left[a^{\prime}, b^{\prime}\right]$ is a gap in the surviving foliation pushed forward $n$ steps. Then there exists $C>0$ such that for any $\bar{x}^{u}$ with $\left|\bar{x}^{u}\right| \leq k_{W}^{2} \rho$,

$$
\left|\partial_{\bar{x}^{s}} \bar{G}_{a}\left(\bar{x}^{u}, a\right)-\frac{\bar{G}_{b}\left(\bar{x}^{u}, b\right)-\bar{G}_{a}\left(\bar{x}^{u}, a\right)}{b-a}\right| \leq C \rho^{-31 / 105}|a-b|^{1 / 7} .
$$

A similar bound holds for $\partial_{\bar{x}^{s}} \bar{G}_{b}\left(\bar{x}^{u}, b\right)$.

Lemma 6.7 is proved in Appendix A. It allows us to prove the four-point estimate on the interpolated foliation in the gap. Let $a \leq \bar{x}^{s}<\bar{y}^{s} \leq b$, and $-k_{W}^{2} \rho \leq \bar{x}^{u}<\bar{y}^{u} \leq k_{W}^{2} \rho$ be the 
coordinates of arbitrary points in the gap. For our first estimate, the estimate on $\partial_{\bar{x}^{u}} \partial_{\bar{x}^{s}} \sigma$ from Subsection 6.3 allows us to write,

$$
\begin{aligned}
& \left|\partial_{\bar{x}^{s}} \sigma\left(\bar{x}^{u}, \bar{x}^{s}\right)-\partial_{\bar{x}^{s}} \sigma\left(\bar{x}^{u}, \bar{y}^{s}\right)-\partial_{\bar{x}^{s}} \sigma\left(\bar{y}^{u}, \bar{x}^{s}\right)+\partial_{\bar{x}^{s}} \sigma\left(\bar{y}^{u}, \bar{y}^{s}\right)\right| \\
& =\left|\int_{\bar{x}^{u}}^{\bar{y}^{u}} \partial_{\bar{x}^{u}} \partial_{\bar{x}^{s}} \sigma\left(z, \bar{y}^{s}\right)-\partial_{\bar{x}^{u}} \partial_{\bar{x}^{s}} \sigma\left(z, \bar{x}^{u}\right) d z\right| \leq C k_{W}^{-4 / 3} \rho^{-2 / 3}\left|\bar{x}^{u}-\bar{y}^{u}\right| .
\end{aligned}
$$

We will use Lemma 6.7 to produce a second estimate on the four-point difference above. In the following calculation, for brevity, we set $M_{a}=\bar{G}_{a}\left(\bar{x}^{u}, a\right), M_{b}=\bar{G}_{b}\left(\bar{x}^{u}, b\right), M_{a}^{\prime}=\partial_{\bar{x}^{s}} \bar{G}_{a}\left(\bar{x}^{u}, a\right)$ and $M_{b}^{\prime}=\partial_{\bar{x}^{s}} \bar{G}_{b}\left(\bar{x}^{u}, b\right)$. According to the definition of $\sigma$, we have for $\bar{x}^{s} \in[a, b]$,

$$
\partial_{\bar{x}^{s}} \partial_{\bar{x}^{s}} \sigma\left(\bar{x}^{u}, \bar{x}^{s}\right)=\left(M_{b}-M_{a}\right) \varphi^{\prime \prime}\left(\bar{x}^{s}\right)+\partial_{\bar{x}^{s}} \theta_{0}\left(\bar{x}^{u}, \bar{x}^{s}\right)-\frac{\psi^{\prime}\left(\bar{x}^{s}\right)}{\left(1-c_{1}\right)(b-a)} \int_{a}^{b} \theta_{0}\left(\bar{x}^{u}, z\right) d z .
$$

In addition, using the definition of $\theta_{0}$,

$$
\begin{aligned}
\partial_{\bar{x}^{s}} \theta_{0}\left(\bar{x}^{u}, \bar{x}^{s}\right) & =\frac{M_{b}^{\prime}-M_{a}^{\prime}}{b-a}\left(1-\psi\left(\bar{x}^{s}\right)\right)-\left(M_{b}^{\prime} \frac{\bar{x}^{s}-a}{b-a}+M_{a}^{\prime} \frac{b-\bar{x}^{s}}{b-a}\right) \psi^{\prime}\left(\bar{x}^{s}\right) \quad \text { and } \\
\int_{a}^{b} \theta_{0}\left(\bar{x}^{u}, z\right) d z & =\int_{a}^{b} M_{b}^{\prime} \frac{z-a}{b-a}+M_{a}^{\prime} \frac{b-z}{b-a} d z-\int_{a}^{b}\left(M_{b}^{\prime} \frac{z-a}{b-a}+M_{a}^{\prime} \frac{b-z}{b-a}\right) \psi(z) d z \\
& =\left(M_{b}^{\prime}+M_{a}^{\prime}\right) \frac{b-a}{2}-\int_{a}^{b}\left(M_{b}^{\prime} \frac{z-a}{b-a}+M_{a}^{\prime} \frac{b-z}{b-a}\right) \psi(z) d z
\end{aligned}
$$

Now combining these expressions, and recalling that by construction $\psi^{\prime}=(b-a)^{-1} \bar{\psi}^{\prime}$ and $\varphi^{\prime \prime}=\bar{\varphi}^{\prime \prime}(b-a)^{-2}=\frac{\bar{\psi}^{\prime}}{\left(1-c_{1}\right)(b-a)^{2}}$, we have

$$
\begin{aligned}
\partial_{\bar{x}^{s}} \partial_{\bar{x}^{s}} \sigma\left(\bar{x}^{u}, \bar{x}^{s}\right)= & \left(M_{b}-M_{a}\right) \varphi^{\prime \prime}\left(\bar{x}^{s}\right)+\frac{M_{b}^{\prime}-M_{a}^{\prime}}{b-a}\left(1-\psi\left(\bar{x}^{s}\right)\right)-\left(M_{b}^{\prime} \frac{\bar{x}^{s}-a}{b-a}+M_{a}^{\prime} \frac{b-\bar{x}^{s}}{b-a}\right) \psi^{\prime}\left(\bar{x}^{s}\right) \\
& -\frac{\psi^{\prime}\left(\bar{x}^{s}\right)}{\left(1-c_{1}\right)(b-a)}\left[\left(M_{b}^{\prime}+M_{a}^{\prime}\right) \frac{b-a}{2}-\int_{a}^{b}\left(M_{b}^{\prime} \frac{z-a}{b-a}+M_{a}^{\prime} \frac{b-z}{b-a}\right) \psi(z) d z\right] \\
= & \frac{\bar{\psi}^{\prime}\left(\bar{x}^{s}\right)}{\left(1-c_{1}\right)(b-a)}\left[\frac{M_{b}-M_{a}}{b-a}-\frac{M_{b}^{\prime}+M_{a}^{\prime}}{2}\right]+\frac{M_{b}^{\prime}-M_{a}^{\prime}}{b-a}\left(1-\psi\left(\bar{x}^{s}\right)\right) \\
& +\frac{\bar{\psi}^{\prime}\left(\bar{x}^{s}\right)}{b-a}\left[\int_{a}^{b}\left(M_{b}^{\prime} \frac{z-a}{b-a}+M_{a}^{\prime} \frac{b-z}{b-a}\right) \frac{\psi(z)}{\left(1-c_{1}\right)(b-a)} d z-\left(M_{b}^{\prime} \frac{\bar{x}^{s}-a}{b-a}+M_{a}^{\prime} \frac{b-\bar{x}^{s}}{b-a}\right)\right] \\
= & \text { (A) }+ \text { (B) }+ \text { (C). }
\end{aligned}
$$

To estimate (A), we use Lemma 6.7 to conclude that both $M_{a}^{\prime}$ and $M_{b}^{\prime}$ are close to $\frac{M_{b}-M_{a}}{b-a}$, so that

$$
|A| \leq \frac{\left|\bar{\psi}^{\prime}\right|}{\left(1-c_{1}\right)(b-a)} 2 C \rho^{-31 / 105}|a-b|^{1 / 7} \leq C^{\prime} \rho^{-31 / 105}|a-b|^{-6 / 7} .
$$

For (B), we again use that $\left|M_{b}^{\prime}-M_{a}^{\prime}\right| \leq 2 C \rho^{-31 / 105}|a-b|^{1 / 7}$ by Lemma 6.7, so that

$$
|\mathrm{B}| \leq C^{\prime} \rho^{-31 / 105}|a-b|^{-6 / 7} \text {. }
$$

Finally, to estimate (C), note that the first term inside the brackets is the average value of $M_{b}^{\prime} \frac{z-a}{b-a}+M_{a}^{\prime} \frac{b-z}{b-a}$ with respect to the smooth probability measure having density $\frac{\psi}{\left(1-c_{1}\right)(b-a)}$. Thus there exists $\bar{y}^{s} \in[a, b]$ such that this average value equals the function value at $\bar{y}^{s}$.

$$
\begin{aligned}
|\mathrm{C}| & =\frac{\left|\bar{\psi}^{\prime}\right|}{b-a}\left|M_{b}^{\prime} \frac{\bar{y}^{s}-a}{b-a}+M_{a}^{\prime} \frac{b-\bar{y}^{s}}{b-a}-\left(M_{b}^{\prime} \frac{\bar{x}^{s}-a}{b-a}+M_{a}^{\prime} \frac{b-\bar{x}^{s}}{b-a}\right)\right| \\
& =\frac{\left|\psi^{\prime}\right|}{b-a} \frac{\left|\bar{y}^{s}-\bar{x}^{s}\right|}{b-a}\left|M_{b}^{\prime}-M_{a}^{\prime}\right| \leq C^{\prime} \rho^{-31 / 105}|a-b|^{-6 / 7},
\end{aligned}
$$


where we have again used Lemma 6.7 to bound the difference $\left|M_{b}^{\prime}-M_{a}^{\prime}\right|$. Collecting our estimates for (A), (B) and (C), we have the following bound,

$$
\left|\partial_{\bar{x}^{s}} \partial_{\bar{x}^{s}} \sigma\left(\bar{x}^{u}, \bar{x}^{s}\right)\right| \leq C \rho^{-31 / 105}|a-b|^{-6 / 7} \text {. }
$$

Now we return to the four-point estimate for $\partial_{\bar{x}^{s}} \sigma$. Grouping the terms according to their unstable coordinates and using (6.19), we estimate,

$$
\begin{aligned}
\left|\partial_{\bar{x}^{s}} \sigma\left(\bar{x}^{u}, \bar{x}^{s}\right)-\partial_{\bar{x}^{s}} \sigma\left(\bar{x}^{u}, \bar{y}^{s}\right)-\partial_{\bar{x}^{s}} \sigma\left(\bar{y}^{u}, \bar{x}^{s}\right)+\partial_{\bar{x}^{s}} \sigma\left(\bar{y}^{u}, \bar{y}^{s}\right)\right| & \leq C \rho^{-31 / 105}\left|\bar{y}^{s}-\bar{x}^{s}\right||a-b|^{-6 / 7} \\
& \leq C \rho^{-31 / 105}\left|\bar{y}^{s}-\bar{x}^{s}\right|^{1 / 7} .
\end{aligned}
$$

Putting this estimate together with (6.18), we see that the four-point difference is bounded by the minimum of these two quantities; let us call them $X$ and $Y$. But if a quantity $Q$ satisfies $0 \leq Q \leq X$ and $0 \leq Q \leq Y$, then

$$
Q=Q^{1-7 \varpi} Q^{7 \varpi} \leq X^{1-7 \varpi} Y^{7 \varpi}
$$

provided $0 \leq 7 \varpi \leq 1$. Using this we obtain

$$
\begin{aligned}
& \left|\partial_{\bar{x}^{s}} \sigma\left(\bar{x}^{u}, \bar{x}^{s}\right)-\partial_{\bar{x}^{s}} \sigma\left(\bar{x}^{u}, \bar{y}^{s}\right)-\partial_{\bar{x}^{s}} \sigma\left(\bar{y}^{u}, \bar{x}^{s}\right)+\partial_{\bar{x}^{s}} \sigma\left(\bar{y}^{u}, \bar{y}^{s}\right)\right| \\
& \leq C k_{W}^{-4 / 3+28 \varpi / 3} \rho^{-2 / 3+13 \varpi / 5}\left|\bar{y}^{u}-\bar{x}^{u}\right|^{1-7 \varpi}\left|\bar{y}^{s}-\bar{x}^{s}\right|^{\varpi},
\end{aligned}
$$

which completes the required four-point estimate in the gap for the foliation on the scatterer.

Up to this point, we have proved the four-point estimate both in the gaps in the current subsection and within each interval containing a surviving part of the foliation in Lemma 6.6. However, this is not sufficient to prove a uniform four-point estimate along the whole of $P^{+}(W)$ since, given two stable coordinates $\bar{x}^{s}, \bar{y}^{s}$ with multiple gaps between them, we would have to apply the triangle inequality once for each gap. Since the number of gaps grows exponentially with $n$, the Hölder control established by the four-point estimate would not extend uniformly across $P^{+}(W)$. To remedy this situation, our next lemma proves a bound analogous to Lemma 6.6 across gaps in the surviving foliation.

Lemma 6.8 (Hölder continuity across gaps). Let $P^{+}(W)$ be a stable curve parametrized by $\bar{x}^{s} \in\left[a^{\prime}, b^{\prime}\right]$ as above and suppose the interval $(a, b) \subset\left[a^{\prime}, b^{\prime}\right]$ contains one or more gaps in the surviving foliation pushed forward $n$ steps. Fix $C^{\prime}>0$ and consider the set of stable curves $V_{1}, V_{2} \in \mathcal{W}^{s}$ which are connected by the surviving foliation on either side of the gap such that the angles between $V_{1}$ and $V_{2}$ satisfy $\phi\left(x, \mathbf{h}_{12}(x)\right) \leq C^{\prime} d\left(x, \mathbf{h}_{12}(x)\right)$ for all $x \in V_{1}$, where $\mathbf{h}_{12}$ denotes the holonomy from $V_{1}$ to $V_{2}$. We require that $P^{+}(W)$ be in this set. For any $0 \leq \varpi \leq 1 / 20$, 31 there exists $C>0$, independent of $W$ and $n$, such that for any two such curves $V_{1}, V_{2}$ and two points, $x_{a}=\left(\bar{G}\left(x^{u}, a\right), a\right), x_{b}=\left(\bar{G}\left(x^{u}, b\right), b\right) \in V_{1}$, we have

$$
\ln \frac{J \mathbf{h}_{12}\left(x_{a}\right)}{J \mathbf{h}_{12}\left(x_{b}\right)} \leq C k_{W}^{-2} \rho^{-4 / 5-11 \varpi / 15} d\left(x_{a}, \mathbf{h}_{12}\left(x_{a}\right)\right)^{1-\varpi}|a-b|^{\varpi} .
$$

Proof. Using similar notation to Subsection 6.3 (and the proof of Lemma 6.7), let $j+1$ denote the least integer $j^{\prime} \geq 1$ such that an element of $T^{j^{\prime}}\left(\mathcal{S}_{0}\right)$ intersects $P^{+}(W)$ in the subcurve defined by $(a, b)$. This implies in particular, that the surviving parts of the foliation containing $x_{a}$ and $x_{b}$ lie in the same homogeneity strip for the first $j$ interates of $T^{-1}$. Let $V_{i}^{j}=T^{-j} V_{i}, i=1,2$, and let $\mathbf{h}_{-j}$ denote the holonomy map from $V_{1}^{j}$ to $V_{2}^{j}$. Then,

$$
\ln \frac{J \mathbf{h}_{12}\left(x_{a}\right)}{J \mathbf{h}_{12}\left(x_{b}\right)}=\ln \frac{J_{V_{1}}^{s} T^{-j}\left(x_{a}\right)}{J_{V_{2}}^{s} T^{-j}\left(\mathbf{h}_{12}\left(x_{a}\right)\right)}-\ln \frac{J_{V_{1}}^{s} T^{-j}\left(x_{b}\right)}{J_{V_{2}}^{s} T^{-j}\left(\mathbf{h}_{12}\left(x_{b}\right)\right)}+\ln \frac{J \mathbf{h}_{-j}\left(T^{-j} x_{a}\right)}{J \mathbf{h}_{-j}\left(T^{-j} x_{b}\right)} .
$$

\footnotetext{
${ }^{31}$ Equation (6.23) below shows that we can get away with $\varpi \leq 1 / 15$, up to using a more complicated expression for the upper bound.
} 
Since $T^{-i}\left(x_{a}\right)$ and $T^{-i}\left(x_{b}\right)$ lie in the same homogeneity strip for $i=0, \ldots j$, the difference of the first two terms in (6.21) can be estimated in precisely the same way as the difference of Jacobians in (A.1) in the proof of Lemma 6.6. Thus fixing $\varpi \leq 1 / 15$, there exists $C>0$ such that

$$
\begin{aligned}
& \left|\ln \frac{J_{V_{1}}^{s} T^{-j}\left(x_{a}\right)}{J_{V_{2}}^{s} T^{-j}\left(\mathbf{h}_{12}\left(x_{a}\right)\right)}-\ln \frac{J_{V_{1}}^{s} T^{-j}\left(x_{b}\right)}{J_{V_{2}}^{s} T^{-j}\left(\mathbf{h}_{12}\left(x_{b}\right)\right)}\right| \\
& \quad \leq C|a-b|^{\varpi} d\left(x_{a}, \mathbf{h}_{12}\left(x_{a}\right)\right)^{1-\varpi} \max \left\{k_{W}^{-4 / 3-10 \varpi / 3} \rho^{-2(1+\varpi) / 3}, k_{W}^{-82 \varpi / 3} \rho^{-38 \varpi / 3}\right\} .
\end{aligned}
$$

Next we estimate $\ln J \mathbf{h}_{-j}\left(T^{-j} x_{a}\right)$ using (A.11). Let $x_{j}=T^{-j}\left(x_{a}\right), x_{0}=x_{a}$ and $\tilde{x}_{0}=\mathbf{h}_{12}\left(x_{a}\right)$. Due to (6.22), we cannot use an estimate with a factor better than $|a-b|^{\varpi}$ and since $\varpi \leq 15$, we do not convert the full power of $J_{T^{-j} \bar{\gamma}}^{u} T^{j}\left(x_{j}\right)$ in the denominator of the bound in (A.11) using Sublemma A.1. Rather, we convert $J_{T^{-j} \bar{\gamma}}^{u} T^{j}\left(x_{j}\right)^{35 \varpi / 9}$, after noting that $35 \varpi / 9 \leq 7 / 27<3 / 5$. Thus,

$$
\begin{aligned}
\ln J \mathbf{h}_{-j}\left(x_{j}\right) & \leq C\left(\frac{d\left(x_{0}, \tilde{x}_{0}\right)|a-b|^{\varpi}}{\rho^{2 / 3+26 \varpi / 9} k_{W}^{4 / 3+70 \varpi / 9}}+\frac{d\left(x_{0}, \tilde{x}_{0}\right)|a-b|^{\varpi} k_{W}^{4 / 5}}{\rho^{2 / 5+26 \varpi / 9} k_{W}^{70 \varpi / 9} k_{W}^{6 / 5-70 \varpi / 9}}\right) \\
& \leq C d\left(x_{0}, \tilde{x}_{0}\right)^{1-\varpi}|a-b|^{\varpi}\left(k_{W}^{-4 / 3-52 \varpi / 9} \rho^{-2 / 3-17 \varpi / 9}+k_{W}^{-2 / 5+2 \varpi} \rho^{-2 / 5-17 \varpi / 9}\right),
\end{aligned}
$$

where in the second term on the first line we have converted the remaining power of $J_{T^{-j} \bar{\gamma}}^{u} T^{j}\left(x_{j}\right)$ to $k_{W}^{6 / 5-70 \varpi / 9}$ to cancel the power of $k_{W}$ in the numerator of that fraction; and in the second line we have used $d\left(x_{0}, \tilde{x}_{0}\right) \leq k_{W}^{2} \rho$. A similar estimate holds for $\ln J \mathbf{h}_{-j}\left(T^{-j}\left(x_{b}\right)\right)$. This estimate together with (6.22) in (6.21) yields four factors involving $k_{W}$ and $\rho$. In anticipation of Subsection 6.5 and to simplify these terms, we factor out $k_{W}^{-2}$ from each term and convert the remaining powers of $k_{W}$ using the inequality, $k_{W} \leq \rho^{-1 / 5}$. The factor involving $\rho$ in our bound for $\ln \frac{J \mathbf{h}_{12}\left(x_{a}\right)}{J \mathbf{h}_{12}\left(x_{b}\right)}$ is then,

$$
\max \left\{\rho^{-2 / 5-36 \varpi / 5}, \rho^{-4 / 5-11 \varpi / 15}, \rho^{-18 / 25-103 \varpi / 45}\right\},
$$

where we have dropped one of the terms, $\rho^{-4 / 5}$, as being clearly less than the middle term above. Unfortunately, the remaining three exponents intersect for $\varpi<1 / 15$, so there is no clear maximum in this range; however, if we restrict to $0<\varpi \leq 1 / 20$, the middle factor is the largest so we may drop the other two, completing the proof of Lemma 6.8.

Finally, we use Lemma 6.8 to extend the four-point estimate uniformly across gaps along the whole of $P^{+}(W)$. If we are given two stable coordinates $\bar{x}^{s}, \bar{y}^{s}$ corresponding to surviving parts of the foliation between which there may be multiple gaps and multiple bits of surviving foliation, Lemma 6.8 immediately implies the four-point estimate holds between $\bar{x}^{s}$ and $\bar{y}^{s}$. Now suppose $\bar{x}^{s}$ and $\bar{y}^{s}$ belong to different gaps in which the foliation has been interpolated. Then using (6.20) we have a four-point estimate from $\bar{x}^{s}$ to the edge of its gap closest to $\bar{y}^{s}$, call that stable coordinate $a$; similarly, the four-point estimate holds from $\bar{y}^{s}$ to the edge of its gap closest to $\bar{x}^{s}$, call this stable coordinate $b$. Now from $a$ to $b$ there may be multiple gaps, but by Lemma 6.8, the four-point estimate holds from $a$ to $b$ and so by the triangle inequality, it extends from $\bar{x}^{s}$ to $\bar{y}^{s}$. Notice that decomposing the distance from $\bar{x}^{s}$ to $\bar{y}^{s}$ in this way, we only have to add three terms, using the triangle inequality twice, making the estimate uniform on all of $P^{+}(W)$. Similarly, we need only use the triangle inequality once to obtain the four-point estimate given $\bar{x}^{s}$ in a gap and $\bar{y}^{s}$ in a surviving piece of foliation or vice versa. 
6.5. Lifting the map-foliation to a flow foliation, checking (i-viii). In this step, we lift the map foliation $\{\bar{\gamma}\}$ to $W$.

Given a curve $\bar{\gamma}$ in our foliation, we want to define a "lift" of $\bar{\gamma}$ to a curve $\gamma \in \mathcal{W}^{u}$ which intersects $W$ in a point and such that $P^{+}(\gamma)=\bar{\gamma}$. Adopting the coordinates $\left(x^{u}, x^{s}, x^{0}\right)$ defined by Remark 2.4, we want to find a parametrisation $\gamma(v)=\left(x^{u}(v), x^{s}(v), x^{0}(v)\right)$, with $v \in I$, an interval, which has the desired properties. Note that the functions $x^{u}(v)$ and $x^{s}(v)$ are uniquely determined by the projection $P^{+}(W)$ (they do not depend on $x^{0}$ ) so the only degree of freedom is in $x^{0}(v)$. However, since $\gamma$ must lie in the kernel of the contact form, we must have $\mathbf{d} x^{0}-x^{s} \mathbf{d} x^{u}=0$, i.e., $\left(x^{0}\right)^{\prime}=\left(x^{u}\right)^{\prime} \cdot x^{s}$. This, together with the initial condition that $x^{0}(0)$ must be an endpoint of $\gamma$ uniquely determines $\gamma$.

Now according to Lemma 3.4 the map from $P^{+}(W)$ to $W$ is $\mathcal{C}^{1+1 / 2}$ with uniformly bounded norm (not depending on $k_{W}$ ). Thus the measure of $\bar{\Delta}_{\varkappa}$ in $P^{+}(W)$ is comparable to the measure of $\Delta_{\varkappa}$ in $W$, proving item (iv). Items (i)-(iii) follow directly from the definition of $\{\gamma\}$ and the construction of $\{\bar{\gamma}\}$, recalling (6.5).

In order to determine the smoothness of the lifted foliation, we must consider the action of lifting $\bar{\gamma}$ off the scatterer in the unstable direction as well. In the unstable direction, the curves $\bar{\gamma}$ undergo a contraction on the order of $1 / J_{T^{-1} \bar{\gamma}}^{u} T \approx k_{W}^{-2}$. Using the usual distortion bounds together with the expression for the Jacobian given by (6.4), we see that

$$
\left|\ln \frac{J_{T^{-1} \bar{\gamma}}^{u} T(x)}{J_{T^{-1} \bar{\gamma}}^{u} T(y)}\right| \leq C d(x, y) k_{W}^{2},
$$

for any $x, y \in \bar{\gamma}$ and a similar estimate holds between different curves by estimates similar to those used in the proof of Lemma 6.4. Thus the Lipschitz constants of the foliation increase by a factor of $k_{W}^{2} \leq C \rho^{-2 / 5}$.

For $\bar{\gamma}$ on the domain $\bar{\Delta}_{\varkappa}$, we have $\left|\partial_{\bar{x}^{u}} \partial_{\bar{x}^{s}} \bar{G}\right| \leq C \rho^{-2 / 3} k_{W}^{-2}$ by Section 6.2 , so that $\left|\partial_{x^{u}} \partial_{x^{s}} G\right| \leq$ $C \rho^{-2 / 3}$. On the complement of the domain $\bar{\Delta}_{\varkappa}$, the Lipschitz constant of the projected foliation is bounded by $C+C k_{W}^{-4 / 3} \rho^{-2 / 3}$ by (6.16) in Section 6.3. Thus the Lipschitz constant of the lifted foliation is bounded by $C k_{W}^{2}+C k_{W}^{2 / 3} \rho^{-2 / 3} \leq C \rho^{-4 / 5}$, which yields item (vi) of the foliation. We can then integrate $\partial_{x^{u}} \partial_{x^{s}} G$ to obtain the uniform bound on $\partial_{x^{s}} G$ needed for item (v) of the foliation, recalling (ii).

Finally, using (6.20) and Lemmas 6.6 and 6.8, and collecting the relevant terms, we see that the constant in the right-hand side of the four-point condition (vii) for the Jacobian is at most

$$
\max \left\{\rho^{-4 / 5}, \rho^{-2 / 5-36 \varpi / 5}, \rho^{-4 / 5+37 \varpi / 15}, \rho^{-4 / 5-11 \varpi / 15}\right\} .
$$

If we restrict to $\varpi \leq 1 / 20$, the last term above is the largest, completing the proof of (vii).

Finally, we turn to condition (viii). Note that since $\gamma$ lies in the kernel of the contact form,

we have $H\left(x^{u}, x^{s}\right)=\int_{0}^{x^{u}} G\left(z, x^{s}\right) d z$. In particular, $\partial_{x^{s}} H\left(x^{u}, x^{s}\right)=\int_{0}^{x^{u}} \partial_{x^{s}} G\left(z, x^{s}\right) d z$, so that since $\partial_{x^{s}} G$ uniformly bounded, we have $\left|\partial_{x^{s}} H\right|_{C^{0}} \leq C \rho$.

Next, we note that due to the normalization $W=\left(0, x^{s}, 0\right)$, we have $\partial_{x^{s}} G\left(0, x^{s}\right)=1$ for all $x^{s} \in[0,|W|]$. Thus using the four-point estimate (vii) for $G$ at the points $\left(x^{u}, x^{s}\right),\left(x^{u}, y^{s}\right)$, $\left(0, x^{s}\right)$ and $\left(0, y^{s}\right)$, we have

$$
\left|\partial_{x^{s}} G\left(x^{u}, x^{s}\right)-\partial_{x^{s}} G\left(x^{u}, y^{s}\right)\right| \leq C \rho^{-4 / 5-11 \varpi / 15}\left|x^{s}-y^{s}\right|^{\varpi}\left|x^{u}\right|^{1-7 \varpi} .
$$

This implies immediately that $\left|\partial_{x^{s}} H\right|_{\mathcal{C}^{\varpi}} \leq C \rho^{6 / 5-116 \varpi / 15}$, which completes the proof of (viii).

\section{Mollification OPERATORS AND EMBEDDingS}

In this section we prove some relations between our Banach spaces and standard spaces of distributions, and establish several key inequalities which indicate that mollification operators 
provide good approximations in the norms we have defined. We begin by proving Lemma 2.11 . relating our norms to the dual spaces of continuous functions.

Proof of Lemma 2.11. We will prove that

$$
|f|_{\left(\mathcal{C}^{\alpha}\left(\Omega_{0}\right)\right)^{*}} \leq C|f|_{w} \quad \forall f \in \mathcal{C}^{0}\left(\Omega_{0}\right)
$$

for some $C>0$. The analogous inequality for the strong norm, $|f|_{\left(\mathcal{C}^{\beta}\left(\Omega_{0}\right)\right)^{*}} \leq C\|f\|_{s}$, is proved similarly.

For $f \in \mathcal{C}^{0}\left(\Omega_{0}\right)$ and $\psi \in \mathcal{C}^{\alpha}\left(\Omega_{0}\right)$ with $|\psi|_{\mathcal{C}^{\alpha}\left(\Omega_{0}\right)} \leq 1$, we must estimate $\int_{\Omega_{0}} f \psi d m$. To estimate this, we decompose Lebesgue measure over the Poincare section $\mathcal{M}$ defined in Section 2 ,

Our first step is to decompose $\mathcal{M}$ into boxes foliated by a smooth family of homogeneous map-stable curves. (For the present lemma, it would be enough to consider a smooth family of map-stable curves, the further decomposition into homogeneous curves is useful in view of the proof of Lemma 7.3 below.) On each connected component $\mathbb{H}_{k, i}$ of $\mathbb{H}_{k}, k>k_{0}$, we define a smooth foliation $\left\{V_{\xi}\right\}_{\xi \in E_{k, i}}$ of map-stable curves; indeed, we may choose a foliation of straight line segments due to the global stable cones for the map. Using this foliation, we desintegrate the probability measure $\bar{m}=\bar{c} d r d \varphi$ into $c^{\prime} d \bar{m}_{V_{\xi}} d \xi$, where $c^{\prime}$ is a constant depending smoothly on the angle between the foliation of stable curves and the boundary of $\mathbb{H}_{k}$, and $\bar{m}_{V}$ is arclength measure on the curve $V$. On the set $\mathbb{H}_{k_{0}}:=\mathcal{M} \backslash \cup_{k>k_{0}} \mathbb{H}_{k}$, we perform a similar decomposition, after first subdividing the space into boxes $B_{i}$ which are foliated by parallel stable line segments of length at most $L_{0}$.

Next, we lift this decomposition to $\Omega_{0}$. For $k>k_{0}$, define

$$
H_{k}^{0-}=\left\{Y \in \Omega_{0}: P^{+}(Y) \in \mathbb{H}_{k}\right\},
$$

and $H_{k_{0}}^{0-}=\Omega_{0} \backslash \cup_{k>k_{0}} H_{k}^{0-}$. Over each box $B_{i}$ define the flow region $B_{i}^{0-}=\left\{Z \in \Omega_{0}: P^{+}(Z) \in\right.$ $\left.B_{i}\right\}$.

In each $B_{i}^{0-}$ or $H_{k}^{0-}$, we represent Lebesgue measure as $32 \cos \varphi d s d r d \varphi$, where $r, \varphi$ range over the box $B_{i}$ or strip $\mathbb{H}_{k}$ and $s$ ranges from 0 to the maximum free flight time under the backwards flow of any point in the box $B_{i}$, which we denote by $\tau_{\max , i}^{-}$. For each $s$ in this range and each curve $V_{\xi}$, let $W_{\xi}^{s}=\Phi_{-t(s)}\left(V_{\xi}\right)$, where the function $t(s, z)$ is defined for $z \in V_{\xi}$ so that $W_{\xi}^{s}$ lies in the kernel of the contact form. Note that for $s<L_{0}$, it may be that some points in $V_{\xi}$ have not yet lifted off of $\mathcal{M}$. For such small times, $W_{\xi}^{s}$ denotes only those points which have lifted off of $\mathcal{M}$ and so may be the union of at most 33 two flow-stable curves. Also, for $s>\tau_{\text {min }}$, it may be that part of $\Phi_{-t(s)}\left(V_{\xi}\right)$ has made a collision with a scatterer. In this case, again, $W_{\xi}^{s}$ denotes only those points which have not yet made a first collision. Thus, using the disintegration of $\bar{m}$ described above, $c \cos \varphi d s d r d \varphi=\cos \varphi\left(P^{+} Z\right) \rho_{\xi}(Z) d m_{W_{\xi}^{s}}(Z) d \xi d s$, where $\xi \in E_{k, i}$ and (using that the disintegration factor $c^{\prime}$ from the third paragraph of the proof is smooth) $\left|\rho_{\xi}\right|_{\mathcal{C}^{1}\left(W_{\xi}^{s}\right)} \leq C$, uniformly in $\xi$ and $s$. Now,

$$
\begin{aligned}
\left|\int_{\Omega_{0}} f \psi d m\right| & =\left|\sum_{k \geq k_{0}} \sum_{i} \int_{0}^{\tau_{\max , i}^{-}} \int_{E_{k, i}} \int_{W_{\xi}^{s}} f \psi \rho_{\xi} \cos \varphi\left(P^{+}\right) d m_{W_{\xi}^{s}} d \xi d s\right| \\
& \leq \sum_{k \geq k_{0}} \sum_{i} \int_{0}^{\tau_{\max , i}^{-}} \int_{E_{k, i}}|f|_{w}\left|\rho_{\xi}\right|_{\mathcal{C}^{\alpha}\left(W_{\xi}^{s}\right)}\left|\cos \varphi\left(P^{+}\right)\right|_{\mathcal{C}^{\alpha}\left(W_{\xi}^{s}\right)}|\psi|_{\mathcal{C}^{\alpha}\left(\Omega_{0}\right)} d \xi d s .
\end{aligned}
$$

\footnotetext{
32 The projection on the scatterers of Lebesgue measure is the $T$-invariant probability measure $\mu_{0}=$ $c \cos \varphi d r d \varphi$, where $c$ is a normalization constant.

33 Just like for (2.5), see the paragraph containing (8.6).
} 
Now $|\cos \varphi|_{\mathcal{C}^{0}\left(\mathbb{H}_{k}\right)} \leq C k^{-2}$, while for $y, z \in V_{\xi} \subset \mathbb{H}_{k}$,

$$
|\cos \varphi(y)-\cos \varphi(z)| \leq|y-z|^{\alpha}|y-z|^{1-\alpha} \leq|y-z|^{\alpha} C k^{-3(1-\alpha)} .
$$

Since $\alpha \leq 1 / 3$, the Hölder constant of $\cos \varphi$ is bounded by $C k^{-2}$ as well. Since $P^{+}$is $\mathcal{C}^{1}$ along stable curves by Lemma 3.4, we have $\left|\cos \varphi\left(P^{+}\right)\right|_{\mathcal{C}^{\alpha}\left(W_{\xi}^{s}\right)} \leq C k^{-2}$, independently of $\xi$ and $s$. Also, for each $k \geq k_{0}$, the number of boxes $B_{i}$ in $\mathbb{H}_{k}$ is finite, depending on $L_{0}$. Thus,

$$
\left|\int_{\Omega_{0}} f \psi d m\right| \leq \sum_{k} C \tau_{\max }|f|_{w} k^{-2} \leq C^{\prime}|f|_{w}
$$

completing the proof of the lemma.

Lemma 7.1. If $\beta<1 / q$, the inclusions $\mathcal{B} \subset\left(\mathcal{C}^{\beta}\left(\Omega_{0}\right)\right)^{*}$ and $\mathcal{B} \subset\left(\mathcal{C}^{1}\left(\Omega_{0}\right)\right)^{*}$ are injective.

Proof. Our proof has two steps: (1) for $f \in \mathcal{B}$, if $\|f\|_{\mathcal{B}} \neq 0$, then $\|f\|_{s} \neq 0$; (2) if $\|f\|_{s} \neq 0$, then $f \neq 0$ as an element of $\left(\mathcal{C}^{\beta}(M)\right)^{*}$.

For claim (1), note that $\|f\|_{s}=0$ implies immediately that $\|f\|_{u}=0$ since the test functions for $\|\cdot\|_{u}$ are in $\mathcal{C}^{\alpha}(W)$, while those for $\|\cdot\|_{s}$ are in $\mathcal{C}^{\beta}(W)$ and $\alpha>\beta$. To see that $\|f\|_{0}=0$ as well, observe that for fixed $W \in \mathcal{W}^{s}, \psi \in \mathcal{C}^{\alpha}(W)$, the functional $F_{s}(f)=\int_{\Phi_{-s}(W)} \frac{d}{d r}(f \circ$ $\left.\Phi_{r}\right)\left.\right|_{r=0} \psi \circ \Phi_{s} J_{\Phi_{-s}(W)} \Phi_{s} d m_{\Phi_{-s}(W)}$ is continuous as a function of $s$ as long as $\Phi_{-s}(W)$ undergoes no collisions. (It is clearly continuous for $f \in \mathcal{C}^{2}\left(\Omega_{0}\right)$ and extends to $f \in \mathcal{B}$ by density since the map $f \rightarrow F_{s}(f)$ is continuous in the $\|\cdot\|_{\mathcal{B}}$ norm.) Thus

$$
\left.\lim _{t \downarrow 0} \frac{1}{t} \int_{0}^{t} \int_{\Phi_{-s}(W)} \frac{d}{d r}\left(f \circ \Phi_{r}\right)\right|_{r=0} \psi \circ \Phi_{s} J_{\Phi_{-s}(W)} \Phi_{s} d m_{\Phi_{-s}(W)} d s=\left.\int_{W} \frac{d}{d r}\left(f \circ \Phi_{r}\right)\right|_{r=0} \psi d m_{W} .
$$

On the other hand,

$$
\begin{gathered}
\left.\int_{0}^{t} \int_{\Phi_{-s}(W)} \frac{d}{d r}\left(f \circ \Phi_{r}\right)\right|_{r=0} \psi \circ \Phi_{s} J_{\Phi_{-s}(W)} \Phi_{s} d m_{\Phi_{-s}(W)} d s=\left.\int_{W} \int_{0}^{t} \frac{d}{d r}\left(f \circ \Phi_{r}\right)\right|_{r=0} \circ \Phi_{-s} \psi d s d m_{W} \\
=\int_{W} \int_{0}^{t} \frac{d}{d s}\left(f \circ \Phi_{-s}\right) \psi d s d m_{W}=\int_{\Phi_{-t}(W)} f \psi \circ \Phi_{t} J_{\Phi_{-t}(W)} \Phi_{t}-\int_{W} f \psi=0,
\end{gathered}
$$

by assumption on $f$, where we have used (2.11) for the second equality. Thus $\|f\|_{0}=0$ as claimed.

Our proof of claim (2) follows closely [DZ3, Lemma 3.8]. For $f \in \mathcal{C}^{2}\left(\Omega_{0}\right)$ and $W \in \mathcal{W}^{s}$, the expression,

$$
\left\langle D_{W}^{\beta}(f), \psi\right\rangle=\int_{W} f \psi d m_{W}, \quad \psi \in \mathcal{C}^{\beta}\left(\Omega_{0}\right),
$$

satisfies $\left|\left\langle D_{W}^{\beta}(f), \psi\right\rangle\right| \leq\|f\|_{s}|W|^{1 / q} \mid \psi_{\mathcal{C}^{\beta}(W)}$, so that $D_{W}^{\beta}(f) \in\left(\mathcal{C}^{\beta}\left(\Omega_{0}\right)\right)^{*}$. Since the map $f \rightarrow$ $D_{W}^{\beta}(f)$ is continuous in the $\|\cdot\|_{\mathcal{B}}$ norm, by density it can be extended to $\mathcal{B}$.

Now assume $\|f\|_{s} \neq 0$. There exists $W \in \mathcal{W}^{s}, \psi \in \mathcal{C}^{\beta}\left(\Omega_{0}\right)$ such that $\left\langle D_{W}^{\beta}(f), \psi\right\rangle=: \delta>0$. Again, the map $W \rightarrow\left\langle D_{W}^{\beta}(f), \psi\right\rangle$ is continuous for $f \in \mathcal{B}$. Thus there exists an open set $E$, foliated by invariant curves $W^{\prime} \in \mathcal{W}^{s}$ close to $W$ such that $\left\langle D_{W^{\prime}}^{\beta}(f), \psi\right\rangle \geq \delta / 2$ for each $W^{\prime} \subset E$.

In order to localize the support of $\psi$ to the set $E$, we extend each stable curve $W^{\prime}$ in $E$ by length $\varepsilon>0$ at both ends to form a larger set $E^{\prime} \supset E$. Call such extended curves $W_{\varepsilon}^{\prime}$. Next we choose a bump function $\rho_{\varepsilon}$ such that $\rho_{\varepsilon}=0$ on $\Omega_{0} \backslash E^{\prime}$ and $\rho_{\varepsilon}=1$ on $E$. We may choose $\rho_{\varepsilon}$ so that $\left|\rho_{\varepsilon}\right|_{\mathcal{C}^{\beta}\left(W_{\varepsilon}^{\prime}\right)} \leq C \varepsilon^{-\beta}$ for some uniform constant $C$. Now,

$$
\begin{aligned}
\left\langle D_{W_{\varepsilon}^{\prime}}^{\beta}(f), \rho_{\varepsilon} \psi\right\rangle & =\left\langle D_{W^{\prime}}^{\beta}(f), \rho_{\varepsilon} \psi\right\rangle+\left\langle D_{W_{\varepsilon}^{\prime} \backslash W^{\prime}}^{\beta}(f), \rho_{\varepsilon} \psi\right\rangle \\
& \geq \delta / 2-C|\psi|_{\mathcal{C}^{\beta}\left(\Omega_{0}\right)} \varepsilon^{-\beta}\left|W_{\varepsilon}^{\prime} \backslash W^{\prime}\right|^{1 / q}\|f\|_{s} \geq \delta / 2-C|\psi|_{\mathcal{C}^{\beta}\left(\Omega_{0}\right)}\|f\|_{s} \varepsilon^{1 / q-\beta} .
\end{aligned}
$$


This difference can be made larger than $\delta / 3$ by choosing $\varepsilon$ small since $1 / q<\beta$. Thus the function $\rho_{\varepsilon} \psi \in \mathcal{C}^{\beta}\left(\Omega_{0}\right)$ satisfies $f\left(\rho_{\varepsilon} \psi\right) \neq 0$ and so $f \neq 0$ as an element of $\left(\mathcal{C}^{\beta}\left(\Omega_{0}\right)\right)^{*}$.

The injectivity of $\mathcal{B} \subset\left(\mathcal{C}^{1}\left(\Omega_{0}\right)\right)^{*}$ follows by a similar argument since we may take $\psi \in \mathcal{C}^{1}\left(\Omega_{0}\right)$ in the proof of claim (2). This holds since we have defined $\mathcal{C}^{\beta}(W)$ to be the closure of $\mathcal{C}^{1}$ functions in the $\mathcal{C}^{\beta}$ norm.

7.1. Mollification operators. Since the right-hand side of the Dolgopyat Lemma 8.1 will involve the Lipschitz and supremum norm of $f$, it will be convenient to use mollification operators $\mathbb{M}_{\epsilon}$.

We start by defining $\mathbb{M}_{\epsilon}$ : Fix $\epsilon_{0}$ small 34 Let $\eta: \mathbb{R}^{3} \rightarrow[0, \infty)$ be a $\mathcal{C}^{\infty}$ function, supported in $|Y| \leq 1$ and bounded away from zero on $|Y| \leq 1 / 3$, with $\int \eta d m=1$, and set, for $0<\epsilon<\epsilon_{0}$,

$$
\eta_{\epsilon}(Y)=\frac{1}{\epsilon^{3}} \eta\left(\frac{Y}{\epsilon}\right) \text {. }
$$

Let $\Omega_{1}$ be an $\epsilon_{0}$ neighborhood of $\Omega_{0}$, assuming that $\epsilon_{0}$ is small enough so that $\Omega_{1}$ is still in the torus.

Definition 7.2 (The mollifier operator $\mathbb{M}_{\epsilon}$ ). Fix global periodic coordinates on $\mathbb{T}^{3}$, i.e., view $\mathbb{T}^{3}$ as a subset of $\mathbb{R}^{3}$, extending functions periodically. For $0<\epsilon<\epsilon_{0}$, set for $f \in L^{\infty}\left(\Omega_{0}\right)$

$$
\mathbb{M}_{\epsilon}(f)(Z)=\int_{\mathbb{R}^{3}} \eta_{\epsilon}(Z-Y) f(Y) d m(Y)=\left[\eta_{\epsilon} * f\right](Z) .
$$

Since $f$ is supported in $\Omega_{0}$, we have that $\mathbb{M}_{\epsilon}(f)$ is supported in a small neighborhood of $\Omega_{0}$ contained in $\Omega_{1}$. For $f \in \mathcal{C}^{1}\left(\mathbb{T}^{3}\right)$, not necessarily supported in $\Omega_{0}$, we let

$$
|f|_{H_{\infty}^{1}\left(\Omega_{0}\right)}:=|\nabla(f)|_{L^{\infty}\left(\Omega_{0}\right)} \leq|f|_{H_{\infty}^{1}\left(\mathbb{T}^{3}\right)}:=|\nabla(f)|_{L^{\infty}\left(\mathbb{T}^{3}\right)} .
$$

We have the following bounds for $\mathbb{M}_{\epsilon}$ :

Lemma 7.3. There exists $C$ so that for all small enough $\epsilon>0$, all $\beta$ and all $q$, every admissible stable curve and every $f \in \mathcal{C}^{0}\left(\mathbb{T}^{3}\right)$, supported in $\Omega_{0}$,

$$
\left|\mathbb{M}_{\epsilon}(f)\right|_{L^{\infty}\left(\Omega_{0}\right)} \leq C \epsilon^{-\beta-1+1 / q}\|f\|_{s},
$$

and

$$
\left|\mathbb{M}_{\epsilon}(f)\right|_{H_{\infty}^{1}\left(\Omega_{0}\right)} \leq C \epsilon^{-\beta-2+1 / q}\|f\|_{s} .
$$

The following lemma shows that $\mathbb{M}_{\epsilon}$ is in some sense an approximation of the identity.

Lemma 7.4. There exists $C>0$ so that for all small enough $\epsilon>0$, all $\alpha, \beta, \gamma$, and all $f \in \mathcal{C}^{0}\left(\mathbb{T}^{3}\right)$, supported in $\Omega_{0}$

$$
\left|\mathbb{M}_{\epsilon}(f)-f\right|_{w}^{\mathbb{H}} \leq C \epsilon^{\delta}\|f\|_{\mathcal{B}}
$$

where $\delta=\min \{\gamma, 1 /(2 q), 1 / q-2 / 5-\beta\}$, and where the homogeneous weak norm is defined by

$$
|f|_{w}^{\mathbb{H}}:=\sup _{\substack{W \in \mathcal{W}^{s} \\ \text { homogeneous }}} \sup _{\substack{\psi \in \mathcal{C}^{\alpha}(W) \\|\psi|_{\mathcal{C}^{\alpha}(W)} \leq 1}} \int_{W} f \psi d m_{W} .
$$

The last lemma of this section completes the proof of Lemma 3.9, showing that, although we have taken $\mathcal{B}$ to be the closure of $\left\{\mathcal{L}_{t}\left(\mathcal{C}^{2}\left(\Omega_{0}\right) \cap \mathcal{C}_{\sim}^{0}\right) \mid t \geq 0\right\}$, in fact we have $\mathcal{C}^{1}\left(\Omega_{0}\right) \subset \mathcal{B}$ as well.

Lemma 7.5. $\mathcal{C}^{1}\left(\Omega_{0}\right) \subset \mathcal{B}$.

\footnotetext{
${ }^{34}$ In Section 8 we shall take $\epsilon_{0}$ small enough as a function of $n=\lceil c \ln |b|\rceil$.
} 


\subsection{Proofs of Lemmas $7.3-7.5$,}

Proof of Lemma 7.3. We first bound $\left|\mathbb{M}_{\epsilon} f(Z)\right|$ for all $Z \in \Omega_{0}$. Recalling the notation used in the proof of Lemma 2.11, we decompose the $\epsilon$-neighborhood $N_{\epsilon}(Z)$ of $Z$ inside $\Omega_{0}$, into homogeneous stable curves $W_{\xi}^{s}$ over each component of $N_{\epsilon}(Z) \cap \mathbb{H}_{k}^{0-}$ and $N_{\epsilon}(Z) \cap B_{i}^{0-}$. We estimate the relevant integral over one such component at a time, using (7.2),

$$
\begin{gathered}
\int_{B_{i}^{0-}} f(Y) \eta_{\epsilon}(Z-Y) d m(Y)=\int_{0}^{\tau_{\max , i}^{-}} \int_{E_{k}, i} \int_{W_{\xi}^{s}} f \eta_{\epsilon}(Z-\cdot) \rho_{\xi} \cos \varphi\left(P^{+}\right) d m_{W_{\xi}^{s}} d \xi d s \\
\leq C \epsilon k^{-3}\|f\|_{s} \sup _{\xi, s}\left|W_{\xi}^{s} \cap \operatorname{supp}\left(\eta_{\epsilon}(Z-\cdot)\right)\right|^{1 / q}\left|\eta_{\epsilon}\right|_{\mathcal{C}^{\beta}}\left|\cos \varphi\left(P^{+}\right)\right|_{\mathcal{C}^{\beta}\left(W_{\xi}^{s}\right)}\left|\rho_{\xi}\right|_{\mathcal{C}^{\beta}\left(W_{\xi}^{s}\right)},
\end{gathered}
$$

where the factor $\epsilon k^{-3}$ comes from the fact that the support of $\eta_{\epsilon}$ is of order at most $\epsilon$ in the transverse integral $d s$ and of order $k^{-3}$ in the integral $d \xi$. Next, $\left|\cos \varphi\left(P^{+}\right)\right|_{\mathcal{C}^{\beta}\left(W_{\xi}^{s}\right)} \leq C k^{-2}$, using the estimate following (17.2) and the fact that $\beta<1 / 3$. Also, $\left|\eta_{\epsilon}\right|_{\mathcal{C}^{\beta}} \leq C \epsilon^{-3-\beta}$, and as before, $\left|\rho_{\xi}\right|_{\mathcal{C}^{1}} \leq C$. Putting these estimates together yields,

$$
\left|\int_{B_{i}^{0-}} f(Y) \eta_{\epsilon}(Z-Y) d m(Y)\right| \leq C \epsilon^{-2-\beta+1 / q} k^{-5}\|f\|_{s} .
$$

It remains to sum over the relevant $k$ and $i$. Since there are only a finite number of flow boxes $B_{i}^{0-}$, independent of $\epsilon$, this sum is uniformly bounded. Also, $N_{\epsilon}(Z)$ can cross only a uniformly bounded number of the singularity surfaces $S^{0-}=\left\{Y \in \Omega_{0}: \varphi\left(P^{+}(Y)\right)= \pm \frac{\pi}{2}\right\}$ (in fact, no more than $\tau_{\max } / \tau_{\min }$ of them). Thus the only index which may be unbounded or infinite is $k$. Suppose $N_{\epsilon}(W)$ intersects a range of $k$, from $k_{1}$ to $k_{2}$ (note that $k_{2}=\infty$ is allowed). Since the width in the unstable direction of $\mathbb{H}_{k}^{0-}$ is approximately $C k^{-5}$, we must have $\epsilon$ of order $k_{1}^{-4}-k_{2}^{-4}$, which is precisely the bound we obtain summing over the above estimate, yielding one more power of $\epsilon$ and completing the proof of (7.5).

For the $H_{\infty}^{1}$ bound (7.6), we first differentiate once (which produces an extra factor $\epsilon^{-1}$ ) and then proceed in the same way as above.

Proof of Lemma 7.4. Let $W$ be a homogeneous stable curve and let $\psi$ be a function on $W$ with $|\psi|_{C^{\alpha}}(W) \leq 1$.

Let $A$ be a constant to be determined below. We assume for the moment that $P^{+}(W)$ does not lie within a distance of $A \epsilon^{3 / 5}$ of the boundary of any homogeneity strip. We may also assume that $|W| \leq L_{0} / 2$, since otherwise, we may simply subdivide $W$ into two components and perform the estimate on each component separately.

In order to compare $\mathbb{M}_{\epsilon}(f)$ with $f$, we will adopt a new coordinate system in $N_{\epsilon}(W)$ (from the proof of Lemma (7.3) so that small translations of $W$ are again stable curves. Let $W^{\epsilon}$ be a $\mathcal{C}^{2}$ extension of $W$ of length $\epsilon$ at each end. Note that if $P^{+}(Z)$ and $P^{+}(Y)$ lie in the same homogeneity strip $\mathbb{H}_{k}$, then $d\left(P^{+}(Z), P^{+}(Y)\right) \leq C k^{2} d(Z, Y)$, since distances along stable curves are contracted by a factor of order 1 under $P^{+}$, while distances along unstable curves are expanded by a factor proportional to $k^{2}$. Since the maximum length of map-stable and -unstable curves in $\mathbb{H}_{k}$ is at most $k^{-3}$, this implies that

$$
d\left(P^{+}(Z), P^{+}(Y)\right) \leq B^{\prime} d(Z, Y)^{3 / 5}
$$

for some $B^{\prime}>0$ depending only on the maximum curvature and $\tau_{\max }$. Thus, $P^{+}\left(N_{\epsilon}(W)\right)$ lies in a $B^{\prime} \epsilon^{3 / 5}$-neighborhood of $P^{+}(W)$. Since we have assumed the homogeneity strip containing $P^{+}(W)$ has width at least $2 A \epsilon^{3 / 5}$, we choose $A$ large enough compared to $B^{\prime}$ that $P^{+}\left(N_{\epsilon}(W)\right)$ lies in the same homogeneity strip $\mathbb{H}_{k}$ as $W$; indeed, our assumptions imply $C k^{-3} \geq 2 A \epsilon^{3 / 5}$, so 
that the width of the stable cone in this neighborhood of $W$ is at least $\cos \varphi\left(P^{+}\right) \geq A^{\prime} \epsilon^{2 / 5}$, for some constant $A^{\prime}$, increasing with $A$.

Now using Remark6.1 and proceeding like in the decomposition in the proof of Lemma 2.11, we may define a smooth foliation of $N_{\epsilon}(W)$ by admissible stable curves with the following properties: (i) $W^{\epsilon}$ belongs to the foliation; (ii) the foliation is trivial in the flow direction, i.e., $W^{\epsilon}$ lies in a smooth surface formed by such stable curves, which is then flown a distance proportional to $\pm \epsilon$ to define the foliation of $N_{\epsilon}(W)$. It may be that this flow surface reaches $\partial \Omega_{0}$. In this case, we simply stop the foliation at $\partial \Omega_{0}$ so that it does not contain any collision points.

Fixing a transverse curve $\gamma_{0}$ in the surface containing $W^{\epsilon}$, we define local coordinates $(s, u, t)$, associating $W^{\epsilon}$ with the first coordinate, $\{(s, 0,0): s \in[-\epsilon-|W| / 2,|W| / 2+\epsilon]\}$, parametrized by arclength, $\gamma_{0}$ with the second coordinate, and the flow direction with the third. Due to the smoothness of the foliation, the Jacobian $J_{0}$ of the change of variables $(x, y, \omega) \mapsto(s, u, t)$ is uniformly $\mathcal{C}^{1}$. Also, it may be that in this coordinate system near $\partial \Omega_{0}$, some coordinate choices lie inside one of the scatterers. This is immaterial to the integral we must estimate since $f$ is taken to be simply 0 inside such scatterers.

We are now ready to proceed with the required estimate. We have, by Fubini,

$$
\begin{array}{r}
\int_{W}\left(\mathbb{M}_{\epsilon}(f)-f\right) \psi d m_{W}=\int_{W} \int_{\mathbb{R}^{3}} \eta_{\epsilon}(Z-Y)(f(Y)-f(Z)) d m(Y) \psi(Z) d m_{W}(Z) \\
=\int_{\mathbb{R}^{3}} \eta_{\epsilon}(Y) \int_{W}(f(Z-Y)-f(Z)) \psi(Z) J_{0}(Z-Y) d m_{W}(Z) d m(Y) \\
\leq\left|\eta_{\epsilon}\right|_{L^{1}} \sup _{Y \in N_{\epsilon}(0)}\left|\int_{W}(f(Z-Y)-f(Z)) \psi(Z) J_{0}(Z-Y) d m_{W}(Z)\right|,
\end{array}
$$

using that $\left|\eta_{\epsilon}\right|_{L^{1}}=1$. We next want to apply the definition of the unstable norm. For $Y \in N_{\varepsilon}(0)$, let $W_{Y}$ denote the stable curve corresponding to $W-Y$ in the adapted coordinates $(s, u, t)$ and lying in the interior of $\Omega_{0}$. $W_{Y}$ is necessarily an admissible stable curve by our choice of adapted coordinates. Let $\mathbf{h}_{Y}$ denote the translation map from $W_{Y}$ to $W$. Then,

$$
\int_{W} f(Z-Y) \psi(Z) J_{0}(Z-Y) d m_{W}=\int_{W_{Y}} f \psi \circ \mathbf{h}_{Y} J_{0} d m_{W_{Y}} .
$$

Note that $J \mathbf{h}_{Y}=1$. In light of (7.11), the following sublemma is the main estimate in the proof of Lemma 7.4

Sublemma 7.6. There exists $C>0$ such that for each $Y \in N_{\epsilon}(W)$,

$$
\left|\int_{W_{Y}} f \psi \circ \mathbf{h}_{Y} J_{0} d m_{W_{Y}}-\int_{W} f \psi J_{0} \circ \mathbf{h}_{Y}^{-1} d m_{W}\right| \leq C \epsilon^{r_{0}}\|f\|_{\mathcal{B}},
$$

where $r_{0}=\min \{\gamma, 1 /(2 q)\}$.

Proof of Sublemma 7.6. Notice that not every $W_{Y}$ has $d_{\mathcal{W}^{s}}\left(W_{Y}, W\right)<\infty$. To remedy this, let $\tau^{ \pm}(W)=\min _{W}\left\{\tau(\cdot), \tau_{-1}(\cdot)\right\}$ and define the local surface $W^{0}=\left\{\Phi_{t}(W):|t| \leq \tau^{ \pm}(W)\right\}$ obtained by flowing $W$. By arguments similar to those used to ensure (ii) in the proof of Lemma 3.10, for each $Y$ we may choose a stable curve $V_{Y}=\Phi_{t_{Y}}(W) \subset W^{0}$ such that $d_{\mathcal{W}}\left(V_{Y}, W_{Y}\right)<\sqrt{\epsilon}$. Indeed, there exists a constant $C_{\kappa}>0$, depending on the maximum curvature of stable and unstable curves, such that

$$
\left|t_{Y}\right| \leq C_{\kappa} \epsilon \text {, for all } Y \in N_{\varepsilon}(W) .
$$

It may be that $\Phi_{t_{Y}}(W)$ is in the midst of a collision. But in this case, we let $V_{Y}$ denote only that portion of $\Phi_{t_{Y}}(W)$ that has not undergone a collision during time $t \in\left[0, t_{Y}\right]$. The mismatch between $W$ and $V_{Y}$ is then of length at most $C \sqrt{\epsilon}$ and can be estimated as in (17.14) below. 
Now we estimate,

$$
\begin{aligned}
\int_{W_{Y}} f \psi \circ \mathbf{h}_{Y} & J_{0} d m_{W_{Y}}-\int_{W} f \psi J_{0} \circ \mathbf{h}_{Y}^{-1} d m_{W} \\
= & \int_{W_{Y}} f \psi \circ \mathbf{h}_{Y} J_{0} d m_{W_{Y}}-\int_{V_{Y}} f\left(\psi J_{0} \circ \mathbf{h}_{Y}^{-1}\right) \circ \Phi_{-t_{Y}} J_{V_{Y}} \Phi_{-t_{Y}} d m_{V_{Y}} \\
& +\int_{V_{Y}} f\left(\psi J_{0} \circ \mathbf{h}_{Y}^{-1}\right) \circ \Phi_{-t_{Y}} J_{V_{Y}} \Phi_{-t_{Y}} d m_{V_{Y}}-\int_{W} f \psi J_{0} \circ \mathbf{h}_{Y}^{-1} d m_{W} .
\end{aligned}
$$

We begin with the first difference above. Denote by $S_{W_{Y}}$ the natural map from the $r$-interval $I_{W_{Y}}$ (on the scatterer) to $W_{Y}$ as defined at the end of Subsection 2.2, and let $S_{V_{Y}}$ denote the analogous map for $V_{Y}$. Set $I_{Y}$ to be the common $r$-interval on which $P^{+}\left(W_{Y}\right)$ and $P^{+}\left(V_{Y}\right)$ are defined, and denote the corresponding matched subcurves by $W_{Y}^{\prime}$ and $V_{Y}^{\prime}$, as defined in Section 4.2. The unmatched (at most) two ends may be estimated separately, using the strong stable norm since these unmatched pieces have length at most $\sqrt{\epsilon}$ :

$$
\begin{aligned}
\int_{W_{Y} \backslash W_{Y}^{\prime}} f \psi \circ \mathbf{h}_{Y} J_{0} d m_{W_{Y}} & \leq\left|W_{Y} \backslash W_{Y}^{\prime}\right|^{1 / q}\|f\|_{s}\left|J_{0}\right|_{\mathcal{C}^{\beta}\left(W_{Y}\right)}\left|\psi \circ \mathbf{h}_{Y}\right|_{\mathcal{C}^{\beta}\left(W_{Y}\right)} \\
& \leq C \epsilon^{1 /(2 q)}\|f\|_{s},
\end{aligned}
$$

where we have used the smoothness of both $J_{0}$ and $\mathbf{h}_{Y}$. A similar estimate holds for the integral over $V_{Y} \backslash V_{Y}^{\prime}$.

On $I_{Y}$, define $\phi=\left(\psi \circ \mathbf{h}_{Y} \cdot J_{0}\right) \circ S_{W_{Y}} \circ S_{V_{Y}}^{-1}$, so that $d\left(\psi \circ \mathbf{h}_{Y} \cdot J_{0}, \phi\right)=0$. Since the derivatives of $S_{W_{Y}}$ and $S_{V_{Y}}^{-1}$ are uniformly bounded by Lemma 3.4, we get $|\phi|_{\mathcal{C}^{\alpha}\left(V_{Y}^{\prime}\right)} \leq C|\psi|_{\mathcal{C}^{\alpha}\left(W_{Y}^{\prime}\right)} \leq C^{\prime}$. Now we estimate the first difference in the matched part of equation (7.13),

$$
\begin{aligned}
& \left|\int_{W_{Y}^{\prime}} f \psi \circ \mathbf{h}_{Y} J_{0} d m_{W}-\int_{V_{Y}^{\prime}} f\left(\psi J_{0} \circ \mathbf{h}_{Y}^{-1}\right) \circ \Phi_{-t_{Y}} J_{V_{Y}} \Phi_{-t_{Y}} d m_{V_{Y}}\right| \\
& \leq\left|\int_{W_{Y}^{\prime}} f \psi \circ \mathbf{h}_{Y} J_{0} d m_{W}-\int_{V_{Y}^{\prime}} f \phi d m_{W}\right| \\
& \quad+\left|\int_{V_{Y}^{\prime}} f \phi d m_{W}-\int_{V_{Y}^{\prime}} f\left(\psi J_{0} \circ \mathbf{h}_{Y}^{-1}\right) \circ \Phi_{-t_{Y}} J_{V_{Y}} \Phi_{-t_{Y}} d m_{V_{Y}}\right| \\
& \leq C \epsilon^{\gamma}\|f\|_{u}+\|f\|_{s}\left|\phi-\left(\psi J_{0} \circ \mathbf{h}_{Y}^{-1}\right) \circ \Phi_{-t_{Y}} J_{V_{Y}} \Phi_{-t_{Y}}\right|_{\mathcal{C}^{\beta}\left(V_{Y}^{\prime}\right)} .
\end{aligned}
$$

where we used that $d_{\mathcal{W}^{s}}\left(P^{+}\left(W_{Y}^{\prime}\right), P^{+}\left(V_{Y}^{\prime}\right)\right)<\epsilon$ (recall we have removed the endpoint discrepancy). We proceed to estimate the norm of the test function in the second term. Since each term in the difference is bounded, we may estimate each difference separately. For $Z \in V_{Y}^{\prime}$, we have

$$
\left|\psi \circ \mathbf{h}_{Y} \circ S_{W_{Y}} \circ S_{V_{Y}}^{-1}(Z)-\psi \circ \Phi_{-t_{Y}}(Z)\right| \leq|\psi|_{\mathcal{C}^{\alpha}(W)} d\left(\mathbf{h}_{Y} \circ S_{W_{Y}} \circ S_{V_{Y}}^{-1}(Z), \Phi_{-t_{Y}}(Z)\right)^{\alpha} .
$$

Using again $d_{\mathcal{W}^{s}}\left(P^{+}\left(W_{Y}^{\prime}\right), P^{+}\left(V_{Y}^{\prime}\right)\right)<\epsilon$, we have, $d\left(S_{W_{Y}} \circ S_{V_{Y}}^{-1}(Z), Z\right) \leq C \epsilon$. Moreover, $\mathbf{h}_{Y}$ is simply translation by $Y$, and $|Y| \leq C \epsilon$ while $\left|t_{Y}\right| \leq C_{\kappa} \epsilon$ by (7.12). Then using the triangle inequality twice gives $d\left(\mathbf{h}_{Y} \circ S_{W_{Y}} \circ S_{V_{Y}}^{-1}(Z), \Phi_{-t_{Y}}(Z)\right) \leq C \epsilon$. Thus,

$$
\left|\psi \circ \mathbf{h}_{Y} \circ S_{W_{Y}} \circ S_{V_{Y}}^{-1}(Z)-\psi \circ \Phi_{-t_{Y}}(Z)\right| \leq C \epsilon^{\alpha},
$$

for each $Z \in V_{Y}^{\prime}$. For brevity, set $\psi_{1}=\psi \circ \mathbf{h}_{Y} \circ S_{W_{Y}} \circ S_{V_{Y}}^{-1}$ and $\psi_{2}=\psi \circ \Phi_{-t_{Y}}$. Now given $Z, Z^{\prime} \in V_{Y}^{\prime}$, we estimate on the one hand using the above,

$$
\left|\psi_{1}(Z)-\psi_{2}(Z)-\psi_{1}\left(Z^{\prime}\right)+\psi_{2}\left(Z^{\prime}\right)\right| d\left(Z, Z^{\prime}\right)^{-\beta} \leq 2 C \epsilon^{\alpha} d\left(Z, Z^{\prime}\right)^{-\beta},
$$


while on the other, using the Hölder continuity of $\psi_{1}$ and $\psi_{2}$ separately (since $\mathbf{h}_{Y}, S_{W_{Y}}, S_{V_{Y}}^{-1}$ and $\Phi_{-t_{Y}}$ are all smooth functions),

$$
\left|\psi_{1}(Z)-\psi_{2}(Z)-\psi_{1}\left(Z^{\prime}\right)+\psi_{2}\left(Z^{\prime}\right)\right| d\left(Z, Z^{\prime}\right)^{-\beta} \leq 2 C|\psi|_{\mathcal{C}^{\alpha}(W)} d\left(Z, Z^{\prime}\right)^{\alpha-\beta} .
$$

The Hölder constant is bounded by the minimum of the two estimates, which is maximized when the two are equal, i.e., when $d\left(Z, Z^{\prime}\right)=\epsilon$. Thus,

$$
\left|\psi_{1}-\psi_{2}\right|_{\mathcal{C}^{\beta}\left(V_{Y}^{\prime}\right)} \leq C \epsilon^{\alpha-\beta} .
$$

Next, we must estimate,

$$
\left|J_{0} \circ S_{W_{Y}} \circ S_{V_{Y}}^{-1}-J_{0} \circ \mathbf{h}_{Y}^{-1} \circ \Phi_{-t_{Y}}\right|_{\mathcal{C}^{\beta}\left(V_{Y}^{\prime}\right)} .
$$

But note that setting $\tilde{\psi}=J_{0} \circ \mathbf{h}_{Y}^{-1}$, this difference has precisely the same form as the difference just estimated concerning $\psi$. Since $J_{0}$ and $\mathbf{h}_{Y}^{-1}$ are both smooth functions, this estimate has the same bound as (7.16).

Finally, we must estimate $\left|1-J_{V_{Y}} \Phi_{-t_{Y}}\right|_{\mathcal{C}^{\beta}\left(V_{Y}^{\prime}\right)}$. Using the linearity of the flow between collisions and again (7.12), we have $\left|1-J_{V_{Y}} \Phi_{-t_{Y}}(Z)\right| \leq C \epsilon$ for all $Z \in V_{Y}^{\prime}$. Then since $J_{V_{Y}} \Phi_{-t_{Y}}$ is uniformly $\mathcal{C}^{1}$ on $V_{Y}$ due to the fact that $V_{Y}$ has uniformly bounded curvature, we may use the same technique as that above (7.16) to estimate $\left|1-J_{V_{Y}} \Phi_{-t_{Y}}\right|_{\mathcal{C}^{\beta}\left(V_{Y}^{\prime}\right)} \leq C \epsilon^{1-\beta}$.

Using these estimates on the test functions in (17.15) together with (7.14) yields the following estimate on the first difference in (7.13),

$$
\begin{aligned}
& \left|\int_{W_{Y}} f \psi \circ \mathbf{h}_{Y} J_{0} d m_{W_{Y}}-\int_{V_{Y}} f\left(\psi J_{0} \circ \mathbf{h}_{Y}^{-1}\right) \circ \Phi_{-t_{Y}} J_{V_{Y}} \Phi_{-t_{Y}} d m_{V_{Y}}\right| \\
& \leq C \epsilon^{1 /(2 q)}\|f\|_{s}+C \epsilon^{\gamma}\|f\|_{u}+C \epsilon^{\alpha-\beta}\|f\|_{s} .
\end{aligned}
$$

We proceed to estimate the second difference in (7.13). Using (4.27) and following,

$$
\begin{aligned}
\int_{\Phi_{t_{Y}}(W)} f(\psi & \left.J_{0} \circ \mathbf{h}_{Y}^{-1}\right) \circ \Phi_{-t_{Y}} J_{V_{Y}} \Phi_{-t_{Y}} d m_{V_{Y}}-\int_{W} f \psi J_{0} \circ \mathbf{h}_{Y}^{-1} d m_{W} \\
& =\int_{0}^{t_{Y}} \partial_{t} \int_{\Phi_{t}(W)} f\left(\psi J_{0} \circ \mathbf{h}_{Y}^{-1}\right) \circ \Phi_{-t} J_{\Phi_{t}(W)} \Phi_{-t} d m_{\Phi_{t}(W)} d t \\
& =\int_{0}^{t_{Y}} \partial_{t} \int_{W} f \circ \Phi_{t} \cdot \psi \cdot J_{0} \circ \mathbf{h}_{Y}^{-1} d m_{W} d t \\
& =\left.\int_{0}^{t_{Y}} \int_{W} \partial_{r}\left(f \circ \Phi_{r}\right)\right|_{r=0} \circ \Phi_{t} \cdot \psi \cdot J_{0} \circ \mathbf{h}_{Y}^{-1} d m_{W} d t \\
& =\left.\int_{0}^{t_{Y}} \int_{\Phi_{t}(W)} \partial_{r}\left(f \circ \Phi_{r}\right)\right|_{r=0}\left(\psi J_{0} \circ \mathbf{h}_{Y}^{-1}\right) \circ \Phi_{-t} \cdot J_{\Phi_{t}(W)} \Phi_{-t} d m_{\Phi_{t}(W)} d t \\
& \leq \int_{0}^{t_{Y}}\|f\|_{0}\left|\left(\psi J_{0} \circ \mathbf{h}_{Y}^{-1}\right) \circ \Phi_{-t} \cdot J_{\Phi_{t}(W)} \Phi_{-t}\right|_{\mathcal{C}^{\alpha}\left(\Phi_{t}(W)\right)} d t .
\end{aligned}
$$

On each stable curve $\Phi_{t}(W)$ the norm of the test function is bounded by a uniform constant since the flow is linear in $t$ between collisions, and both $\mathbf{h}_{Y}^{-1}$ and $J_{0}$ are $\mathcal{C}^{1}$ functions. Using these estimates and $\left|t_{Y}\right| \leq C_{\kappa} \epsilon$, we obtain the following estimate for the second difference in (7.13),

$$
\left|\int_{\Phi_{t_{Y}}(W)} f\left(\psi J_{0} \circ \mathbf{h}_{Y}^{-1}\right) \circ \Phi_{-t_{Y}} J_{V_{Y}} \Phi_{-t_{Y}} d m_{V_{Y}}-\int_{W} f \psi J_{0} \circ \mathbf{h}_{Y}^{-1} d m_{W}\right| \leq C \epsilon\|f\|_{0} .
$$

Putting this together with (7.17) and (7.13) completes the proof of Sublemma 7.6 since $\gamma \leq \alpha-\beta$ by definition of the norms. 
The sublemma being proved, we may return to the proof of Lemma 7.4; Sublemma 7.6 and (17.11) provide the required estimate under the assumption that $P^{+}(W)$ does not lie within a distance $A \epsilon^{3 / 5}$ of the boundary of a homogeneity strip.

Now suppose that $P^{+}(W)$ lies within a distance of $A \epsilon^{3 / 5}$ of the boundary of a homogeneity strip $\mathbb{H}_{k_{1}}$. Let $\bar{W}$ denote the one connected component of $W$ which does not project to within a distance of $A \epsilon^{3 / 5}$ of the boundary of $\mathbb{H}_{k_{1}}$ (note that this may be empty, for example in homogeneity strips of high index). We may perform the estimate on $\bar{W}$ precisely as in Sublemma 7.6.

By construction, $\bar{W} \backslash W$ consists of at most two components of length at most $A \epsilon^{3 / 5}$. Call one of them $W_{1}$. Although now $N_{\varepsilon}\left(W_{1}\right)$ may cross countably many homogeneity regions, we proceed using (7.9) and the decomposition of Lebesgue measure from the proof of Lemma 7.3. Now,

$$
\left|\int_{W_{1}} \psi(Z) d m_{W} \sum_{k, i} \int_{B_{i}^{0-}} f(Y) \eta_{\epsilon}(Z-Y) d m(Y)\right| \leq \sum_{k, i}\left|W_{1}\right| C \epsilon^{-2-\beta+1 / q} k^{-5}\|f\|_{s} .
$$

As in the proof of Lemma 7.3, the sum over $k$ yields a factor proportional to $\epsilon$. Finally, $\left|W_{1}\right| \leq$ $A \epsilon^{3 / 5}$, completing the proof of the lemma.

Proof of Lemma 7.5. Let $f \in \mathcal{C}^{1}\left(\Omega_{0}\right)$ and define $\mathbb{M}_{\epsilon}^{\sim} f=\mathbb{M}_{\epsilon}\left(f \cdot 1_{\Omega_{0} \backslash \partial_{\epsilon} \Omega_{0}}\right)$, where $\partial_{\epsilon} \Omega_{0}$ denotes the $\epsilon$-neighborhood of $\partial \Omega_{0}$ in $\Omega_{0}$. Note that $\mathbb{M}_{\epsilon}^{\sim} f \equiv 0$ on $\partial \Omega_{0}$, so that $\mathbb{M}_{\epsilon}^{\sim} f \in \mathcal{C}^{2}\left(\Omega_{0}\right) \cap \mathcal{C}_{\sim}^{0}$. We will show that $\mathbb{M}_{\epsilon}^{\sim} f$ is a good approximation of $f$ in $\mathcal{B}$. Note that $\nabla \mathbb{M}_{\epsilon}^{\sim} f=\mathbb{M}_{\epsilon}^{\sim}(\nabla f)$ so $\left|\mathbb{M}_{\epsilon}^{\sim} f\right|_{\mathcal{C}^{1}\left(\Omega_{0}\right)} \leq|f|_{\mathcal{C}^{1}\left(\Omega_{0}\right)}$. By the proof of Lemma 3.9, $\left\|\mathbb{M}_{\epsilon}^{\sim} f\right\|_{\mathcal{B}} \leq C|f|_{\mathcal{C}^{1}\left(\Omega_{0}\right)}$ for all $\epsilon>0$.

Let $W \in \mathcal{W}^{s}, \psi \in \mathcal{C}^{\beta}(W)$ with $|\psi|_{\mathcal{C}^{\beta}(W)} \leq|W|^{-1 / q}$. Assume for the moment that $W \cap \partial_{2 \epsilon} \Omega_{0}=$ $\emptyset$. Then using (17.3) and the fact that $\int \eta_{\epsilon}=1$,

$$
\begin{aligned}
& \int_{W}\left(f-\mathbb{M}_{\epsilon}^{\sim} f\right) \psi d m_{W}=\int_{W} \psi(Z) \int_{\mathbb{R}^{3}} \eta_{\epsilon}(Z-Y)(f(Z)-f(Y)) d m(Y) d m_{W}(Z) \\
& \leq|f|_{\mathcal{C}^{1}\left(\Omega_{0}\right)}|W|^{-1 / q} \int_{W} \int_{\mathbb{R}^{3}} \eta_{\epsilon}(Z-Y)|Z-Y| d m(Y) d m_{W}(Z) \leq \epsilon|f|_{\mathcal{C}^{1}\left(\Omega_{0}\right)},
\end{aligned}
$$

where in the last estimate, we have used that $q>1$ and $\eta_{\epsilon}(Z-Y)=0$ if $|Z-Y|>\epsilon$.

Next, if $W \cap \partial_{2 \varepsilon} \Omega_{0} \neq \emptyset$, then we subdivide $W$ into at most two components $W^{\prime}=W \cap \partial_{2 \varepsilon} \Omega_{0}$ and at most one component $W \backslash W^{\prime}$. On $W \backslash W^{\prime}$, the above estimate holds. On $W^{\prime}$, we use the fact that $\left|W^{\prime}\right| \leq C \epsilon^{1 / 2}$ (see, for example, Lemma 8.2) to estimate,

$$
\int_{W^{\prime}}\left(f-\mathbb{M}_{\epsilon}^{\sim} f\right) \psi d m_{W} \leq\left|W^{\prime}\right|^{1-1 / q} 2|f|_{\mathcal{C}^{0}\left(\Omega_{0}\right)} \leq C \epsilon^{\frac{1}{2}\left(1-\frac{1}{q}\right)}|f|_{\mathcal{C}^{0}\left(\Omega_{0}\right)} .
$$

Putting these two estimates together, we have

$$
\left\|f-\mathbb{M}_{\epsilon}^{\sim} f\right\|_{s} \leq C \epsilon^{\frac{1}{2}\left(1-\frac{1}{q}\right)}|f|_{\mathcal{C}^{1}(0)} .
$$

Next, we estimate the strong unstable norm. For $\varepsilon>0$, let $W_{1}, W_{2} \in \mathcal{W}^{s}$ with $d_{\mathcal{W}^{s}}\left(W_{1}, W_{2}\right)<$ $\varepsilon$. Let $\psi_{i} \in \mathcal{C}^{\alpha}\left(W_{i}\right)$ with $\left|\psi_{i}\right|_{\mathcal{C}^{\alpha}\left(W_{i}\right)} \leq 1$ and $d\left(\psi_{1}, \psi_{2}\right)=0$.

Let $s>0$ be a small number to be chosen below. First assume that $\epsilon^{\frac{1}{2}-s}<\varepsilon^{\gamma}$. Then using the estimates for the strong stable norm on each curve separately, we have,

$$
\varepsilon^{-\gamma}\left|\int_{W_{1}}\left(f-\mathbb{M}_{\epsilon}^{\sim} f\right) \psi_{1} d m_{W_{1}}-\int_{W_{2}}\left(f-\mathbb{M}_{\epsilon}^{\sim} f\right) \psi_{2} d m_{W_{2}}\right| \leq C \varepsilon^{-\gamma} \epsilon^{1 / 2}|f|_{\mathcal{C}^{1}\left(\Omega_{0}\right)} \leq C \epsilon^{s}|f|_{\mathcal{C}^{1}\left(\Omega_{0}\right)} .
$$

Notice that we do not have the exponent $1-1 / q$ in this estimate since the test functions used in the strong unstable norm are nicer than those used in the strong stable norm.

Now suppose $\epsilon^{\frac{1}{2}-s} \geq \varepsilon^{\gamma}$. Recalling the notation of Definition 2.10, we let

$$
W_{i}=\left\{S_{W_{i}}(r)=\Phi_{-t(r)} \circ G_{W_{i}}(r): r \in I_{i}\right\},
$$


where $G_{W_{i}}(r)=\left(r, \phi_{i}(r)\right)$ is the graph of $P^{+}\left(W_{i}\right)$ over the $r$-interval $I_{i}$. Denote by $U_{i} \subset W_{i}$ the maximal subcurve such that $P^{+}\left(U_{1}\right)$ and $P^{+}\left(U_{2}\right)$ are defined as graphs over the interval $I=I_{1} \cap I_{2}$. We have at most two pieces $V_{i}=W_{i} \backslash U_{i}$ and by definition of $d_{\mathcal{W}^{s}}(\cdot, \cdot)$ and Lemma 3.4 . we have $\left|V_{i}\right| \leq C\left|P^{+}\left(V_{i}\right)\right| \leq C \varepsilon$. Thus the estimate over the (at most two) unmatched pieces $V_{i}$ is,

$$
\varepsilon^{-\gamma}\left|\int_{V_{i}}\left(f-\mathbb{M}_{\epsilon}^{\sim} f\right) \psi_{1} d m_{W_{1}}\right| \leq C \varepsilon^{1-\gamma} 2|f|_{\mathcal{C}^{0}(\Omega)} \leq C \epsilon^{\left(\frac{1}{2}-s\right)\left(\frac{1}{\gamma}-1\right)} .
$$

It remains to estimate the norm of $f-\mathbb{M}_{\epsilon}^{\sim} f$ on matched pieces.

$$
\begin{gathered}
\int_{U_{1}}\left(f-\mathbb{M}_{\epsilon}^{\sim} f\right) \psi_{1} d m_{W_{1}}-\int_{U_{2}}\left(f-\mathbb{M}_{\epsilon}^{\sim} f\right) \psi_{2} d m_{W_{2}}=\int_{U_{1}} f \psi_{1} d m_{W_{1}} \\
-\int_{U_{2}} f \psi_{2} d m_{W_{2}}+\int_{U_{2}} \mathbb{M}_{\epsilon}^{\sim} f \psi_{2} d m_{W_{2}}-\int_{U_{1}} \mathbb{M}_{\epsilon}^{\sim} f \psi_{1} d m_{W_{1}} .
\end{gathered}
$$

To estimate the difference of integrals in $f$, we write, using the fact that $\psi_{1} \circ S_{W_{1}}=\psi_{2} \circ S_{W_{2}}$ by assumption,

$$
\begin{aligned}
\int_{U_{1}} f \psi_{1} d m_{W_{1}}- & \int_{U_{2}} f \psi_{2} d m_{W_{2}}=\int_{I}\left(f \circ S_{W_{1}} J S_{W_{1}}-f \circ S_{W_{2}} J S_{W_{2}}\right) \psi_{1} \circ S_{W_{1}} d r \\
& \leq C|I|\left|f \circ S_{W_{1}} \cdot J S_{W_{1}}-f \circ S_{W_{2}} \cdot J S_{W_{2}}\right|_{\mathcal{C}^{0}(I)} \\
& \leq C|I|\left(|f|_{\mathcal{C}^{1}\left(\Omega_{0}\right)}\left|S_{W_{1}}-S_{W_{2}}\right|_{\mathcal{C}^{0}(I)}+|f|_{\mathcal{C}^{0}\left(\Omega_{0}\right)}\left|J S_{W_{1}}-J S_{W_{2}}\right|_{\mathcal{C}^{0}(I)}\right) .
\end{aligned}
$$

For the first term above, since expansion in the stable direction from $P^{+}\left(W_{i}\right)$ to $W_{i}$ is of order 1 by Lemma 3.4, and since the unstable direction only contracts under this map, we have

$$
\left|S_{W_{1}}-S_{W_{2}}\right|_{\mathcal{C}^{0}(I)} \leq C\left|G_{W_{1}}-G_{W_{2}}\right|_{\mathcal{C}^{0}(I)} \leq C \varepsilon,
$$

by assumption on $W_{1}$ and $W_{2}$. For the second term, $\left|J S_{W_{1}}-J S_{W_{2}}\right|_{\mathcal{C}^{0}(I)} \leq C \varepsilon$, again using Lemma 3.4 and that $U_{1}$ and $U_{2}$ are $\mathcal{C}^{1}$-close as graphs over $I$ by definition of $d_{\mathcal{W}^{s}}\left(W_{1}, W_{2}\right)$. Thus,

$$
\varepsilon^{-\gamma}\left|\int_{U_{1}} f \psi_{1} d m_{W_{1}}-\int_{U_{2}} f \psi_{2} d m_{W_{2}}\right| \leq C \varepsilon^{1-\gamma}|f|_{\mathcal{C}^{1}\left(\Omega_{0}\right)} \leq C \epsilon^{\left(\frac{1}{2}-s\right)\left(\frac{1}{\gamma}-1\right)}|f|_{\mathcal{C}^{1}\left(\Omega_{0}\right)},
$$

completing the estimate on the first term of (7.20). The second term is estimated similarly, using the fact that $\left|\eta_{\epsilon}\right|_{\mathcal{C}^{1}\left(\Omega_{0}\right)} \leq C \epsilon^{-1}|f|_{\mathcal{C}^{0}\left(\Omega_{0}\right)}$

$$
\begin{aligned}
\varepsilon^{-\gamma}\left|\int_{U_{1}} \mathbb{M}_{\epsilon}^{\sim} f \psi_{1} d m_{W_{1}}-\int_{U_{2}} \mathbb{M}_{\epsilon}^{\sim} f \psi_{2} d m_{W_{2}}\right| & \leq C \varepsilon^{1-\gamma}\left|\mathbb{M}_{\epsilon}^{\sim} f\right|_{\mathcal{C}^{1}\left(\Omega_{0}\right)} \\
& \leq C \epsilon^{\left(\frac{1}{2}-s\right)\left(\frac{1}{\gamma}-1\right)-1}|f|_{\mathcal{C}^{0}\left(\Omega_{0}\right)} .
\end{aligned}
$$

Putting together these estimates on the terms in (7.20) with the estimate on unmatched pieces yields an exponent of $\epsilon$ at worst $\left(\frac{1}{2}-s\right)\left(\frac{1}{\gamma}-1\right)-1$. Since $\gamma<1 / 3$, we have $\frac{1}{\gamma}-1>2$, and so we may choose $s>0$ sufficiently small that the above exponent is positive.

Finally, we estimate the neutral norm. Fix $W \in \mathcal{W}^{s}$ and $\psi \in \mathcal{C}^{\alpha}(W)$ with $|\psi|_{\mathcal{C}^{\alpha}(W)} \leq 1$. Recall that $\hat{\eta}$ denotes the unit vector in the flow direction. Then, $\left.\partial_{t}\left(f \circ \Phi_{t}\right)\right|_{t=0}=\nabla f \cdot \hat{\eta}$ and also for $Z \in \Omega_{0} \backslash \partial_{2 \epsilon} \Omega_{0}$,

$$
\left.\partial_{t}\left(\left(\mathbb{M}_{\epsilon}^{\sim} f\right) \circ \Phi_{t}\right)\right|_{t=0}(Z)=\mathbb{M}_{\epsilon}^{\sim}(\nabla f \cdot \hat{\eta})(Z) .
$$

Now suppose $W \cap \partial_{2 \epsilon} \Omega_{0}=\emptyset$. Then,

$$
\left.\int_{W} \partial_{t}\left(f-\mathbb{M}_{\epsilon}^{\sim} f\right) \circ \Phi_{t}\right|_{t=0} \psi d m_{W}=\int_{W} \psi(Z) \int_{\mathbb{R}^{3}} \eta_{\epsilon}(Z-Y)(\nabla f \cdot \hat{\eta}(Z)-\nabla f \cdot \hat{\eta}(Y)) .
$$


Now since $\nabla f$ is uniformly continuous on $\Omega_{0}$, there exists a function $\rho(\epsilon)$ with $\rho(\epsilon) \downarrow 0$ as $\epsilon \downarrow 0$, such that $|\nabla f(Z)-\nabla f(Y)| \leq \rho(\epsilon)$ whenever $|Z-Y|<\epsilon$. Since the flow direction $\hat{\eta}$ also changes smoothly on $\Omega_{0}$, we have

$$
\left|\int_{W} \partial_{t}\left(f-\mathbb{M}_{\epsilon}^{\sim} f\right) \circ \Phi_{t}\right|_{t=0} \psi d m_{W} \mid \leq C \rho(\epsilon) .
$$

If, on the other hand, $W \cap \partial_{2 \epsilon} \Omega_{0} \neq \emptyset$, then using the same decomposition of $W$ as in the proof the strong stable norm estimate, we have

$$
\left.\int_{W^{\prime}} \partial_{t}\left(f-\mathbb{M}_{\epsilon}^{\sim} f\right) \circ \Phi_{t}\right|_{t=0} \psi d m_{W} \leq 2\left|W^{\prime}\right||\nabla f|_{\mathcal{C}^{0}\left(\Omega_{0}\right)} \leq C \epsilon^{1 / 2}|f|_{\mathcal{C}^{1}\left(\Omega_{0}\right)} .
$$

Since $f$ can be approximated by functions in $\mathcal{C}^{2}\left(\Omega_{0}\right) \cap \mathcal{C}_{\sim}^{0}$ with uniformly bounded $\|\cdot\|_{\mathcal{B}}$-norms, it follows that $f \in \mathcal{B}$.

\section{The Dolgopyat Cancellation estimate (Lemma 8.10)}

Recall the hyperbolicity exponent $\Lambda=\Lambda_{0}^{1 / \tau_{\max }}>1$ from (1.2).

Lemma 8.1 (Dolgopyat bound). There exists $C_{\#}>0$, and, for any $0<\alpha \leq 1 / 3$, there exist $C_{D o}>0$ and $\gamma_{D o}>0$ so that for any homogeneous curve $W \in \mathcal{W}^{s}$ of length $|W|=m_{W}(W) \leq 1$ and any $f \in \mathcal{C}^{1}\left(\mathbb{T}^{3}\right)$

$$
\sup _{\substack{\psi \in \mathcal{C}^{\alpha}(W) \\|\psi|_{\mathcal{C}^{\alpha}(W)} \leq 1}} \int_{W} \psi \mathcal{R}(a+i b)^{2 m}(f) d m_{W} \leq \frac{C_{\#}}{a^{2 m} b^{\gamma_{D o}}}\left(|f|_{L^{\infty}\left(\Omega_{0}\right)}+\left(1+a^{-1} \ln \Lambda\right)^{-m}|f|_{H_{\infty}^{1}\left(\Omega_{0}\right)}\right),
$$

$$
\forall 1<a<2, \quad \forall b>1, \quad \forall m \geq C_{D o} \ln b .
$$

Since $\overline{\mathcal{R}(a+i b)}=\mathcal{R}(a-i b)$ the obvious counterpart of the above lemma holds for $b<0$.

Note that we do not require the $\delta$-averaging operator used in [L2, BL]: Since the weak norm in the right-hand side of the Lasota-Yorke estimates already involves averaging over stable curves, we can use the weak norm directly.

The rest of the section contains the proof of Lemma 8.1 and consists of a direct, but lengthy and highly non trivial, computation. In the present section, $C_{\#}$ denotes a constant depending only on the billiard dynamics and not on $\gamma, 1 / q, \beta$, or $\alpha$.

First of all note that if $|W| \leq b^{-\gamma_{D o}}$, then the statement of the lemma is trivially true. We can thus assume that $|W| \geq b^{-\gamma_{D o}}$.

It is more convenient to work directly with the flow rather than with the Poincare sections, and we will consider time steps $\tau_{-} \ll \tau_{\min }$. The precise value of $\tau_{-}$will be chosen later in (8.9).

To compute the integral, it is helpful to localize it in space-time. To localize in time, consider a smooth function $\tilde{p}: \mathbb{R} \rightarrow \mathbb{R}$ such that $\operatorname{supp} \tilde{p} \subset(-1,1), \tilde{p}(s)=\tilde{p}(-s)$, and $\sum_{\ell \in \mathbb{Z}} \tilde{p}(t-\ell)=1$ for all $t \in \mathbb{R}$. For $f \in L^{\infty}\left(\Omega_{0}, \operatorname{vol}\right)$, we set $p(s)=\tilde{p}\left(\tau_{-}^{-1} s\right)$ and write

$$
\begin{aligned}
\mathcal{R}(z)^{m}(f) & =\sum_{\ell \in \mathbb{Z}} \int_{0}^{\infty} p\left(t-\ell \tau_{-}\right) \frac{t^{m-1}}{(m-1) !} e^{-z t} \mathcal{L}_{t} f d t \\
(8.1) \quad & =\sum_{\ell \in \mathbb{N}^{*}} \int_{-\tau_{-}}^{\tau_{-}} p(s) \frac{\left(s+\ell \tau_{-}\right)^{m-1}}{(m-1) !} e^{-z \ell \tau_{-}-z s} \mathcal{L}_{\ell \tau_{-}} \mathcal{L}_{s} f d s+\int_{0}^{\tau_{-}} p(s) \frac{s^{m-1}}{(m-1) !} e^{-z s} \mathcal{L}_{s} f d s .
\end{aligned}
$$

Next, setting for $\ell \geq 1$,

$$
p_{m, \ell, z}(s)=p(s) \frac{\left(s+\ell \tau_{-}\right)^{m-1}}{(m-1) !} e^{-z \ell \tau_{-}-z s}, \text { and } p_{m, 0, z}(s)=p(s) \frac{s^{m-1}}{(m-1) !} e^{-z s} \mathbf{I d}_{\{s \geq 0\}},
$$

\footnotetext{
${ }^{35}$ We do not assume here that $f$ is supported in $\Omega_{0}$, recalling (7.4).
} 
we fix $\psi \in \mathcal{C}^{\alpha}(W)$ with $|\psi|_{\mathcal{C}^{\alpha}(W)} \leq 1$ and write

$$
\int_{W} \psi \cdot\left(\mathcal{R}(z)^{m}(f)\right) d m_{W}=\sum_{\ell \in \mathbb{N}} \int_{-\tau_{-}}^{\tau_{-}} p_{m, \ell, z}(s) \int_{W} \psi \cdot \mathcal{L}_{\ell \tau_{-}} \mathcal{L}_{s} f d m_{W} d s .
$$

Changing variables, we rewrite $(\underline{8.3})$ as

$$
\begin{gathered}
\int_{W} \psi \cdot\left(\mathcal{R}(z)^{m}(f)\right) d m_{W}=\sum_{\ell \in \mathbb{N}} \int_{-\tau_{-}}^{\tau_{-}} p_{m, \ell, z}(s) \int_{\Phi_{-\ell \tau_{-}} W} J_{\ell \tau_{-}}^{s} \cdot \psi \circ \Phi_{\ell \tau_{-}} \cdot \mathcal{L}_{s} f d s \\
=\sum_{\ell \in \mathbb{N}} \sum_{W_{A} \in \widehat{\mathcal{G}}_{\ell \tau_{-}}(W)} \int_{-\tau_{-}}^{\tau_{-}} p_{m, \ell, z}(s) \int_{W_{A}} J_{\ell \tau_{-}}^{s} \cdot \psi \circ \Phi_{\ell \tau_{-}} \cdot \mathcal{L}_{s} f d m_{W_{A}} d s,
\end{gathered}
$$

where $J_{\ell \tau_{-}}^{s}=J_{W_{A}}^{s} \Phi_{\ell \tau_{-}}$is the (stable) Jacobian of the change of variable and $\widehat{\mathcal{G}}_{\ell \tau_{-}}(W):=$ $\left\{W_{A}\right\}_{A \in A_{\ell}}$ is the decomposition of $\Phi_{-\ell \tau_{-}} W$ specified in Definition 3.1. By Lemma 3.2. for each $A \in A_{\ell}$ there exists $t_{A} \in\left[0, \tau_{\text {min }}\right]$ such that $\Phi_{-t_{A}} W_{A}$ is a $\mathcal{C}^{2}$ curve with uniform $\mathcal{C}^{2}$ norm and $\Phi_{\ell \tau_{+}-t_{A}}$, restricted to $\Phi_{-t_{A}} W_{A}$ is a $\mathcal{C}^{2}$ map.

Lemma 3.5 implies that $C_{\#}^{-1} \frac{\left|\Phi_{\ell \tau_{-}} W_{A}\right|}{\left|W_{A}\right|} \leq J_{\ell \tau_{-}}^{s} \leq C_{\#} \frac{\left|\Phi_{\ell \tau_{-}} W_{A}\right|}{\left|W_{A}\right|}$, provided $C_{\#}$ is chosen large enough. Note that $\sum_{A} \int_{W_{A}} J_{\ell \tau_{-}}^{s}=|W|$.

Next, we shall localize in space as well, introducing in (8.7) below a sequence of smooth partitions of unity parametrised by

$$
\theta \in(0,1) \text { and } \varepsilon \in\left(0, L_{0}\right)
$$

to be chosen later. First, we need some preparations: We shall exclude a neighborhood of $\partial \Omega_{0}$ since 36 the angle of the stable and unstable cones can change quickly near this boundary. We shall also exclude a neighborhood of the surfaces on which either the stable or unstable cones we have defined are discontinuous. Define

$$
S^{0-}=\left\{Z \in \Omega_{0}: \varphi\left(P^{+}(Z)\right)= \pm \pi / 2\right\} \quad \text { and } \quad S^{0+}=\left\{Z \in \Omega_{0}: \varphi\left(P^{-}(Z)\right)= \pm \pi / 2\right\},
$$

and their $\varepsilon$-neighborhoods, $S_{\varepsilon}^{0 \pm}=\left\{Z \in \Omega_{0}: d\left(Z, S^{0 \pm}\right)<\varepsilon\right\}$. Note that $S^{0-}$ is the flow of the singularity curve for the map $\mathcal{S}_{0}=\{ \pm \pi / 2\}$ backwards until its first collision, while $S^{0+}$ is the flow of $\mathcal{S}_{0}$ forwards until its next collision. Stable cones are discontinuous across the surface $S^{0-}$ while unstable cones are discontinuous across $S^{0+}$. Similarly, let $\partial_{\varepsilon}\left(\Omega_{0}\right)$ denote the $\varepsilon$-neighborhood of $\partial \Omega_{0}$ within $\Omega_{0}$. The following lemma shows that the curves in $\mathcal{W}^{s}$ have small intersections with such sets. We will then be able to discard such intersections and this will allow us to introduce special charts as in Remark 6.1, see Remark 8.3 below. This lemma has no analogue in [BL, where the situation was much easier.

Lemma 8.2. There exists $C_{\#}>0$ such that for any $\varepsilon \in\left[0, L_{0}\right]$ and any homogeneous $W \in \mathcal{W}^{s}$,

$$
\left|W \cap S_{\varepsilon}^{0-}\right| \leq C_{\#} \varepsilon^{3 / 5}, \quad\left|W \cap S_{\varepsilon}^{0+}\right| \leq C_{\#} \varepsilon, \quad\left|W \cap \partial_{\varepsilon} \Omega_{0}\right| \leq C_{\#} \varepsilon^{1 / 2} .
$$

Similar bounds hold for unstable curves with the estimates on $S_{\varepsilon}^{0-}$ and $S_{\varepsilon}^{0+}$ reversed.

Proof. Let $V=W \cap S_{\varepsilon}^{0-}$. The curve $P^{+}(W)$ is a homogeneous stable curve by assumption. Due to the uniform transversality of the map-stable cones with horizontal lines, $P^{+}(V)$ is uniformly transverse to $\mathcal{S}_{0}=\{\varphi= \pm \pi / 2\}$. Suppose $P^{+}(V)$ lies in a homogeneity strip of index $k$. The expansion in the stable cone from $P^{+}(V)$ to $V$ is of order 1, by Lemma 3.4. On the other hand, vectors in the unstable cone undergo an expansion of order $k^{2}$. Thus the angle between $V$ and $S^{0-}$ is no smaller than order $k^{-2}$, which means that $|V|$ is bounded by a uniform constant times $\varepsilon k^{2}$. But again using Lemma 3.4, we have the length of $|V|$ comparable to $\left|P^{+}(V)\right|$, which is at

\footnotetext{
36 In such a way in the following we will never need to consider a curve in the the midst of a collision.
} 
most $k^{-3}$. Since this holds for any $V^{\prime}$ in the same homogeneity strip, in particular it is true of a curve $V^{\prime}$ of length $k^{-3}$ (even if $V$ itself is shorter). Thus up to a uniform constant, we must have $k^{-3} \leq \varepsilon k^{2}$, which implies $k \geq \varepsilon^{-1 / 5}$. This means $\left|P^{+}(V)\right|$ and so $|V|$ is at most a uniform constant times $\varepsilon^{3 / 5}$.

The analysis is similar for $V=W \cap S_{\varepsilon}^{0+}$. Note that $S^{0+}$ is a surface which may divide $V$ into several components. Let $V_{1}$ denote a component of $V$ on one side of $S^{0+}$, so that $P^{-}\left(V_{1}\right)$ is contained in a single scatterer. Since $P^{-}\left(V_{1}\right)$ is a stable curve, even though it may not be homogeneous, it is uniformly transverse to $\mathcal{S}_{0}$. Since $S^{0+}$ is the surface defined by flowing $\mathcal{S}_{0}$ forward, $S^{0+}$ will remain uniformly transverse to $V_{1}$. Thus $\left|V_{1}\right| \leq C_{\#} \varepsilon$. Since the number of such components of $V$ is uniformly bounded by $\left\lfloor\frac{\tau_{\max }}{\tau_{\min }}\right\rfloor+1$ [CM, §5.10], the bound on $|V|$ follows.

Finally, let $V=W \cap \partial_{\varepsilon} \Omega_{0}$. Recall our global coordinates $(x, y, \omega)$ from Section 1.1. Although $V$ may not be uniformly transverse to $\partial \Omega$ (consider when $V$ is close to making a normal collision with a scatterer), the estimate will follow from the fact that its curvature is uniformly bounded away from the curvature of the scatterer.

Note that the (absolute value) of the curvature of a stable wavefront (the projection of a stable curve in the $(x, y)$-plane) just after a collision is given by $B^{+}=B^{-}+\frac{2 K}{\cos \varphi}$, where $B^{-}$is the curvature just before collision; between collisions, the curvature evolves according to $B_{t}=\frac{B_{0}^{+}}{B_{0}^{+}+t}$ [CM, §3.8]. Putting these together and using the definition of our stable cones for the flow, we see that the minimum curvature for any stable curve is $B_{\min }=\frac{2 \mathcal{K}_{\min }}{2 \mathcal{K}_{\min }+\tau_{\max }}$.

Now if $V$ is a component of a stable curve about to make a nearly normal collision in backward time, then the curvatures of $V$ and the scatterer are convex in opposition, both with minimum curvatures bounded away from 0 by our calculation above. Thus the length of the projection of $V$ in the $(x, y)$-plane is bounded by $C \varepsilon^{1 / 2}$ and since the slope of $|V|$ in the $(d \xi, d \omega)$-plane just before collision is bounded above by $2 / \tau_{\min }$, we have $|V| \leq C \varepsilon^{1 / 2}$ as well. If, on the other hand, $V$ has just made a nearly normal collision, then the curvature of its projection for a short time afterward is at least

$$
B^{+} \geq B_{\min }+\frac{\mathcal{K}}{\cos \varphi}
$$

while the curvature of the scatterer is $\mathcal{K}$. Thus the curvatures of the two projections are bounded away from one another, and so $|V| \leq C \varepsilon^{1 / 2}$ as before.

The last case to consider is if $V$ is close to a tangential collision. In this case, the projection of $V$ in the $(x, y)$-plane is uniformly transverse to the boundary of the scatterer; however, by the proof of Lemma 3.2, the length of $V$ in the $\omega$-coordinate undergoes an expansion of order $1 / \cos \varphi$ near such tangential collisions, and so again, $|V| \leq C \varepsilon^{1 / 2}$.

Let $c>2$ be a constant that will be chosen shortly and let $N_{\varepsilon^{\theta}}(Z)$ denote the neighborhood of $Z$ in $\Omega_{0}$ of size $\varepsilon^{\theta}$ in the standard $(x, y, \omega)$ coordinates. There exists $C_{\#}>0$ such that, for each $\varepsilon \in\left(0, L_{0}\right)$, there exists a $\mathcal{C}^{\infty}$ partition of unity of $\Omega_{0}$

$$
\left\{\phi_{\varepsilon, i}\right\}_{i=0}^{q(\varepsilon)}
$$

enjoying the following properties

(i) for each $i \in\{1, \ldots, q(\varepsilon)\}$, there exists $x_{i} \in \Omega_{0}$ such that $\phi_{\varepsilon, i}(z)=0$ for all $z \notin N_{\varepsilon^{\theta} / c}\left(x_{i}\right)$;

(ii) for each $\varepsilon, i$ we have $\left|\nabla \phi_{\varepsilon, i}\right|_{\infty} \leq C_{\#} \varepsilon^{-\theta}$;

(iii) $q(\varepsilon) \leq C_{\#} \varepsilon^{-3 \theta}$;

(iv) for each $\varepsilon>0$ we have $\operatorname{supp}\left(\phi_{\varepsilon, 0}\right) \subset \partial_{3 c \varepsilon^{\theta}} \Omega_{0} \cup S_{3 c \varepsilon^{\theta}}^{0-} \cup S_{3 c \varepsilon^{\theta}}^{0+}$, and, for every $i \geq 1$, $\operatorname{supp}\left(\phi_{\varepsilon, i}\right) \subset \Omega_{0} \backslash\left(\partial_{2 c \varepsilon^{\theta}} \Omega_{0} \cup S_{2 c \varepsilon^{\theta}}^{0-} \cup S_{2 c \varepsilon^{\theta}}^{0+}\right)$. 
Remark 8.3 ( $\mathcal{C}^{2}$ cone-compatible Darboux charts). Following Remark 6.1, we setup a similar family of local charts as announced in Remark 2.4: For any $Z \in \Omega_{0} \backslash\left(\partial_{2 c \varepsilon^{\theta}} \Omega_{0} \cup S_{2 c \varepsilon^{\theta}}^{0-} \cup S_{2 c \varepsilon^{\theta}}^{0+}\right)$, it follows from the proof of Lemma 8.2 that $\cos \varphi\left(P^{+}(Z)\right) \geq \varepsilon^{\theta / 2}$ for c sufficiently large. Similarly, if $Z^{\prime} \in \Omega_{0}$ satisfies $d\left(Z, Z^{\prime}\right)<c \varepsilon^{\theta}$, we have $\tau\left(Z^{\prime}\right) \geq c \varepsilon^{\theta}$ and $\cos \varphi\left(P^{+}\left(Z^{\prime}\right)\right) \geq \varepsilon^{\theta / 2}$. Thus it follows from (2.4) that the width of the stable cones at $Z$ and $Z^{\prime}$ has angle at least of order $\varepsilon^{\theta / 2}$, and the maximum and minimum slopes in $C^{s}(Z)$ and $C^{s}\left(Z^{\prime}\right)$ are uniformly bounded multiples of one another. Similar considerations hold for the unstable cones. Indeed, given the relation $k \leq \varepsilon^{-\theta / 5}$ and the fact that $d\left(P^{+}(Z), P^{+}\left(Z^{\prime}\right)\right)<C_{\#} \varepsilon^{\theta}$ by Lemma 3.4, we conclude that $P^{+}(Z)$ and $P^{+}\left(Z^{\prime}\right)$ must lie either in the same homogeneity strip or in adjacent homogeneity strips for small $\varepsilon$; specifically, $\varepsilon^{\theta} \leq C^{\prime} k^{-5}$, where $C^{\prime}$ depends only on $c$, the distortion of $P^{+}$given by Lemma 3.4] and the spacing of the homogeneity strips. Thus for each $i$, we may adopt local coordinates in a $c \varepsilon^{\theta}$ neighborhood of $x_{i},\left(x^{u}, x^{s}, x^{0}\right)$ as introduced in Remark 2.4 for which the contact form is in standard form and $x_{i}$ is the origin, and where $c$ is large enough so that $N_{\varepsilon^{\theta} / c}\left(x_{i}\right) \subset B_{\varepsilon^{\theta}}\left(x_{i}\right)$, where $B_{\varepsilon^{\theta}}\left(x_{i}\right)$ denotes the ball of radius $\varepsilon^{\theta}$ centered at $x_{i}$, in the sup norm of the $\left(x^{u}, x^{s}, x^{0}\right)$ coordinates. $B y$ the above discussion, the charts effecting this change of coordinates are uniformly $\mathcal{C}^{2}$.

We will refer to the two sides of the box $B_{\varepsilon^{\theta}}\left(x_{i}\right)$ comprising approximate weak stable manifolds as "stable sides," the two sides comprising approximate weak unstable manifolds as "unstable sides" and the two remaining sides as "flow sides."

Fix $c>2$ large enough and choose $\varepsilon$ small enough so that any manifold $W_{A}$ intersecting $B_{\varepsilon^{\theta}}\left(x_{i}\right)$ can intersect only (see [BL, Fig. 1]) the weak unstable sides of the boundary of $B_{c \varepsilon^{\theta}}\left(x_{i}\right)$. (This is possible since the $W_{A}$ belong to the stable cone and the cones vary continuously in $\Omega_{0} \backslash\left(\partial_{2 c \varepsilon^{\theta}} \Omega_{0} \cup S_{2 c \varepsilon^{\theta}}^{0-} \cup S_{2 c \varepsilon^{\theta}}^{0+}\right)$.) Define for $\ell \geq 0$,

$$
A_{\ell, 0}=D_{\ell, 0}=\left\{A \in A_{\ell}: W_{A} \cap\left(\partial_{2 c \varepsilon^{\theta}} \Omega_{0} \cup S_{2 c \varepsilon^{\theta}}^{0-} \cup S_{2 c \varepsilon^{\theta}}^{0+}\right) \neq \emptyset\right\}
$$

and for each $x_{i}, i \geq 1$, let

$$
\begin{gathered}
A_{\ell, i}=\left\{A \in A_{\ell}: W_{A} \cap B_{\varepsilon^{\theta}}\left(x_{i}\right) \neq \emptyset\right\}, \\
D_{\ell, i}=\left\{A \in A_{\ell, i}: \partial\left(W_{A} \cap B_{c \varepsilon^{\theta}}\left(x_{i}\right)\right) \not \subset \partial B_{c \varepsilon^{\theta}}\left(x_{i}\right)\right\}, \quad E_{\ell, i}=A_{\ell, i} \backslash D_{\ell, i} .
\end{gathered}
$$

The manifolds with index in $A_{\ell, i}$ are those which intersect the small box $B_{\varepsilon^{\theta}}\left(x_{i}\right)$, while $E_{\ell, i} \subset A_{\ell, i}$ consists of the indices of those manifolds which go completely across the big box $B_{c \varepsilon^{\theta}}\left(x_{i}\right)$. The remaining manifolds of $A_{\ell, i}$, with indexes in $D_{\ell, i}$, are called the discarded manifolds. We set $W_{A, i}=W_{A} \cap B_{c \varepsilon^{\theta}}\left(x_{i}\right)$ for $i \geq 1, W_{A, 0}=W_{A} \cap \partial_{3 c \varepsilon^{\theta}} \Omega_{0} \cup S_{3 c \varepsilon^{\theta}}^{0-} \cup S_{3 c \varepsilon^{\theta}}^{0+}$, and

$$
Z_{\ell, A, i}=\int_{W_{A, i}} J_{\ell \tau_{-}}^{s} d m_{W_{A, i}}, \quad i \geq 0
$$

It is now natural to choose

$$
\tau_{-}=c \varepsilon^{\theta} .
$$

We are going to worry about the small $\ell$ first. Let $a=\Re(z)$. Remembering (8.2), setting

$$
\ell_{0}:=\frac{m}{a e^{2} \tau_{-}}
$$

and using Stirling's formula, we have

$$
\begin{aligned}
& \left|\sum_{\ell \leq \ell_{0}} \int_{-\tau_{-}}^{\tau_{-}} p_{m, \ell, z}(s) \int_{W} \psi \cdot \mathcal{L}_{\ell \tau_{-}+s} f d m_{W} d s\right| \leq|f|_{\infty}|\psi|_{\infty}|W| \int_{0}^{\ell_{0} \tau_{-}} e^{-a s} \frac{s^{m-1}}{(m-1) !} d s \\
& \quad \leq C_{\#} \frac{|f|_{\infty}|\psi|_{\infty}}{(m-1) ! a^{m}} \int_{0}^{e^{-2} m} e^{-x} x^{m-1} d x \leq C_{\#} \frac{|f|_{\infty}|\psi|_{\infty}\left(e^{-2} m\right)^{m}}{m ! a^{m}} \leq C_{\#} \frac{|f|_{\infty}|\psi|_{\infty}}{a^{m} e^{m}},
\end{aligned}
$$


where we have made the substitution $x=a s$ in the second line. We impose that

$$
e^{-m} \leq \varepsilon^{\frac{\theta}{2}} .
$$

Our next step is to estimate the contribution of the discarded manifolds corresponding to indices in $D_{\ell, i}$ and $\ell \geq \ell_{0}$. For fixed $\ell$, we use the fact that the $B_{\varepsilon^{\theta}}\left(x_{i}\right)$ have a bounded number of overlaps as well as the fact that the number of components corresponding to a $D_{\ell, i}$ for each curve $W_{A} \in \mathcal{G}_{\ell \tau_{-}}(W)$ is uniformly bounded to estimate

$$
\begin{aligned}
\sum_{i \geq 0} \sum_{A \in D_{\ell, i}} Z_{\ell, A, i} & \leq C_{\#} \sum_{i \geq 0} \sum_{A \in D_{\ell, i}}\left|W_{A, i}\right|\left|J_{\ell \tau_{-}}^{s}\right|_{L^{\infty}\left(W_{A, i}\right)} \\
& \leq C_{\#} \varepsilon^{\theta / 2} \sum_{W_{A} \in \mathcal{G}_{\ell \tau_{-}}(W)}\left|J_{\ell \tau_{-}}^{s}\right|_{L^{\infty}\left(W_{A}\right) \leq C_{\#} \varepsilon^{\theta / 2},}
\end{aligned}
$$

where we have used Lemma 8.2 to bound the lengths of the discarded curves and Lemma 3.8 to bound the sum over the Jacobians.

Remark 8.4 (About Lemma 6.3 in [BL]). The statement of [BL, Lemma 6.3] is incorrect due to a missing sum over $\ell$ there (the error occurs in [BL, (E.6)] since $\Omega_{\beta, i}$ is two-dimensional while the neighborhood of $\widetilde{O}$ is three-dimensional). The argument of [BL, §6] can be fixed either by using Lemma 6.8 of $[\mathrm{BL}]$, or by providing a direct argument in the spirit of the one above.

Now using (8.13) and remembering (8.2), we have

$$
\begin{aligned}
& \left|\sum_{\ell \geq \ell_{0}} \sum_{i \geq 0} \sum_{A \in D_{\ell, i}} \int_{-\tau_{-}}^{\tau_{-}} p_{m, \ell, z}(s) \int_{W_{A, i}} \psi \circ \Phi_{\ell \tau_{-}} \cdot J_{\ell \tau_{-}}^{s} \cdot \phi_{\varepsilon, i} \cdot \mathcal{L}_{s} f\right| \\
& \leq C_{\#} \varepsilon^{\frac{\theta}{2}}|f|_{\infty}|\psi|_{\infty} \int_{\ell_{0} \tau_{-}}^{\infty} \frac{t^{m-1}}{(m-1) !} e^{-a t} d t \leq C_{\#} \varepsilon^{\frac{\theta}{2}} a^{-m}|f|_{\infty}|\psi|_{\infty} .
\end{aligned}
$$

We are left with the elements of $E_{\ell, i}$ for $\ell \geq \ell_{0}$. To study them, it is convenient to set

$$
W_{A}^{0}=\cup_{t \in\left[-\tau_{-}, \tau_{-}\right]} \Phi_{t} W_{A} \quad \forall A \in A_{\ell}, \quad W^{0}=\cup_{t \in\left[-\tau_{-}, \tau_{-}\right]} \Phi_{t} W .
$$

To continue, for each $x_{i}$ we consider the line $x_{i}+\left(x^{u}, 0,0\right)$ for $x^{u} \in\left[-\varepsilon^{\theta}, \varepsilon^{\theta}\right]$, and we partition it in intervals of length $\varepsilon / 3$. To each such interval $I_{i, j}$ we associate a point

$$
x_{i, j} \in \cup_{A \in E_{\ell, i}} W_{A}^{0} \cap I_{i, j},
$$

if the intersection is not empty. For each such point $x_{i, j}$, we choose an index $A$ so that $x_{i, j} \in$ $W_{A}^{0}$, and we associate Reeb coordinates $\tilde{\kappa}_{x_{i, j}}$ to $x_{i, j}$ as follows: We require that $x_{i, j}$ is at the origin in the $\tilde{\kappa}_{x_{i, j}}$ coordinates, that $\tilde{\kappa}_{x_{i, j}}\left(W_{A}^{0}\right) \subset\left\{\left(0, x^{s}, x^{0}\right) \mid x^{s}, x^{0} \in \mathbb{R}\right\}$, and that the vector $D_{x_{i, j}} \Phi_{-\ell \tau_{-}}(1,0,0)$ belongs to the unstable cone. Such changes of coordinates exist and are uniformly $\mathcal{C}^{2}$ by $[\mathrm{BL}$, Lemma A.4].

Letting $\mathcal{B}_{\varepsilon}=\left\{\left(x^{u}, x^{s}, x^{0}\right):\left|x^{u}\right| \leq \varepsilon,\left|x^{s}\right| \leq \varepsilon^{\theta},\left|x^{0}\right| \leq \varepsilon^{\theta}\right\}$, we consider for each $x_{i, j}$ the box (in the coordinates $\tilde{\kappa}_{x_{i, j}}$ )

$$
\mathcal{B}_{\varepsilon, i, j}=\tilde{\kappa}_{x_{i, j}}^{-1}\left(\mathcal{B}_{\varepsilon}\right) .
$$

Up to taking larger $c$, we may ensure that the support of the element of the partition of unity corresponding to $x_{i}$ does not intersect the flow and weak-unstable boundaries of $\mathcal{B}_{\varepsilon, i, j}$.

We next control regular pieces intersecting $\mathcal{B}_{\varepsilon}$ :

Lemma 8.5 (Controlling regular pieces intersecting $\mathcal{B}_{\varepsilon}$ ). For each $i, j$ and any $B \in E_{\ell, i}$ so that $W_{B} \cap \mathcal{B}_{\varepsilon, i, j} \neq \emptyset$, if $\varepsilon$ is sufficiently small so that $C \varepsilon^{\theta / 5} \leq 1 / 2$, where $C$ is from Section $\square$, then the flowed leaf $W_{B}^{0} \cap \partial \mathcal{B}_{2 \varepsilon, i, j}$ does not intersect the stable side of $\mathcal{B}_{2 \varepsilon, i, j}$. 
Proof. By construction, for each $\mathcal{B}_{\varepsilon, i, j}$ there is a manifold $W_{A}^{0}$ going through its center and perpendicular to $(1,0,0)$ (in the $\tilde{\kappa}_{x_{i, j}}$ coordinates), for some $A \in E_{\ell, i}$. Let $\tau_{A} \in\left[-\tau_{-}, \tau_{-}\right]$be such that $\widetilde{W}_{A}:=\Phi_{\tau_{A}} W_{A}$ satisfies $\widetilde{W}_{A} \cap I_{i, j}=\left\{\left(x_{A}^{u}, 0,0\right)\right\} \neq \emptyset 37$

Next, consider $B \in E_{\ell, i}$ with $B \neq A$ so that $W_{B}^{0}$ intersects $\mathcal{B}_{\varepsilon, i, j}$, and let $x_{B}^{u}$ be the intersection point with $I_{i, j}$. Again, let $\widetilde{W}_{B}=\Phi_{\tau_{B}} W_{B}$. By construction $\left|x_{A}^{u}-x_{B}^{u}\right| \leq \varepsilon$. Our first goal is to estimate $d\left(\widetilde{W}_{A}, \widetilde{W}_{B}\right)$. To this end we will use the stable version of the fake foliations constructed in Section 66. Observe that each result concerning the unstable curves can be transformed into a result for the stable curves by time reversal. More precisely, we construct a foliation in the neighborhood of $I_{i, j}$ made of stable curves of length $\rho=c \varepsilon^{\theta}$.

To do this, consider $P^{+}\left(\Phi_{\ell \tau_{-}}\left(I_{i, j}\right)\right)$, which is a countable union of homogeneous unstable curves, defined analogously to the set $\widetilde{\mathcal{G}}_{n}(V)$ for a map-stable curve $V$ in the proof of Lemma 3.8 . Note that by construction, both $\widetilde{W}_{A, i}$ and $\widetilde{W}_{B, i}$ can be iterated forward for a time $\ell \tau_{-}$, and $\Phi_{\ell \tau_{-}}\left(\widetilde{W}_{A, i} \cup \widetilde{W}_{B, i}\right) \subset W^{0}$. Thus both $P^{+}\left(\Phi_{\ell \tau_{-}}\left(\widetilde{W}_{A, i}\right)\right)$ and $P^{+}\left(\Phi_{\ell \tau_{-}}\left(\widetilde{W}_{B, i}\right)\right)$ are subcurves of $P^{+}(W)$, and they intersect $P^{+}\left(\Phi_{\ell \tau_{-}}\left(I_{i, j}\right)\right)$.

Now using the (time reversed) construction detailed in Section 6, we choose a seeding foliation, defined on homogeneous subcurves of $P^{+}\left(\Phi_{\ell \tau_{-}}\left(I_{i, j}\right)\right)$, that contains both $P^{+}\left(\Phi_{\ell \tau_{-}}\left(\widetilde{W}_{A, i}\right)\right)$ and $P^{+}\left(\Phi_{\ell \tau_{-}}\left(\widetilde{W}_{B, i}\right)\right)$ as two of its curves. Notice that this choice still allows the seeding foliation to be uniformly $\mathcal{C}^{2}$ since both $P^{+}\left(\Phi_{\ell \tau_{-}}\left(\widetilde{W}_{A, i}\right)\right)$ and $P^{+}\left(\Phi_{\ell \tau_{-}}\left(\widetilde{W}_{B, i}\right)\right)$ are subcurves of $P^{+}(W)$ and since we assumed that $W$ is homogeneous, by definition of $\mathcal{W}^{s}$ and Lemma 3.4, $P^{+}(W)$ is a single homogeneous map-stable curve with uniformly bounded curvature.

By the definition of $\Delta_{\varkappa}$ in Section 6.1, this means that both $\widetilde{W}_{A, i}$ and $\widetilde{W}_{B, i}$ belong to the fake stable foliation in a neighborhood of $I_{i, j}$. Note in particular that $\widetilde{W}_{A, i}$ and $\widetilde{W}_{B, i}$ do not belong to the gaps in the foliation since by construction, they are images of stable curves on which $\Phi_{-\ell \tau_{-}}$is smooth and $A, B \in E_{\ell, i}$. Also, they are guaranteed to have length at least $\rho=c \varepsilon^{\theta}$ because, having their indices in $E_{\ell, i}$, they completely cross $B_{c \varepsilon^{\theta}}\left(x_{i}\right)$. In the coordinates used in Section 6 (with the stable and unstable interchanged), let $\left(G\left(x_{A}^{u}, x^{s}\right), x^{s}, H\left(x_{A}^{u}, x^{s}\right)\right)$ and $\left(G\left(x_{B}^{u}, x^{s}\right), x^{s}, H\left(x_{B}^{u}, x^{s}\right)\right)$ be the curves $\widetilde{W}_{A, i}, \widetilde{W}_{B, i}$, respectively. By properties (ii) and (vi) of the fake foliation, it follows that

$$
\begin{aligned}
\left|G\left(x_{B}^{u}, x^{s}\right)-G\left(x_{A}^{u}, x^{s}\right)\right| & =\left|\int_{x_{A}^{u}}^{x_{B}^{u}} \partial_{x^{u}} G\left(u, x^{s}\right) d u\right|=\left|\int_{x_{A}^{u}}^{x_{B}^{u}}\left[1+\int_{0}^{x^{s}} \partial_{x^{s}} \partial_{x^{u}} G(u, s) d s\right] d u\right| \\
& \leq \int_{x_{A}^{u}}^{x_{B}^{u}}\left[1+C \varepsilon^{\theta / 5}\right] d u \leq \varepsilon\left(1+C \varepsilon^{\theta / 5}\right) \leq(3 / 2) \varepsilon<2 \varepsilon
\end{aligned}
$$

where we have chosen $\varepsilon$ sufficiently small that $C \varepsilon^{\theta / 5} \leq 1 / 2$. Analogously, by properties (ii) and (v) of the foliation,

$$
\left|H\left(x_{B}^{u}, x^{s}\right)-H\left(x_{A}^{u}, x^{s}\right)\right|=\left|\int_{x_{A}^{u}}^{x_{B}^{u}} \int_{0}^{x^{s}} \partial_{x^{s}} \partial_{x^{u}} H(u, s) d s d u\right| \leq C_{\#} \varepsilon^{1+\theta} .
$$

Hence $W_{B}^{0}$ cannot intersect the stable boundary of $\mathcal{B}_{2 \varepsilon, i, j}$, again assuming $C_{\#} \varepsilon^{\theta} \leq 3 / 2$.

Remark 8.6. The above estimates on the distances of nearby manifolds can be applied to the "central" manifolds of the boxes as well. This readily implies that the covering $\left\{\mathcal{B}_{\varepsilon, i, j}\right\}$ has a uniformly bounded number of overlaps.

37 When no confusion arises, to ease notation, we will identify $\tilde{\kappa}_{x_{i, j}}\left(W_{A}^{0}\right)$ and $W_{A}^{0}$. 
After this preparation, we can summarise where we stand: From (8.4), (8.11), (8.14) and assumption (8.12) we have

$$
\begin{aligned}
\int_{W} \psi \cdot\left(\mathcal{R}(z)^{m}(f)\right) d m_{W}=\sum_{\ell, i \in \mathbb{N}} \sum_{A \in A_{\ell, i}} \int_{-\tau_{-}}^{\tau_{-}} p_{m, \ell, z}(s) \int_{W_{A, i}} J_{\ell \tau_{-}}^{s} \cdot \psi \circ \Phi_{\ell \tau_{-}} \cdot \phi_{\varepsilon, i} \cdot \mathcal{L}_{s} f \\
=\sum_{\ell \geq \ell_{0}} \sum_{i=1}^{\infty} \sum_{j} \sum_{A \in E_{\ell, i, j}} \int_{-\tau_{-}}^{\tau_{-}} p_{m, \ell, z}(s) \int_{W_{A, i}} J_{\ell \tau_{-}}^{s} \cdot \psi \circ \Phi_{\ell \tau_{-}} \cdot \phi_{\varepsilon, i} \cdot \mathcal{L}_{s} f \\
\quad+\mathcal{O}\left(\varepsilon^{\frac{\theta}{2}} a^{-m}|f|_{\infty}|\psi|_{\infty}\right),
\end{aligned}
$$

where $E_{\ell, i, j} \subset\left\{A \in E_{\ell, i}: W_{A, i} \cap \mathcal{B}_{\varepsilon, i, j} \neq \emptyset\right\}, E_{\ell, i, j} \cap E_{\ell, i, k} \neq \emptyset$ implies $j=k$, and $\cup_{j} E_{\ell, i, j}=E_{\ell, i}$. To continue, we need some notation that, for each $\ell, i, j$, is better stated in the above mentioned Reeb charts $\tilde{\kappa}_{x_{i, j}}$. In fact, from now on, we identify the coordinate charts and the manifold notation. For each $A \in E_{\ell, i, j}$, we now view $W_{A, i}^{0} \cap \mathcal{B}_{c \varepsilon}$ (directly) as the graph of $\mathbb{W}_{A}^{0}\left(x^{s}, x^{0}\right):=\mathbb{W}_{A}\left(x^{s}\right)+\left(0,0, x^{0}\right)$ where

$$
\mathbb{W}_{A}\left(x^{s}\right):=\left(M_{A}\left(x^{s}\right), x^{s}, N_{A}\left(x^{s}\right)\right), \quad\left|x^{s}\right| \leq c \varepsilon^{\theta},
$$

and $M_{A}$ and $N_{A}$ are uniformly $\mathcal{C}^{2}$ functions. By the same exact arguments used in the proof of Lemma 8.5, we have 39

$$
\left|M_{A}^{\prime}\right| \leq C_{\#} \varepsilon^{1-\frac{4 \theta}{5}}
$$

Since both $\boldsymbol{\alpha}$ and $\mathbf{d} \boldsymbol{\alpha}$ are invariant under the flow, and the manifolds are the images of manifolds with tangent space in the kernel of both forms, we have that

$$
N_{A}^{\prime}\left(x^{s}\right)=x^{s} M_{A}^{\prime}\left(x^{s}\right) .
$$

With the above notation, the integrals in equation (8.20) can be rewritten as

$$
\int_{-\tau_{-}}^{\tau_{-}} p_{m, \ell, z}(s) \int_{W_{A, i}} \psi \circ \Phi_{\ell \tau_{-}} \cdot J_{\ell \tau_{-}}^{s} \cdot \phi_{\varepsilon, i} \cdot \mathcal{L}_{s} f d s=\int_{\mathcal{Q}_{c \varepsilon} \theta} p_{A} \cdot \psi_{A} \cdot \bar{J}_{A, \ell \tau_{-}}^{s} \cdot \phi_{\varepsilon, A} \cdot f_{A} d x^{s} d x^{0}
$$

where $\mathcal{Q}_{\delta}=\left\{\left(x^{s}, x^{0}\right):\left|x^{s}\right| \leq \delta,\left|x^{0}\right| \leq \delta\right\}$ and

$$
\begin{aligned}
& p_{A}\left(x^{s}, x^{0}\right)=p_{m, \ell, z}\left(-x^{0}\right), \quad \phi_{\varepsilon, A}\left(x^{s}, x^{0}\right)=\phi_{\varepsilon, i} \circ \mathbb{W}_{A}^{0}\left(x^{s}, x^{0}\right) \cdot\left\|\mathbb{W}_{A}^{\prime}\left(x^{s}\right)\right\|, \\
& \psi_{A}\left(x^{s}, x^{0}\right)=\psi \circ \Phi_{\ell \tau_{-}} \circ \mathbb{W}_{A}\left(x^{s}\right), \quad \bar{J}_{A, \ell \tau_{-}}^{s}\left(x^{s}, x^{0}\right)=J_{\ell \tau_{-}}^{s} \circ \mathbb{W}_{A}\left(x^{s}\right), \\
& f_{A}\left(x^{s}, x^{0}\right)=f \circ \mathbb{W}_{A}^{0}\left(x^{s}, x^{0}\right) .
\end{aligned}
$$

Our strategy will be based on the fact that an oscillatory integral of a Lipschitz function is small. Unfortunately, the integrands above are not Lipschitz. To deal with this, let

$$
\Xi_{\ell, A, i, j}=\psi_{A}(0,0) \cdot Z_{\ell, A, i},
$$

recalling $Z_{\ell, A, i}$ defined by (8.8). By Lemma 3.5 and the fact that $|\psi|_{\mathcal{C}^{\alpha}(W)} \leq 1$, we have

$$
\left.\left.\left|\psi_{A} \cdot \bar{J}_{A, \ell \tau_{-}}^{s}-\Xi_{\ell, A, i, j}\right| W_{A, i}\right|^{-1}\right|_{\infty} \leq C_{\#} \varepsilon^{\alpha \theta} \frac{Z_{\ell, A, i}}{\left|W_{A, i}\right|}
$$

\footnotetext{
38 Some manifolds can be attributed either to a box or to an adjacent box and a choice can be made to resolve the ambiguity.

39 The manifold $W_{B}$ in the proof of Lemma 8.5 corresponds here to the central manifold of the box, which in the current coordinates reads $\left\{\left(0, x^{s}, 0\right)\right\}$, while $M_{A}\left(x^{s}\right)$ corresponds to $G\left(x_{A}^{u}, x^{s}\right)$.
} 
Therefore, using (8.25) in (8.23) and substituting it in (8.20), yields

$$
\begin{aligned}
\int_{W} \psi \mathcal{R}(z)^{m}(f) d m_{W}= & \sum_{\ell \geq \ell_{0}} \sum_{i, j} \sum_{A \in E_{\ell, i, j}} \frac{\Xi_{\ell, A, i, j}}{\left|W_{A, i}\right|} \int_{\mathcal{Q}_{c \varepsilon} \theta} p_{A} \cdot \phi_{\varepsilon, A} \cdot f_{A} \\
& +\mathcal{O}\left(|f|_{\infty}|\psi|_{\infty} \varepsilon^{\alpha \theta} a^{-m}\right)
\end{aligned}
$$

since $\alpha \leq 1 / 3$. Recalling $p(s)=p(-s)$, we introduce (to ease notation we suppress some indices):

$$
\begin{aligned}
\mathbf{G}_{\ell, m, i, A}\left(x^{s}, x^{0}\right)= & p\left(x^{0}\right) \frac{\left(\ell \tau_{-}-x^{0}\right)^{m-1}}{\left|W_{A, i}\right|(m-1) !} e^{-z \ell \tau_{-}+a x^{0}} \\
& \cdot \phi_{\varepsilon, i}\left(M_{A}\left(x^{s}\right), x^{s}, N_{A}\left(x^{s}\right)-x^{0}\right) \cdot \Theta_{A}\left(x^{s}\right),
\end{aligned}
$$

where $\Theta_{A} d s \wedge d x^{s}$ is the volume form on $W_{A}^{0}$ in the coordinates $\mathbb{W}_{A}^{0}$. Note that

$$
\begin{aligned}
\left|\mathbf{G}_{\ell, m, i, A}\right|_{\infty} & \leq C_{\#} \varepsilon^{-\theta} \frac{\left(\ell \tau_{-}\right)^{m-1}}{(m-1) !} e^{-a \ell \tau_{-}} \\
\left|\mathbf{G}_{\ell, m, i, A}\right|_{\text {Lip }} & \leq C_{\#} \varepsilon^{-2 \theta} \frac{\left(\ell \tau_{-}\right)^{m-1}}{(m-1) !} e^{-a \ell \tau_{-}} .
\end{aligned}
$$

Indeed, by construction $\left|W_{A, i}\right| \geq \varepsilon^{\theta}$, and aside from $\ell$, the only large contribution to the Lipschitz norm comes from $\phi_{\varepsilon, A}$ (which only differs from $\phi_{\varepsilon, i}$ by the uniformly smooth change of coordinates $\left.\mathbb{W}_{A}^{0}\right)$, and $p$ and is of order $\varepsilon^{-\theta}$.

With the above notation we can write,

$$
\begin{aligned}
& \int_{W} \psi \mathcal{R}(z)^{m}(f) d m_{W}=\mathcal{O}\left(|f|_{\infty}|\psi|_{\infty} \varepsilon^{\alpha \theta} a^{-m}\right) \\
& +\sum_{\ell \geq \ell_{0}} \sum_{i, j} \sum_{A \in E_{\ell, i, j}} \Xi_{\ell, A, i, j} \int_{\mathcal{Q}_{c \varepsilon} \theta} e^{i b x^{0}} \mathbf{G}_{\ell, m, i, A}\left(x^{s}, x^{0}\right) f\left(\mathbb{W}_{A}\left(x^{s}\right)+\left(0,0, x^{0}\right)\right) .
\end{aligned}
$$

At this point, we would like to compare different manifolds in the same box by sliding them along an approximate unstable direction. For this we fix $\ell, i, j$ and use the approximate unstable fibres $\gamma_{i, j, \varepsilon}^{\varkappa}$ constructed in Section 6 (the parameter $\varkappa$ represents the maximum time in $\mathcal{F}_{\varkappa, x_{0}}$ there). In short, for each coordinate $\tilde{\kappa}_{x_{i, j}}$, we can construct a Lipschitz foliation in $\mathcal{B}_{c \varepsilon}$ in a

$$
\rho=\varepsilon^{\varsigma}
$$

neighborhood of $W_{*}=\left\{\left(0, x^{s}, x^{0}\right)\left|x^{s}\right| \leq c \varepsilon^{\theta},\left|x^{0}\right| \leq c \varepsilon^{\theta}\right\}$. In order to have the foliation defined in all $\mathcal{B}_{c \varepsilon}$ we need $\varsigma<1$, while for the foliation to have large part where it can be smoothly iterated backward as needed it is necessary that $\varsigma>\theta$. We thus impose

$$
\theta<\varsigma<1 \text {. }
$$

The foliation constructed in Section [6 can be described by the coordinate change

$$
\mathbb{F}_{i, j, \varkappa}\left(x^{u}, x^{s}, x^{0}\right)=\left(x^{u}, G_{i, j, \varkappa}\left(x^{u}, x^{s}\right), H_{i, j, \varkappa}\left(x^{u}, x^{s}\right)+x^{0}\right) .
$$

Also, in equation (8.49) it will be essential that $\varkappa$ be large enough. It turns out that

$$
\varkappa=10 m a^{-1}
$$


suffices. The leaf $\gamma_{i, j, \varepsilon}^{\varkappa}\left(x^{s}, x^{0}\right)$ is thus the graph of $\mathbb{F}_{i, j, \varkappa}\left(\cdot, x^{s}, x^{0}\right)$. By construction, we require that the vector $(1,0,0)$ is tangent to the curve in the foliation passing through $(0,0,0)$. Thus 40

$$
\begin{aligned}
G_{i, j, \varkappa}(0,0) & =H_{i, j, \varkappa}(0,0)=\partial_{x^{u}} G_{i, j, \varkappa}(0,0)=\partial_{x^{u}} H_{i, j, \varkappa}(0,0)=0 \\
\left|G_{i, j, \varkappa}\left(x^{u}, 0\right)\right| & \leq C \rho^{2}, \quad\left|H_{i, j, \varkappa}\left(x^{u}, 0\right)\right| \leq C \rho^{2} .
\end{aligned}
$$

For $A \in E_{\ell, i, j}$, we consider the holonomy

$$
\mathbf{h}_{i, j, A, \varkappa}: W_{A} \cap \mathcal{B}_{\varepsilon} \rightarrow W_{*}
$$

defined by $\{z\}=\gamma_{i, j, \varepsilon}^{\varkappa}\left(\mathbf{h}_{i, j, A, \varkappa}(\{z\})\right) \cap W_{A}$. Recalling (8.21), we shall use the notation

$$
\mathbf{h}_{i, j, A, \varkappa} \circ \mathbb{W}_{A}\left(x^{s}\right)=\left(0, \mathbf{h}_{A}^{s}\left(x^{s}\right), \mathbf{h}_{A}^{0}\left(x^{s}\right)\right),
$$

where, by construction and provided $c$ has been chosen large enough, for all $\left|x^{s}\right| \leq \varepsilon^{\theta}$,

$$
\left|\mathbf{h}_{A}^{s}(0)\right| \leq C \varepsilon^{2},\left|\mathbf{h}_{A}^{0}(0)-N_{A}(0)\right| \leq C \varepsilon^{2},\left|\mathbf{h}_{A}^{s}\left(x^{s}\right)\right| \leq c \varepsilon^{\theta},\left|\mathbf{h}_{A}^{0}\left(x^{s}\right)\right| \leq c \varepsilon^{\theta} .
$$

In other words, $\mathbb{F}_{i, j, \varkappa}\left(M_{A}\left(x^{s}\right), \mathbf{h}_{A}^{s}\left(x^{s}\right), \mathbf{h}_{A}^{0}\left(x^{s}\right)\right)=\mathbb{W}_{A}\left(x^{s}\right)$, that is

$$
G_{i, j, \varkappa}\left(M_{A}\left(x^{s}\right), \mathbf{h}_{A}^{s}\left(x^{s}\right)\right)=x^{s}, \quad H_{i, j, \varkappa}\left(M_{A}\left(x^{s}\right), \mathbf{h}_{A}^{s}\left(x^{s}\right)\right)+\mathbf{h}_{A}^{0}\left(x^{s}\right)=N_{A}\left(x^{s}\right) .
$$

We shall need the following bounds on the regularity of $\mathbf{h}_{A}^{s}$. The proof of Lemma 8.7 is provided in Appendix B.

Lemma 8.7. There exists $C_{\#}>0$ such that for each $i, j, \ell$, and every $A \in E_{\ell, i, j}$, we have

$$
\left|\mathbf{h}_{A}^{s}\left(x^{s}\right)-x^{s}\right|+\left|\left(\mathbf{h}_{A}^{s}\right)^{-1}\left(x^{s}\right)-x^{s}\right| \leq C_{\#} \varepsilon^{1-\frac{4}{5} \varsigma+\theta}, \quad\left|1-\left(\mathbf{h}_{A}^{s}\right)^{\prime}\right| \leq C_{\#} \varepsilon^{1-\frac{4}{5} \varsigma} .
$$

Next, remember that the fibers $\gamma_{i, j, \varepsilon}^{\varkappa}$ in the domain $\Delta_{\varkappa}$ can be iterated backward a time $\varkappa$ and still remain in the unstable cone. In the following we will use the notation

$$
\partial_{\varkappa, i} f=\sup _{\left(x^{s}, x^{0}\right) \in \Delta \varkappa} \operatorname{ess-sup}_{\left|x^{u}\right| \leq \varepsilon}\left|\left\langle\partial_{x^{u}} \mathbb{F}_{i, j, \varkappa}\left(x^{u}, x^{s}, x^{0}\right),(\nabla f) \circ \mathbb{F}_{i, j, \varkappa}\left(x^{u}, x^{s}, x^{0}\right)\right\rangle\right| .
$$

With the above construction and notations, using (8.28), (8.34), (8.35) and Lemma 8.7, we can continue our estimate left at (8.29) (recalling (8.33))

$$
\begin{aligned}
& \int_{\mathcal{Q}_{c \varepsilon} \theta} d x^{0} d x^{s} e^{i b x^{0}} \mathbf{G}_{\ell, m, i, A}\left(x^{s}, x^{0}\right) f\left(\mathbb{W}_{A}\left(x^{s}\right)+\left(0,0, x^{0}\right)\right) \\
& =\int_{\mathcal{Q}_{c \varepsilon} \theta} d x^{0} d x^{s} e^{i b x^{0}} \mathbf{G}_{\ell, m, i, A}\left(x^{s}, x^{0}\right) f\left(0, \mathbf{h}_{A}^{s}\left(x^{s}\right), \mathbf{h}_{A}^{0}\left(x^{s}\right)+x^{0}\right) \\
& \quad+\mathcal{O}\left(\varepsilon^{1+\theta} \partial_{\varkappa, i} f+\varepsilon^{\varsigma}|f|_{\infty}\right) \cdot \frac{\left(\ell \tau_{-}\right)^{m-1}}{(m-1) !} e^{-a \ell \tau_{-}} \\
& =\int_{\mathcal{Q}_{c \varepsilon} \theta} d x^{0} d x^{s} e^{i b\left(x^{0}-\boldsymbol{\omega}_{A}\left(x^{s}\right)\right)} \frac{\mathbf{G}_{\ell, m, i, A}^{*}\left(x^{s}, x^{0}\right)}{\left|\left(\mathbf{h}_{A}^{s}\right)^{\prime} \circ\left(\mathbf{h}_{A}^{s}\right)^{-1}\left(x^{s}\right)\right|} f\left(0, x^{s}, x^{0}\right) \\
& \quad+\mathcal{O}\left(\varepsilon^{1+\theta} \partial_{\varkappa, i} f+\varepsilon^{\varsigma}|f|_{\infty}\right) \cdot \frac{\left(\ell \tau_{-}\right)^{m-1}}{(m-1) !} e^{-a \ell \tau_{-}} \\
& =\int_{\mathcal{Q}_{c \varepsilon} \theta} d x^{0} d x^{s} e^{i b\left(x^{0}-\omega_{A}\left(x^{s}\right)\right)} \mathbf{G}_{\ell, m, i, A}^{*}\left(x^{s}, x^{0}\right) f\left(0, x^{s}, x^{0}\right) \\
& \quad+\mathcal{O}\left(\varepsilon^{1+\theta} \partial_{\varkappa, i} f+\left(\varepsilon^{\varsigma}+\varepsilon^{1-4 \varsigma / 5+\theta}\right)|f|_{\infty}\right) \cdot \frac{\left(\ell \tau_{-}\right)^{m-1}}{(m-1) !} e^{-a \ell \tau_{-}},
\end{aligned}
$$

\footnotetext{
40 We cannot require that the leaf $\left(x^{u}, 0,0\right)$ belongs to the foliation, but the proof of [BL, Lemma A.4] ensures that we can obtain the desired tangency.
} 
where in the first equality, we have expanded $f$ at the reference manifold $W_{*}$, using $\partial_{\varkappa, i} f$ on $\Delta_{\varkappa}$ and property (iv) of the foliation on $W_{*} \backslash \Delta_{\varkappa}$; in the second equality, we have changed variables, $x^{0} \mapsto x^{0}-h_{A}^{0}\left(x^{s}\right)$ and then $x^{s} \mapsto\left(h_{A}^{s}\right)^{-1}\left(x^{s}\right)$; and in the third equality we have used Lemma 8.7, setting $\boldsymbol{\omega}_{A}\left(x^{s}\right)=\mathbf{h}_{A}^{0} \circ\left(\mathbf{h}_{A}^{s}\right)^{-1}\left(x^{s}\right)$, and

$$
\mathbf{G}_{\ell, m, i, A}^{*}\left(x^{s}, x^{0}\right)=\mathbf{G}_{\ell, m, i, A}\left(\left(\mathbf{h}_{A}^{s}\right)^{-1}\left(x^{s}\right), x^{0}-\boldsymbol{\omega}_{A}\left(x^{s}\right)\right) .
$$

Note that Lemma 8.7 and (8.28) imply that there exists $C_{\#}>0$ such that, for all $A$,

$$
\left|\mathbf{G}_{\ell, m, i, A}^{*}\right|_{\infty} \leq C_{\#} \varepsilon^{-\theta} \frac{\left(\ell \tau_{-}\right)^{m-1}}{(m-1) !} e^{-a \ell \tau_{-}}, \quad\left|\mathbf{G}_{\ell, m, i, A}^{*}\right|_{\operatorname{Lip}} \leq C_{\#} \varepsilon^{-2 \theta} \frac{\left(\ell \tau_{-}\right)^{m-1}}{(m-1) !} e^{-a \ell \tau_{-}} .
$$

At last, we can substitute (8.37) into (8.29): We note that (8.8), (8.24) imply

$$
\sum_{i, j} \sum_{A \in E_{\ell, A, i, j}} \Xi_{\ell, A, i, j} \leq C_{\#},
$$

and use the Schwarz inequality (first with respect to the integrals and then with respect to the sum on $i, j)$ together with the trivial identity $\left|\sum_{A} z_{A}\right|^{2}=\left(\sum_{A} z_{A}\right)\left(\sum_{B} \bar{z}_{B}\right)$ on sums of complex numbers, to obtain 41

$$
\begin{aligned}
& \left|\int_{W} \psi \mathcal{R}(z)^{m}(f) d m_{W}\right| \leq C_{\#}\left[\left(\varepsilon^{\alpha \theta}+\varepsilon^{1-4 \varsigma / 5}+\varepsilon^{\varsigma-\theta}\right)|f|_{\infty}+\varepsilon \sup _{i} \partial_{\varkappa, i} f\right] a^{-m} \\
& +C_{\#} \sum_{\ell \geq \ell_{0}} \sum_{i, j}|f|_{\infty} \varepsilon^{\theta}\left\{\sum_{A, B \in E_{\ell, i, j}} \Xi_{\ell, A, i, j} \Xi_{\ell, B, i, j} \int_{\mathcal{Q}_{c \varepsilon} \theta} e^{i b\left[\boldsymbol{\omega}_{A}-\boldsymbol{\omega}_{B}\right]}\left[\mathbf{G}_{\ell, m, i, A}^{*} \cdot \overline{\mathbf{G}_{\ell, m, i, B}^{*}}\right]\right\}^{\frac{1}{2}} \\
& \leq C_{\#}\left[\left(\varepsilon^{\alpha \theta}+\varepsilon^{1-4 \varsigma / 5}+\varepsilon^{\varsigma-\theta}\right)|f|_{\infty}+\varepsilon \sup _{i} \partial_{\varkappa, i} f\right] a^{-m} \\
& +C_{\#} \sum_{\ell \geq \ell_{0}}|f|_{\infty} \varepsilon^{-\frac{1}{2}}\left\{\sum_{i, j} \sum_{A, B \in E_{\ell, i, j}} Z_{\ell, A, i} Z_{\ell, B, i}\left|\int_{\mathcal{Q}_{c \varepsilon} \theta} e^{i b\left[\boldsymbol{\omega}_{A}-\boldsymbol{\omega}_{B}\right]}\left[\mathbf{G}_{\ell, m, i, A}^{*} \overline{\mathbf{G}_{\ell, m, i, B}^{*}}\right]\right|\right\}^{\frac{1}{2}} .
\end{aligned}
$$

In view of the cancellation argument needed to conclude the present proof of Lemma 8.1, we need a fundamental, but technical, result whose proof can be found in Appendix B.

Lemma 8.8 (Oscillatory integral). We have

$$
\inf _{x^{s}}\left|\partial_{x^{s}}\left[\boldsymbol{\omega}_{A}-\boldsymbol{\omega}_{B}\right]\left(x^{s}\right)\right| \geq C_{\#} d\left(W_{A}, W_{B}\right) .
$$

In addition, provided that

$$
0<\varpi \leq \frac{\theta}{5} \text { and } 4 \varsigma / 5+\varpi(7+11 \varsigma / 15) \leq 1
$$

we have

$$
\left|\boldsymbol{\omega}_{A}-\boldsymbol{\omega}_{B}\right|_{\mathcal{C}^{1+\varpi}\left(\mathcal{Q}_{c \varepsilon} \theta\right)} \leq C_{\#} \varepsilon
$$

which implies

$$
\begin{aligned}
& \left|\int_{\left|x^{s}\right| \leq c \varepsilon^{\theta}} d x^{s} e^{i b\left(\boldsymbol{\omega}_{A}\left(x^{s}\right)-\boldsymbol{\omega}_{B}\left(x^{s}\right)\right)} \mathbf{G}_{\ell, m, i, A}^{*} \overline{\mathbf{G}_{\ell, m, i, B}^{*}}\right|_{\infty} \\
& \leq C_{\#} \frac{\left(\ell \tau_{-}\right)^{2 m-2}}{[(m-1) !]^{2}} e^{-2 a \ell \tau_{-}} \varepsilon^{-\theta}\left[\frac{\varepsilon}{d\left(W_{A}, W_{B}\right)^{1+\varpi} b^{\varpi}}+\frac{1}{\varepsilon^{\theta} d\left(W_{A}, W_{B}\right) b}\right] .
\end{aligned}
$$

\footnotetext{
41 Remember that $i$ runs from 1 to $C_{\#} \varepsilon^{-3 \theta}$, while $j$ from 1 to $C_{\#} \varepsilon^{\theta-1}$, so, all in all, the sum over $i, j$ consists of $C_{\#} \varepsilon^{-1-2 \theta}$ terms.
} 
Note that the proof of the $\mathcal{C}^{1+\varpi}$ estimates on the holonomy in [BL, Lemma 6.6] used a Hölder bound on $\partial_{x^{u}} \partial_{x^{s}} G$ (called (6) in [BL, App. D]) that is not available in the present context. Yet, it turns out that the four-point estimate (vii) from Section 6] suffices to prove the bound (8.43) on $\boldsymbol{\omega}_{A}-\boldsymbol{\omega}_{B}$.

We have now all the ingredients to conclude the proof of Lemma 8.1. It is convenient to introduce a parameter $\vartheta>1$ and to define

$$
\begin{aligned}
& \mathbb{E}_{\ell, i, j}^{\text {close }}=\left\{(A, B) \in E_{\ell, i, j} \times E_{\ell, i, j}: d\left(W_{A}, W_{B}\right) \leq \varepsilon^{\vartheta}\right\} \\
& \mathbb{E}_{\ell, i, j}^{\mathrm{far}}=\left\{(A, B) \in E_{\ell, i, j} \times E_{\ell, i, j}: d\left(W_{A}, W_{B}\right)>\varepsilon^{\vartheta}\right\} .
\end{aligned}
$$

To estimate the sum on $\mathbb{E}_{\ell, i, j}^{\text {close }}$, we will need the following lemma (proved in Appendix B).

Lemma 8.9. For each $\ell \geq \ell_{0}, i \in \mathbb{N}$ and $A \in E_{\ell, i}$, the following estimate holds,

$$
\sum_{\left\{(B, i): i \in \mathbb{N}, B \in E_{\ell, i}, d\left(W_{B, i}, W_{A, i}^{0}\right) \leq \rho_{*}\right\}} Z_{\ell, B, i} \leq C_{\#}\left[\varepsilon^{\theta} \sqrt{\rho_{*}}+\lambda^{\ell \tau_{-} / 2}\right],
$$

where $\lambda<1$ is from Lemma 3.8 .

Thus, by the above Lemma with $\rho_{*}=\varepsilon^{\vartheta}$, we have

$$
\begin{aligned}
\sum_{(A, B) \in \mathbb{E}_{\ell, i, j}^{\text {close }}} Z_{\ell, A, i} Z_{\ell, B, i} & =\sum_{A \in E_{\ell, i, j}} Z_{\ell, A, i} \sum_{(A, B) \in \mathbb{E}_{\ell, i, j}^{\text {close }}} Z_{\ell, B, i} \\
& \leq C_{\#} \sum_{A \in E_{\ell, i, j}} Z_{\ell, A, i}\left[\varepsilon^{\theta+\vartheta / 2}+\lambda^{\ell \tau-/ 2}\right] .
\end{aligned}
$$

Next, we assume (possibly strengthening assumption (8.12)),

$$
\lambda^{\ell_{0} \tau_{-} / 2}=\left(e^{-m}\right)^{\frac{\ln \lambda^{-1}}{2 a e^{2}}} \leq \varepsilon^{\theta+\vartheta / 2} .
$$

Hence, using estimate (8.45), for $\ell \geq \ell_{0}$,

$$
\sum_{i, j} \sum_{(A, B) \in \mathbb{E}_{\ell, i, j}^{\text {close }}} Z_{\ell, A, i} Z_{\ell, B, i} \leq C_{\#}|W| \varepsilon^{\theta+\vartheta / 2}
$$

To conclude, we want to use estimate (8.40), applied to $\mathcal{R}(z)^{m} f$ rather than to $f$. The reason for this is to obtain an estimate in terms of the $H_{\infty}^{1}$ norm, but with a very small factor in front. More precisely, we will in fact estimate,

$$
\int_{W} \mathcal{R}(z)^{2 m} f \psi d m_{W}=\int_{W} \mathcal{R}(z)^{m} g_{\varkappa, 1} \psi d m_{W}+\int_{W} \mathcal{R}(z)^{m} g_{\varkappa, 2} \psi d m_{W},
$$

where $g_{\varkappa, 1}$ and $g_{\varkappa, 2}$ are defined by

$$
\mathcal{R}(z)^{m} f=\int_{0}^{\varkappa} \frac{s^{m-1}}{(m-1) !} e^{-z s} \mathcal{L}_{s} f d s+\int_{\varkappa}^{\infty} \frac{s^{m-1}}{(m-1) !} e^{-z s} \mathcal{L}_{s} f d s=: g_{\varkappa, 1}+g_{\varkappa, 2} .
$$


To estimate the second term in (8.48), recalling (5.3) and (8.31), notice that

$$
\begin{aligned}
\left|g_{\varkappa, 2}\right|_{\infty} & \leq|f|_{\infty} \int_{\varkappa}^{\infty} \frac{s^{m-1}}{(m-1) !} e^{-a s} d s=|f|_{\infty} e^{-10 m} a^{-m} \sum_{k=0}^{m-1} \frac{(10 m)^{k}}{k !} \\
& \leq|f|_{\infty} e^{-10 m} a^{-m} \frac{(10 m)^{m-1}}{(m-1) !} \sum_{k=0}^{m-1} \frac{(m-1) \cdots k}{(10 m)^{m-1-k}} \\
& \leq|f|_{\infty} e^{-10 m} a^{-m} \frac{(10 m)^{m}}{m !} \sum_{k=0}^{m-1} 10^{-m+k} \leq C_{\#}|f|_{\infty} a^{-m} e^{-6 m} .
\end{aligned}
$$

Thus,

$$
\left|\mathcal{R}(z)^{m} g_{\varkappa, 2}\right|_{\infty} \leq a^{-m}\left|g_{\varkappa, 2}\right|_{\infty} \leq C_{\#} a^{-2 m} e^{-6 m}|f|_{\infty} \leq C_{\#} a^{-2 m} \varepsilon^{3 \theta}|f|_{\infty},
$$

where in the last inequality we have used condition (8.12), $e^{-m} \leq \varepsilon^{\theta / 2}$.

To estimate the first term in (8.48), we apply the bounds derived throughout this section, in particular (8.31) and (8.36), with $g_{\varkappa, 1}$ in place of $f$, noting that $\left|g_{\varkappa, 1}\right|_{\infty} \leq a^{-m}|f|_{\infty}$ and

$$
\begin{aligned}
\left|\partial_{\varkappa, i}\left(g_{\varkappa, 1}\right)\right| & \leq \int_{0}^{\varkappa} \frac{s^{m-1}}{(m-1) !} e^{-a s} \partial_{\varkappa, i}\left(f \circ \Phi_{-s}\right) d s \\
& \leq C_{\#}|\nabla f|_{\infty} \int_{0}^{\infty} \frac{s^{m-1}}{(m-1) !} e^{-(a+\ln \Lambda) s} d s \leq C_{\#}(a+\ln \Lambda)^{-m}|f|_{H_{\infty}^{1}} .
\end{aligned}
$$

Thus, by using (8.39) and (8.47) to estimate the sum over $\mathbb{E}_{\ell, i, j}^{\text {close }}$ and Lemma 8.8 to estimate the sum over $\mathbb{E}_{\ell, i, j}^{\mathrm{far}}$ in (8.40), we obtain

$$
\begin{aligned}
& \left|\int_{W} \psi \mathcal{R}(z)^{2 m}(f) d m_{W}\right| \leq\left|\int_{W} \psi \mathcal{R}(z)^{m}\left(g_{\varkappa, 1}\right) d m_{W}\right|+\left|\int_{W} \psi \mathcal{R}(z)^{m}\left(g_{\varkappa, 2}\right) d m_{W}\right| \\
& \leq C_{\#} \sum_{\ell \geq \ell_{0}}\left|g_{\varkappa, 1}\right|_{\infty} \frac{e^{-a \ell \tau_{-}}\left(\ell \tau_{-}\right)^{m-1}}{(m-1) ! \varepsilon^{\frac{1}{2}}}\left[\frac{\varepsilon^{-\vartheta(1+\varpi)+1}}{b^{\varpi}}+\frac{\varepsilon^{-\theta-\vartheta}}{b}+\varepsilon^{\frac{\vartheta}{2}+\theta}\right]^{\frac{1}{2}} \\
& \quad+C_{\#}\left[\left(\varepsilon^{\alpha \theta}+\varepsilon^{1-4 \varsigma / 5}+\varepsilon^{\varsigma-\theta}\right)\left|g_{\varkappa, 1}\right|_{\infty}+\varepsilon \sup _{i} \partial_{\varkappa, i}\left(g_{\varkappa, 1}\right)\right] a^{-m}+\left|\mathcal{R}(z)^{m} g_{\varkappa, 2}\right|_{\infty} \\
& \leq C_{\#} a^{-2 m}\left(\frac{\varepsilon^{-\frac{(1+\varpi) \vartheta+2 \theta}{2}}}{b^{\frac{w}{2}}}+\frac{\varepsilon^{-\frac{1+3 \theta+\vartheta}{2}}}{b^{\frac{1}{2}}}+\varepsilon^{\frac{\vartheta-2 \theta-2}{4}}+\varepsilon^{\alpha \theta}+\varepsilon^{1-4 \varsigma / 5}+\varepsilon^{\varsigma-\theta}+\varepsilon^{3 \theta}\right)|f|_{\infty} \\
& \quad+C_{\#} a^{-2 m} \varepsilon\left(1+a^{-1} \ln \Lambda\right)^{-m}|f|_{H_{\infty}^{1}},
\end{aligned}
$$

where we have used the fact that

$$
\sum_{\ell \geq \ell_{0}} e^{-a \ell \tau_{-}} \frac{\left(\ell \tau_{-}\right)^{m-1}}{(m-1) !} \leq C \frac{a^{-m}}{\tau_{-}} \leq C^{\prime} a^{-m} \varepsilon^{-\theta} .
$$

Note that if we choose $\varpi=\frac{1}{40}$, then condition (8.42) is satisfied for each $\frac{1}{3} \leq \theta<\varsigma \leq 1$. Thus all the conditions imposed along the computation are

$$
0<\theta<\varsigma<1 ; \vartheta>2+2 \theta ; \lambda^{\ell_{0} \tau_{-} / 2}<\varepsilon^{\theta+\vartheta / 2} .
$$


If we choose $\vartheta=2+4 \theta, b=\varepsilon^{-\frac{(1+\varpi) \vartheta+2(1+\alpha) \theta}{\varpi}}, \theta=\frac{1}{1+2 \alpha}, \varsigma=\frac{1+\alpha}{1+2 \alpha}$, and $m \geq C a \ln b$, for some appropriate fixed constant $C$, then we satisfy all the conditions, and Lemma 8.1 follows with $\gamma_{D o}=\frac{\alpha \varpi}{8+6 \varpi+(6+4 \varpi) \alpha}$ since the largest term appearing in $(8.50)$ is of order $42 \varepsilon^{\alpha \theta}=b^{-\gamma_{D o} o}$.

\section{Completing the proofs (exponential mixing of $\Phi_{t}$ and Resonances of $X$ )}

9.1. Proofs of Theorem $\mathbf{1 . 2}$ and 1.4. The key step for exponential mixing consists in showing the following consequence of the Lasota-Yorke estimates and the Dolgopyat bound on $\mathcal{R}(z)$ :

Proposition 9.1. For any $0<\gamma<\alpha \leq 1 / 3$, if $\beta>0$ and $1 / q<1$ are such that $\max \{\beta, 1-1 / q\}$ is small enough, then there exist $1 \leq a_{0} \leq a_{1} \leq b_{0}, 0<c_{1}<c_{2}$, and $v_{D o}>0$, so that

$$
\left\|\mathcal{R}(a+i b)^{n}\right\|_{\mathcal{B}} \leq\left(\frac{1}{a+v_{D o}}\right)^{n}
$$

$c_{2} \leq\left(\ln \left(1+a^{-1} v_{D o}\right)\right)^{-1}$ and for all $|b| \geq b_{0}, a \in\left[a_{0}, a_{1}\right]$, and $n \in\left[c_{1} \ln |b|, c_{2} \ln |b|\right]$.

Proposition 9.1 will be shown in the next subsection. Together with Corollary 5.4 it gives the following corollary:

Corollary 9.2 (Spectral gap for the generator $X$ ). Under the assumptions of Corollary 5.2 and Proposition 9.1, there exists $v_{0}>0$ so that the spectrum of $X$ on $\mathcal{B}$ in the half-plane

$$
\left\{z \in \mathbb{C} \mid \Re(z)>-v_{0}\right\}
$$

consists only of the eigenvalue $z=0$, which has algebraic multiplicity equal to one.

Corollaries 5.4 and 9.2 with Lemma 7.5 Remark 5.3 give claims (a) and (b) of Theorem 1.4

Proof. By Proposition 9.1 and Corollary 5.4 the sets $\left\{z \in \mathbb{C}: \Re(z)>-v_{D o},|\Im(z)|>b_{0}\right\}$ and $\{z \in \mathbb{C}: \Re(z)>0\}$ are included in the resolvent set of $X$, in fact, $z \mapsto \mathcal{R}(z)$ is holomorphic, as a bounded operator on $\mathcal{B}$, on the union of these two sets. (Just like for [BL, Cor. 3.10] or [L2, Cor. 2.13], we apply $\mathcal{R}\left(z+\eta^{-1}\right)=\mathcal{R}(z)\left(1+\eta^{-1} \mathcal{R}(z)\right)^{-1}$. See [Bu, Lemma 4.2] for details.)

On the other hand, Corollary 5.4 implies that in the set $\left\{z \in \mathbb{C}: \Re(z)>-v_{D o},|\Im(z)| \leq b_{0}\right\}$ there can be only finitely many eigenvalues, while the intersection of the spectrum with the imaginary axis consists in a simple eigenvalue at $z=0$. Corollary 9.2 is proved.

Proof of Theorems 1.2 and claim (c) of Theorem [1.4. We will apply results of Butterley [Bu] to obtain exponential decay of correlations for Hölder observables (bypassing the final argument in [BL, Proof of Theorem 1.1]). Proposition 9.1 is [Bu, Assumption 3A], Lemma 4.7 is [Bu, Assumption 1], and Corollary 5.2 is [Bu Assumption 2]. In addition, Lemma 4.6 gives strong continuity on $\mathcal{B}$ (in particular the domain $\operatorname{Dom}(X)$ of $X$ is dense). Thus, [Bu, Theorem 1] (noting also the corrigendum) provides a finite set

$$
\left\{z_{j}\right\}_{j=0}^{N}=\operatorname{sp}(X) \cap\left\{z \in \mathbb{C}:-v_{D o}<\Re(z) \leq 0,|\Im(z)| \leq b_{0}\right\},
$$

a (nontrivial) finite rank projector $\Pi: \mathcal{B} \rightarrow \mathcal{B}$, and an operator-valued function $t \mapsto \mathcal{P}_{t}$, where $\mathcal{P}_{t}$ is bounded on $\mathcal{B}, \Pi \mathcal{P}_{t}=\mathcal{P}_{t} \Pi=0$, and a matrix $\widehat{X} \in L(F, F), F=\Pi(\mathcal{B})$, which has exactly $\left\{z_{j}\right\}$ as eigenvalues and

$$
\mathcal{L}_{t}=\mathcal{P}_{t}+e^{t \widehat{X}} \Pi \text { for all } t \geq 0
$$

and for each $v_{1}<v_{D o}$ there exists $C_{v_{1}}>0$ such that, for all $f$ in $\operatorname{Dom} X \subset \mathcal{B}$,

$$
\left|\mathcal{P}_{t} f\right|_{w} \leq C_{v_{1}} e^{-v_{1} t}\|X f\|_{\mathcal{B}} \text { for all } t \geq 0
$$

\footnotetext{
42 Note that with the above choices if $\alpha=\frac{1}{3}$, then we have the rather small value $\gamma_{D o}=\frac{1}{1222}$. Working more it is certainly possible to obtain a better estimate but it remains unclear what the optimal value is.
} 
Next, by Corollary 9.2 , we know that the only $z_{j}$ on the imaginary axis is $z_{0}=0$, corresponding to the constant fixed point of $\mathcal{L}_{t}$ and to the invariant volume $\Pi_{0} f=\int_{\Omega_{0}} f d m$. This implies claim (c) of Theorem 1.4.

Finally, Theorem 1.2 (and Corollary 1.3) follows from applying (9.1) and (9.2) to $f \in \mathcal{C}^{2}\left(\Omega_{0}\right) \cap$ $\mathcal{C}^{0}(\Omega)$ and $\psi \in \mathcal{C}^{1}\left(\Omega_{0}\right)$, since Lemma 2.11 gives

$$
\left|\int \psi \mathcal{P}_{t} f d m\right| \leq\left|\mathcal{P}_{t} f\right|_{\left(\mathcal{C}^{1}\right)^{*}}|\psi|_{\mathcal{C}^{1}} \leq\left|\mathcal{P}_{t} f\right|_{w}|\psi|_{\mathcal{C}^{1}}
$$

while the proof of Lemma 4.6 gives $\mathcal{C}^{2}\left(\Omega_{0}\right) \cap \mathcal{C}^{0}(\Omega) \subset \operatorname{Dom}(X)$ and $\|X f\|_{\mathcal{B}} \leq C|f|_{\mathcal{C}^{2}\left(\Omega_{0}\right)}$.

9.2. Proof of Proposition 9.1. It only remains to deduce Proposition 9.1 from Lemma 8.1 .

Proof of Proposition 9.1. We shall use several times the trivial observation that if $0<\hat{\eta}<\eta$ are small enough then for any $D>1$ we have

$$
\hat{\eta}<\frac{\eta}{D} \Rightarrow \frac{1}{(1+\eta)^{m}} \leq \frac{1}{(1+\hat{\eta})^{D m}} \quad \forall m \geq 1 .
$$

We introduce an auxiliary norm in order to handle the $43|z|$ factor in (5.7) which is much larger than the $|b|^{-\gamma_{D o}}$ decay from the Dolgopyat Lemma 8.1. For fixed $|b| \geq 1$ and $f \in \mathcal{C}^{1}\left(\Omega_{0}\right)$, set

$$
\|f\|_{*}=\|f\|_{s}+\frac{c_{u}}{|b|}\|f\|_{u}+\frac{1}{|b|}\|f\|_{0} .
$$

We shall prove Proposition 9.1 for the norm $\|\cdot\|_{*}$. We first check that this is sufficient: For $M>L>1$, if $a \in\left[a_{0}, a_{1}\right]$ and $|b| \geq b_{0} \geq 1$, where $a_{0}, a_{1}, b_{0}, 0<c_{1}<c_{2}$, and $v_{D o}$ are as in Proposition 9.1 for $\|\cdot\|_{*}$, we have for $c \in\left[c_{1}, c_{2}\right]$ and $m=[c \ln b]$,

$$
\begin{aligned}
\left\|\mathcal{R}^{L m+M m}(a+i b) f\right\|_{\mathcal{B}} & \leq|b|\left\|\mathcal{R}^{L m+M m}(a+i b) f\right\|_{*} \\
& \leq C a^{-L m}\left\|\mathcal{R}^{M m}(a+i b) f\right\|_{*} \\
& \leq C a^{-L m-M m}\left(1+a^{-1} v_{D o}\right)^{-M m}\|f\|_{\mathcal{B}},
\end{aligned}
$$

where we used in the first inequality the fact that $\left.\|\cdot\|_{\mathcal{B}} \leq|b|\|\cdot\|_{*}\right)$, in the second inequality Proposition 9.1 with the bound $|b|\left(1+a^{-1} v_{D o}\right)^{-L m} \leq 1$ for $L$ large enough, and in the last inequality Proposition 9.1 with $\|\cdot\|_{*} \leq\|\cdot\|_{\mathcal{B}}$. Taking large enough $M / L$ and small enough $0<\hat{v}_{D o}<v_{D o}$ (recalling (9.3)), we have proved

$$
\left\|\mathcal{R}^{n}(a+i b)\right\|_{\mathcal{B}} \leq\left(a+\hat{v}_{D o}\right)^{-n}
$$

for some $\hat{v}_{D o}>0$ and $\hat{c}_{1}<\hat{c}_{2}$, all $|b| \geq b_{0}, a \in\left[a_{0}, a_{1}\right]$, any $\hat{c}_{1}<\hat{c}<\hat{c}_{2}$, and $n=[\hat{c} \ln b]$.

Now we prove Proposition 9.1 for the norm $\|\cdot\|_{*}$. Let $f \in \mathcal{B} \cap \mathcal{C}_{\sim}^{0} \cap \mathcal{C}^{2}\left(\Omega_{0}\right)$. We shall assume that $1 \leq a_{0} \leq a_{1} \leq b_{0}$, to be determined later, and take $b \geq b_{0}$ (the case $b \leq-b_{0}$ is similar) and $a \in\left[a_{0}, a_{1}\right]$. In particular

$$
\frac{|a+i b|}{a|b|} \leq 2 .
$$

We shall consider $n=2 m$, the case $n=2 m+1$ is similar. Our starting point is the LasotaYorke estimate (5.8) in the proof of Corollary 5.2, which holds also when replacing $\|\cdot\|_{\mathcal{B}}$ by $\|\cdot\|_{*}$ : Let $0<\tilde{\lambda}<1$ be as in Proposition [5.1, then there exist $44 m_{0} \geq 1$ so that for all $a \geq 1$, all $|b|>0$, and all $m \geq m_{0}$, we have

$$
\left\|\mathcal{R}(a+i b)^{2 m} f\right\|_{*} \leq a^{-m} \nu_{a}^{m}\left\|\mathcal{R}(a+i b)^{m} f\right\|_{*}+C a^{1-m}\left|\mathcal{R}(a+i b)^{m} f\right|_{w}^{\mathbb{H}},
$$

\footnotetext{
43 In GLP another approach was used to address this issue, involving an auxiliary norm $\|\cdot\|^{*}$ based on exact stable leaves (bypassing the neutral norm, using the resolvent). See GLP, Lemma 7.4, Lemma 7.8, just after (7.4)].

${ }^{44}$ We use here that the constant $C$ in (5.8) is uniform in $a$ and $b$, also for $\|\cdot\|_{*}$.
} 
where $\nu_{a}^{m} \leq C\left(1-a^{-1} \ln \tilde{\lambda}\right)^{-m}<1$, and where the homogeneous weak norm $|f| \underset{w}{\mathbb{H}}$ was defined in (7.8). (Indeed, by Definition 3.1 of the partition $\mathcal{G}_{t}(W)$, all the admissible stable curves appearing in the right-hand sides of Propositions 4.1 and 5.1 are homogeneous.)

Recalling (9.3), take $v_{D o}>0$ so that

$$
0<v_{D o}<-\ln \tilde{\lambda} / 2 .
$$

Since (5.8) for $\|\cdot\|_{*}$ also implies

$$
a^{-m} \nu_{a}^{m}\left\|\mathcal{R}^{m} f\right\|_{*} \leq C_{\#} a^{-2 m} \nu_{a}^{m}\|f\|_{*} \leq C_{\#} a^{-2 m} \frac{1}{\left(1-a^{-1} \ln \tilde{\lambda}\right)^{m}}\|f\|_{*},
$$

and since we assume $a \geq a_{0} \geq 1$ the first term in the right-hand side of (9.6) is bounded by

$$
\frac{a^{-2 m}}{6\left(1+a^{-1} v_{D o}\right)^{2 m}}\|f\|_{*}=\frac{1}{6\left(a+v_{D o}\right)^{2 m}}\|f\|_{*},
$$

taking $D=2$ in (9.3), if $b_{0}$ is large enough and if $c_{1}>0$.

We may thus focus on the second term in the right-hand side of (9.6), that is, the homogeneous weak norm contribution. For $\sigma>1$ to be determined later, we fix

$$
\epsilon=|b|^{-\sigma} .
$$

Then, using (5.4), which gives $\left|\mathcal{R}(z)^{m}\right|_{w} \leq C_{\#} a^{-m}$, we find (recalling that $1 \leq a \leq a_{1}$ )

$$
C_{\#} a^{1-m}\left|\mathcal{R}(z)^{m}(f)\right|_{w}^{\mathbb{H}} \leq C_{\#}\left[a^{-m}\left|\mathcal{R}(z)^{m}\left(\mathbb{M}_{\epsilon}(f)\right)\right|_{w}^{\mathbb{H}}+C_{\#} a^{-2 m}\left|f-\mathbb{M}_{\epsilon}(f)\right|_{w}^{\mathbb{H}}\right] .
$$

If $q>1$ is close enough to 1 and $\beta>0$ is small enough, then $\min \left\{\gamma,(2 q)^{-1}, 1 / q-2 / 5-\beta\right\}=\gamma$, since $\gamma<1 / 3$. By Lemma 7.4, the second term on the right-hand side of (9.7) is thus bounded by

$$
C_{\#} L_{0}^{1 / q} a^{-2 m} \epsilon^{\gamma}\|f\|_{\mathcal{B}} \leq C_{\#} a^{-2 m}|b|^{1-\sigma \gamma}\|f\|_{*}
$$

Next, if

$$
\sigma \gamma>1
$$

we get

$$
C_{\#} a^{-2 m}|b|^{1-\sigma \gamma} \leq \frac{1}{6\left(a+v_{D o}\right)^{2 m}}
$$

if $b \geq b_{0}$ is large enough and if

$$
2 m \leq \frac{(\sigma \gamma-1) \ln |b|-\ln \left(6 C_{\#}\right)}{\ln \left(1+a^{-1} v_{D o}\right)} .
$$

The numerator in the right-hand side above is $>(\sigma \gamma-1) \ln |b| / 2$ for large enough $b_{0}$ and $a_{0} / v_{D o}$, since $a \geq a_{0}$. This gives a constraint satisfied if

$$
c_{2} \leq \frac{a_{0}(\sigma \gamma-1)}{4 v_{D o}}
$$

The first term in (9.7) will be more tricky to handle. Recalling $a \leq a_{1}$, we must estimate:

$$
C_{\#} a^{-m} \sup _{\substack{\psi \in C^{\alpha}(W) \\|\psi|_{C^{\alpha}(W)} \leq 1}} \int_{W} \mathcal{R}(z)^{m}\left(\mathbb{M}_{\epsilon}(f)\right) d m_{W}
$$


on some admissible stable homogeneous curve $W$. Recall $\Lambda=\Lambda_{0}^{1 / \tau_{\max }}$ from (1.2). By the Dolgopyat bound (Lemma 8.1), there exist $C_{D o}>0$ and $\gamma_{D o}$ (independent of $1 / q$ and $\beta$ ), so that, if 45

$$
m \geq C_{D o} \ln |b| \quad \text { i.e., } \quad c_{1} \geq C_{D o},
$$

then (9.11) is bounded by

$$
C_{\#} a^{-2 m}|b|^{-\gamma_{D o}}\left(\left|\mathbb{M}_{\epsilon}(f)\right|_{\infty\left(\Omega_{0}\right)}+\frac{\left|\mathbb{M}_{\epsilon}(f)\right|_{H_{\infty}^{1}\left(\Omega_{0}\right)}}{\left(1+a^{-1} \ln \Lambda\right)^{m / 2}}\right) .
$$

Now, Lemma 7.3 and Lemma 2.11 give

$$
\left|\mathbb{M}_{\epsilon}(f)\right|_{L^{\infty}\left(\Omega_{0}\right)} \leq C_{\#} \epsilon^{-\beta-1+1 / q}\|f\|_{s} \leq C_{\#} \epsilon^{-\beta-1+1 / q}\|f\|_{*}
$$

and

$$
\left|\mathbb{M}_{\epsilon}(f)\right|_{H_{\infty}^{1}\left(\Omega_{0}\right)} \leq C_{\#} \epsilon^{-\beta-2+1 / q}\|f\|_{s} \leq C_{\#} \epsilon^{-\beta-2+1 / q}\|f\|_{*} .
$$

On the one hand, if

$$
\sigma(\beta+1-1 / q)<\gamma_{D o},
$$

the estimate (9.14) gives the following bound for the first term of (9.13)

$$
C_{\#} a^{-2 m}|b|^{-\gamma_{D o}+\sigma(\beta+1-1 / q)}\|f\|_{*} \leq \frac{1}{6\left(a+v_{D o}\right)^{2 m}}\|f\|_{*},
$$

if $b_{0}$ is large enough, and

$$
2 m \leq \frac{\left(\gamma_{D o}-\sigma(\beta+1-1 / q)\right) \ln |b|-\ln \left(6 C_{\#}\right)}{\ln \left(1+a^{-1} v_{D o}\right)} .
$$

which holds if $b_{0}$ is large enough and

$$
c_{2} \leq a_{0} \frac{\gamma_{D o}-\sigma(\beta+1-1 / q)}{2 v_{D o}} .
$$

On the other hand, recalling $1 \leq a_{0} \leq a \leq a_{1}$, (9.15) gives that the second term of (9.13) is bounded by $\|f\|_{*}$ multiplied by (note that (9.9) implies $\sigma(\beta+2-1 / q)>\gamma^{-1}$, while $\gamma^{-1} \geq 3>$ $\left.\gamma_{D o}\right)$

$$
C_{\#} \frac{a^{-2 m}|b|^{-\gamma_{D o}+\sigma(\beta-1 / q+2)}}{\left(1+a^{-1} \ln \Lambda\right)^{m / 2}} \leq C_{\#} \frac{a^{-2 m}|b|^{-\gamma_{D o}+\sigma(\beta-1 / q+2)}}{\left(1+a^{-1} \ln \Lambda\right)^{m / 4}\left(1+a^{-1} \ln \Lambda\right)^{m / 4}} \leq \frac{1}{6\left(1+a^{-1} v_{D o}\right)^{2 m}},
$$

if $b_{0}$ is large enough and $m$ is large enough, more precisely

$$
c_{1}>4 \frac{\sigma(\beta+2-1 / q)-\gamma_{D o}}{\ln \left(1+a^{-1} \ln \Lambda\right)},
$$

and if, in addition, recalling (9.3), we assume $v_{D o}<\frac{\ln \Lambda}{8}$.

Along the way, we have collected the lower bounds on $c_{1}$ given by (9.18) and (9.12), the upper bounds on $c_{2}$ listed in (9.10) and (9.17), as well as the conditions (9.9) (lower bound on $\sigma$ ) and (9.16) (upper bound on $\sigma$ ). Fixing $\alpha=1 / 3$, Lemma 8.1 provides values for $\gamma_{D o}$ and $C_{D o}$. If $b_{0}>1$ is large enough and $v_{D o}>0$ is small enough, then all conditions can be satisfied simultaneously if $1 / q<1$ is close enough to 1 and $\beta>0$ is small enough. In particular, recalling $\sigma^{-1}<\gamma \leq \alpha-\beta$ we require

$$
\beta+1-1 / q<\frac{\gamma_{D o}}{\sigma}<\gamma_{D o}(\alpha-\beta)
$$

\footnotetext{
45 The proof gives a constant $C_{D o}$ much larger than $\gamma_{D o}$, and we may safely assume that $\gamma_{D o}<1$.

46 We may take $\gamma=\alpha-\beta$, which is smaller than (and close to) $1 / 3<1 / q$, given our other choices, so that $\sigma$ is larger than (and close to) 3 .
} 
This completes the proof of Proposition 9.1

\section{Appendix A. Proofs of Lemmas 6.6 and 6.7 (approximate Foliation holonomy)}

This section contains the proofs of Lemmas 6.6 and 6.7 (Subsections 6.2 and 6.4) on the Hölder bounds of the Jacobians of the holonomy of the fake (approximate) foliation. These bounds are instrumental to get the four-point estimate (vii) for the fake unstable foliation.

Proof of Lemma 6.6. Letting $\mathbf{h}_{V_{1}^{n}, V_{2}^{n}}$ denote the holonomy map from $V_{1}^{n}=T^{-n} V_{1}$ to $V_{2}^{n}=$ $T^{-n} V_{2}$, we begin by noting that

$$
\begin{aligned}
& \ln \frac{J \mathbf{h}_{12}\left(v_{1}\left(\bar{x}^{s}\right), \bar{x}^{s}\right)}{J \mathbf{h}_{12}\left(v_{1}\left(\bar{y}^{s}\right), \bar{y}^{s}\right)} \\
& =\ln \frac{J_{V_{1}}^{s} T^{-n}\left(v_{1}\left(\bar{x}^{s}\right), \bar{x}^{s}\right)}{J_{V_{2}}^{s} T^{-n}\left(\mathbf{h}_{12}\left(v_{1}\left(\bar{x}^{s}\right), \bar{x}^{s},\right)\right)}-\ln \frac{J_{V_{1}}^{s} T^{-n}\left(v_{1}\left(\bar{y}^{s}\right), \bar{y}^{s}\right)}{J_{V_{2}}^{s} T^{-n}\left(\mathbf{h}_{12}\left(v_{1}\left(\bar{y}^{s}\right), \bar{y}^{s}\right)\right)}+\ln \frac{J \mathbf{h}_{V_{1}^{n}, V_{2}^{n}}\left(x_{n}\right)}{J \mathbf{h}_{V_{1}^{n}, V_{2}^{n}\left(y_{n}\right)}} \\
& =\sum_{j=0}^{n-1} \ln J_{T^{-j} V_{1}}^{s} T^{-1}\left(x_{j}\right)-\ln J_{T^{-j} V_{2}}^{s} T^{-1}\left(\tilde{x}_{j}\right)-\ln J_{T^{-j} V_{1}}^{s} T^{-1}\left(y_{j}\right)+\ln J_{T^{-j} V_{2}}^{s} T^{-1}\left(\tilde{y}_{j}\right) \\
& \quad+\ln \frac{J \mathbf{h}_{V_{1}^{n}, V_{2}^{n}}\left(x_{n}\right)}{J \mathbf{h}_{V_{1}^{n}, V_{2}^{n}}\left(y_{n}\right)},
\end{aligned}
$$

where as in the proof of Lemma 6.4, $x_{j}=T^{-j}\left(v_{1}\left(\bar{x}^{s}\right), \bar{x}^{s}\right), \tilde{x}_{j}=T^{-j}\left(\mathbf{h}_{12}\left(v_{1}\left(\bar{x}^{s}\right), \bar{x}^{s}\right)\right)$, and similarly for $y_{j}$ and $\tilde{y}_{j}$.

We begin by estimating the difference of stable Jacobians in (A.1). The factors in each term involving the stable Jacobian are given by (6.4), and we can bound the differences by grouping together either the terms on the same stable curve (standard distortion bounds), or the terms on the same unstable curve (using Lemma 6.4). Assuming without loss of generality that $d\left(x_{0}, \tilde{x}_{0}\right) \geq d\left(y_{0}, \tilde{y}_{0}\right)$, this yields the following bound on the sum,

$$
C \sum_{j=0}^{n-1} \min \left\{d\left(x_{j+1}, y_{j+1}\right) k_{j+1}^{2}, d\left(x_{j+1}, \tilde{x}_{j+1}\right) k_{j+1}^{2}+d\left(x_{j}, \tilde{x}_{j}\right)+\phi\left(x_{j}, \tilde{x}_{j}\right)+\phi\left(x_{j+1}, \tilde{x}_{j+1}\right)\right\}
$$

where we have used the uniform bound on $J \mathbf{h}_{12}$ given by Lemma 6.4 and (6.7) to eliminate terms involving $d\left(\tilde{x}_{j}, \tilde{y}_{j}\right)$ and $d\left(y_{j}, \tilde{y}_{j}\right)$. We estimate each term using one of two cases.

Case 1. $d\left(x_{j+1}, y_{j+1}\right) \leq d\left(x_{j+1}, \tilde{x}_{j+1}\right)$. We write,

$$
\begin{aligned}
d\left(x_{j+1}, y_{j+1}\right) & =d\left(x_{j+1}, y_{j+1}\right)^{\varpi} d\left(x_{j+1}, y_{j+1}\right)^{1-\varpi} \\
& \leq C\left(J_{V_{1}}^{s} T^{-j-1}\left(x_{0}\right)\right)^{\varpi} d\left(x_{0}, y_{0}\right)^{\varpi} d\left(x_{j+1}, \tilde{x}_{j+1}\right)^{1-\varpi} .
\end{aligned}
$$

On the one hand, we have

$$
d\left(x_{j+1}, \tilde{x}_{j+1}\right) \leq C d\left(x_{0}, \tilde{x}_{0}\right)\left(J_{T^{n-j} \ell}^{u} T^{j+1}\left(x_{j}\right)\right)^{-1},
$$

where $\ell$ is the curve so that $x_{0} \in T^{n}(\ell)=: \bar{\gamma}$. On the other hand, by the uniform transversality of the curves $T^{-j-1}\left(V_{1}\right)$ and $T^{-j-1}(\bar{\gamma})$, we have

$$
\begin{aligned}
& J_{V_{1}}^{s} T^{-j-1}\left(x_{0}\right) J_{\bar{\gamma}}^{u} T^{-j-1}\left(x_{0}\right)=C^{ \pm 1} J T^{-j-1}\left(x_{0}\right)=C^{ \pm 1} \frac{\cos \varphi\left(x_{0}\right)}{\cos \varphi\left(x_{j+1}\right)} \\
& \Longrightarrow J_{V_{1}}^{s} T^{-j-1}\left(x_{0}\right)=C^{ \pm 1} k_{W}^{-2} k_{j+1}^{2} J_{T^{-j-1}(\bar{\gamma})}^{u} T^{j+1}\left(x_{j+1}\right),
\end{aligned}
$$

where $J T^{-j-1}$ is the full Jacobian of the map $T^{-j-1}$. This estimate together with (6.3) implies

$$
J_{V_{1}}^{s} T^{-j-1}\left(x_{0}\right) \leq C k_{W}^{-10 / 3} \rho^{-2 / 3}\left(J_{T^{-j-1} \bar{\gamma}}^{u} T^{j+1}\left(x_{j+1}\right)\right)^{5 / 3} .
$$


Now using this estimate together with (A.3) and again (6.3) yields,

$$
d\left(x_{j+1}, y_{j+1}\right) k_{j+1}^{2} \leq C d\left(x_{0}, y_{0}\right)^{\varpi} d\left(x_{0}, \tilde{x}_{0}\right)^{1-\varpi} \frac{k_{W}^{-(4+10 \varpi) / 3}}{\rho^{2(1+\varpi) / 3}} \frac{1}{\left(J_{T^{-j-1} \bar{\gamma}}^{u} T^{j+1}\left(x_{j+1}\right)\right)^{(1-8 \varpi) / 3}},
$$

and this will be summable over $j$ as long as $\varpi<1 / 8$.

Case 2. $d\left(x_{j+1}, \tilde{x}_{j+1}\right) \leq d\left(x_{j+1}, y_{j+1}\right)$. In order to control the terms involving $\phi\left(x_{j}, \tilde{x}_{j}\right)$, we use the expressions for the Jacobians given by (6.4) and (A.4) together with [CM, eq. (5.27) and following] to write,

$$
\begin{aligned}
\phi\left(x_{j}, \tilde{x}_{j}\right) & \leq C \sum_{m=1}^{j} \frac{d\left(x_{j-m}, \tilde{x}_{j-m}\right)}{J_{T^{m-j-1} V_{1}}^{s} T^{-m+1}\left(x_{j-m+1}\right) J_{T^{-j} \bar{\gamma}}^{u} T^{m-1}\left(x_{j}\right)}+C \frac{\phi\left(x_{0}, \tilde{x}_{0}\right)}{J_{V_{1}}^{s} T^{-j}\left(x_{0}\right) J_{T^{-j} \bar{\gamma}}^{u} T^{j}\left(x_{j}\right)} \\
& \leq C d\left(x_{0}, \tilde{x}_{0}\right) \sum_{m=1}^{j} \frac{\Lambda_{0}^{-m+1}}{J_{T^{m-j} \bar{\gamma}}^{u} T^{j-m}\left(x_{j-m}\right) J_{T^{-j} \bar{\gamma}}^{u} T^{m-1}\left(x_{j}\right)}+C \frac{\Lambda_{0}^{-j} d\left(x_{0}, \tilde{x}_{0}\right)}{J_{T^{-j} \bar{\gamma}}^{u} T^{j}\left(x_{j}\right)},
\end{aligned}
$$

where in the second line we have used the assumption that $\phi\left(x_{0}, \tilde{x}_{0}\right)$ is proportional to $d\left(x_{0}, \tilde{x}_{0}\right)$ and that $d\left(x_{j-m}, \tilde{x}_{j-m}\right) \leq C d\left(x_{0}, \tilde{x}_{0}\right) / J_{T^{m-j} \bar{\gamma}}^{u} T^{j-m}\left(x_{j-m}\right)$. Note that for terms with $m \geq 2$, there is a gap between the expansion factors of the unstable Jacobians in the denominator of the sum: The Jacobian $J_{T^{m-j-1} \bar{\gamma}}^{u} T\left(x_{j-m+1}\right)$ is missing. We use the fact that this is proportional to $k_{j-m}^{2}$ to estimate,

$$
\begin{aligned}
\frac{d\left(x_{0}, \tilde{x}_{0}\right)^{9 \varpi}}{J_{T^{m-j} \bar{\gamma}}^{u} T^{j-m}\left(x_{j-m}\right) J_{T^{-j} \bar{\gamma}}^{u} T^{m-1}\left(x_{j}\right)} & \leq \frac{C d\left(x_{j}, \tilde{x}_{j}\right)^{9 \varpi} k_{j-m}^{18 \varpi}}{\left(J_{T^{m-j} \bar{\gamma}}^{u} T^{j-m}\left(x_{j-m}\right) J_{T^{-j}}^{u} T^{m-1}\left(x_{j}\right)\right)^{1-9 \varpi}} \\
& \leq \frac{C d\left(x_{j}, \tilde{x}_{j}\right)^{9 \varpi} k_{W}^{-12 \varpi} \rho^{-6 \varpi}}{\left(J_{T^{m-j} \bar{\gamma}}^{u} T^{j-m}\left(x_{j-m}\right)\right)^{1-15 \varpi}\left(J_{T^{-j}}^{u} T^{m-1}\left(x_{j}\right)\right)^{1-9 \varpi}} \\
& \leq C d\left(x_{j}, \tilde{x}_{j}\right)^{9 \varpi} k_{W}^{-12 \varpi} \rho^{-6 \varpi} \Lambda_{0}^{-(j-1)(1-15 \varpi)},
\end{aligned}
$$

where we have used (6.3) in the second line. Notice that this estimate holds for $m=1$ as well. Putting this estimate together with (A.7) yields,

$$
\phi\left(x_{j}, \tilde{x}_{j}\right) \leq C d\left(x_{0}, \tilde{x}_{0}\right)^{1-9 \varpi} d\left(x_{j}, \tilde{x}_{j}\right)^{9 \varpi} k_{W}^{-12 \varpi} \rho^{-6 \varpi} \Lambda_{0}^{-(j-1)(1-15 \varpi)} .
$$

A similar estimate holds for $\phi\left(x_{j+1}, \tilde{x}_{j+1}\right)$ with $d\left(x_{j+1}, \tilde{x}_{j+1}\right)$ in place of $d\left(x_{j}, \tilde{x}_{j}\right)$.

Now since $d\left(x_{j}, \tilde{x}_{j}\right)=C^{ \pm 1} d\left(x_{j+1}, \tilde{x}_{j+1}\right) J_{T^{-j-1}}^{u} T\left(x_{j+1}\right)$, we have

$$
d\left(x_{j}, \tilde{x}_{j}\right) \leq C d\left(x_{j+1}, y_{j+1}\right) k_{j}^{2}
$$

by the assumption of this case as well. Since $d\left(x_{j+1}, \tilde{x}_{j+1}\right) \leq C d\left(x_{j}, \tilde{x}_{j}\right)$, combining similar terms in (A.2), it remains to estimate the following expression in this case,

$$
\begin{aligned}
& d\left(x_{j+1}, \tilde{x}_{j+1}\right) k_{j+1}^{2}+d\left(x_{j}, \tilde{x}_{j}\right)+d\left(x_{0}, \tilde{x}_{0}\right)^{1-9 \varpi} d\left(x_{j}, \tilde{x}_{j}\right)^{9 \varpi} k_{W}^{-12 \varpi} \rho^{-6 \varpi} \Lambda_{0}^{-(j-1)(1-15 \varpi)} \\
& \leq d\left(x_{j+1}, \tilde{x}_{j+1}\right) k_{j+1}^{2}+d\left(x_{j+1}, \tilde{x}_{j+1}\right) k_{j}^{2}+\frac{d\left(x_{0}, \tilde{x}_{0}\right)^{1-9 \varpi} d\left(x_{j+1}, \tilde{x}_{j+1}\right)^{9 \varpi} k_{j}^{18 \varpi}}{k_{W}^{12 \varpi} \rho^{6 \varpi} \Lambda_{0}^{(j-1)(1-15 \varpi)}} .
\end{aligned}
$$

For the first term, we use the assumption of this case to write,

$$
\begin{aligned}
d\left(x_{j+1}, \tilde{x}_{j+1}\right) & =d\left(x_{j+1}, \tilde{x}_{j+1}\right)^{\varpi} d\left(x_{j+1}, \tilde{x}_{j+1}\right)^{1-\varpi} \\
& \leq C\left(J_{V_{1}}^{s} T^{-j-1}\left(x_{0}\right)\right)^{\varpi} d\left(x_{0}, y_{0}\right)^{\varpi} d\left(x_{j+1}, \tilde{x}_{j+1}\right)^{1-\varpi},
\end{aligned}
$$

which is the same expression as in (A.3). Thus the first term in (A.9) is bounded by (A.6). 
For the second term in (A.9), we use (6.3) to bound $k_{j}^{2}$ and again (A.5) to estimate,

$$
\begin{aligned}
d\left(x_{j+1}, \tilde{x}_{j+1}\right) k_{j}^{2} & \leq C\left(J_{V_{1}}^{s} T^{-j-1}\left(x_{0}\right)\right)^{\varpi} d\left(x_{0}, y_{0}\right)^{\varpi} \frac{d\left(x_{0}, \tilde{x}_{0}\right)^{1-\varpi}}{\left(J_{T^{-j-1}}^{u} T^{j+1}\left(x_{j+1}\right)\right)^{1-\varpi}} \frac{\left(J_{T^{-j} \bar{\gamma}}^{u} T^{j}\left(x_{j}\right)\right)^{2 / 3}}{k_{W}^{4 / 3} \rho^{2 / 3}} \\
& \leq C d\left(x_{0}, y_{0}\right)^{\varpi} d\left(x_{0}, \tilde{x}_{0}\right)^{1-\varpi} \frac{k_{W}^{-(4+10 \varpi) / 3}}{\rho^{2(1+\varpi) / 3}} \frac{1}{\left(J_{T^{-j-1}}^{u} T^{j+1}\left(x_{j+1}\right)\right)^{(1-8 \varpi) / 3}},
\end{aligned}
$$

where in the second line we have used the fact that $J_{T^{-j} \bar{\gamma}}^{u} T^{j}\left(x_{j}\right) \leq C J_{T^{-j-1} \bar{\gamma}}^{u} T^{j+1}\left(x_{j+1}\right)$ as well as (A.4) to obtain a bound equivalent to (A.6).

Finally, we estimate the third term in (A.9) following a similar strategy, using again (A.5),

$$
\begin{aligned}
& d\left(x_{j+1}, \tilde{x}_{j+1}\right)^{9 \varpi} k_{j}^{18 \varpi} \\
& \quad \leq C\left(J_{V_{1}}^{s} T^{-j-1}\left(x_{0}\right)\right)^{\varpi} d\left(x_{0}, y_{0}\right)^{\varpi} \frac{d\left(x_{0}, \tilde{x}_{0}\right)^{8 \varpi}}{\left(J_{T^{-j-1} \bar{\gamma}}^{u} T^{j+1}\left(x_{j+1}\right)\right)^{8 \varpi}} \frac{\left(J_{T^{-j}}^{u} T^{j}\left(x_{j}\right)\right)^{6 \varpi}}{k_{W}^{12 \varpi} \rho^{6 \varpi}} \\
& \quad \leq C d\left(x_{0}, y_{0}\right)^{\varpi} d\left(x_{0}, \tilde{x}_{0}\right)^{8 \varpi} \frac{k_{W}^{-46 \varpi / 3}}{\rho^{20 \varpi / 3}} \frac{1}{\left(J_{T^{-j-1} \bar{\gamma}}^{u} T^{j+1}\left(x_{j+1}\right)\right)^{\varpi / 3}} .
\end{aligned}
$$

Putting these three estimates together in (A.9) implies that the minimum factor from (A.2) in this case is bounded by

$$
C d\left(x_{0}, y_{0}\right)^{\varpi} d\left(x_{0}, \tilde{x}_{0}\right)^{1-\varpi} \Lambda_{0}^{-(j-1)(1-44 \varpi / 3)} \max \left\{k_{W}^{-82 \varpi / 3} \rho^{-38 \varpi / 3}, k_{W}^{-4 / 3-10 \varpi / 3} \rho^{-2(1+\varpi) / 3}\right\},
$$

which will be summable over $j$ as long as $\varpi \leq 1 / 15$.

Finally, using Case 1 or Case 2 as appropriate for each term appearing in (A.2) and summing over $j$ completes the required estimate on the difference of stable Jacobians appearing in A.1).

It remains to estimate the term involving $J \mathbf{h}_{V_{1}^{n}, V_{2}^{n}}$, which is the holonomy of the seeding foliation $\left\{\ell_{z}\right\}_{z \in \bar{W}_{i}}$. Since $\left\{\ell_{z}\right\}$ is uniformly $\mathcal{C}^{2}$, we have on the one hand,

$$
\ln \frac{J \mathbf{h}_{V_{1}^{n}, V_{2}^{n}}\left(x_{n}\right)}{J \mathbf{h}_{V_{1}^{n}, V_{2}^{n}}\left(y_{n}\right)} \leq C\left(d\left(x_{n}, \tilde{x}_{n}\right)+\phi\left(x_{n}, \tilde{x}_{n}\right)+d\left(y_{n}, \tilde{y}_{n}\right)+\phi\left(y_{n}, \tilde{y}_{n}\right)\right),
$$

while on the other hand, using the fact that the curvatures of $V_{1}^{n}$ and $V_{2}^{n}$ are uniformly bounded,

$$
\ln \frac{J \mathbf{h}_{V_{1}^{n}, V_{2}^{n}}\left(x_{n}\right)}{J \mathbf{h}_{V_{1}^{n}, V_{2}^{n}}\left(y_{n}\right)} \leq C\left(d\left(x_{n}, y_{n}\right)+d\left(\tilde{x}_{n}, \tilde{y}_{n}\right)\right) .
$$

Now $\ln \frac{J \mathbf{h}_{V_{1}^{n}, V_{2}^{n}}\left(x_{n}\right)}{J \mathbf{h}_{V_{1}^{n}, V_{2}^{n}}\left(y_{n}\right)}$ is bounded by the minimum of these two estimates, which we recognize as a simplified version of the expression (A.2) with $j=n$. Thus this quantity satisfies the same bounds as in Cases 1 and 2 above, completing the proof of Lemma 6.6.

Proof of Lemma 6.7. Let $V_{0}$ be the subcurve of $P^{+}(W)$ corresponding to the gap; in the coordinates $\left(\bar{x}^{u}, \bar{x}^{s}\right), V_{0}$ is the vertical line segment identified by the interval $[a, b]$. The boundary curves of the gap from the surviving foliation on either side as described by $\left\{\left(\bar{G}\left(\bar{x}^{u}, a\right), a\right):\left|\bar{x}^{u}\right| \leq k_{W}^{2} \rho\right\}$ and $\left\{\left(\bar{G}\left(\bar{x}^{u}, b\right), b\right):\left|\bar{x}^{u}\right| \leq k_{W}^{2}\right\}$. Let $x_{a}=(0, a)$ and $y_{b}=(0, b)$. Fix $\bar{x}^{u}$ with $\left|\bar{x}^{u}\right| \leq k_{W}^{2} \rho$ and define $\tilde{x}_{a}=\left(\bar{x}^{u}, \bar{G}\left(\bar{x}^{u}, a\right)\right)$ and $\tilde{y}_{b}=\left(\bar{x}^{u}, \bar{G}\left(\bar{x}^{u}, b\right)\right)$. Let $V_{1}$ denote the stable curve (vertical in the $\left(\bar{x}^{u}, \bar{x}^{s}\right)$ coordinates) connecting $\tilde{x}_{a}$ and $\tilde{y}_{b}$.

Using similar notation to Subsection 6.3, let $j+1$ denote the least integer $j^{\prime} \geq 1$ such that $T^{j^{\prime}}\left(\mathcal{S}_{0}\right)$ intersects $V_{0}$. This implies in particular, that the surviving parts of the foliation immediately on either side of the gap lie in the same homogeneity strip for the first $j$ interates of $T^{-1}$. Define $x_{i}=T^{-i}\left(x_{a}\right), y_{i}=T^{-i}\left(y_{b}\right), \tilde{x}_{i}=T^{-i}\left(\tilde{x}_{a}\right)$ and $\tilde{y}_{i}=T^{-i}\left(\tilde{y}_{b}\right)$, for $i=0,1, \ldots j$. 
The following expressions for the quantities appearing in the slope will be useful,

$$
\bar{G}\left(\bar{x}^{u}, b\right)-\bar{G}\left(\bar{x}^{u}, a\right)=\int_{T^{-j} V_{1}} J_{T^{-j} V_{1}}^{s} T^{j} d m_{W}, \quad b-a=\int_{T^{-j} V_{0}} J_{T^{-j} V_{0}}^{s} T^{j} d m_{W} .
$$

And similarly,

$$
\partial_{\bar{x}^{s}} \bar{G}\left(\bar{x}^{u}, a\right)=\frac{J_{T^{-j} V_{1}}^{s} T^{j}\left(y_{j}\right)}{J_{T^{-j} V_{0}}^{s} T^{j}\left(x_{j}\right)} J \mathbf{h}_{-j}\left(x_{j}\right),
$$

where $\mathbf{h}_{-j}$ is the holonomy map from $T^{-j} V_{0}$ to $T^{-j} V_{1}$ along the surviving foliation on the sides of the gap. Using these expressions, we estimate,

$$
\begin{aligned}
\ln \left|\frac{\bar{G}\left(\bar{x}^{u}, b\right)-\bar{G}\left(\bar{x}^{u}, a\right)}{(b-a) \partial_{\bar{x}^{s}} \bar{G}\left(\bar{x}^{u}, a\right)}\right|= & \ln \frac{\frac{1}{\left|T^{-j} V_{1}\right|} \int_{T^{-j} V_{1}} J_{T^{-j} V_{1}}^{s} T^{j} d m_{W}}{J_{T^{-j} V_{1}}^{s} T^{j}\left(y_{j}\right)}-\ln J \mathbf{h}_{-j}\left(x_{j}\right) \\
& +\ln \frac{\frac{1}{\left|T^{-j} V_{0}\right|} \int_{T^{-j} V_{0}} J_{T^{-j} V_{0}}^{s} T^{j} d m_{W}}{\frac{J^{-j} V_{0}}{T^{j}}\left(x_{j}\right)}+\ln \frac{\left|T^{-j} V_{0}\right|}{\left|T^{-j} V_{1}\right|} .
\end{aligned}
$$

Since $J_{T^{-j} V_{1}}^{s} T^{j}$ is continuous on $T^{-j} V_{1}$, the average value of the function is equal to the function evaluated at some point on $T^{-j} V_{1}$. Thus by standard distortion bounds along stable curves, the first term on the right-hand side of (A.10) is bounded by $C_{d}|a-b|^{1 / 3}$. A similar bound holds for the third term above.

In order to estimate $J \mathbf{h}_{-j}\left(x_{j}\right)$, we note that this is part of the surviving foliation that originated at time $-n$. Applying Lemma 6.4 from time $-j$ to time $-n$, there exists $C>0$ such that

$$
\ln J \mathbf{h}_{-j}\left(x_{j}\right) \leq C\left(d\left(x_{j}, \tilde{x}_{j}\right) \rho^{-2 / 3} k_{W}^{-4 / 3}+\phi\left(x_{j}, \tilde{x}_{j}\right)\right),
$$

where $\phi\left(x_{j}, \tilde{x}_{j}\right)$ represents the angle formed by the tangent vectors to $T^{-j} V_{0}$ and $T^{-j} V_{1}$ at $x_{j}$ and $\tilde{x}_{j}$, respectively.

For the first term above, $d\left(x_{j}, \tilde{x}_{j}\right) \leq C d\left(x_{0}, \tilde{x}_{0}\right) / J_{T^{-j} \bar{\gamma}}^{u} T^{j}\left(x_{j}\right)$, where $\bar{\gamma}$ denotes the element of the surviving foliation containing $x_{a}$. For the second term, we use (A.7) to write,

$$
\phi\left(x_{j}, \tilde{x}_{j}\right) \leq C d\left(x_{0}, \tilde{x}_{0}\right) \sum_{m=1}^{j} \frac{\Lambda_{0}^{-m+1}}{J_{T^{m-j} \bar{\gamma}}^{u} T^{j-m}\left(x_{j-m}\right) J_{T^{-j} \bar{\gamma}}^{u} T^{m-1}\left(x_{j}\right)}+C \frac{\Lambda_{0}^{-j} d\left(x_{0}, \tilde{x}_{0}\right)}{J_{T^{-j} \bar{\gamma}}^{u} T^{j}\left(x_{j}\right)},
$$

where we have used the fact that $\phi\left(x_{0}, \tilde{x}_{0}\right) \leq C d\left(x_{0}, \tilde{x}_{0}\right)$ since both $V_{0}$ and $V_{1}$ are vertical segments and the unstable curves in the surviving foliation have uniformly bounded curvature. As in the proof of Lemma 6.6, there is a gap in the product of unstable Jacobians; the factor $J_{T^{m-j} \bar{\gamma}}^{u} T\left(x_{j-m+1}\right)$ is missing. This is proportional to $k_{j-m}^{2}$, but due to (6.3), we do not ask for a full power of this factor; rather, we estimate,

$$
\begin{aligned}
\frac{1}{J_{T^{m-j} \bar{\gamma}}^{u} T^{j-m}\left(x_{j-m}\right) J_{T^{-j} \bar{\gamma}}^{u} T^{m-1}\left(x_{j}\right)} & \leq \frac{C k_{j-m}^{6 / 5}}{J_{T^{m-j} \bar{\gamma}}^{u} T^{j-m}\left(x_{j-m}\right)\left(J_{T^{m-j} \bar{\gamma}}^{u} T\left(x_{j-m+1}\right)\right)^{3 / 5} J_{T^{-j} \bar{\gamma}}^{u} T^{m-1}\left(x_{j}\right)} \\
& \leq C \frac{k_{W}^{4 / 5} \rho^{-2 / 5}}{\left(J_{T^{-j} \bar{\gamma}}^{u} T^{j}\left(x_{j}\right)\right)^{3 / 5}} .
\end{aligned}
$$

This, together with the above estimates implies,

$$
\ln J \mathbf{h}_{-j}\left(x_{j}\right) \leq C\left(\frac{d\left(x_{0}, \tilde{x}_{0}\right)}{\rho^{2 / 3} k_{W}^{4 / 3} J_{T^{-j}}^{u} T^{j}\left(x_{j}\right)}+\frac{d\left(x_{0}, \tilde{x}_{0}\right) k_{W}^{4 / 5}}{\rho^{2 / 5}\left(J_{T^{-j}}^{u} T^{j}\left(x_{j}\right)\right)^{3 / 5}}\right) .
$$


Sublemma A.1 (Relating the gap size and the unstable Jacobian). Let $a, b$ define the gap in $P^{+}(W)$ as in the statement of the lemma and let $j, x_{j}$ be as defined above. There exists $C>0$, depending only on $T$, and not on $n, j$ or $W$, such that

$$
\frac{1}{J_{T^{-j} \bar{\gamma}}^{u} T^{j}\left(x_{j}\right)} \leq C k_{W}^{-2} \rho^{-26 / 35}|a-b|^{9 / 35} .
$$

Proof. We consider the size of the gap created in a neighborhood of $T^{-j-1}\left(x_{a}\right)$, depending on whether this gap is created by an intersection with homogeneity strips of high index or not.

Case 1. The connected component of $T^{-j-1}\left(V_{1}\right)$ containing $T^{-j-1}\left(x_{a}\right)$ intersects $\mathcal{S}_{0}$. Then using (6.12) and (6.13), we have,

$$
|a-b| \geq \frac{C^{ \pm 1} k_{j+1}^{-4}}{J_{V_{1}}^{s} T^{-j}\left(x_{a}\right)} \geq \frac{C^{ \pm 1} k_{W}^{8 / 3} \rho^{4 / 3}}{\left(J_{T^{-j-1}}^{u} T^{j+1}\left(x_{j+1}\right)\right)^{4 / 3} J_{V_{1}}^{s} T^{-j}\left(x_{a}\right)},
$$

where we used (6.3) in the second inequality. Now $J_{T^{-j-1}}^{u} T^{j+1}\left(x_{j+1}\right)=C^{ \pm 1} k_{j}^{2} J_{T^{-j}}^{u} T^{j}\left(x_{j}\right)$. Using this together with (A.4) to convert between stable and unstable Jacobians yields,

$$
|a-b| \geq \frac{C^{ \pm 1} k_{W}^{8 / 3} \rho^{4 / 3}}{\left(J_{T^{-j}}^{u} T^{j}\left(x_{j}\right)\right)^{7 / 3} k_{j}^{14 / 3} k_{W}^{-2}} \geq \frac{C^{ \pm 1} k_{W}^{70 / 9} \rho^{26 / 9}}{\left(J_{T^{-j}}^{u} T^{j}\left(x_{j}\right)\right)^{35 / 9}},
$$

where in the second inequality we have used (6.3). This proves the sublemma in this case.

Case 2. The component of $T^{-j-1}\left(V_{1}\right)$ containing $T^{-j-1}\left(x_{a}\right)$ does not intersect $\mathcal{S}_{0}$. In this case, by the uniform transversality of $\mathcal{S}_{1}$ with unstable curves, the gap is bounded below by

$$
\begin{aligned}
|a-b| & \geq \frac{C^{ \pm 1} \rho k_{W}^{2}}{J_{T^{-j-1} \bar{\gamma}}^{u} T^{j+1}\left(x_{j+1}\right) J_{V_{1}}^{s} T^{-j-1}\left(x_{a}\right)} \geq \frac{C^{ \pm 1} \rho k_{W}^{4}}{\left(J_{T^{-j-1}}^{u} T^{j+1}\left(x_{j+1}\right)\right)^{2} k_{j+1}^{2}} \\
& \geq \frac{C^{ \pm 1} \rho k_{W}^{4}}{\left(J_{T^{-j} \bar{\gamma}}^{u} T^{j}\left(x_{j}\right)\right)^{2}},
\end{aligned}
$$

where in the last step, we have used the fact that in Case $2, k_{j+1}$ is of order 1 . This is clearly a greater lower bound on $|a-b|$ than in Case 1, proving the sublemma.

With the sublemma proved, we return to our estimate (A.11),

$$
\begin{aligned}
\ln J \mathbf{h}_{-j}\left(x_{j}\right) & \leq C\left(\frac{d\left(x_{0}, \tilde{x}_{0}\right)}{\rho^{2 / 3} k_{W}^{4 / 3} J_{T^{-j}}^{u} T^{j}\left(x_{j}\right)}+\frac{d\left(x_{0}, \tilde{x}_{0}\right) k_{W}^{4 / 5}}{\rho^{2 / 5}\left(J_{T^{-j}}^{u} T_{\bar{\gamma}}^{j}\left(x_{j}\right)\right)^{3 / 5}}\right) \\
& \leq C d\left(x_{0}, \tilde{x}_{0}\right)\left(k_{W}^{-10 / 3} \rho^{-148 / 105}|a-b|^{9 / 35}+k_{W}^{-2 / 5} \rho^{-148 / 175}|a-b|^{27 / 175}\right) \\
& \leq C\left(k_{W}^{-4 / 3} \rho^{-43 / 105}|a-b|^{9 / 35}+k_{W}^{8 / 5} \rho^{27 / 175}|a-b|^{27 / 175}\right) \\
& \leq C\left(\rho^{-43 / 105}|a-b|^{9 / 35}+\rho^{-29 / 175}|a-b|^{27 / 175}\right) \leq C \rho^{-31 / 105}|a-b|^{1 / 7},
\end{aligned}
$$

where in the third line we have used the fact that $d\left(x_{0}, \tilde{x}_{0}\right) \leq C \rho k_{W}^{2}$ and in the last line that $k_{W} \leq C \rho^{-1 / 5}$. We have also opted to take simpler (slightly less than optimal) exponents, taking the power $1 / 7$ rather than $27 / 175$ in the second term of the last line and converting $|a-b|^{4 / 35} \leq C \rho^{4 / 35}$ in the first term. Note that $|a-b| \leq C \rho$ follows from Subsection 6.1.

Finally, for the fourth term on the right-hand side of (A.10), we note that the boundary curves for the gap containing $T^{-j} V_{0}$ and $T^{-j} V_{1}$ both lie in the unstable cone. Since $T^{-j} V_{0}$ and $T^{-j} V_{1}$ 
have bounded curvature, we have

$$
\ln \frac{\left|T^{-j} V_{0}\right|}{\left|T^{-j} V_{1}\right|} \leq C d\left(x_{j}, \tilde{x}_{j}\right) \leq \frac{C d\left(x_{0}, \tilde{x}_{0}\right)}{J_{T^{-j}}^{u} T^{j}\left(x_{j}\right)} .
$$

Using Sublemma A.1, this quantity is bounded by

$$
C d\left(x_{0}, \tilde{x}_{0}\right) k_{W}^{-2} \rho^{-26 / 35}|a-b|^{9 / 35} \leq C \rho^{9 / 35}|a-b|^{9 / 35},
$$

where again we have used the fact that $d\left(x_{0}, \tilde{x}_{0}\right) \leq k_{W}^{2} \rho$. Using the estimates for these four terms in (A.10) ends the proof of Lemma 6.7 since both the average slope and $\partial_{\bar{x}} \bar{G}$ are uniformly bounded away from 0 and infinity.

\section{Appendix B. Estimates for the Dolgopyat bound (Lemmas 8.7, 8.8, and 8.9)}

This appendix contains several crucial, but technical, estimates used in the Dolgopyat type cancellation argument developed in Section 8 ,

Proof of Lemma 8.7. Recalling condition (ii) on the foliation from Section 6, the first identity in (8.35) together with (8.34) gives

$$
\begin{aligned}
x^{s} & =G_{i, j, \varkappa}\left(M_{A}\left(x^{s}\right), 0\right)+\int_{0}^{\mathbf{h}_{A}^{s}\left(x^{s}\right)} d s \partial_{x^{s}} G_{i, j, \varkappa}\left(M_{A}\left(x^{s}\right), s\right) \\
& =\int_{0}^{M_{A}\left(x^{s}\right)} d u \partial_{x^{u}} G_{i, j, \varkappa}(u, 0)+\int_{0}^{\mathbf{h}_{A}^{s}\left(x^{s}\right)} d s\left[1+\int_{0}^{M_{A}\left(x^{s}\right)} d u \partial_{x^{u}} \partial_{x^{s}} G_{i, j, \varkappa}(u, s)\right] \\
& =\mathbf{h}_{A}^{s}\left(x^{s}\right)+\int_{0}^{M_{A}\left(x^{s}\right)} d u \partial_{x^{u}} G_{i, j, \varkappa}(u, 0)+\int_{0}^{\mathbf{h}_{A}^{s}\left(x^{s}\right)} d s \int_{0}^{M_{A}\left(x^{s}\right)} d u \partial_{x^{u}} \partial_{x^{s}} G_{i, j, \varkappa}(u, s),
\end{aligned}
$$

where we have used the fact that $\partial_{x^{s}} G_{i, j, \varkappa}(0, s)=1$ for each $s$ by property (ii) of the foliation. Thus, combining the bound (vi) from Section [6] that is $\left|\partial_{x^{u}} \partial_{x^{s}} G_{i, j, \varkappa}\right| \leq C \rho^{-4 / 5}=C \varepsilon^{-4 \varsigma / 5}$, the bound $\left|M_{A}^{\prime}\right| \leq C_{\#} \varepsilon^{1-\frac{4}{5} \theta}$ from (18.22) (which implies $\left|M_{A}\left(x^{s}\right)\right| \leq C_{\#} \varepsilon$ ), and the condition $\left|\mathbf{h}_{A}^{s}\left(x^{s}\right)\right| \leq C_{\#} \varepsilon^{\theta}$ from (8.34), we get $\mathbf{h}_{A}^{s}\left(x^{s}\right)=x^{s}\left(1+\mathcal{O}\left(\varepsilon^{1-4 \varsigma / 5}\right)\right)+\mathcal{O}(\varepsilon)$.

Next, differentiating the first identity in (8.35), we find

$$
\left(\mathbf{h}_{A}^{s}\right)^{\prime}\left(x^{s}\right)=\frac{1-\partial_{x^{u}} G_{i, j, \varkappa}\left(M_{A}\left(x^{s}\right), \mathbf{h}_{A}^{s}\left(x^{s}\right)\right) M_{A}^{\prime}\left(x^{s}\right)}{\partial_{x^{s}} G_{i, j, \varkappa}\left(M_{A}\left(x^{s}\right), \mathbf{h}_{A}^{s}\left(x^{s}\right)\right)} .
$$

We have

$$
\begin{aligned}
\partial_{x^{s}} G_{i, j, \varkappa}\left(M_{A}\left(x^{s}\right), \mathbf{h}_{A}^{s}\left(x^{s}\right)\right) & =\partial_{x^{s}} G_{i, j, \varkappa}\left(0, \mathbf{h}_{A}^{s}\left(x^{s}\right)\right)+\int_{0}^{M_{A}\left(x^{s}\right)} \partial_{x^{u}} \partial_{x^{s}} G_{i, j, \varkappa}\left(u, \mathbf{h}_{A}^{s}\left(x^{s}\right)\right) d u \\
& =1+\mathcal{O}\left(\varepsilon^{1-4 \varsigma / 5}\right),
\end{aligned}
$$

while $\left|\partial_{x^{u}} G_{i, j, \varkappa}\right|_{\infty} \leq C_{\#}$, hence we have the second inequality of Lemma 8.7 .

$$
\left|1-\left(\mathbf{h}_{A}^{s}\right)^{\prime}\right| \leq C_{\#} \varepsilon^{1-\frac{4}{5} \varsigma},
$$

while the bound on $\left|\left(\mathbf{h}_{A}^{s}\right)\left(x^{s}\right)-x^{s}\right|$ follows by integration. Finally, $\mathbf{h}_{A}^{s}$ is invertible and the claimed bound on $\left|\left(\mathbf{h}_{A}^{s}\right)^{-1}\left(x^{s}\right)-x^{s}\right|$ holds using $\left(\left(\mathbf{h}_{A}^{s}\right)^{-1}\left(x^{s}\right)-x^{s}\right)^{\prime}=\frac{1}{\left(\mathbf{h}_{A}^{s}\right)^{\prime} \circ\left(\mathbf{h}_{A}^{s}\right)^{-1}}-1$ and integrating as before.

Proof of Lemma 8.8. To start with, note that formula [BL, (E.1)] is obtained by a purely geometric argument and uses only that the strong manifolds are in the kernel of the contact form 
and the weak manifolds in the kernel of its differential. Since the exact same situation holds here, we have, for all relevant manifolds $W_{A}$, the formula

$$
\boldsymbol{\omega}_{A}\left(x^{s}\right)=\int_{0}^{x^{s}} d s \int_{0}^{M_{A}\left(\left(\mathbf{h}_{A}^{s}\right)^{-1}(s)\right)} d u \partial_{x^{s}} G_{i, j \varkappa}(u, s) .
$$

Note that this implies

$$
\left|\boldsymbol{\omega}_{A}\right|_{\infty} \leq C_{\#} \varepsilon^{1+\theta} .
$$

To obtain the formula we are interested in for each pair $A, B$, it suffices to differentiate. Remembering properties (ii) and (vi) of the foliation constructed in Section [6, we have

$$
\begin{aligned}
\partial_{x^{s}} \boldsymbol{\omega}_{B}\left(x^{s}\right)-\partial_{x^{s}} \boldsymbol{\omega}_{A}\left(x^{s}\right) & =\int_{M_{A}\left(\left(\mathbf{h}_{A}^{s}\right)^{-1}\left(x^{s}\right)\right)}^{M_{B}\left(\left(\mathbf{h}_{B}^{s}\right)^{-1}\left(x^{s}\right)\right)} d u \partial_{x^{s}} G_{i, j, \varkappa}\left(u, x^{s}\right) \\
& =\int_{M_{A}\left(\left(\mathbf{h}_{A}^{s}\right)^{-1}\left(x^{s}\right)\right)}^{M_{B}\left(\left(\mathbf{h}_{B}^{s}\right)^{-1}\left(x^{s}\right)\right)} d u\left[1+\int_{0}^{u} d u_{1} \partial_{x^{u}} \partial_{x^{s}} G_{i, j, \varkappa}\left(u_{1}, x^{s}\right)\right] \\
& =\left[M_{B}\left(\left(\mathbf{h}_{B}^{s}\right)^{-1}\left(x^{s}\right)\right)-M_{A}\left(\left(\mathbf{h}_{A}^{s}\right)^{-1}\left(x^{s}\right)\right)\right]\left(1+\mathcal{O}\left(\varepsilon^{1-\frac{4}{5} \varsigma}\right)\right) .
\end{aligned}
$$

Next, the distance between $W_{A}^{0} \cap\left\{x^{s}=0\right\}$ and $W_{B}^{0} \cap\left\{x^{s}=0\right\}$ is given by $\left|M_{A}(0)-M_{B}(0)\right|=$ : $d\left(W_{A}, W_{B}\right)$. Then, by the argument developed in (8.18) (proof of Lemma 8.5), we have

$$
|| M_{A}(0)-M_{B}(0)|-| M_{A}\left(x^{s}\right)-M_{B}\left(x^{s}\right)|| \leq C_{\#} d\left(W_{A}, W_{B}\right) \varepsilon^{\theta / 5} .
$$

On the other hand, by (B.1) and property (vi) of the foliation, we have

$$
\begin{aligned}
\left|\left(\mathbf{h}_{B}^{s}\right)^{-1}\left(x^{s}\right)-\left(\mathbf{h}_{A}^{s}\right)^{-1}\left(x^{s}\right)\right|= & \left|\int_{M_{A} \circ\left(\mathbf{h}_{A}^{s}\right)^{-1}\left(x^{s}\right)}^{M_{B} \circ\left(\mathbf{h}_{B}^{s}\right)^{-1}\left(x^{s}\right)} d u \partial_{x^{u}} G_{i, j, \varkappa}(u, 0)\right| \\
& +\left|\int_{M_{A} \circ\left(\mathbf{h}_{A}^{s}\right)^{-1}\left(x^{s}\right)}^{M_{B} \circ\left(\mathbf{h}_{B}^{s}\right)^{-1}\left(x^{s}\right)} d u \int_{0}^{x^{s}} d s \partial_{x^{u}} \partial_{x^{s}} G_{i, j, \varkappa}(u, s)\right| \\
\leq & C_{\#}\left(1+\varepsilon^{\theta-\frac{4}{5} \varsigma}\right)\left|M_{B} \circ\left(\mathbf{h}_{B}^{s}\right)^{-1}\left(x^{s}\right)-M_{A} \circ\left(\mathbf{h}_{A}^{s}\right)^{-1}\left(x^{s}\right)\right| .
\end{aligned}
$$

To conclude recall that we are working in coordinates in which $\left|M_{A}^{\prime}\right| \leq C_{\#} \varepsilon^{1-\frac{4}{5}} \theta$, cf. the proof of Lemma 8.5, hence

$$
\begin{aligned}
& \left|M_{B} \circ\left(\mathbf{h}_{B}^{s}\right)^{-1}-M_{A} \circ\left(\mathbf{h}_{A}^{s}\right)^{-1}\right| \geq\left|M_{B} \circ\left(\mathbf{h}_{B}^{s}\right)^{-1}-M_{A} \circ\left(\mathbf{h}_{B}^{s}\right)^{-1}\right|-C_{\#} \varepsilon^{1-\frac{4}{5} \theta}\left|\left(\mathbf{h}_{B}^{s}\right)^{-1}-\left(\mathbf{h}_{A}^{s}\right)^{-1}\right| \\
& \quad \geq\left|M_{B} \circ\left(\mathbf{h}_{B}^{s}\right)^{-1}-M_{A} \circ\left(\mathbf{h}_{B}^{s}\right)^{-1}\right|-C_{\#}\left(\varepsilon^{1-\frac{4}{5} \theta}+\varepsilon^{1-\frac{4}{5} \varsigma+\frac{1}{5} \theta}\right)\left|M_{B} \circ\left(\mathbf{h}_{B}^{s}\right)^{-1}-M_{A} \circ\left(\mathbf{h}_{A}^{s}\right)^{-1}\right|,
\end{aligned}
$$

which, together with (B.6) and (B.7), proves (8.41).

To prove the second statement, let us introduce the shorthand notation $\boldsymbol{\omega}_{A, B}\left(x^{s}\right)=\boldsymbol{\omega}_{A}\left(x^{s}\right)-$ $\boldsymbol{\omega}_{B}\left(x^{s}\right)$ and $\boldsymbol{\eta}_{A}=\left(\mathbf{h}_{A}^{s}\right)^{-1}, \boldsymbol{\eta}_{B}=\left(\mathbf{h}_{B}^{s}\right)^{-1}$. By (B.6) we have

$$
\begin{gathered}
\left|\partial_{x^{s}} \boldsymbol{\omega}_{A, B}\left(x^{s}\right)-\partial_{x^{s}} \boldsymbol{\omega}_{A, B}\left(y^{s}\right)\right|=\left|\int_{M_{A}\left(\boldsymbol{\eta}_{A}\left(x^{s}\right)\right)}^{M_{B}\left(\boldsymbol{\eta}_{B}\left(x^{s}\right)\right)} d u \partial_{x^{s}} G_{i, j, \varkappa}\left(u, x^{s}\right)-\int_{M_{A}\left(\boldsymbol{\eta}_{A}\left(y^{s}\right)\right)}^{M_{B}\left(\boldsymbol{\eta}_{B}\left(y^{s}\right)\right)} d u \partial_{x^{s}} G_{i, j, \varkappa}\left(u, y^{s}\right)\right| \\
\leq\left|\int_{M_{A}\left(\boldsymbol{\eta}_{A}\left(x^{s}\right)\right)}^{M_{B}\left(\boldsymbol{\eta}_{B}\left(x^{s}\right)\right)} d u \partial_{x^{s}} G_{i, j, \varkappa}\left(u, x^{s}\right)-\partial_{x^{s}} G_{i, j, \varkappa}\left(u, y^{s}\right)\right| \\
\quad+\left|\int_{M_{B}\left(\boldsymbol{\eta}_{B}\left(y^{s}\right)\right)}^{M_{B}\left(\boldsymbol{\eta}_{B}\left(x^{s}\right)\right)} d u \partial_{x^{s}} G_{i, j, \varkappa}\left(u, y^{s}\right)\right|+\left|\int_{M_{A}\left(\boldsymbol{\eta}_{A}\left(x^{s}\right)\right)}^{M_{A}\left(\boldsymbol{\eta}_{B}\left(y^{s}\right)\right)} d u \partial_{x^{s}} G_{i, j, \varkappa}\left(u, y^{s}\right)\right|
\end{gathered}
$$


Notice that (ii) of the foliation implies

$$
\begin{gathered}
\partial_{x^{s}} G_{i, j, \varkappa}\left(u, x^{s}\right)-\partial_{x^{s}} G_{i, j, \varkappa}\left(u, y^{s}\right)=\partial_{x^{s}} G_{i, j, \varkappa}\left(u, x^{s}\right)-\partial_{x^{s}} G_{i, j, \varkappa}\left(u, y^{s}\right)-\partial_{x^{s}} G_{i, j, \varkappa}\left(0, x^{s}\right) \\
+\partial_{x^{s}} G_{i, j, \varkappa}\left(0, y^{s}\right) .
\end{gathered}
$$

Thus, the four-point property (vii) (applied to the points $\left(u, x^{s}\right)$ and $\left.\left(0, y^{s}\right)\right)$ implies

$$
\left|\partial_{x^{s}} G_{i, j, \varkappa}\left(u, x^{s}\right)-\partial_{x^{s}} G_{i, j, \varkappa}\left(u, y^{s}\right)\right| \leq C_{\#} \varepsilon^{-\left(\frac{4}{5}+\frac{11 \varpi}{15}\right) \varsigma+1-7 \varpi}\left|x^{s}-y^{s}\right|^{\varpi} .
$$

On the other hand, equation (8.22) and Lemma 8.7 imply

$$
\left|M_{B}\left(\boldsymbol{\eta}_{B}\left(x^{s}\right)\right)-M_{B}\left(\boldsymbol{\eta}_{B}\left(y^{s}\right)\right)\right| \leq C_{\#} \varepsilon^{1-\frac{4}{5} \theta}\left|x^{s}-y^{s}\right|,
$$

and the same for $A$. Remembering that $\left|x^{s}\right|,\left|y^{s}\right| \leq c \varepsilon^{\theta}$, property (v) of the foliation, and our conditions on $\varpi, \theta$, from (8.42), the above facts yield

$$
\left|\partial_{x^{s}} \boldsymbol{\omega}_{A, B}\left(x^{s}\right)-\partial_{x^{s}} \boldsymbol{\omega}_{A, B}\left(y^{s}\right)\right| \leq C_{\#} \varepsilon\left|x^{s}-y^{s}\right|^{\varpi}+\varepsilon^{1-\frac{4}{5} \theta}\left|x^{s}-y^{s}\right| \leq C_{\#} \varepsilon\left|x^{s}-y^{s}\right|^{\varpi}
$$

which, together with (B.5), proves the second statement of Lemma 8.8 .

To continue, let

$$
\boldsymbol{L}_{A, B}\left(x^{s}, x^{0}\right)=\frac{[(m-1) !]^{2}}{\left(\ell \tau_{-}\right)^{2 m-2}} e^{-2 a \ell \tau_{-}} \mathbf{G}_{\ell, m, i, A}^{*}\left(x^{s}, x^{0}\right) \overline{\mathbf{G}_{\ell, m, i, B}^{*}\left(x^{s}, x^{0}\right)} .
$$

Next we introduce a sequence $\left\{w_{j}\right\}_{j=0}^{M} \subset \mathbb{R}$ such that $w_{0}=-c r^{\theta}$ and $\partial_{x^{s}} \boldsymbol{\omega}_{A, B}\left(w_{j}\right)\left(w_{j+1}-w_{j}\right)=$ $2 \pi b^{-1}$ and let $M \in \mathbb{N}$ be such that $w_{M} \leq c \varepsilon^{\theta}$ and $w_{M+1}>c \varepsilon^{\theta} 47$ Also, we set $\boldsymbol{\delta}_{j}=w_{j+1}-w_{j}$. By Lemma 8.7 it follows that, for each $x^{s} \in\left[w_{j}, w_{j+1}\right]$,

$$
\left|\boldsymbol{\omega}_{A, B}\left(x^{s}\right)-\boldsymbol{\omega}_{A, B}\left(w_{j}\right)-\partial_{x^{s}} \boldsymbol{\omega}_{A, B}\left(w_{j}\right)\left(x^{s}-w_{j}\right)\right| \leq C_{\# \varepsilon} \boldsymbol{\delta}_{j}^{1+\varpi} .
$$

In addition, the bounds in (8.39) imply

$$
\left|\boldsymbol{L}_{A, B}\left(x^{s}, x^{0}\right)-\boldsymbol{L}_{A, B}\left(w_{j}, x^{0}\right)\right| \leq C_{\#} \boldsymbol{\delta}_{j} \varepsilon^{-3 \theta} .
$$

Then, using the first part of the Lemma,

$$
\begin{aligned}
& \left|\int_{w_{j}}^{w_{j+1}} e^{-i b \boldsymbol{\omega}_{A, B}\left(x^{s}\right)} \boldsymbol{L}_{A, B}\left(x^{s}, x^{0}\right) d x^{s}\right| \\
& \quad=\left|\int_{w_{j}}^{w_{j+1}} e^{-i b\left[\partial_{x} s \boldsymbol{\omega}_{A, B}\left(w_{i}\right)\left(x^{s}-w_{j}\right)+\mathcal{O}\left(\varepsilon \boldsymbol{\delta}_{j}^{1+\varpi}\right)\right]}\left[\boldsymbol{L}_{A, B}\left(w_{j}, x^{0}\right)+\mathcal{O}\left(\varepsilon^{-3 \theta} \boldsymbol{\delta}_{j}\right)\right] d x^{s}\right| \\
& \quad \leq C_{\#}\left(b \boldsymbol{\delta}_{j}^{1+\varpi} \varepsilon^{-2 \theta+1}+\varepsilon^{-3 \theta} \boldsymbol{\delta}_{j}\right) \boldsymbol{\delta}_{j} \leq C_{\#}\left(\frac{\varepsilon^{-2 \theta+1}}{d\left(W_{A}, W_{B}\right)^{1+\varpi} b^{\varpi}}+\frac{\varepsilon^{-3 \theta}}{d\left(W_{A}, W_{B}\right) b}\right) \boldsymbol{\delta}_{j} .
\end{aligned}
$$

We may be left with the integral over the interval $\left[w_{M}, c r^{\theta}\right]$ which is trivially bounded by $C_{\#} \varepsilon^{-2 \theta} \boldsymbol{\delta}_{M} \leq C_{\#}\left[\varepsilon^{2 \theta} b d\left(W_{A}, W_{B}\right)\right]^{-1}$. The statement follows since the manifolds we are considering have length at most $c \varepsilon^{\theta}$, hence $\sum_{j=0}^{M-1} \boldsymbol{\delta}_{j} \leq c \varepsilon^{\theta}$.

Proof of Lemma 8.9. We start by introducing a function $\bar{R}: W \rightarrow \mathbb{N}$ such that $\bar{R}(\xi)$ is the first $t \in \mathbb{N}$ at which $\Phi_{-t \tau_{-}} \xi$ belongs to a component of $\Phi_{-t \tau_{-}} W$ of size larger than $\kappa_{*} L_{0}, \kappa_{*}<1 / 3$, and distant more than $L_{0}$ from $\partial \Omega_{0}$.

Remark B.1. We choose $\kappa_{*}$ such that, if $\Phi_{-t \tau_{-}} W$ is a regular piece of size larger than $L_{0} / 3$ but in an $L_{0}$ neighborhood of $\partial \Omega_{0}$, then either $\Phi_{-(t+k) \tau_{-}} W$ or $\Phi_{-(t-k) \tau_{-}} W$ will satisfy our requirement for some $C_{\#}>k \tau_{-}>L_{0}$.

47 Note that $M$ exists and is finite by the first part of the lemma, 8.41). 
We define then $R(\xi)=\min \{\bar{R}(\xi), \ell\}$. Let $\mathcal{P}=\left\{J_{i}\right\}$ be the coarser partition of $W$ in intervals on which $R$ is constant. Note that, for each $W_{B, i}, \Phi_{\ell \tau_{-}}\left(W_{B, i}\right) \subset J_{j}$ for some $J_{j} \in \mathcal{P}$.

Let $\Sigma_{\ell, j}=\left\{(B, i): i \in \mathbb{N}, B \in E_{\ell, i}, d\left(W_{B, i}, W_{A, i}^{0}\right) \leq \rho_{*}, \Phi_{\ell \tau_{-}} W_{B, i} \subset J_{j}\right\}$. Then, by Lemma 3.5, for each $(B, i) \in \Sigma_{\ell, j}$

$$
Z_{\ell, B, i}=\int_{W_{B, i}} J_{\ell \tau_{-}}^{s} \leq C_{\#} \frac{\left|J_{j}\right|}{\left|\bar{W}_{j}\right|} \int_{W_{B, i}} J_{\left(\ell-R_{j}\right) \tau_{-}}^{s} \leq C_{\#} \frac{\left|J_{j}\right|}{\left|\bar{W}_{j}\right|}\left|\Phi_{\left(\ell-R_{j}\right) \tau_{-}} W_{B, i}\right|,
$$

where $R_{j}=R\left(J_{j}\right)$ and $\bar{W}_{j}=\Phi_{-R_{j} \tau_{-}} J_{j}$. Note that, by construction, either $\left|\bar{W}_{j}\right| \geq \kappa_{*} L_{0}$ or $R_{j}=\ell$ and $\bar{W}_{j}=W_{B, i}$. Next, consider the local weak stable surfaces $W_{B, i}^{0}:=\cup_{t \in\left[-c r^{\theta}, c r^{\theta}\right]} \Phi_{t} W_{B, i}$ and $\bar{W}_{j}^{0}:=\cup_{t \in\left[-c r^{\theta}, c r^{\theta}\right]} \Phi_{t} \bar{W}_{j}$.

Let us analyze first the case in which $R_{j}<\ell$. Let $\rho \leq C_{\#} L_{0}^{\frac{5}{3}}$. Then, by assumption, $\bar{W}_{j}$ is a manifold with satisfies condition (a) at the beginning of Section 6 . Indeed $\bar{W}_{j}$ is too long to belong to $\mathbb{H}_{k}$ with $k \geq C \rho^{-\frac{1}{5}}$, so that $\bar{W}_{j}$ satisfies condition (b) of that section as well. We can then use the construction in Section [6 to define an approximate unstable foliation in a $\rho$ neighborhood of the surface $\bar{W}_{j}^{0}$. Let $\Gamma_{B, i}$ be the set of leaves that intersect $\Delta_{\ell-R_{j}} \cap \Phi_{\left(\ell-R_{j}\right) \tau_{-}} W_{B, i}^{0}$. By the construction of the covering $B_{c \varepsilon} \theta\left(x_{i}\right)$, the $\Gamma_{B, i}$ can have at most $C_{\#}$ overlaps and since $W_{B}$ completely crosses $B_{c \varepsilon^{\theta}}\left(x_{i}\right)$, there can be no gaps between the curves in $\Gamma_{B, i}$. Now using the uniform transversality between the stable, unstable and flow directions,

$$
\sum_{(B, i) \in \Sigma_{\ell, j}} m\left(\Gamma_{B, i}\right) \geq C_{\#} \sum_{(B, i) \in \Sigma_{\ell, j}}\left|\Phi_{\left(\ell-R_{j}\right) \tau_{-}} W_{B, i}\right| \rho \varepsilon^{\theta} .
$$

Accordingly, for each $j$ such that $R_{j}<\ell$, remembering (B.10),

$$
\sum_{(B, i) \in \Sigma_{\ell, j}} Z_{\ell, B, i} \leq C_{\#}\left|J_{j}\right| \rho^{-1} \varepsilon^{-\theta} \sum_{(B, i) \in \Sigma_{\ell, j}} m\left(\Phi_{-\left(\ell-R_{j}\right) \tau_{-}} \Gamma_{B, i}\right),
$$

where we have used the invariance of the volume. Remembering that the $\Phi_{-\left(\ell-R_{j}\right) \tau_{-}} \Gamma_{B, i}$ have a fixed maximal number of overlaps and since they must be all contained in a box containing $\widetilde{O}$ of length $C_{\#} \varepsilon^{\theta}$ in the flow direction, of length $C_{\#} \varepsilon^{\theta}$ in the stable direction and of length $C_{\#}\left(\rho_{*}+\Lambda^{-\left(\ell-R_{j}\right) \tau_{-}} \rho\right)$ in the unstable direction, we have 48

$$
\sum_{j} \sum_{(B, i) \in \Sigma_{\ell, j}} Z_{B, i} \leq \sum_{\left\{j: R_{j} \leq \frac{\ell}{2}\right\}} C_{\#}\left|J_{j}\right| \rho^{-1} \varepsilon^{\theta}\left(\rho_{*}+\Lambda^{-\frac{\ell}{2} \tau_{-}} \rho\right)+\sum_{\left\{j: R_{j}>\frac{\ell}{2}\right\}} \sum_{(B, i) \in \Sigma_{\ell, j}} Z_{B, i} .
$$

To estimate the sum on $R_{j}>\frac{\ell}{2}$ we use the growth Lemma 3.8(a), with $1 / q_{0}=0$, which, remembering Remark B.1, implies

$$
\begin{aligned}
\sum_{\left\{j: R_{j}>\frac{\ell}{2}\right\}} \sum_{(B, i) \in \Sigma_{\ell, j}} Z_{B, i} & \leq \sum_{\left\{j: R_{j}>\frac{\ell}{2}\right\}} \sum_{(B, i) \in \Sigma_{\ell, j}} C_{\#} \int_{\Phi_{\ell \tau_{-} / 2} W_{B, i}} J_{\Phi_{\ell \tau_{-} / 2} W_{B, i}} \Phi_{\ell \tau_{-} / 2} \\
& \leq \sum_{W_{B} \in \mathcal{I}_{\ell \tau_{-} / 2}(W)} C_{\#} \int_{W_{B}} J_{W_{B}}^{s} \Phi_{\ell \tau_{-} / 2} \\
& \leq \sum_{W_{B} \in \mathcal{I}_{\ell \tau_{-} / 2}(W)} C_{\#} L_{0}\left|J_{W_{B}}^{s} \Phi_{\ell \tau_{-} / 2}\right|_{\mathcal{C}^{0}} \leq C_{\#} \lambda^{\ell \tau_{-} / 2} .
\end{aligned}
$$

Since $\lambda>\Lambda^{-1}$, the lemma follows by choosing $\rho=\rho_{*}^{1 / 2}$.

\footnotetext{
48 Remember that the $J_{j}$ are all disjoint and $\cup_{j} J_{j}=W$ and $|W| \leq L_{0}$.
} 


\section{REFERENCES}

[AM] V. Araújo and I. Melbourne, Exponential decay of correlations for nonuniformly hyperbolic flows with a $C^{1+\alpha}$ stable foliation, including the classical Lorenz attractor, Ann. Henri Poincaré 17 (2016) 2975-3004.

[AGY] A. Avila, S. Gouëzel, and J.-C. Yoccoz, Exponential mixing for the Teichmüller flow, Pub. Math. IHÉS 104 (2006) 143-211.

[Ar] V. Arnold, Mathematical Methods of Classical Mechanics, Graduate Texts in Math. 60, Springer-Verlag, Berlin-New York $(1978,1989)$.

[BG] V. Baladi and S. Gouëzel, Banach spaces for piecewise cone hyperbolic maps, J. Mod. Dyn. 4 (2010) 91-137.

[BL] V. Baladi and C. Liverani, Exponential decay of correlations for piecewise cone hyperbolic contact flows, Comm. Math. Phys. 314 (2012) 689-773.

[BT1] V. Baladi and M. Tsujii, Anisotropic Hölder and Sobolev spaces for hyperbolic diffeomorphisms, Annales de l'Institut Fourier 57 (2007) 127-154.

[BT2] V. Baladi and M. Tsujii, Dynamical determinants and spectrum for hyperbolic diffeomorphisms, pp. 29-68, in Probabilistic and Geometric Structures in Dynamics, Contemp. Math. 469 (Amer. Math. Soc.) (2008).

[BV] V. Baladi and B. Vallée, Exponential decay of correlations for surface semi-flows without finite Markov partitions, Proc. Amer. Math. Soc. 133 (2005) 865-874.

[BSC] L. A. Bunimovich, Ya. G. Sinai, and N. I. Chernov, Statistical properties of two-dimensional hyperbolic billiards, Uspekhi Mat. Nauk 46 (1991) 43-92; translation in Russian Math. Surveys 46 (1991) 47-106.

[BMMW] K. Burns, C. Matheus, H. Masur, and A. Wilkinson Rates of mixing for the Weil-Petersson geodesic flow: Exponential mixing in exceptional moduli spaces, arXiv:1605.09037, to appear GAFA.

[Bu] O. Butterley, A note on operator semigroups associated to chaotic flows, Ergodic Theory Dynam. Systems 36 (2016) 1396-1408. (Corrigendum: 36(2016) 1409-1410).

[BuL] O. Butterley and C. Liverani, Robustly invariant sets in fibre contracting bundle flows, J. Mod. Dyn., 7 (2013) 153-208.

[C1] N. Chernov, Decay of correlations in dispersing billiards, J. Stat. Phys. 94 (1999) 513-556.

[C2] N. Chernov, A stretched exponential bound on time correlations for billiard flows, J. Stat. Phys. 127 (2007) 21-50.

[CM] N. Chernov and R. Markarian, Chaotic Billiards, Mathematical Surveys and Monographs 127 (2006).

[Da] E.B. Davies, Linear Operators and their Spectra, Cambridge Studies in Advanced Mathematics 106 (2007).

[DL] M.F. Demers and C. Liverani, Stability of statistical properties in two-dimensional piecewise hyperbolic maps, Trans. American Math. Soc. 360 (2008) 4777-4814.

[DZ1] M.F. Demers and H.-K. Zhang, Spectral analysis for the transfer operator for the Lorentz gas, J. Mod. Dyn. 5 (2011) 665-709.

[DZ2] M.F. Demers and H.-K. Zhang, A functional analytic approach to perturbations of the Lorentz gas, Comm. Math. Phys. 324 (2013) 767-830.

[DZ3] M.F. Demers and H.-K. Zhang, Spectral analysis of hyperbolic systems with singularities, Nonlinearity 27 (2014) 379-433.

[Do] D. Dolgopyat, On decay of correlations in Anosov flows, Ann. of Math. 147 (1998) 357-390.

[EG] L.C. Evans and R.F. Gariepy, Measure theory and fine properties of functions, Studies in Advanced Mathematics, CRC Press, Boca Raton, FL, 1992.

[FRS] F. Faure, N. Roy, and J. Sjöstrand, Semi-classical approach for Anosov diffeomorphisms and Ruelle resonances, Open Math. J. 1 (2008) 35-81.

[FM] B. Friedman and R. F. Martin, Behavior of the velocity autocorrelation function for the periodic Lorentz gas, Phys. D 30 (1988) 219-227.

[GLP] P. Giulietti, C. Liverani, and M. Pollicott, Anosov flows and dynamical zeta functions, Ann. of Math. 178 (2013) 687-773.

[GL] S. Gouëzel and C. Liverani, Banach spaces adapted to Anosov systems, Ergodic Theory Dynam. Systems 26 (2006) 189-218.

[H] H. Hennion, Sur un théorème spectral et son application aux noyaux lipchitziens, Proc. Amer. Math. Soc. 188 (1993), 627-634.

[I] Y. Iwata, A generalized local limit theorem for mixing semi-flows, Hokkaido Math. J. 37 (2008) 215-240.

[Ka] T. Kato, Perturbation theory for linear operators. Reprint of the 1980 edition, Classics in Mathematics, Springer-Verlag, Berlin, 1995.

$[\mathrm{KH}] \quad$ A. Katok and B. Hasselblatt, Introduction to the modern theory of dynamical systems, Cambridge University Press, 1995. 
[KS] A. Katok, J.-M. Strelcyn, F. Ledrappier, and F. Przytycki, Invariant manifolds, entropy and billiards; smooth maps with singularities, Lecture Notes in Mathematics, 1222. Springer-Verlag, Berlin, 1986.

[LP] S. Le Borgne and F. Pène, Vitesse dans le théorème limite central pour certains systèmes dynamiques quasi-hyperboliques, Bull. Soc. Math. France 133 (2005) 395-417.

[L1] C. Liverani, Decay of correlations. Ann. of Math. 142 (1995) 239-301.

[L2] C. Liverani, On contact Anosov flows, Ann. of Math. 159 (2004) 1275-1312.

[MM] H. Matsuoka and R.F. Martin, Long-time tails of the velocity autocorrelation functions for the triangular periodic Lorentz gas, J. Stat. Phys. 88 (1997) 81-103.

[M] I. Melbourne, Rapid decay of correlations for nonuniformly hyperbolic flows, Trans. Amer. Math. Soc. 359 (2007) 2421-2441.

[MN] I. Melbourne and M. Nicol, Large deviations for nonuniformly hyperbolic systems, Trans. Amer. Math. Soc. 360 (2008) 6661-6676.

[MT] I. Melbourne and A. Török, Statistical limit theorems for suspension flows, Israel J. Math. 144 (2004) 191-209.

[Pe] F. Pène, A Berry Esseen result for the billiard transformation, Preprint 2007. <hal-01101281>

[R] D. Ruelle, A measure associated to Axiom A attractors, Amer. J. Math. 98 (1976) 619-654.

[RS] T. Runst and W. Sickel, Sobolev spaces of fractional order, Nemytskij operators, and nonlinear partial differential equations, Walter de Gruyter \& Co., Berlin, 1996.

[S1] Ya. G. Sinai, On the foundations of the ergodic hypothesis for a dynamical system of statistical mechanics, Dokl. Akad. Nauk SSSR 153 1261-1264 (Russian); translated as Soviet Math. Dokl. 4 (1963) 1818-1822.

[S2] Ya. G. Sinai, Dynamical systems with elastic reflections. Ergodic properties of dispersing billiards (Russian) Uspehi Mat. Nauk 251970 (152) 141-192.

[S3] Ya. G. Sinai, Gibbs measures in ergodic theory, Russ. Math. Surveys 166 (1972) 21-69.

[Ts] M. Tsujii, Quasi-compactness of transfer operators for contact Anosov flows, Nonlinearity 23 (2010) 14951545.

[Y] L.-S. Young, Statistical properties of dynamical systems with some hyperbolicity. Ann. of Math. (2) 147 (1998) 585-650.

Sorbonne Universités, UPMC Univ. Paris 6, CNRS, Institut de Mathématiques de Jussieu, (IMJ-PRG), 4, Place Jussieu, 75005 Paris, France

E-mail address: viviane.baladi@imj-prg.fr

Department of Mathematics, Fairfield University, Fairfield CT 06824, USA

E-mail address: mdemers@fairfield.edu

Dipartimento di Matematica Università degli Studi di Roma Tor Vergata, Italy

E-mail address: liverani@mat.uniroma2.it 


\title{
ERRATUM FOR: EXPONENTIAL DECAY OF CORRELATIONS FOR FINITE HORIZON SINAI BILLIARD FLOWS
}

\author{
VIVIANE BALADI, MARK F. DEMERS, AND CARLANGELO LIVERANI
}

We are grateful to Malo Jézéquel who incited us to clarify Lemma 3.2 and (W2). We thank Damien Thomine for pointing out the flaws in Lemma 7.5 and in the proof of Lemma 4.6, and for requiring necessary clarifications in the definition of the neutral norm. Fortunately, this only affects the paper very locally, and we explain here how to amend it.

$$
\text { 1. Lemma 3.2 And (W2) — Definitions (3.10)-(3.11) And (4.3) }
$$

Lemma 3.2 does not show that there exists $\kappa_{0}$ such that if the curvature $\kappa$ of $W$ is bounded by $\kappa_{0}$ then the curvature of the iterated curve is bounded by $\kappa_{0}$. (The reason being that if $\kappa$ tends to zero then the quantity $B_{\xi}^{2} / B^{6}$ does not go to zero.) The proof of Lemma 3.2 shows that $F_{1}=B_{\xi} / B^{3}$ (where $B=d \omega / d \xi$, and $B_{\xi}=d B / d \xi$ ) is bounded for all times. So condition (W2) selecting those stable curves belonging to $\mathcal{W}^{s}$ should be replaced by the requirement that $F_{1}<C_{1}$ for some $C_{1}$. (Since Lemma 3.2 gives $\kappa^{2}<1 / 4+F_{1}^{2}$, it follows that all iterated curves have curvature $\kappa<\kappa_{0}$ for some $\kappa_{0}$. However $\mathcal{W}^{s}$ does not necessarily contain all stable curves with $\kappa<\kappa_{0}$ for some $\kappa_{0}$.)

The definitions (3.10) and (3.11) of $\mathcal{B}^{0}$ and $\mathcal{B}_{\sim}^{0}$ involve functions $f$ which are only continuous. We emphasize that "the neutral norm of $f$ is finite" then means that the derivative of $f$ in the flow direction exists almost everywhere on each $W \in \mathcal{W}^{s}$, and its integral against $\psi$ with $|\psi|_{C^{\alpha}(W)} \leq 1$ is uniformly bounded. (For example, if $f$ is a Cantor function in the flow direction, its neutral norm vanishes, while if $f \in C^{0}\left(\Omega_{0}\right) \backslash C_{\sim}^{0}$ then there are no Dirac contributions in the derivative.) Similarly, when proving (4.3) in Proposition 4.10 for $f \in C^{0}\left(\Omega_{0}\right) \cap \mathcal{B}^{0}$ with $f \notin C_{\sim}^{0}$, there are no Dirac contributions.

\section{Neutral norm estimate in Lemma 4.6}

Lemma 4.6 states that, for each $f \in \mathcal{B}$, we have $\lim _{t \downarrow 0}\left\|\mathcal{L}_{t} f-f\right\|_{\mathcal{B}}=0$. The proof of the neutral norm estimate in this lemma for $f \in \mathcal{C}^{2}\left(\Omega_{0}\right) \cap \mathcal{C}_{\sim}^{0}$ should be corrected as follows:

Take $f \in \mathcal{C}_{\sim}^{0} \cap \mathcal{C}^{2}\left(\Omega_{0}\right)$. Then $\left\|\mathcal{L}_{t} f-f\right\|_{s} \leq C t|\nabla f \cdot \hat{\eta}|_{\mathcal{C}^{0}\left(\Omega_{0}\right)}$ and $\left\|\mathcal{L}_{t} f-f\right\|_{u} \leq C t|\nabla f \cdot \hat{\eta}|_{\mathcal{C}^{1}\left(\Omega_{0}\right)}$ hold, with the proofs as published, where $\hat{\eta}$ represents the flow direction. In particular (4.30) holds since $\mathcal{L}_{t} f$ is Lipschitz on $\Omega$ for all $t \geq 0$. (In the flow direction, distances are preserved so that the flow preserves Lipschitz smoothness in the flow direction, even across collisions.)

The estimate written for the neutral norm (proved using both (4.3) and (4.30)) holds if $W \in \mathcal{W}^{s}$ undergoes no collisions under $\Phi_{-s}$ for $0 \leq s \leq t$. Now fix $t<\tau_{\min }$. Then each $W \in \mathcal{W}^{s}$ can undergo at most one collision by time $-t$.

If $W \in \mathcal{W}^{s}$ undergoes a collision by time $-t$, then partition $W$ into at most three connected pieces, at most two of which will make no collision by time $-t$, and one connected curve $W^{\prime} \subset W$ which will undergo a collision in this time interval. On the two pieces which will not undergo a collision, we estimate $\mathcal{L}_{t} f-f$ as before.

To estimate $\mathcal{L}_{t} f-f$ on $W^{\prime}$, we cannot use (4.3) as for the collisionless pieces, because of derivatives of jump discontinuities. Instead, we first note that $\left|W^{\prime}\right| \leq C \sqrt{t}$ due to the strict

Date: February 2020. 
convexity of scatterers and the opposing convexity of $W$. Next, for $\psi \in \mathcal{C}^{\alpha}(W)$ with $|\psi|_{\mathcal{C}^{\alpha}(W)} \leq 1$, we estimate using (4.30),

$$
\begin{gathered}
\left|\int_{W^{\prime}} \partial_{r}\left(\left(\mathcal{L}_{t} f-f\right) \circ \Phi_{r}\right)\right| r_{r=0} \psi d m_{W}|\leq| \int_{W^{\prime}} \mathcal{L}_{t}(\nabla f \cdot \hat{\eta}) \psi d m_{W}|+| \int_{W^{\prime}} \nabla f \cdot \hat{\eta} \psi d m_{W} \mid \\
\leq\left|W^{\prime}\right|^{1 / q}\left\|\mathcal{L}_{t}(\nabla f \cdot \hat{\eta})\right\|_{s}+\left|W^{\prime}\right|^{1 / q}\|\nabla f \cdot \hat{\eta}\|_{s} \leq C t^{1 /(2 q)}|\nabla f \cdot \hat{\eta}|_{\mathcal{C}^{0}\left(\Omega_{0}\right)} .
\end{gathered}
$$

This, together with the previous bounds, implies that $\left\|\mathcal{L}_{t} f-f\right\|_{\mathcal{B}} \leq C t^{1 /(2 q)}|\nabla f \cdot \hat{\eta}|_{\mathcal{C}^{1}\left(\Omega_{0}\right)}$, which proves Lemma 4.6 for $f \in \mathcal{C}_{\sim}^{0} \cap \mathcal{C}^{2}\left(\Omega_{0}\right)$. The approximation argument for general $f \in \mathcal{B}$ follows as in the published proof of Lemma 4.6 .

\section{Lemma 7.5 (And Lemma 3.9) And Theorem 1.2}

The first inequality in the fourth line of the proof of Lemma 7.5 is wrong, so that the neutral estimate there is flawed. As a consequence, we must replace the statement $C^{1}\left(\Omega_{0}\right) \subset \mathcal{B}$ of Lemma 7.5 (and the corresponding inclusion in Lemma 3.9, also mentioned after Definition 2.12) by the weaker inclusion

$$
C^{1}\left(\Omega_{0}\right) \cap C^{0}(\Omega) \subset \mathcal{B} .
$$

The original Lemma 7.5 is used in the paragraph before (5.1) to deduce that the domain of $X$ contains $C^{2}\left(\Omega_{0}\right)$. This consequence of the original Lemma 7.5 must be replaced by the observation that if $f \in C^{2}\left(\Omega_{0}\right) \cap C^{0}(\Omega)$ (so that $\nabla f \in C^{1}\left(\Omega_{0}\right)$ ) is such that $(\nabla f) \cdot \hat{\eta}$ belongs to $C^{0}(\Omega)$, then the corrected version of Lemma 7.5 implies that $f$ is in the domain of $X$, i.e. $X f \in \mathcal{B}$.

This consequence of the original Lemma 7.5 is also used in the statement and proof of Theorem 1.2, which must be modified accordingly. In the statement of Theorem 1.2, in addition to requiring that $f \in C^{2}\left(\Omega_{0}\right) \cap C^{0}(\Omega)$, we add the requirement that $(\nabla f) \cdot \hat{\eta} \in C^{0}(\Omega)$. Then, in the proof of Theorem 1.2 in Section 9.1, we replace the claim that Lemma 7.5 implies that

$C^{2}\left(\Omega_{0}\right) \cap C^{0}(\Omega) \subset \operatorname{Dom}(X)$ by the same observation as above: if $f \in C^{2}\left(\Omega_{0}\right) \cap C^{0}(\Omega)$ and $(\nabla f) \cdot \hat{\eta} \in C^{0}(\Omega)$, then $f \in \operatorname{Dom}(X)$. The estimates then hold as written. 